\title{
FLOODS IN FLORIDA
}

\section{MAGNITUDE AND FREQUENCY}

\author{
By R.W. Pride noth \\ $59-98$
}

Prepared in cooperation with Florida State Road Department
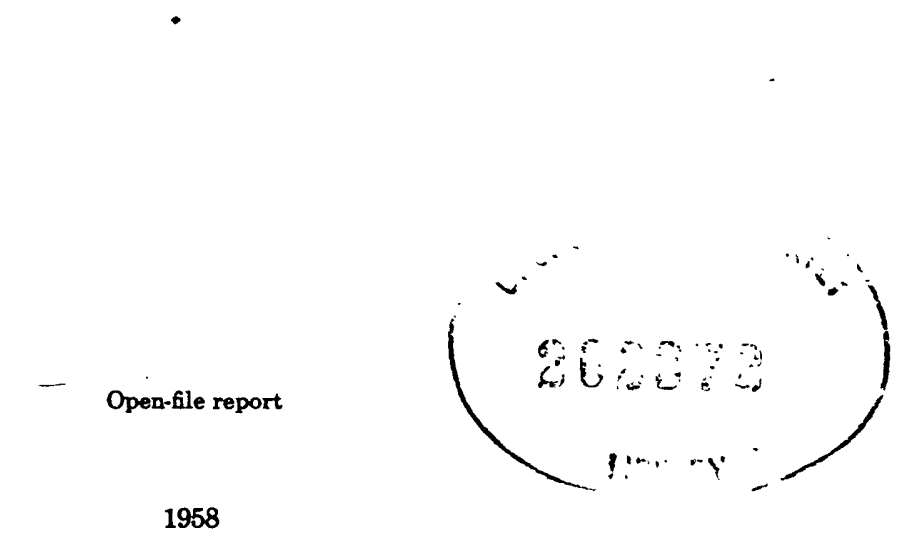

MAR $2 \quad$ YG57 


\section{CONTENTS}

Int roduction.

Acknowledgements

Description of the area

Topography

Coastal Lowlands

Central Highlands

Tallahassee Hills

Marianna Lowlands

Western Highlands.

Drainage basins.

St. Marys River

St. Johns River.

Lake Okechobee and the everglades.

Peace River

Withlacoochee River.

Suwannee River

Ochlockonee River.

A palachicola River Climate.

Flood records

Method of flood-frequency analysis

Flood frequency at a gaging station

The flood-frequency graph

Limitations of flood-frequency graphs . . . . . . . . . . . . . . . . . . . . 10

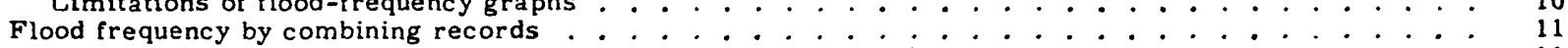

Comblning records by geometric similarity of frequency graphs. . . . . . . . . . . . . . . . . 11

Computation of mean annual floods ................................... 12

Test for geometric similarity ..... . . . . . . . . . . . . . . . . . . . . . . . . . . . . 12

Regional frequency graph . . . . . . . . . . . . . . . . . . . . . . 12

Combining records by similarity of mean annual floods
Factors influencing the mean annual flood.. .5
.

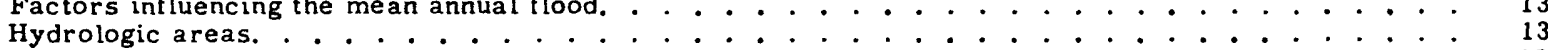

Flood frequency in Florida. .513

Flood regions of Florida. . . . . . . . . . . . . . . . . . . . . . . . . . . . . . 13

Delineation of flood regions. . . . . . . . . . . . . . . . . . . . . . . . . . . . 14

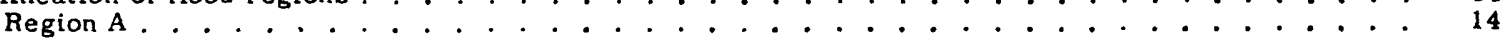

Region B . . . . . . . . . . . . . . . . . . . . . . . . . . . . . 14

Southern Florida

Hydrologic areas of Florida . . . . . . . . . . . . . . . . . . . . . . . . . . . . 16

Delineation of hydrologic areas . . . . . . . . . . . . . . . . . . . . 18

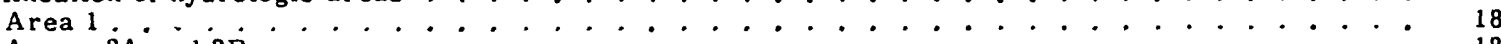

Areas $2 \mathrm{~A}$ and $2 \mathrm{~B}$

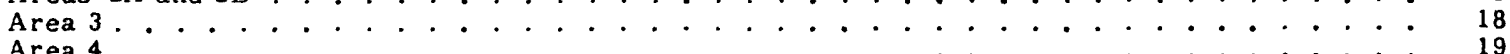



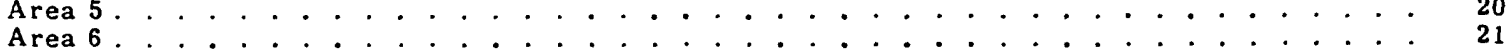

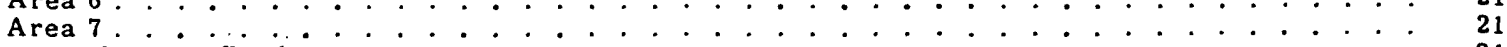

Frequency of annual flood stages . . . . . . . . . . . . . . . . . . . . . . . . . 21

St. Johns River . . . . . . . . . . . . . . . . . . . . . . . . . . . . . . . . . . . 22

Kissimmee River

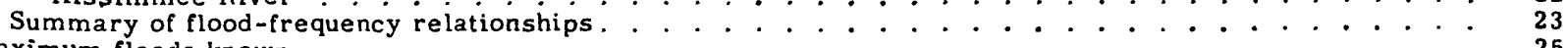

Maximum floods known. . . . . . . . . . . . . . . . . . . . . . . . . . . . . . . . . . . 25

Gaging station records.

Satilla River basin

Satilla River near Waycross, Ga. . . . . . . . . . . . . . . . . . . . . . . . . . . . . . . 38

Satilla River at Atkinson, Ga. . . . . . . . . . . . . . . . . . . . . . . . . . . . . . 38

St. Marys River basin. . . . . . . . . . . . . . . . . . . . . . . . . . . . . . . . . 39

North Prong St. Marys River at Moniac, Ga. . . . . . . . . . . . . . . . . . . . . . . . 39

St. Marys River near Macclenny, Fla . . . . . . . . . . . . . . . . . . . . . . . . . . . 40

St. Johns River basin. . . . . . . . . . . . . . . . . . . . . . . . . . . . . . 40

St. Johns River headwaters near vero Beach, Fla. . . . . . . . . . . . . . . . . . . . . . . . . 40

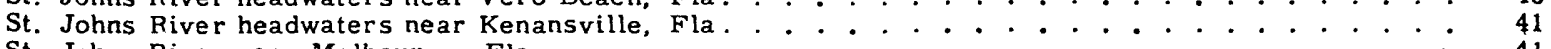

St. Johns River near Melbourne, Fla. . . . . . . . . . . . . . . . . . . . . . . . . . . . . . 41

Lake Washington near Eau Gallie, Fla . . . . . . . . . . . . . . . . . . . . . . . . . . . . . . 42

Lake Poinsett near Cocoa, Fla. . . . . . . . . . . . . . . . . . . . . . . 42

St. Johns River near Christmas, Fla . . . . . . . . . . . . . . . . . . . . . . . . . . . 43

Econlockhatchee River near Chuluota, Fla . . . . . . . . . . . . . . . . . . . . . . . . . . 44

St. Johns River above Lake Harney, near Geneva, Fla. . . . . . . . . . . . . . . . . . . . 44

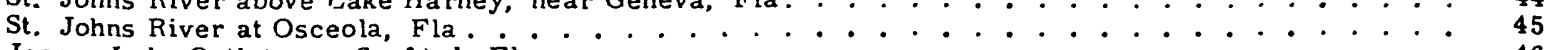

Jessup T,ake Outlet near Sanford, Fla . . . . . . . . . . . . . . . . . . . . . . . . . . . . . 46

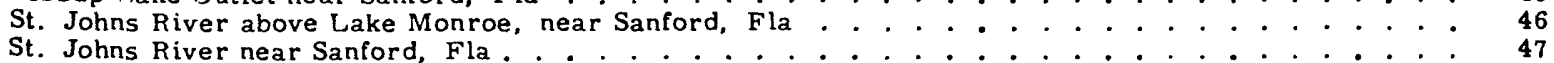


Gaging station records--Continued

St. Johns River basin--Continued

Wekiva River near Sanford, Fla.

St. Johns River near De Land, Fla
Lake A popka at Winter Garden, Fla

A popka-Beauclair Canal near Astatula, Fla.

Lake Dora at Mount Dora, Fla
Dead River near Tavares, Fla

Lake Minnehaha at Clermont, Fla.

Palatlakaha Creek near Mascotte, Fia.

Haines Creek at Lisbon. Fla

Lake Weir at Oklawaha, Fla

Oklawaha River at Moss Bluff, Fla

Oklawaha River near Ocala, Fla.

Silver Springs near Ocala, Fla

Oklawaha River near Conner, Fla .

Oklawaha River at Eureka, Fla.

Orange Lake near Boardman, Fla.

Orange Lake Outlet near Citra, Fla

Lochloosa Lake at Lochloosa, Fla.

Lochloosa Lake Outlet near Lochloosa, $\dot{\mathrm{F}}$

Orange Creek at Orange Springs, Fla

Oklawaha River near Orange Springs, Fla

Oklawaha River at Riverside Landing near Orange Springs, Fla

South Fork Black Creek near Penny Farms, Fla

North Fork Black Creek near Middleburg, Fla

Moultrie Creek basin.

Moultrie Creek near St. Augustine, Fla

Lake Okeechobee and the Everglades.

Lake Okeechobee, Fla

Fisheating Creek at Palmdale, Fla

Indian Prairie Canal near Okeechobee, Fla.

Alligator Lake near Ashton, Fla

Hart Lake near Narcoossee, Fla

East Tohopekaliga Lake at St. Cloud, Fla

Lake Tohopekaliga at Kissimmee, Fla.

Cypress Lake near St. Cloud, Fla.

Lake Butler at Windermere, Fla

Cypress Creek at Vineland, Fla.

Reedy Creek near Loughman, Fla.

Lake Hatchineha near Haines City, Fla

Lake Kissimmee near Lake Wales, Fla

Weohyakapka-Rosalie Canal nr. Lake Wales, Fla
Kissimmee River below Lake Kissimmee, Fla.

Kissimmee River at Fort Kissimmee, Fla

Reedy Lake Outlet near Frostproof, Fla.

Lake Arbuckle near Avon Park, Fla.

Josephine Creek near De Soto City, Fla

Lake Istokpoga near De Soto City, Fla.

Istokpoga Canal near Cornwell, Fla.

Kissimmee River near Cornwell, Fla.

Kissimmee River near Okeechobee, Fla

St. Lucie Canal at Lake Okeechobee, Fla
West Palm Beach Canal at Canal Point, Fla

West Palm Beach Canal at West Palm Beach, Fla,

Hillsboro Canal at Belle Glade, Fla.

Hillsboro Canal at Shawano Plantation, Fla

Hillsboro Canal near Deerfield Beach, Fla.

North New River Canal at South Bay, Fla

North New River Canal at 26 -mile bend, near Fort Lauderdale, $\dot{F}$ la

North New River Canal near Fort Lauderdale, Fla

Miami Canal at Pennsuco, near Miami, Fla

Tamiami Canal outlets, Miami to Monroe, Fia

Lake Trafford near Immokalee, Fla.

Imperial River near Bonita Springs, Fla

Caloosahatchee Canal at Moore Haven, Fla

Orange River near Fort Myers, Fla.

Peace River basin.

Drainage Canal west of Dundee, Fla.

Peace Creek Marsh outlet near Alturas, Fia

Lake Howard at Winter Haven, Fla

Lulu Lake Outlet at Eloise, Fla.

Peace River at Bartow, Fla -

Peace River at Arcadia, Fla. 
Gaging station records--Continued

Miakka River basin. . . . . . . . . . . . . . . . . . . . 99

Miakka River near Sarasota, Fla . . . . . . . . . . . . . . . . . . . . . . 99

Manatee River basin . . . . . . . . . . . . 99

Manatee River near Bradenton, Fla ................................99

Little Manatee River basin

Little Manatee River near Wimauma, fla . . . . . . . . . . . . . . . . . . . . 100

Alafia River basin Alafia River at Lithia, Fla . . . . . . . . . . . . . . . . . . . . . . . . 101

Hillsborough River basin. . . . . . . . . . . . . . 101

Hillsborough River near Zephyrhills, Fla . . ............ . . . . . . . . . .

Hillsborough River near Tampa, Fla. . . . . . . . . . . . . . . . . . . . . . . . . . 102

Lake Tarpon basin. . . . . . . . . . . . . . . . . . . . . 103 Brooker Creek near Odessa, Fla . . . . . . . . . . . . . . . . . . . . . . 103

Anclote River basin Anclote River near Elfers, Fla . . . . . . . . . . . . . . . . . . . . 103

Withlacoochee River basin

Withlacoochee River at Trilby, Fla . . . . . . . . 104

Withlacoochee River at Croom, Fla . . . . . . . . . . . . . . . . . . . . . . . 104

Withlacoochee River near Holder, Fla . . . . . . : . . . . . . . . . . . . . . 105

Waccasassa River basin . . . . . . . . . 106

Waccasassa River near Otter Creek, Fla . . . . . . . . . . . . . . . . . . . . . . . 106

Otter Creek at Otter Creek, Fla . . . . . . . . . . . . . . . . . . . 106

Suwannee River basin. . . . . . . . . . . . . . . . . . . . . . . . . . . . 107

Suwannee River at Fargo, Ga. . . . . . . . . . 107

Suwannee River at White Springs, Fla ............................. . . . . . . . . . . . . .

Alapaha River near Alapaha, Ga . . . . . . . . . . . . . . . . . . . . . . 108

Alapaha River at Statenville, Ga . . . . . . . 109

Little River near Adel, Ga . . . . . . . . . . . . . . . . . . . . . . . . . . . . 109

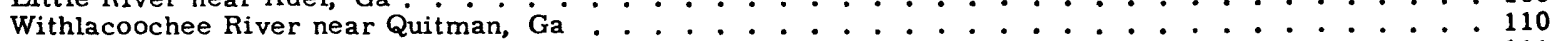

Withlacoochee River near Pinetta, Fla. . . . . . . . . . . . . . . . . . . . . . . 111

Suwannee River at Ellaville, Fla . . . . . . . . . . . . . . . . . . . . . . . . . . 111

Suwannee River at Luraville, Fla . . . . . . . . . . . . . . . . . . . . . . . 112

Suwannee River at Branford, Fla . . . . . . . . . . . . . . . . . . . . . . . 113

Santa Fe River at Worthington, Fla . . . . . . . . . . . . . . . . . . 113

Santa Fe River near High Springs, Fla. . . . . . . . . . . . . . . . . . . . . . . . . . 114

Santa Fe River near Fort White, Fla . . . . . . . . . . . . . . . . . . . . . . . 115

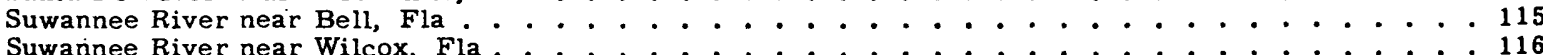

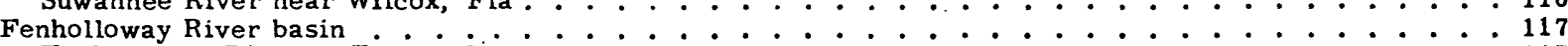

Fenholloway River at Foley, Fia

Ochlockonee River basin . . . . . . . . . . . . . . . . . 117

Ochlockonee River near Thomasville, Ga . . . . . . . . . . . . . . . . . . . . . . . 117

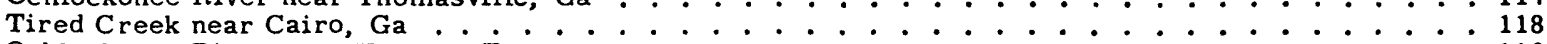

Ochlockonee River near Havana, Fla

Ochlockonee River near Bloxham, Fla

Apalachicola River basin . . . . . . . . . . . . . . . . . . . . . . . 120

Spring Creek near Iron City, Ga

Apalachicola River at Chattahoochee, fla

Mosquito Creek at Chattahoochee, Fla. . . . . . . . . . . . . . . . . 121

Apalachicola River near Blountstown, Fla . . . . . . . . . . . . . . . . . . . . . . . 122

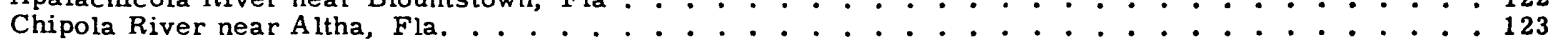

Bear Creek basin. . . . . . . . . . . . . . . . . . . . . .

Econfina Creek near Bennett, Fla

Choctawhatchee River basin . . . . . . . . . . . . . . . . . . . . . . . . . . . . . 124

Choctawhatchee River near Newton, Ala . . . . . . . . . . . . . . . . . . . . 124

Pea River near Samson, Ala . . . . . . . . . . . . . . . . . . . . . 125

Choctawhatchee River at Caryville, Fla . . . . . . . . . . . . . . . . . . . . 126

Choctawhatchee River near Bruce, Fla. . : . . . . . . . . . . . . . . . . . . . . 127

Yellow River basin .

Lightwood Knot Creek at Babbie, Ala

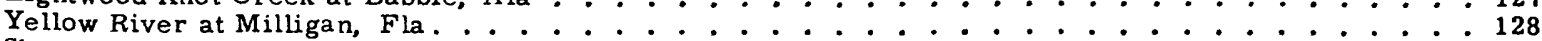

Shoal River near Crestview, Fla

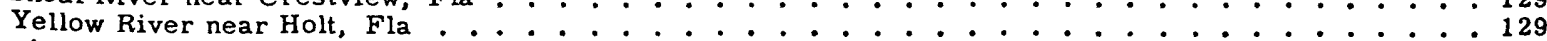

Blackwater River basin . . . . . . . . . . . . . . . . . . . . . . . . . . . 130

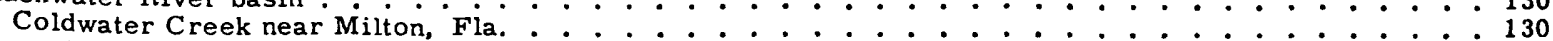

Escambia River basin. . . . . . . . . . . . . . . . . . . . . . . . . . . 131

Conecuh River near Andalusia, Ala . . . . . . . . . . . . . . . . . . . . . . . . . . 131

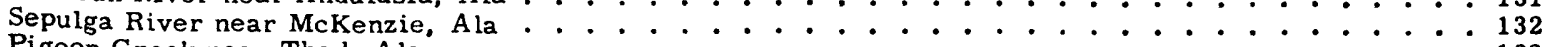

Pigeon Creek near Thad, Ala.

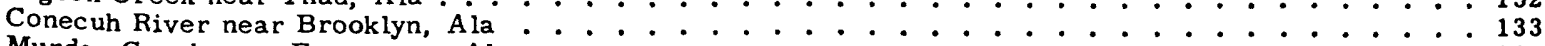

Murder Creek near Evergreen, Ala . . . . . . . . . . . . . . . . . . . . . . . . 134

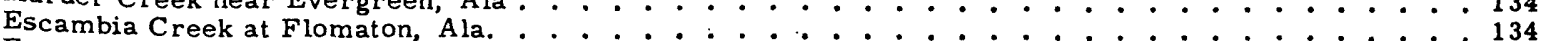

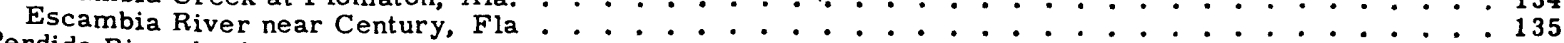

Perdido River basin. .

Perdido River at Barrineau Park, Fla. . . . . . . . . . . . . . . . . . . . . . . . . . . . 136 
Plate 1. Map showing hydrologic areas and flood regions of Florida. . . . . . . . . Inside back cover

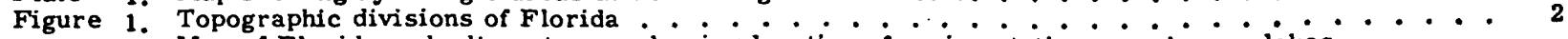

2. Map of Florida and adjacent area showing location of gaging stations on rivers, lakes, and canals . . . . . . . . . . . . . . . . . . . . . . 4 4

3. Average annual rainfall, in inches, in Florida . . . . . . . . . . . . . . . . 5

4. Maximum 24 -hour rainfall, in inches, to be expected once in 50 years....... .5

5. Homogeneity test graph . . . . . . . . . . . . . . . . . . 13

6. Homogeneous flood regions of Florida . . . . . . . . . . . . . . . . . . . . . 14

7. Composite frequency curve for floods in region $\mathrm{A} . . . . . . . . . . . . . . . . .15$

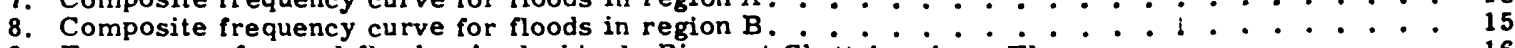

9. Frequency of annual floods, Apalachicola River at Chattahoochee, Fla ........... 16

10. Relation of lake area above gaging station to reduction of mean annual flood . . . . . . . . 17

11. Relation of mean annual flood to drainage area for area $1 . . . . . . . . . . . .18$

12. Relations of mean annual flood to drainage a rea along main stems of large rivers in Florida. : 19

13. Relation of mean annual flood to drainage area for areas $2 \mathrm{~A}, 2 \mathrm{~B}$, and $3 . \ldots . . . . . . . .20$

14. Relation of mean annual flood to drainage a rea for areas 4 and $5 . \ldots$

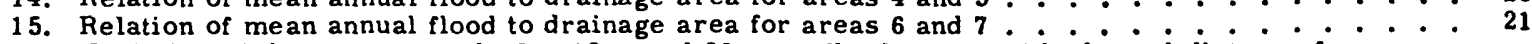

16. Variation of the mean annual, $5-, 10-$, and 30 -year flood stages with channel distance for main stem of St. Johns River, Florida . . . 22

17. Variation of the mean annual, $5-, 10-$, and 30 -year flood stages with channel distance for main stem of Kissimmee River, Florida. . . . . . . . . . . . . . . . . 23

18. Summary of relations of mean annual flood to drainage area for Florida. . . . . . . . . . . 24

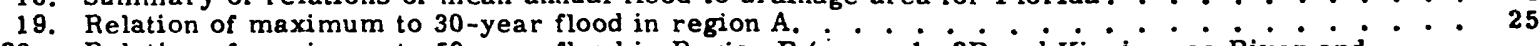

20a. Relation of maximum to 50 -year flood in Region $B$ (áreas $\dot{1}, 2 B$ and Kissimmee River and St. Johns River above Lake Harney). . . . . 26

20b. Relation of maximum to 50 -year flood in Region $B$ (areas 4,5 and Withlacoochee River and Suwannee River below Ellaville). . . . . . . . . . . . . . . . . . . . 27

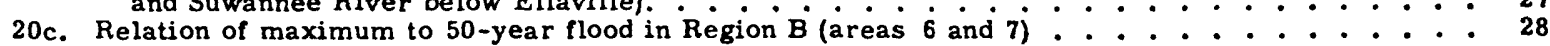

TABLES

Table 1. Gaging station data. . . . . . . . . . . . . . . . . . . . . . . 6

2. Adjustment of mean annual flood for attenuation by storage in lakes. . . . . . . . . . 17

3. Maximum known stages and discharges prior to 1956 in Florida . . . . . . . . . 29 


\title{
FLOODS IN FLORIDA
}

\section{MAGNITUDE AND FREQUENCY}

\author{
By R. W. Pride
}

\section{INTRODUCTION}

This report has been prepared to present a comprehensive summary of past floods in Florida and, from the pattern of recurrence of these floods, to develop an effective means of estimating the expectancy of floods in the future. Such information is a basic requirement in the proper design and location of structures placed in, across, or adjacent to flood plains.

When loss of life or extensive property damage would result if the structure is overtopped or destroyed, the design flood should be of such magnitude that it probably never will be exceeded. This report presents information on the maximum-known floods and rainfall in Florida that will be useful criteria for determining this ultimate design flood.

A much broader field of design, and one involving greater overall expenditures, concerns the selection of a design flood where loss of life is not a factor. Economic considerations dictate the basis for design in this field of engineering. Without knowledge of expectancy of floods, a structure may be so underdesigned that it suffers frequent damage or even destruction, or it may be so overdesigned that it will be damaged only by floods with recurrence intervals far greater than its probable'useful life. With a knowledge of flood expectancy, the design flood may be selected on the sound economic basis of calculated risk.

Ideally, there would be available at the site of each proposed structure a long-term systematic record of flood events. Unfortunately, this ideal situation seldom exists because it is obviously impracticable to maintain stream-gaging stations at all points where flood data might be desired. Even when the importance of the proposed structure would warrant the installation of a gaging station at the site, it is rarely possible to anticipate the need far enough in advance to obtain records of sufficient length. There is thus a need, not only for a method of relating flood magnitudes and frequencies at points where flood data are available, but for a method of transferring those data and relations to other points. This report represents an attempt to meet these needs in the State of Florida.

\section{Acknowledgements}

The data in this report were compiled and prepared for publication as part of a cooperative program for water-resources investigations between the U. S. Geological Survey and the Florida State Road Department. This program has been continuous since 1930 .

The report was prepared by R. W. Pride in the district office of the U. S. Geological Survey, Ocala, Fla., under the direction of $A$. O. Patterson, district engineer. Tate Dalrymple and others of the staff of the Washington office of the U. S. Geological Survey rendered valuable technical assistance in review of report and preparing text for publication.

Most of the data on flood peaks are from the original records in the files of the U. S. Geological Survey but some are from the records of the Corps of Engineers, U. S. Army, the U. S. Weather Bureau, the Everglades Drainage District, and the Okeechobee Flood Control District.

\section{Description of the Area}

When the old Spanish province of Florida was acquired by Great Britain in 1763, it was divided into two colonies, East Florida and West Florida, separated by the Apalachicola River. These geographic divisions now have no legal standing, but the name of West Florida is still in common use. East Florida, at least the southern part of it, is more commonly known as the Peninsula. The present State includes both East and West Florida.

Florida is the second largest State east of the Mississippi River, comprising an area of 58,666 square miles, of which 3,805 square miles are water. The State has 3,751 miles of tidal shore line, including the Atlantic and Gulf of Mexico coasts, and of these total lengths 1,987 miles outline the mainland and 1,764 miles surround islands. $1 /$

$1 /$ Douglas, E. M., Boundaries, areas, geographic centers, and altitudes of the United States and the Several States: U. S. Geol. Survey Bull. $817,1930$. 


\section{Topography}

The State of Florida is a part of a much larger geographic unit, the Floridan Plateau, that lies above sea level. The State is divisible into five natural topographic regions: Coastal Lowlands, Central Highlands, Tallahassee Hills, Marianna Lowlands, and Western Highlands. The general outlines of these regions are shown in figure 1. 2 J



Figure 1, - -Topogı aphic divisions of Florida.

Coastal Lowlands. - The Coastal Lowlands border the entire coast of Florida. They are widest in the south, where they cover all of the State south of Lake Okeechobee, and are narrowest from Choctawhatchee Bay to the west, where they are only 10 or 12 miles wide at Pensacola. Land elevation of the Coastal Lowlands varies from sea level to generally less than 100 feet above sea level.

Central Highlands. - - The Central Highlands extend from a 60-mile width along the Georgia line south-southeastward along the peninsula to the vicinity of Glades County, a distance of approximately 250 miles. This area includes high swampy plains, hills, and thousands of lakes. The altitude ranges from less than 40 feet above sea level in some valleys to 325 feet on the summit of Iron Mountain near Lake Wales.

Tallahassee Hills. - The Tallahassee Hills lie between the Georgia state line on the north and the Coastal Lowlands on the south--a width of nearly 25 miles--and between the Withlacoochee River on the east and the A palachicola River on the west--a length of 100 miles. Long gentle slopes with rounded summits are characteristic of the Tallahassee Hills except in the western part of Gadsden County where the upland surface consists of a nearly level plain which is about 300 feet above sea level.

Marianna Lowlands. -- The Marianna Lowlands, consisting of a rough quadrilateral area in Jackson, Holmes, and Washington Counties, is a region of low rolling hills and hollows. It is dotted with many sinks, ponds, and small lakes.

2/ Cooke, c. Wythe, Scenery of Florida: Florida Geological Survey Bull. No. 17. 1939. 
Western Highlands. - The Western Highlands extend from the Apalachicola River westward to the Perdido River. In the eastern part of this area they are confined between wide strips of Marianna Lowlands on the north and Coastal Lowlands on the south. Farther west, however, they extend from the Alabama line almost to the Gulf, from which they/are separated by a narrow strip of lowlands. The Western Highlands consist of a plateau sloping gently southward. The northern part is hilly, ranging up to 345 feet in Walton County. The southern part is a broad, gently rolling upland ranging in altitude from about 100 feet to 220 feet above sea level and is deeply trenched by narrow steep-walled valleys, which cut down nearly to sea level.

\section{Drainage basins}

On the Florida Peninsula the Central Highlands form the divide between the drainage to the east into the Atlantic Ocean and that to the south and west into the Gulf of Mexico. In Northwest Florida most of the area is drained by river systems that originate in Georgia or Alabama and flow southward through Florida to the Gulf of Mexico.

Because of the long coastline and narrow width of the State, much of the drainage is by many small coastal streams. This is in contrast with the few larger river systems that form the drainage pattern of the interior states.

The major river or drainage basins totally or partly within the State of Florida are the St. Marys, St. Johns, Lake Okeechobee and the Everglades, Peace, Withlacoochee, Suwannee, Ochlockonee, Apalachicola, Choctawhatchee, Yellow, Blackwater, Escambia, and Perdido. These river systems in Florida are shown in figure 2.

St. Marys River. - The St. Marys River originates in the Okefenokee Swamp in southern Georgia and meanders in an erratic course to the Atlantic Ocean. This river forms the northeastern boundary of Florida and drains an area of about 1,500 square miles.

St. Johns River. - - The St. Johns River is the largest river with its drainage area wholly within the State. The total drainage area of the St. Johns River is about 8,000 square miles. From its headwaters in the marshy area west of Vero Beach, it flows northward for about 250 miles to Jacksonville, where it turns eastward and enters the Atlantic Ocean at Mayport. The river has a very flat gradient and the effect of ocean tides extends upstream more than 100 miles. In the upper reaches of the St. Johns River, the channel expands intermittently to form several large lakes. In the lower reaches of the river between Palatka and Jacksonville, the width ranges from nearly 1 mile to more than 3 miles. Below Jacksonville the width is generally less than one-half mile. The largest tributary of the St. Johns River is the Oklawaha River, which drains an area of about 2,100 square miles. The source of the Oklawaha River is in a group of large lakes in the center of the State.

Lake Okeechobee and the Everglades. --This system drains the entire area of southern Florida. The predominant feature of this drainage system is Lake Okeechobee, which is a large, shallow, roughly circular freshwater lake about 700 square miles in area. The principal inlets to Lake Okeechobee are the Kissimmee River and Fisheating Creek. The Kissimmee River flows southward and enters the north side of the lake. Fisheating $\mathrm{Creek}$ flows southward for over half of its length and eastward for the remainder and enters the lake on its west side. In its former natural state Lake Okeechobee had no well-defined outlet; its rising waters would spread over the vast Everglades area to the south, or spill slowly into the flat areas west of the lake and find their way to the Caloosahatchee River, or escape by seepage and overland flow to the Allapattah, Hungryland, and Loxahatchee Sloughs to the east. Under present conditions Lake Okeechobee is impounded by levees that block the water from escaping into the everglades; its present main outlets are the St. Lucie Canal to the east and the Caloosahatchee Canal to the west.

The Everglades is a grassy marsh with an average width of 40 miles extending from the southern shore of Lake Okeechobee for about 100 miles to the sea at the southern end of Florida. Under natural conditions the waters of Lake Okeechobee would rise over the southern rim of the lake, push through the dense growth which formed that rim, and then move slowly southward through the dense sawgrass marsh. This original condition of the Everglades has been greatly altered by the development of drainage and flood-control works. The West Palm Beach, Hillsboro, North New River, and Miami Canals, originating at Lake Okeechobee, cut through the Everglades in a southeastward direction and provide some degree of drainage. At the present time the Everglades area is being further improved and reclaimed by the construction of additional canals, levees, reservoir areas, and pumping stations by the Corps of Engineers and the Central and Southern Florida Flood Control District. The generally low elevation and flat topography of southern Florida with its poor natural drainage results in slow runoff of excess water.

Peace River. --The Peace River originates in the lake region in Polk County and follows a southwestward course into Charlotte Harbor and the Gulf of Mexico. This river drains an area of about 2,000 square miles.

Withlacoochee River. - -The Withlacoochee River rises in a swampy area in Polk County and follows a northwestward course past Tsala Apopka Lake to the Gulf.

Suwannee River. --The Suwannee River heads in the Okefenokee Swamp in southern Georgia, flows southward over rapids nearly to White Springs, Florida, turns northwestward to its junction with the Alapaha River, thence southwestward past Dowling Park, then southeastward to the mouth of the Santa Fe River, and finally southward to the Gulf of Mexico. The principal tributaries of the Suwannee River are the Alapaha and Withlacoochee Rivers, which likewise originate in Georgia, and the Santa Fe River, which is entirely within the State of Florida. The total area drained by the Suwannee River basin is slightly less than 10,000 square miles. 


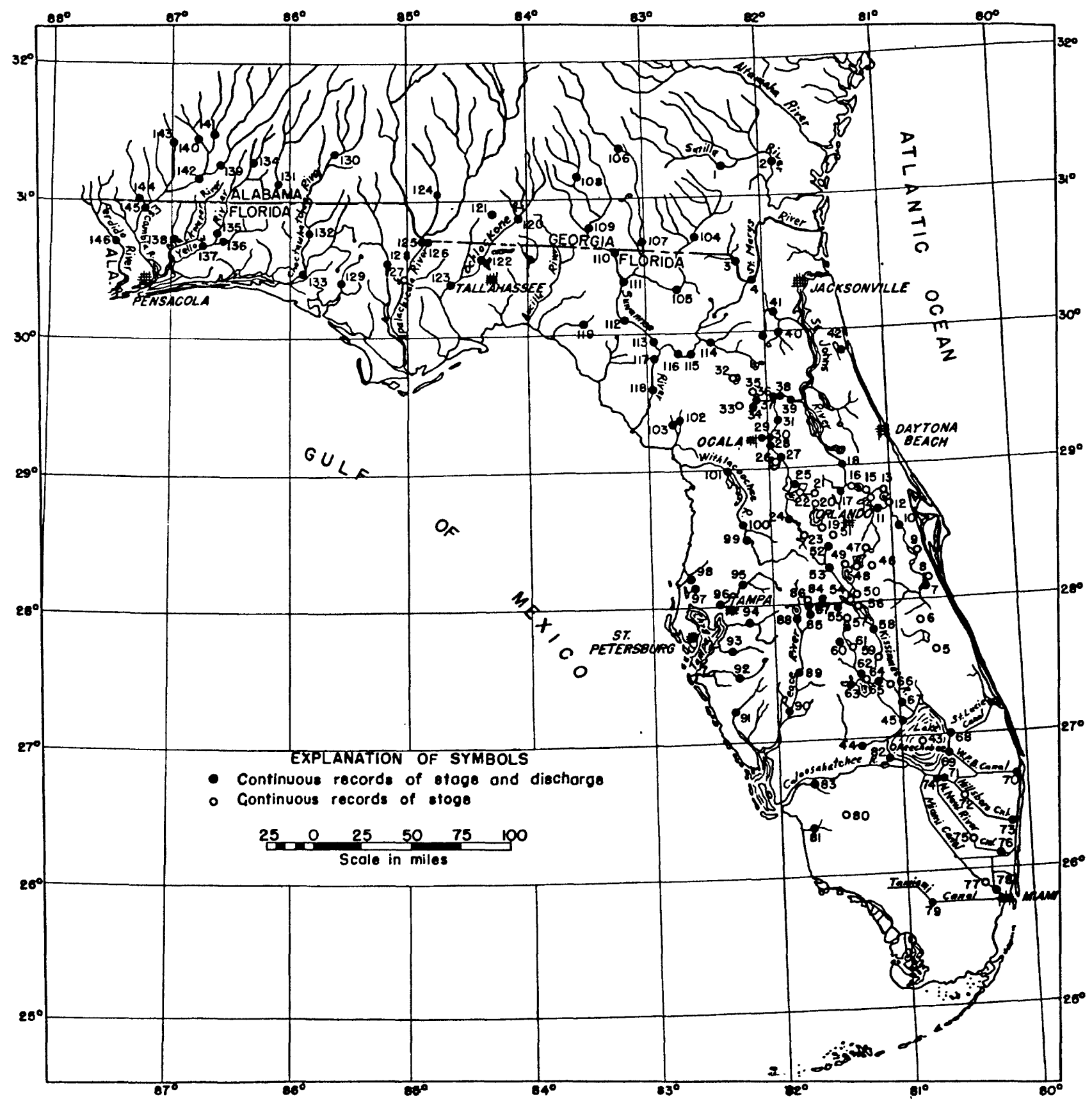

Figure 2. --Map of Florida and adjacent area showing location of gaging stations on rivers, lakes, and canals. 
Ochlockonee River. - The Ochlockonee River rises in Georgia and flows southward to the Gulf. The upper reach of this river has a fairly steep gradient. One of the few hydroelectric plants in Florida is located on this stream at the Jackson Bluff Dam west of Tallahassee.

Apalachicola River. -- The Apalachicola River is formed by the confluence of the Flint and the Chattahoochee Rivers at the Georgia-Florida State line. The drainage area at this point is 17,100 square miles and the river is by far the largest passing through the State. However, most of the total drainage area is in Georgia and Alabama and a relatively small area is in Florida. The largest tributary of the Apalachicola River in Florida is the Chipola River which rises in Alabama and flows southward in Florida to empty into the Apalachicola River just above its mouth at the Gulf.

Choctawhatchee, Yellow, Blackwater, Escambia, and Perdido Rivers. --All the larger rivers draining West Florida rise in southern Alabama and flow southward through Florida to the Gulf. These streams have fairly steep slopes and broad flood plains. The largest of these rivers and the total drainage area of each are as follows: Choctawhatchee, 4,646 square miles; Yellow, 1,365 square miles; Blackwater, 860 square miles; Escambia, 4,233 square miles; and Perdido, 925 square miles.

\section{Climate}

With its northernmost boundary at latitude $31^{\circ}$, the State of Florida is located well south in the temperate zone. Except on the north, the State is surrounded by waters of the Atlantic Ocean and Gulf of Mexico. No point in the State is more than 60 miles from salt water. This proximity to the sea, and the numerous inland bodies of water contribute to the causes that produce a temperate and a moist subhumid to humid climate. The slight variation in the altitude of the land surface has little effect on the overall climate of the State. With average temperatures of $81^{\circ} \mathrm{F}$. in July and $59^{\circ} \mathrm{F}$. in January, the climate is usually neither excessively hot nor excessively cold.

In the extreme southeastern and northwestern sections of the State the average annual rainfall is 64 inches, and at other places it is generally more than $\mathbf{4 8}$ inches except for the Florida Keys where rainfall totals are smaller. The yearly average over the State as a whole is $\mathbf{5 3}$ inches. The distribution of the average annual rainfall over the State is shown in figure 3.

There are well-defined wet and dry seasons in Florida. In West Florida and in the northern part of the Florida Peninsula, the period of greatest rainfall and consequent flooding usually occurs between November and April and is caused by broad cyclonic disturbances which commonly bring steady downpours over large areas of several southeastern States.

In central and southern Florida the wet season is usually from June through October. It is during this season that the tropical hurricanes, which originate in the doldrums, move in from the Caribbean Sea or the Atlantic Ocean. Although the winds of a hurricane rotate around a calm center at high velocities, sometimes 100 to 150 miles an hour, the progressive movement of the storm itself averages only about 12 miles an hour. $3 /$ These storms are usually accompanied by intense rainfall. Even though most hurricanes do not strike the Florida mainland, the coastal areas frequently receive heavy rains from many that move in a path fairly near the coast. These tropical storms gradually lose their intensity as they travel inland. This variation is illustrated by figure 4, which shows the 24-hour rainfall to be expected once in $\mathbf{5 0}$ years.

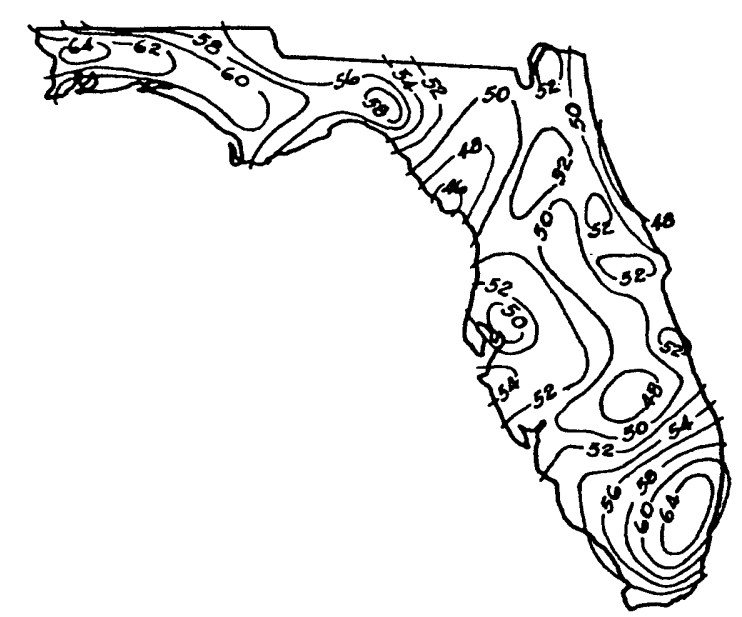

Figure 3. --Average annual rainfall, in inches, in Florida.

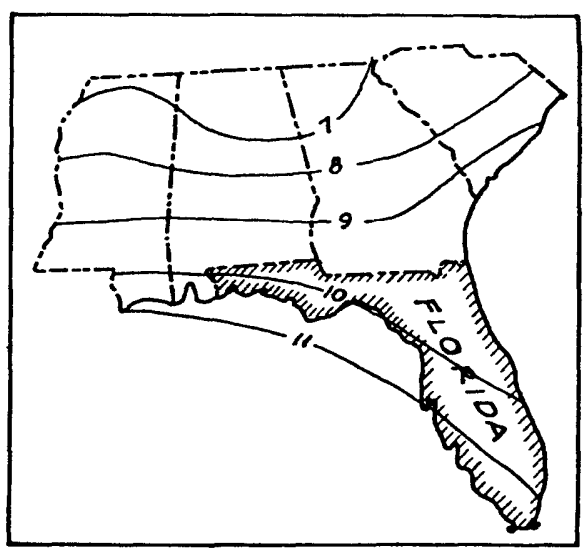

Figure 4. --Maximum 24-hour rainfall, in inches, to be expected once in 50 years. 
The base data for this report were obtained from records of gage height and discharge that have been collected by the Geological Survey and other agencies. All the larger streams in northern Florida head in Georgia or Alabama and records at upstream stations in these States have also been included.

The length of record of streamflow data in Florida is relatively short. In central and southern Florida very little systematic data had been collected before 1930. In northern Florida, records are available from a few gaging stations that were established as early as 1927. In this part of the State there are also records of stage at three stations operated by the U. S. Weather Bureau for a few years antedating Geological Survey record. Historical data pertaining to the peak stage of floods before the beginning of stream-gaging records have been included when such information could be obtained from reliable sources. Estimates of the peak discharge of these floods were made if there were reasonable assurance that the stage-discharge relation was applicable. The listing of the annual peaks in this report is by calendar year. This period was used because it more nearly coincides with the climatic year in Florida than does the water year beginning October 1.

Practically all of the discharge records presented in this report have been previously published in the annual Water-Supply Papers of the Geological Survey. Because of the importance of stage records and the inadequacy of discharge records, especially in southern Florida, there have also been included in this report considerable data on stage of lakes, as well as streams, from the unpublished files of the Ocala District office.

Records from several recently established stations or those for short periods during earlier years were not sufficiently long to be included for frequency analysis purposes. In general, flood records for all stations operated for 5 years or more are included in this study.

Gaging stations providing the base data are listed in downstream order in table 1, which shows by bar graph the years for which annual peak stages or discharges are available at each station. The location of each station is indicated by index number on the map of figure 2 .

Table 1.--Gaging station data

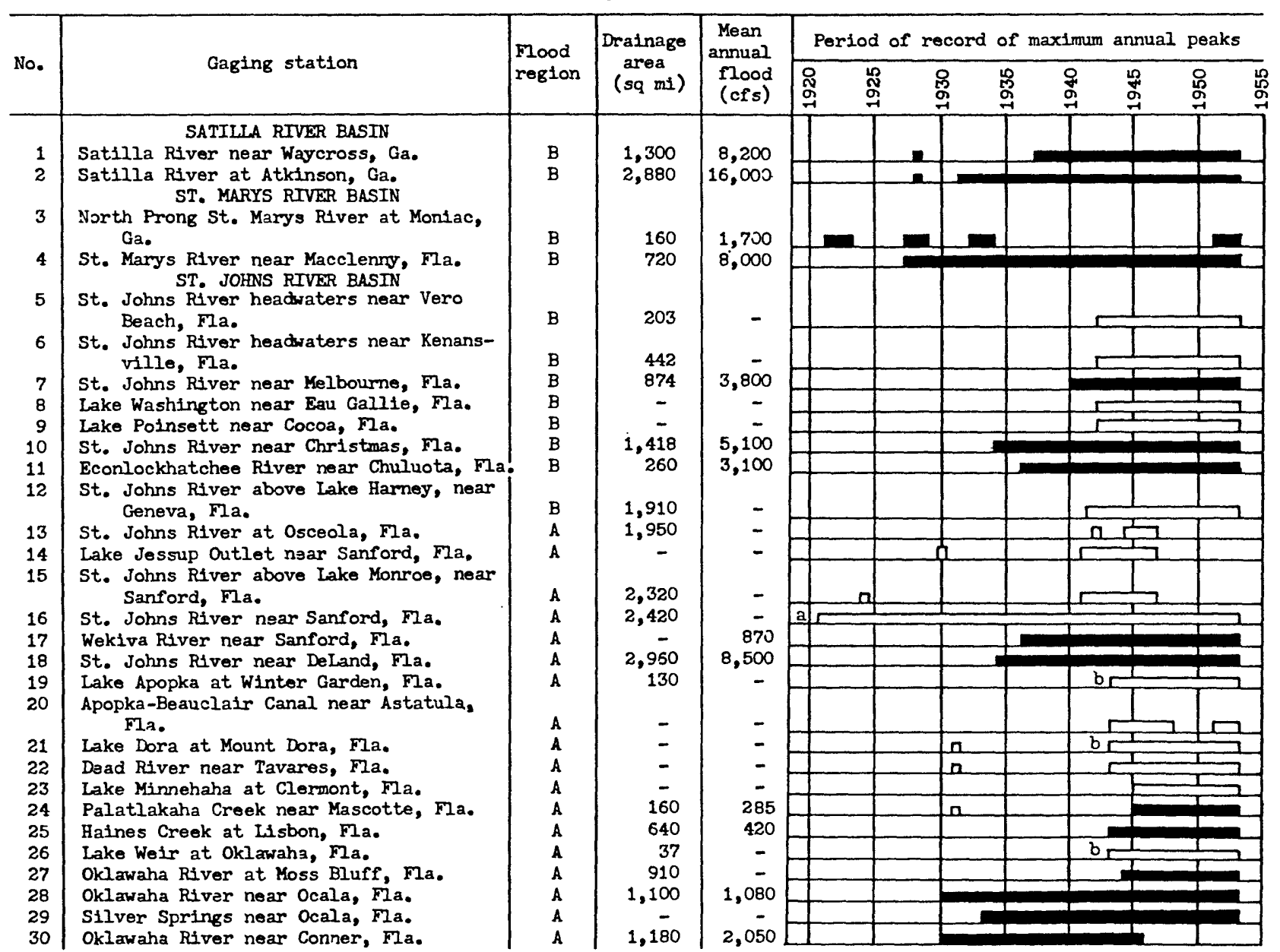


INTRODUCTION

Table 1.--Gaging station data--Contimied




FLOODS IN FLORIDA

Table 1.--Gaging station data--Continued

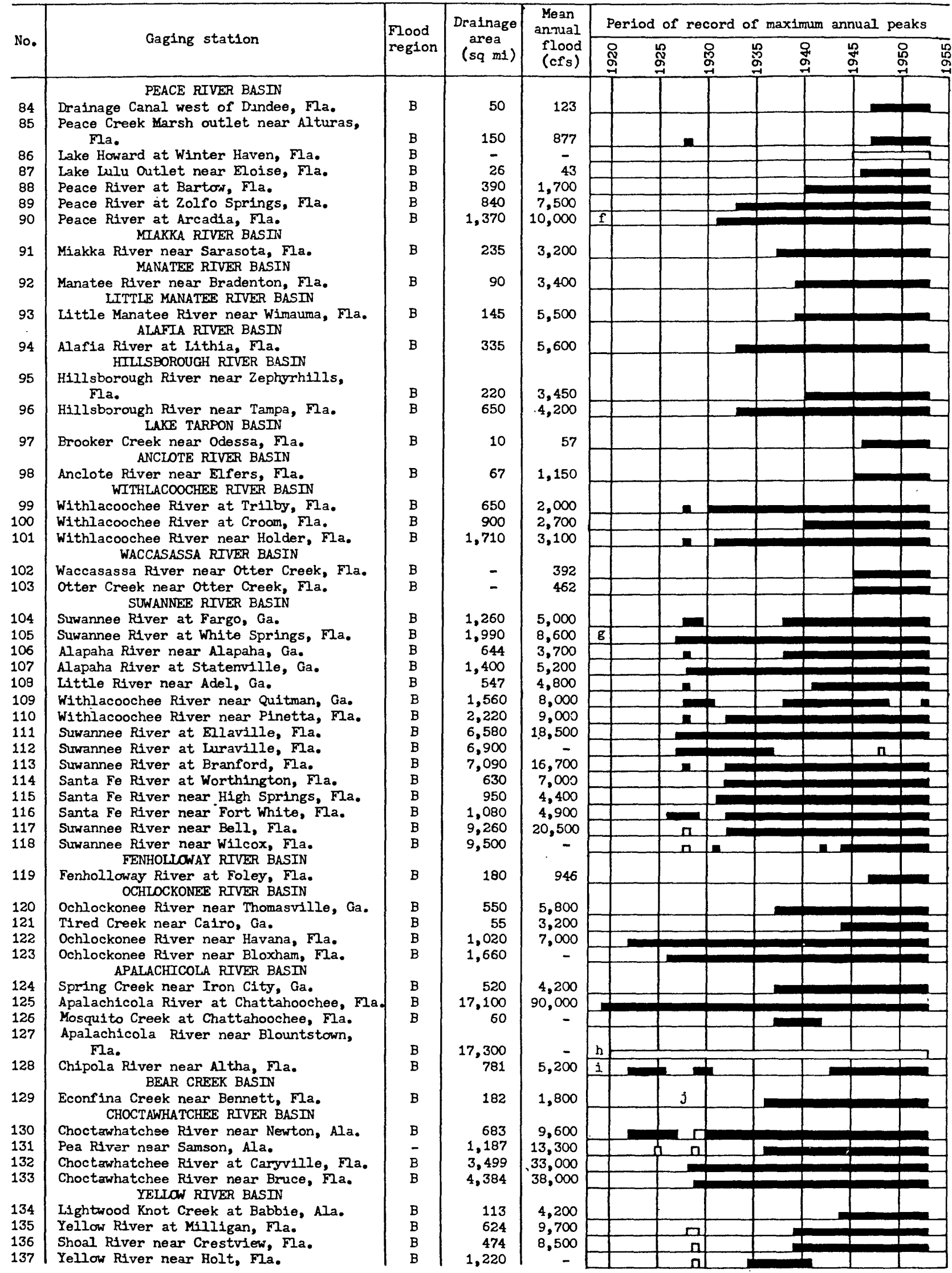


Table 1.--Gaging station data--Contimued

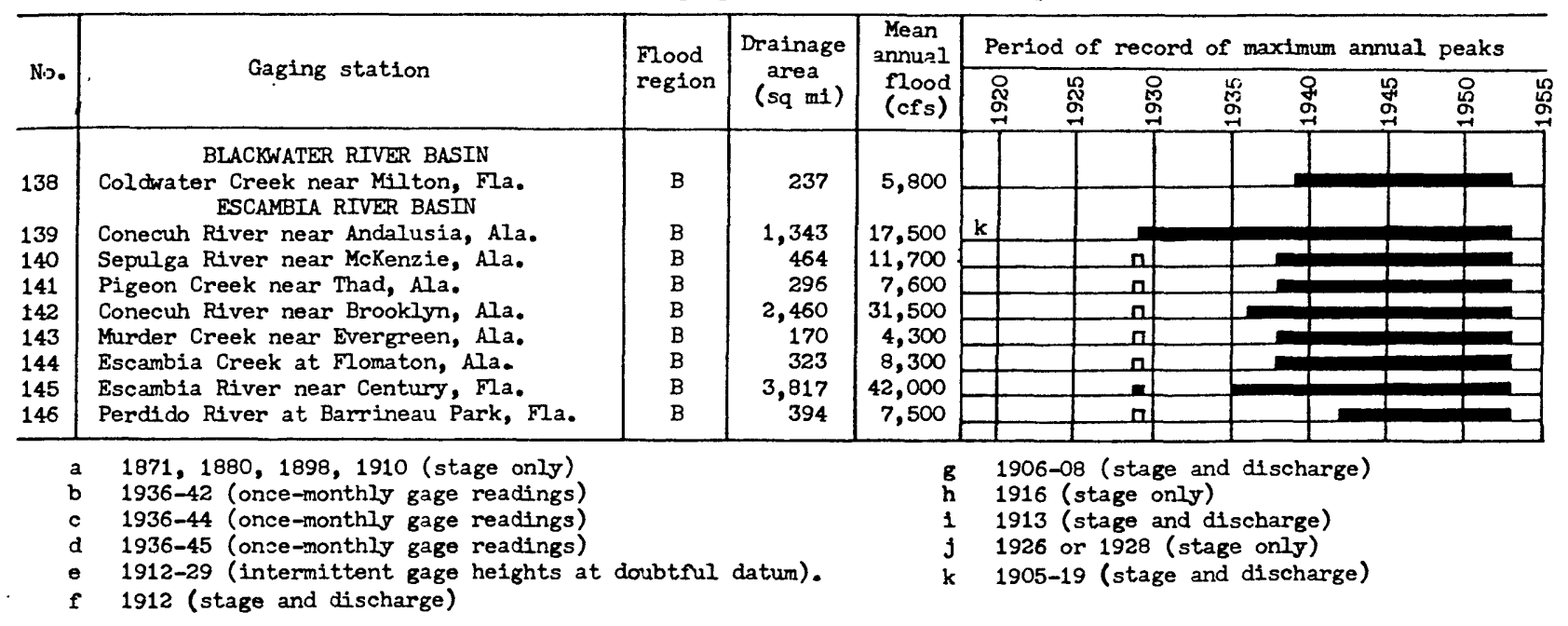

Bar symbols

Peak stage and discharge

Peak stage only

\section{METHOD OF FLOOD-FREQUENCY ANALYSIS}

The methods of computing flood frequency used in this report represent current techniques that have been developed in a continuing study by engineers of the Water Resources Division of the Geological Survey and by others. These methods serve, first, to define flood-frequency relations for a particular stream at a specific point of observation (at a gaging station); and, second, by combining a number of these point relations on an areal basis, to estimate flood-frequency relations at any point on any stream, gaged or ungaged, within a broad area (regional flood frequency).

\section{Flood frequency at a gaging station}

Flood data for a gaging station may be analyzed in two ways: as an annual flood series or as a partialduration series.

As used in this report, an annual flood is defined as the highest momentary peak discharge in a calendar year. In an annual flood series, only one flood, the annual maximum, is considered for each year of record. This method of analysis is sometimes objectionable because the second highest flood in a given year, which the above rule omits, may outrank many annual floods. This objection is overcome by the partial-duration series, in which all floods above a selected magnitude are considered without regard to the number within any given period. An objection to the partial-duration series is that not all of the floods considered may be fully independent events, that is, one flood may set the stage for another.

There is an important distinction in meaning between recurrence intervals determined by the two methods. In the annual-flood series the recurrence interval is the average interval in which a flood equal to or greater than a given magnitude will occur as an annual maximum. For example, a flood having a recurrence interval of 25 years, or the "25-year flood", is the flood that can be expected to be equal or exceeded four times in 100 years. No regularity of occurrence is implied; two 25 -year floods could occur in consecutive years. In the partial-duration series the recurrence interval is the average interval between floods of a given magnitude regardless of their relationship to the year or any other period of time. This distinction remains, even though for large floods the reccurrence intervals are practically the same on both scales.

From statistical principles, there is a definite relationship between the values in the two series. The following table 5 / shows comparative values of recurrence intervals for the two methods:

4 The general discussion of flood-frequency methods given herein was taken, in part, from similar reports for Georgia and A labama, published as Geological Survey Circulars 100 and 342, respectively. Dec. $194 \frac{5}{9}$.

5/ Langbein, W. B., Annual flood and the partial-duration series: Trans. Am. Geophys. Union, 
Annual flood series

\subsection{6}

1. 58

2. 00

2. 54

5. 52

10.5

20.5

50.5

100.5
Partial-duration series

0.5
1.0
1.45
2.0
5.0
10
20
50
00

This table shows that for recurrence intervals of 10 years and longer, or those commonly used for design purposes, both methods give essentially the same result. Because of its relative simplicity, the annual flood series has been used in this report.

\section{The tlood-frequency graph}

The annual floods for each complete year of record are listed in chronological order and numbered in descending order of magnitude--that is, beginning with the greatest as 1 . The next step is to fit a time scale to the data. Published methods for this operation are quite diverse, largely because of differing ideas as to the proper method of treating small samples. In the method adopted by the Geological Survey, recurrence intervals are computed as $(n+1) / m$, where $\underline{n}$ is the number of years of record and $\underline{m}$ is the relative order of magnitude of each $f$ lood. This formula is simple to compute. It is applicable both to the annual flood data and to the partial-duration series, and gives acceptable results conforming with some of the latest theories.

Annual floods are then plotted with discharges as ordinates and recurrence intervals as abscissae on a special coordinate paper 6 / designed to make the plotted points approximate a straight line.

After the annual floods have been plotted, it is necessary to fit a curve to the data. Several methods of fitting the curves analytically have been proposed, but curves so fitted, even by the most elaborate and painstaking methods, appear to offer no advantages over curves simply fitted by eye. Thus, for this report, the frequency curves were drawn as the line of best fit as determined visually, with consideration for the limitations and peculiarities inherent in the data. In general, the plotting positions of the extreme values were discounted or ignored in drawing the curves because there is little opportunity for determining the true recurrence interval of those values. (For an example of a typical frequency graph, refer to figure 9, page 16).

\section{Limitations of flood-frequency graphs}

Many hydraulic design problems currently being encountered in Florida require the determination of a design flood for a recurrence interval considerably longer than the period of record. As few flood records for the State extend for more than 25 years, most frequency curves must be extrapolated if the necessary information is to be obtained. For example, suppose that a flood record of 30 years is available for determining a required 50 -year flood. Generally, the computed plotting position of the greatest flood is not entirely dependable. Thus, the graph defining the 50-year flood might require extrapolation from the plotting position of the flood second (or possibly lower) in order of magnitude, that is, an extrapolation from about 15 to 50 years on the scale of recurrence intervals. Although this linear distance appears short on the graph, the error of the curve at its outer end could be considerable, regardless of the method of curve fitting or type of plotting paper used.

Another and more serious limitation of flood-frequency graphs based on relatively short records arises from the random manner in which flood events are distributed with respect to time. For example, a flood record of 100 years cannot be expected to include exactly one 100-year flood, two 50-year floods, three 33.3-year floods, and so on. If the 100-year record is separated into two 50-year periods, one period might include several 50 -year floods; the other none. Frequency graphs based on each of the two 50 -year periods may be vastly different and neither may closely resemble the frequency graph derived from the 100-year record considered as a whole. Similarly, the frequency graph obtained from the 100-year record could be appreciably different from that for a different period of the same length, or for a longer period. Thus, the record of annual floods for a particular stream is a random sample which may yield a frequency graph far different from one which would be derived from a record of infinite length.

The maximum departure to be expected between flood magnitudes or frequencies computed from relatively short records and their true (long-term) values decreases with the magnitude of the flood and with the length of the record. The variation, owing to the chance factor alone, between the flood magnitudes computed from records of varying length and the long-term values has been studied by Benson, ?/ who analyzed an array of 1,000 hypothetical

$6 /$ Powell, R. W., A Simple Method of Estimating Flood Frequency: Civil Eng. Feb. 1943.

7/ Benson, M. A., Characteristics of frequency curves based on a theoretical 1,000-year record: U. S. Geōlogical Survey, open-file report; 1952. 
annual floods distributed according to the theory of extreme values. ${ }^{8}$ / In using statistical methods, the most favorable expectancy during 95 percent of the time, or 19 chances out of $\overline{2} 0$, is commonly used as the criterion for dependable results. The following table based on Benson's study, shows the length of record necessary to define floods of selected frequency within 10 percent and 25 percent of the correct long-term $(1,000$-year $)$ value.

Length of record in years required to define flood within indicated percentage of correct value, 19 times in 20

\begin{tabular}{|c|c|c|}
\hline Magnitude of flood & 25 percent & 10 percen \\
\hline 10 -year flood & 18 & 90 \\
\hline 25 -year flood & 31 & 105 \\
\hline 50 -year flood & 39 & 110 \\
\hline
\end{tabular}

Although the figures in the above table are based on hypothetical rather than on real flood events, they do give some indication of the considerable errors possible, from chance alone, in frequency graphs based on shortterm records. A comparison of the lengths of record indicated by this table and those available for Florida streams (table 1, p. 6) suggests that few records in Florida are long enough to provide reliable individual frequency graphs for floods of infrequent occurrence.

\section{Flood frequency by combining records}

As pointed out in the preceding section, the greatest obstacle in accurately defining the flood-frequency graph at a gaging station is shortness of record--a deficiency that can be corrected only by collecting additional records in future years. Thus, some other analytical method is necessary if more dependable information is to be extracted from the flood records available at the present time. One method commonly and profitably used in such circumstances is the combining of records, on the premise that the average answer obtained from all the records is more reliable than an answer from any single record. This section describes how flood records can be combined to serve two purposes: to improve the dependability of the frequency graph at the gaging stations, and to provide a method for determining flood frequencies at points away from gaging stations.

To accomplish these purposes the records are combined by two different methods. By the first method, the records are combined on the basis of a geometric similarity of the individual frequency graphs. Inspection of plotted frequency graphs for many gaging stations has shown that a close similarity in slope and relative shape of the graphs commonly exists over broad regions. If all gaged streams within a certain region show frequency graphs of the same general shape and slope, it may logically be concluded that in some respects that region is homogeneous as to flood-frequency characteristics, and that the average shape of the frequency graph as defined by gaged streams will also apply to ungaged streams in that region. Thus, by combining records on the basis of geometric similarity of the frequency graph, those regions for which the shape of the frequency graph is common to all streams, may be accurately determined.

The shape of the frequency graph, however, is a dimensionless property, and frequency graphs of which only the shape is known cannot be used to determine flood magnitudes in absolute terms at specific points on streams. The first method of combining records locates the frequency graph only with respect to the scale of recurrence intervals. To be of practical use, the graph must also be located with respect to the scale of magnitudes, that is, the dimensions of the graph must be restored.

This is accomplished by the second method of combining records, which relates groups of gaging stations displaying a similarity in their frequency graphs along the scale of magnitude. Experience has shown that in many parts of the United States, including the Southeastern States, frequency graphs for gaging stations can be used to outline areas in which streams draining basins of the same general physical characteristics, particularly of the same size, will produce essentially the same discharge for any given flood frequency. The second method of combining records serves to define these hydrologically similar areas by relating records on the basis of basin properties and the discharge, in absolute terms, for some arbitrarily selected recurrence interval. It further serves to define for any stream within such an area one point on the frequency graph with respect to both magnitude and frequency. If one point on the graph is thus defined, and the shape of the graph has been determined, the complete flood-frequency graph can readily be drawn.

It should be pointed out that the geographical boundaries of the areas defined by the two methods of combining records need not, and generally do not, coincide. To distinguish between the two classes of areas, those defined by the first method are referred to in this report as "flood regions" and those defined by the second method as "hydrologic areas".

\section{Combining records by geometric similarity of frequency graphs}

Before records for a number of gaging stations can be combined by this method, they must represent a homogeneous group both with respect to time and to geometric similarity.

8/ Gumbel, E. J., Floods Estimated by the Probability Method: Eng. News Rec., June 14, 1945. 
For time, the records to be combined are conveniently reduced to the same basis by using a common period for all records. Since length of record is of primary importance in a frequency graph, the base period selected should be as long as feasible. Records shorter than the base period are extended to that length by either of two methods. The first method is by estimating annual floods for years of no record from nearby stations having complete record. Order numbers are then assigned to all annual floods, observed or estimated, within the base period at each station. The estimated floods serve only to determine the proper order numbers for observed annual floods and otherwise do not enter into subsequent computations. The alternate method is to compute the mean annual flood for the actual period of the short-term station. Then it can be adjusted to the base period by using the ratio of the mean flood for the two periods at nearby long-term gaging stations.

For geometric similarity, the records to be combined may be regarded as a homogeneous group if the frequency graphs for the individual stations have approximately the same shape and slope. In comparing these graphs to ascertain whether this condition exists, it is convenient to compute the annual floods at each station as ratios to the station mean, thereby reducing all floods to dimensionless terms.

\section{Computation of mean annual floods}

The mean of the annual floods is commonly, though somewhat ambiguously, referred to as the "mean annual flood" which term, by virtue of convention, has been retained in this report. The mean annual flood is thus not to be confused with the flood having a recurrence interval of one year. According to the theory of extreme values as applied to floods by Gumbel, the mean of all the annual peak floods in a long-term record would have the magnitude of the flood of 2.33-year recurrence interval. The mean annual flood in this report was determined graphically from the individual station data as the intersection of the visually best-fitting frequency graph with the line corresponding to the 2.33-year recurrence interval. With occasional exceptions, experience has shown that the mean annual flood is indicative of the flood characteristics of a drainage basin and is therefore a good index on which to base comparisons.

\section{Test for geometric similarity}

Because flood records are random samples, a group of records can be tested for geometric similarity by determining whether the differences in slope of the individual frequency graphs for the lengths of record available are no greater than might result from chance alone. To test a group of records for this property the ratio of the 10 -year flood to the mean annual flood is first determined for each gaging station. These 10 -year flood ratios are then averaged to obtain a mean 10 -year flood ratio for the group. The mean annual flood at each gaging station is then multiplied by the mean ratio and the corresponding recurrence interval determined from the station frequency graph. These recurrence intervals are plotted against the number of years of record on a test graph, as shown in figure 5, based on the theory of extreme values. If the points for all the stations plot at random between the two curves, the group of records may be regarded as homogeneous and may be combined to define a flood region. Points plotting outside the envelope or grouped near the boundaries indicate where further subdivision is required.

\section{Regional frequency graph}

All station records satisfying the above test are used as a group to define an average or composite frequency graph for that region. From the flood-frequency graph for each gaging station, the flood discharge is determined for selected recurrence intervals $(1.1,1.5,2.33,5.0,10,25$, and higher if curve is defined). These floods for each gaging station are then reduced to dimensionless terms by expressing them as ratios to the mean annual flood (2.33-year recurrence interval). Using all the station records in a homogeneous region, the median ratio is determined for the flood of each selected recurrence interval. The median ratios plotted against the corresponding recurrence intervals define the regional composite frequency graph, which is based on all significant flood data available and may be considered to represent the most likely flood-frequency relation for all streams in the region. As a composite frequency graph expresses floods of other frequencies in terms of the mean annual flood, a complete frequency graph for any stream in the region can be drawn provided the mean annual flood is known.

\section{Combining records by similarity of mean annual floods}

As previously pointed out, the second method of combining records leads to the delineation of hydrologic areas within which a flood of some arbitrarily chosen magnitude and frequency can be related to the characteristics of drainage basins, thus fixing one point on the frequency graph. From the foregoing discussion it is now apparent that the most suitable flood for this purpose is the mean annual flood.

The selection of suitable basin characteristics for this basic relation is not so self-evident. Many physical characteristics of drainage basins are recognized as influencing the mean annual flood. Some exert a major influence, some a minor one. Some are susceptible of accurate definition and appraisal, some are not. For the sake of simplicity, the number of such factors considered should be as few as possible without detracting seriously from the accuracy of the final result. At the same time, their numerical values must be readily determinable from a vailable maps. 
- Factors influencing the mean annual flood

Among the many physical characteristics of drainage basins that affect runoff (and consequently the mean annual flood), those most applicable in Florida are size of drainage area of the basin; a mount of storage capacity in stream channels, swamps, and lakes; shape of basin; land and stream slopes; porosity of soil; type of vegetal cover; and land use, particularly as related to the existence of canal systems and pumping stations for drainage or irrigation.

Of these basin characteristics, the size of the drainage area is the dominant factor influencing the mean annual flood. Also of major importance on the Peninsula is the effect of storage in the numerous lakes in the drainage basin. This latter effect produces attenuated flood peaks. The attenuation, or reduction in flood peaks, is closely related to the percentage of lake area within the basin, a factor which can be readily determined from available maps.

\section{Hydrologic areas}

As a final step in combining records, the mean annual floods for all gaging stations are plotted against the corresponding drainage areas. For a drainage basin containing lake areas that are a significant percentage of the total drainage area, the mean annual flood has been adjusted for attenuation before correlating with the size of drainage area. Plotted data that indicate definite trend lines and also can be related geographically, are separated into groups. The geographic boundaries of the areas represented by these



Figure 5. --Homogeneity test graph. grouped data are based largely on an appraisal of the land factors influencing floods, as climatic factors will generally show little variation. Much of the scattering of the plotted data is due to the influence of factors other than the size of drainage area and storage in lakes. However, in lieu of attempting to evaluate these factors, the State was subdivided into several additional hydrologic areas and fairly satisfactory correlations with drainage area have been obtained. For each of these hydrologic areas a curve relating mean annual flood and drainage area is prepared. An auxiliary curve is also prepared for defining the attenuation of flood peaks in applicable areas. Because these curves average the data for gaged points on streams in the area, they may be regarded as the most likely relations for ungaged points. Thus the only factors required to determine the mean annual flood at a desired point on a stream are the drainage area and the area of contributing lakes upstream.

\section{FLOOD FREQUENCY IN FLORIDA}

On the Peninsula of Florida, north of Lake Okeechobee and excluding the St. Marys and Suwannee River basins, practically no streamflow records were collected prior to 1930 . However, several gaging stations were established in this region during 1930, generally on the larger streams, and there is available a fairly adequate network of stations on which to base frequency studies for the 24-year period, 1930-53. In the region including the St. Marys and Suwannee River basins and extending westward for the remainder of the State, records from a few gaging stations extend back to 1927 and the base period used for this region is the 27-year period, $1927-53$.

\section{Flood regions of Florida}

On the basis of the test for geometric similarity, two regions in Florida were indicated for which the characteristics of annual flood peaks were more or less homogeneous. These homogeneous flood regions are designated as regions $A$ and $B$ in figure 6 , and on plate 1 . For the extreme southern part of the Florida peninsula, South of Lake Okeechobee, the available data are inadequate for flood-frequency studies. This region will be discussed in more detail in a following paragraph.

Records for several gaging stations in regions $A$ and $B$ have not been used to define the flood-frequency relationships because of regulation and diversions, interconnection of the drainage basins, proximity to other stations, or short periods of record. 


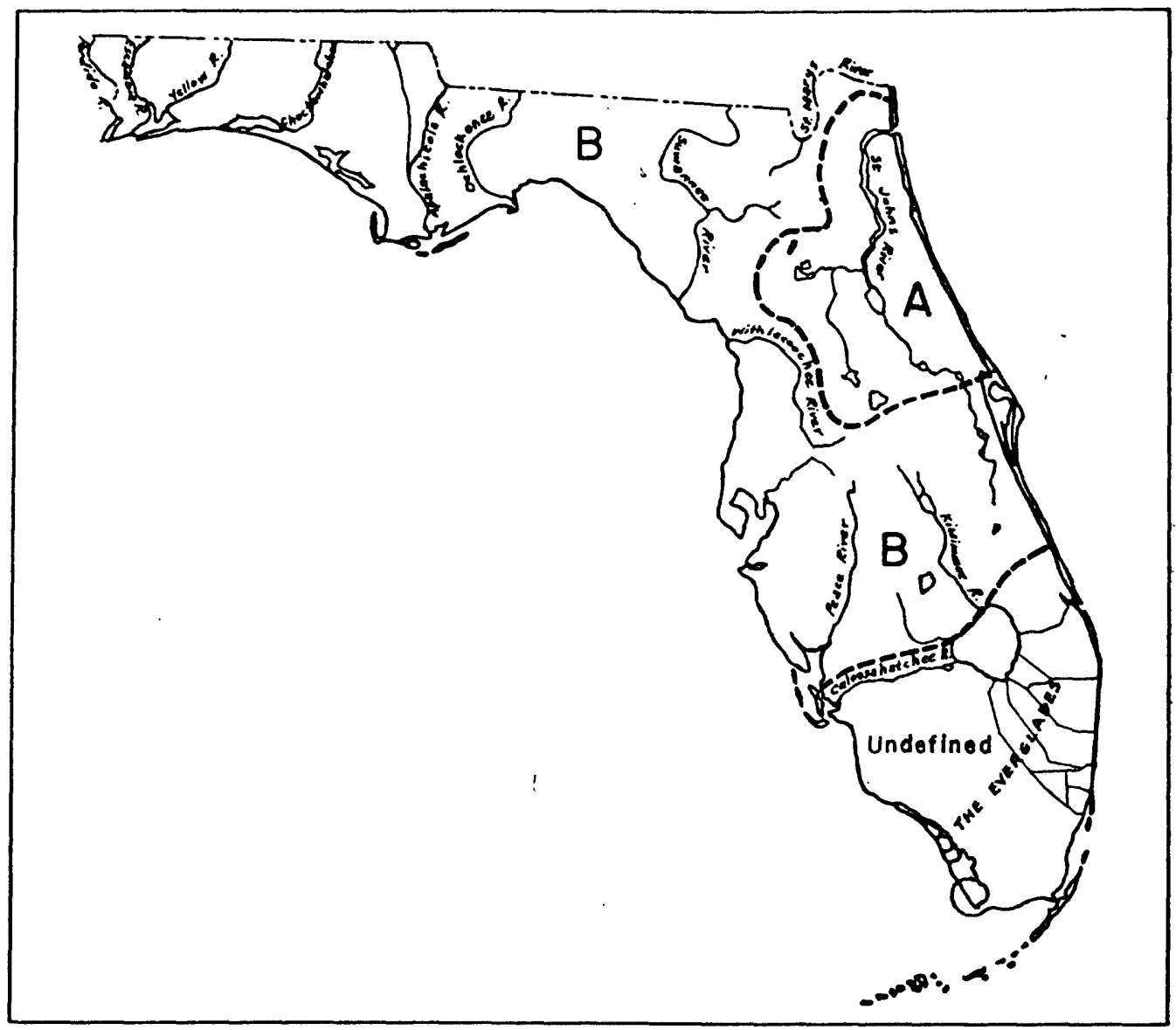

Figure 6. --Homogeneous flood regions of Florida.

Delineation of flood regions

Region A. -- This region includes the lower part of the St: Johns River basin and the adjacent small coastal streams draining into the Atlantic Ocean. There are 11 gaging stations in this region that have sufficient length of discharge records on which the composite flood-frequency was based. Of these stations, only one is complete for the 24-year base period, 1930-53. Three other stations cover a term of 20 years or longer. By correlation, however, the period of annual flood peaks for each of the 11 stations was extended to the same 24 -year base. The composite flood-frequency graph for region $A$ is shown as figure 7.

Region B. - - This region covers practically the entire State except region $A$ and the area south of Lake Okeechobee. In central Florida practically no discharge records were collected before 1930 and little information on historical floods could be obtained before the beginning of record. However, in the northern part of the peninsula and in West Florida a few discharge records extend back as far as 1927. Flood data for outstanding historical floods at several stations are available for a number of years antedating the period of record.

In this region there are 70 gaging stations with sufficiently long discharge records on which to base the composite flood-frequency relationship. Of these 70 gaging stations, records for only 4 are complete for a $27-y e a r$ period, 1927-53, and 7 are complete for a 24-year period, 1930-53. However, by correlation by either of the two methods previously described and by use of historical data for several stations a composite flood-frequency graph based on the 70 gaging station records in region $B$ has been defined. This composite flood-frequency graph is shown in figure 8 and has been extended to the 50-year recurrence interval with reasonable assurance.

Records for a number of stations in southern Georgia and southern Alabama were included for additional definition of the composite flood-frequency relationship for this region. The relationship for region $B$ in $F$ lorida differs slightly from that applicable for region $D$ in Georgia, as shown in Circular 100, and region $B$ in $A$ labama as shown in Circular 342, but is approximately the same as the average of the two. The relationship as shown in figure 8 is the best fit of the data for Florida gaging stations and is therefore considered applicable for conditions in Florida.

Records of annual flood peaks for the Apalachicola River at Chattahoochee have been available since 1919. The station-frequency curve for the 35-year period, 1919-53, has been included as figure 9 in this report. 


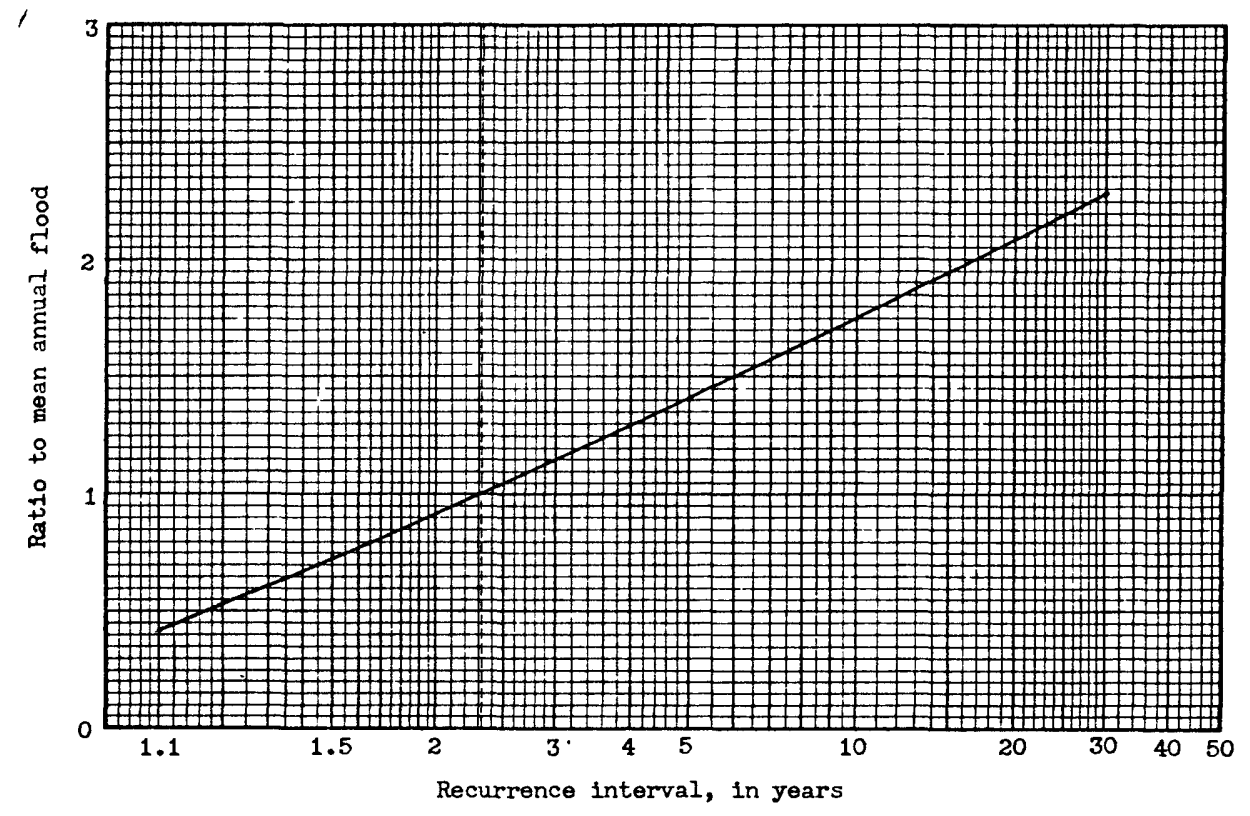

Figure 7. --Composite frequency curve for floods in region A.

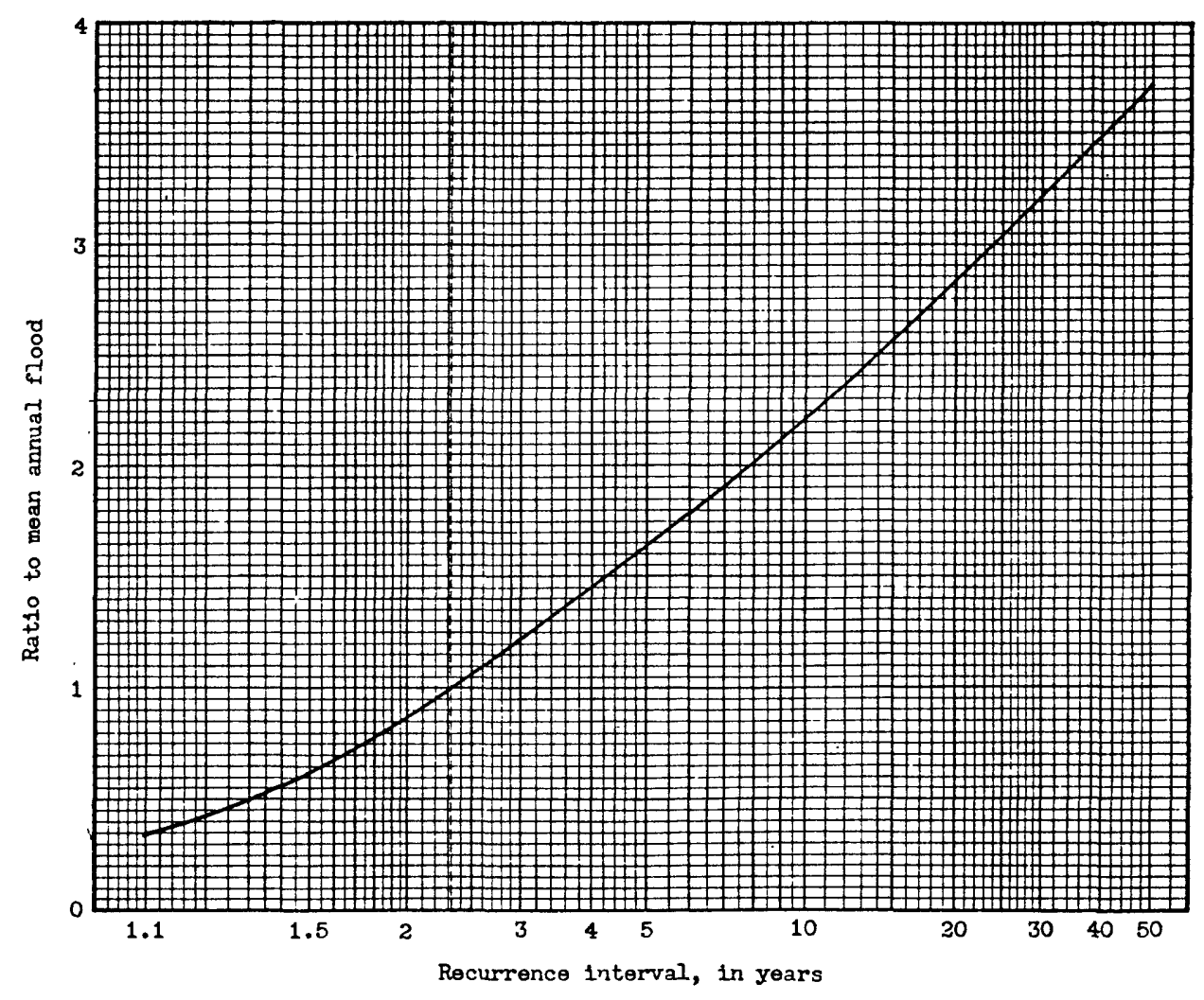

Figure 8. --Composite frequency curve for floods in region $B$. 


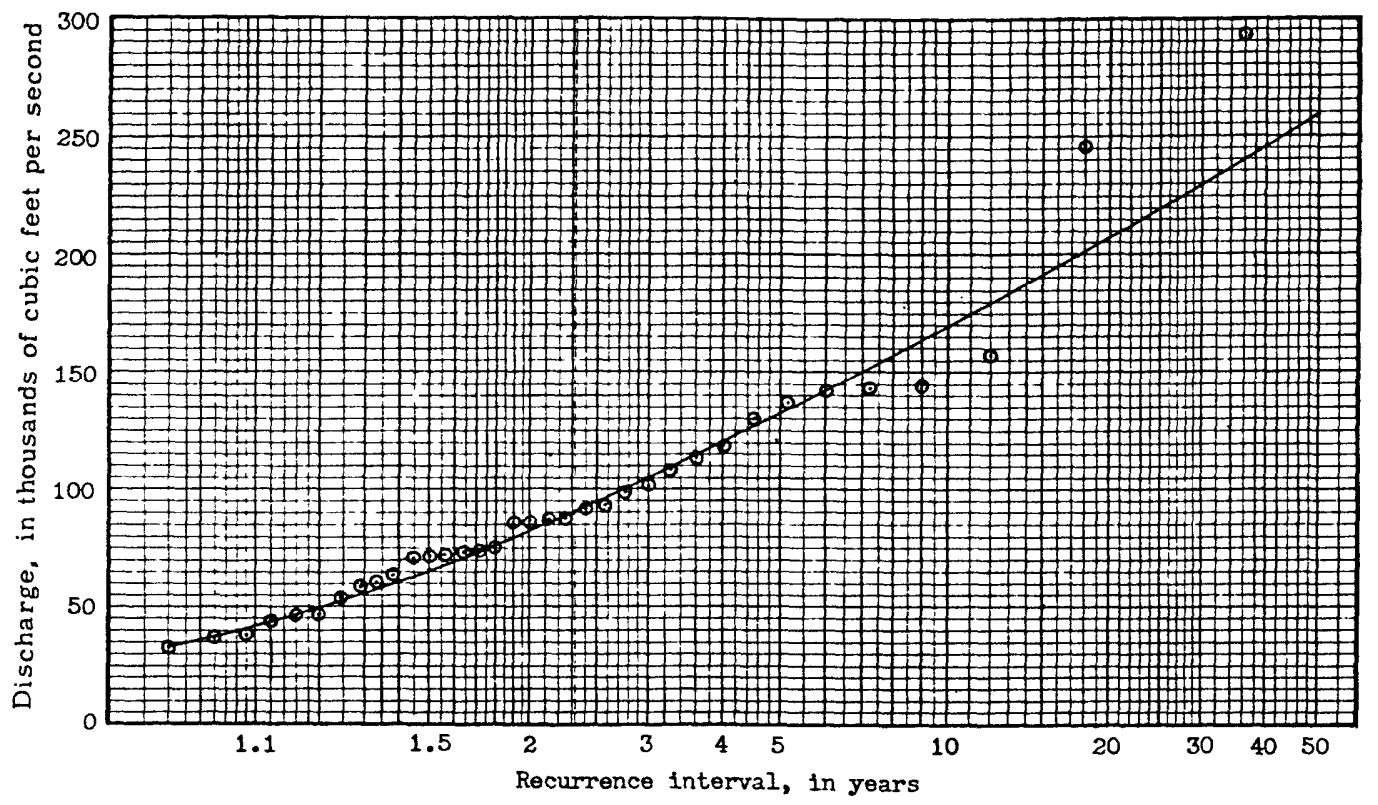

Figure 9. --Frequency of annual floods, Apalachicola River at Chattahoochee, Fla.

Southern Florida. --Drainage in the area south of Lake Okeechobee is mainly by means of interconnected canal systems which are progressively being improved and developed. The regimen of flow in these canals is thus being continually altered. Practically no streamflow data had been collected in this area before 1940. Because of the short period of record, the progressive changes in the canal systems, regulation, and diversions, the flood frequency of this region is indeterminate. The annual flood peaks for several main channels have been included in this report in order to show the magnitude and variation of floods at selected sites.

The composite flood-frequency graphs of figures 7 and 8 are basic to the remainder of the report in that they are derived from all significant flood data and are presumed to represent the most probable relation between flood magnitude and frequency for any stream in the regions to which they apply. The graphs are of general applicability because they express flood discharges as ratios to the mean annual flood. Thus, to derive a frequency graph for any stream, expressing flood discharges in cubic feet per second, it is only necessary to multiply the mean annual flood, in cubic feet per second, for that stream by the ratios indicated by the appropriate composite graph. The remainder of the analytical portion of this report is therefore largely devoted to methods of determining the mean annual flood for streams in Florida.

\section{Hydrologic areas of Florida}

The base data used for determining the hydrologic areas in Florida are listed in table 1, which shows the drainage area and mean annual flood for applicable base periods for gaging stations on streams in flood regions $A$ and $B$. As previously discussed in this report, flood frequencies and mean annual floods on streams and canals in southern Florida cannot be evaluated on the basis of available data. Excluding southern Florida, the remainder of the State has been divided into seven areas in each of which the base data indicate a similarity in hydrologic conditions relating to floods. For these hydrologic areas, the relationship between mean annual flood and drainage area has been defined. Many characteristics, other than size of the drainage area, influence the mean annualflood. The dominant secondary influence in Florida is the amount of storage capacity in lakes above a gaging station. In order to eliminate the effect of storage in lakes, an attenuation factor has been computed for adjusting the mean annual flood for a drainage basin containing a significant percentage of lake area. The method of adjustment of mean annual floods for several gaging stations with attenuated flood peaks is shown in table 2 . The attenuation factor is shown in figure 10. In defining the relation of mean annual flood to drainage area for each hydrologic area the adjusted mean annual floods as shown in column 6 , table 2 , has been used in conjunction with mean annual floods for drainage areas devoid of lakes. To compensate for the less tangible factors that influence the mean annual flood, the State was subdivided into several hydrologic areas.

The 7 hydrologic areas of Florida are shown on plate 1. Because of inadequate data defining the areal boundaries, it was necessary to select these boundaries somewhat arbitrarily. In general, the boundaries follow drainage divides. In some areas, however, the boundaries cross stream lines. The crossings were placed at the junctions with large tributaries or lakes, where changes in the relation most logically occur. 
FLOOD FREQUENCY IN FLORIDA

Table 2. --Adjustment of mean annual flood for attenuation by storage in lakes

\begin{tabular}{|c|c|c|c|c|c|c|}
\hline $\begin{array}{l}\prime \\
\text { Sta. } \\
\text { No. }\end{array}$ & $\begin{array}{l}\text { Total drain- } \\
\text { age area } \\
\text { (sq mi) } \\
\text { (1) }\end{array}$ & $\begin{array}{l}\text { Surface area } \\
\text { of contri- } \\
\text { buting lakes } \\
\text { ( } \mathrm{sq} \mathrm{mi)} \\
\text { (2) }\end{array}$ & $\begin{array}{l}\text { Ratio of lake } \\
\text { area to total } \\
\text { drainage area } \\
\text { (Col. } 2 \div \text { Col. 1) } \\
\text { (3) }\end{array}$ & $\begin{array}{l}\text { Mean annual } \\
\text { flood } \\
\text { (cfs) } \\
\text { (4) }\end{array}$ & $\begin{array}{c}\text { Discharge } \\
\text { ratio from } \\
\text { Fig. } 10 \\
\text { (5) }\end{array}$ & $\begin{array}{c}\text { Adjusted mean } \\
\text { annual dis- } \\
\text { charge (cfs) } \\
\text { (Col. } 4 \div \text { Col. } 5 \text { ) } \\
\text { (6) }\end{array}$ \\
\hline 24 & 160 & 22.1 & 0.138 & 285 & 0.18 & 1,580 \\
\hline 25 & 640 & 128 & .200 & 420 & .091 & 4,620 \\
\hline 28 & 1,100 & 161 & .146 & 1,080 & .16 & 6,750 \\
\hline 37 & 431 & 55.4 & .129 & 690 & .21 & 3,280 \\
\hline 52 & 31.0 & 8.3 & .268 & 66 & .068 & 970 \\
\hline 55 & 58.9 & 7.3 & .124 & 131 & .23 & 570 \\
\hline 60 & 62.2 & 16.0 & 257 & 86 & .071 & 1,210 \\
\hline 62 & 385 & 29.6 & .077 & 2,900 & .60 & 4,830 \\
\hline 63 & 109 & 20.4 & .187 & 630 & .100 & 6,300 \\
\hline 65 & 624 & 93.1 & .149 & 1,030 & .15 & 6,870 \\
\hline 84 & 50.0 & 10.5 & .210 & 123 & .088 & 1,400 \\
\hline 85 & 150 & 15.8 & .105 & 877 & .35 & 2,500 \\
\hline 87 & 26.0 & 7. 3 & .280 & 43 & .065 & 660 \\
\hline 88 & 390 & 42.0 & .108 & 1,700 & .33 & 5,150 \\
\hline 89 & 840 & 45. 7 & .054 & 7,500 & .82 & 9,150 \\
\hline 90 & 1,370 & 45.7 & .033 & 10,000 & .93 & 10,800 \\
\hline 91 & 235 & 8.5 & .036 & 3,200 & .92 & 3,480 \\
\hline 97 & 10.0 & 1.3 & .130 & 57 & .20 & 285 \\
\hline 114 & 630 & 15.6 & .025 & 7,000 & .96 & 7,300 \\
\hline
\end{tabular}

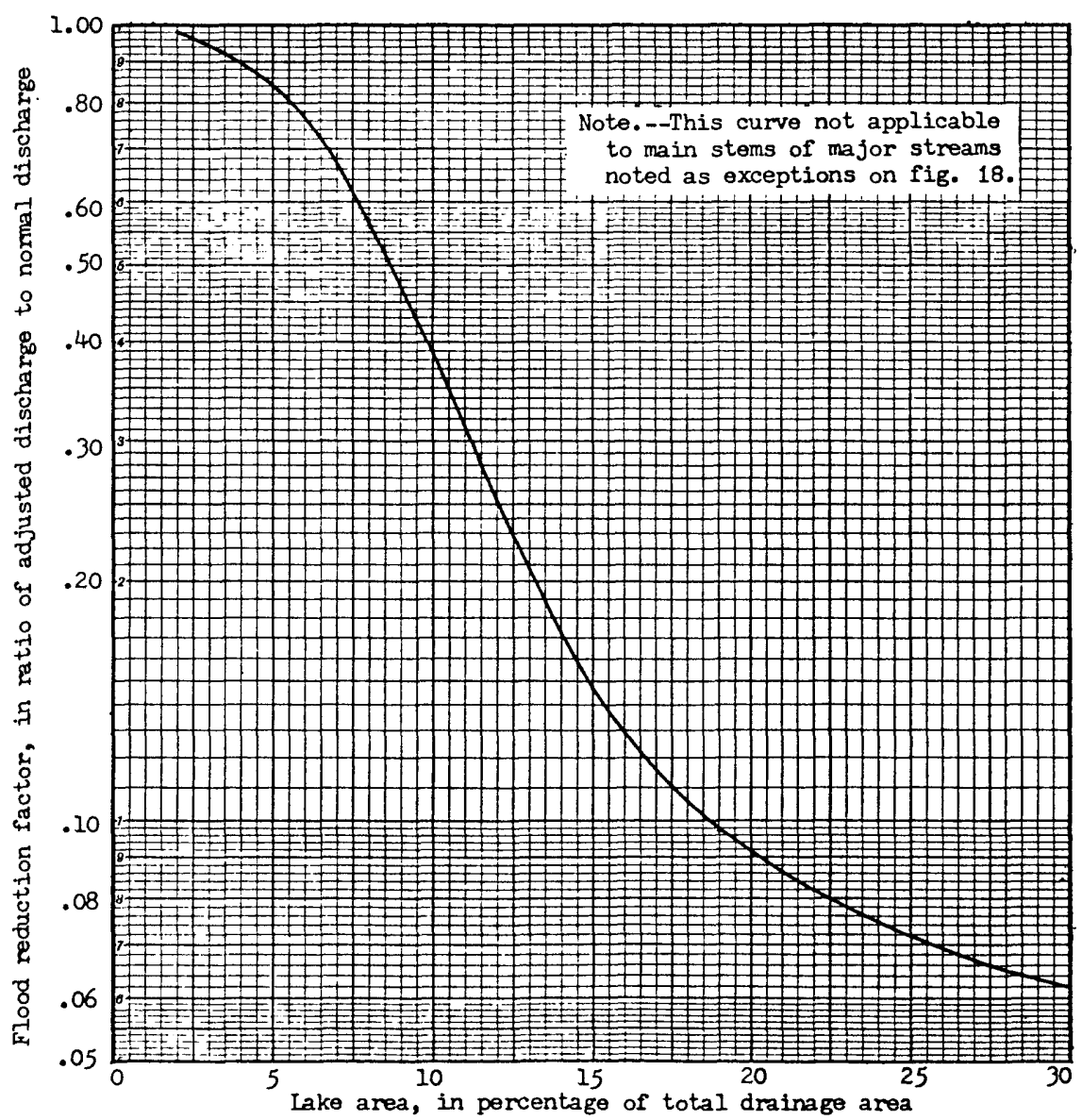

Figure 10. --Relation of lake area above gaging station to reduction of mean annual flood. 
Thes hydrologic areas are designated in numerical order, beginning in the northeast corner of : 2 e State.

Delineation of hydrologic areas

Area 1. - Except for small enclosed areas 2A, 2B, and 3, the entire central peninsula, east of the Suwannee Hiver and north of Lake Okeechobee, is included in area 1. Records of mean annual floods for 23 gaging stations on tributary streams were available for defining the relationship with drainage area. Flood peaks for several of these stations are attenuated by storage in lakes within the basin. Mean annual floods for these stations have been adjusted for a floods for stations not affected by storage in lakes to define the relation of mean annual flood to drainage area. This relation is shown as figure 11 and varies as the 0.70 power of the drainage area. The adjusted results of the

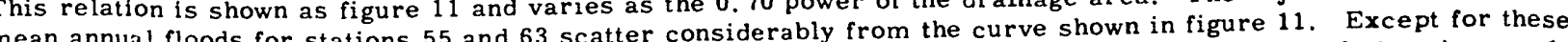
mean annual floods for stations 55 and 63 scatter considerably from the curve shown in figure 11 . Ex

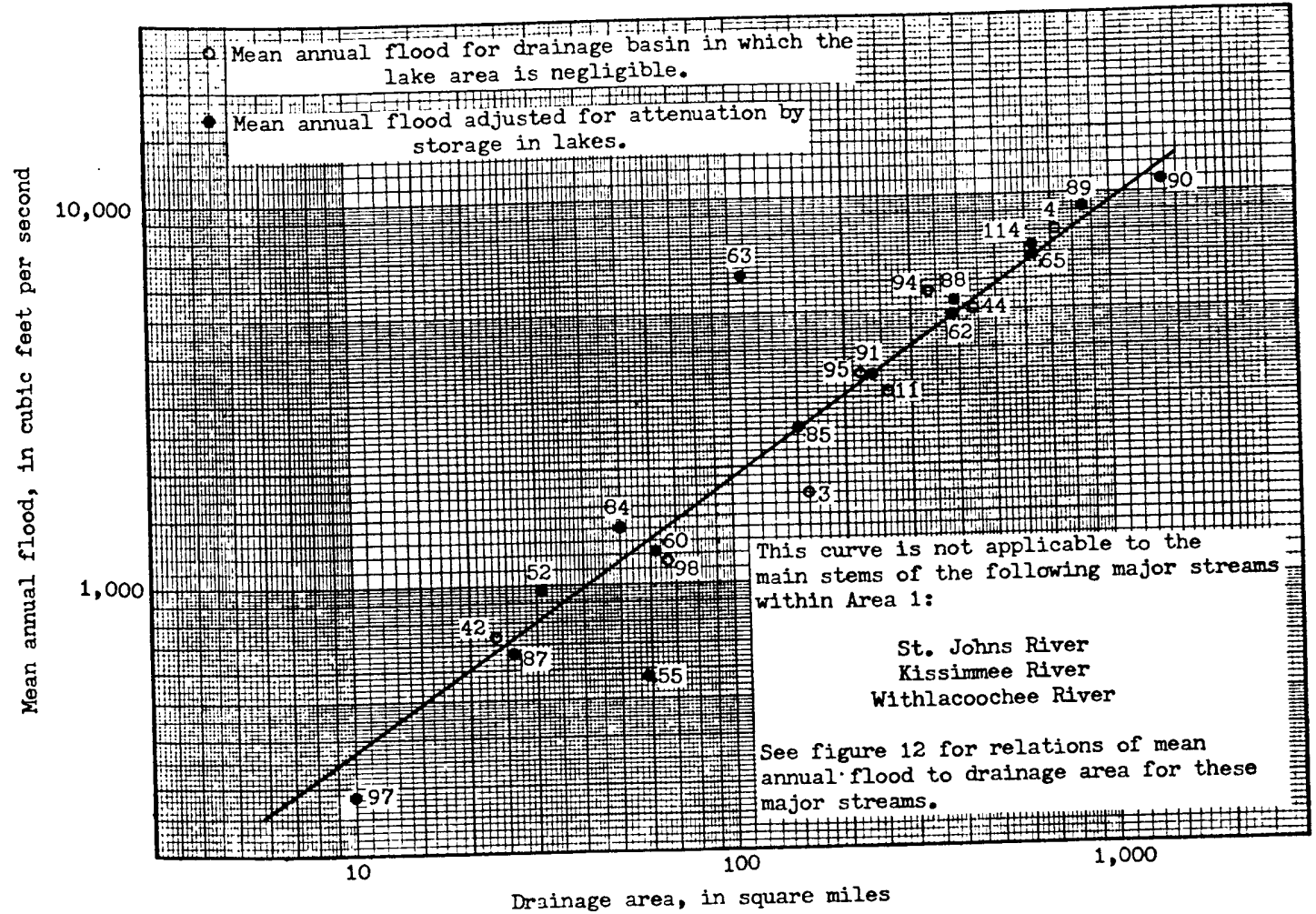

Figure 11. --Relation of mean annual flood to drainage area for area 1.

In computing the actual mean annual flood for an ungaged drainage area in area 1 , the value as obtained from figure 11 must be reduced for attenuation by storage in lakes if the lake area is a significant percentage of the total drainage area. The attenuation factor may be obtained from the relation shown in figure 10.

The relation shown in figure 11 is not applicable for the main stems of the major rivers in area 1. Refer to figure 12 for the relations of mean annual flood to drainage area for the St. Johns, lower Oklawaha, Kissimmee, and Withlacoochee Rivers.

Areas $2 \mathrm{~A}$ and $2 \mathrm{~B}$. - - A rea $2 \mathrm{~A}$ is the Black Creek basin and area $2 \mathrm{~B}$ includes the Manatee and Little Manatee River basins. The relation of mean annual flood to drainage area for these two areas is based on data for gaging stations $40,41,92$, and $93--$ two stations in each area. The two areas a re a considerable distance apart, but the same relation, as shown in figure 13 is applicable within the limits defined for both areas. The curve defining the relation for areas $2 \mathrm{~A}$ and $2 \mathrm{~B}$ varies as the 0.70 power of the drainage area.

A rea 3. - - This area includes the Oklawaha River basin in central Florida. The mean annual floods adjusted for attenuation by storage in lakes for stations $24,25,28$, and 37 define the relation with drainage, area for he Oklawah basin as hown in figure 13. This relationship, which varies as the 0.70 power of the drainage area, the Oksed entirely as shown in figure 13. This relationship, which a value of discharge, estimates of mean annual floods for an ungaged drainage area must be reduced by the attenuation factor. 


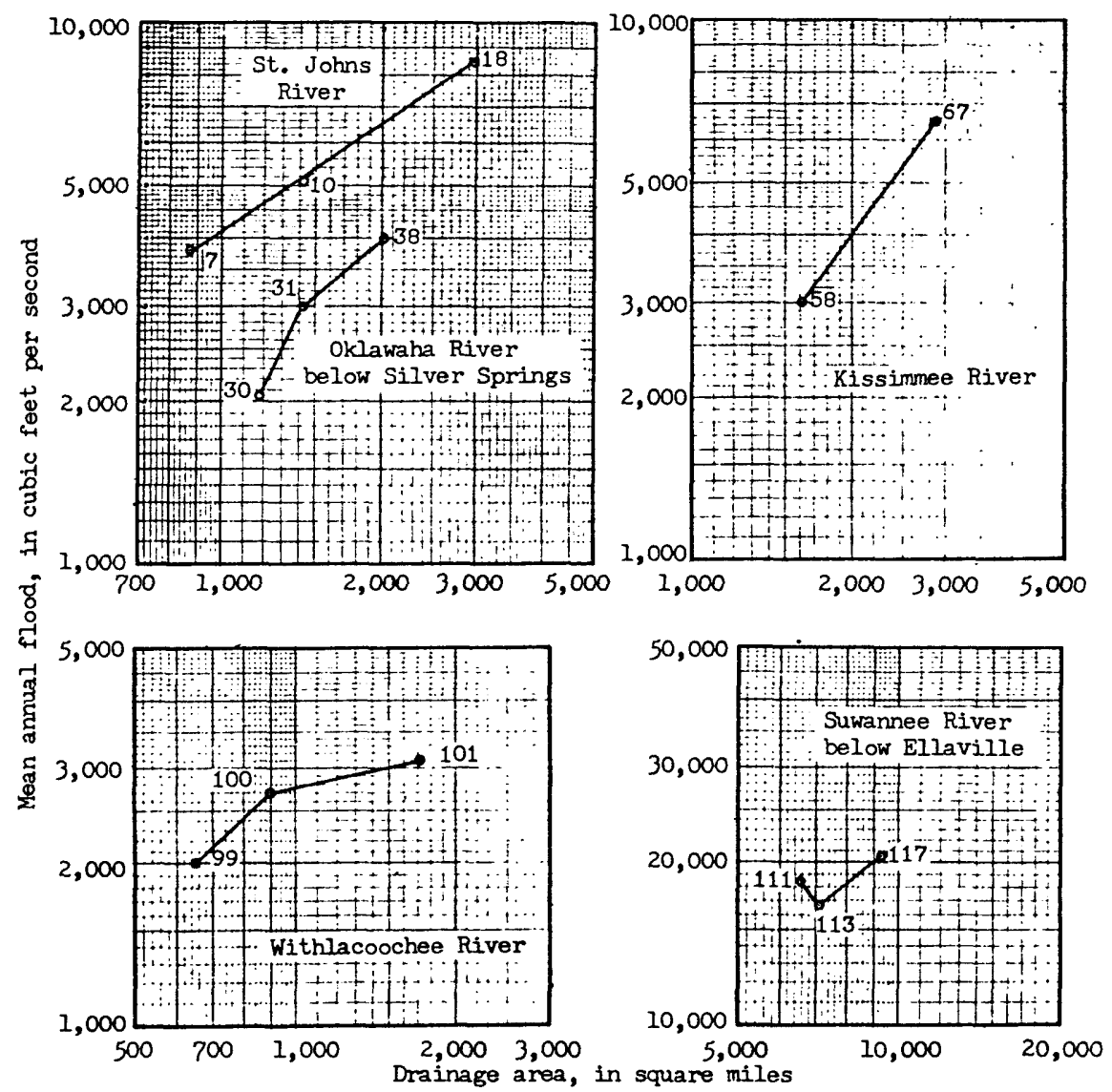

Note: Data unavailable for defining mean annual flood along Apalachicola River. Mean annual flood at Chattahoochee (drainage area, 17,100 sq mi) is 90,000 cubic feet per second (see fig. 9 ).

Figure 12. --Relations of mean annual flood to drainage area along main stems of large rivers in Florida.

The relation for area 3 as shown in figure 13 is not applicable to the main stem of the Oklawaha River below Silver Springs. Refer to figure 12 for the relation applicable to this reach of the river.

Area 4. - - This area includes the Suwannee River basin, except for station 114 in area 1 , and stations 108 and 109 in area 5 . The western boundary of the area is indefinite, owing to an inadequate network of gaging stations on the small coastal streams between the Suwannee and Ochlockonee Rivers. Because of a lack of any conclusive evidence, the western boundary of area 4 has been arbitrarily drawn along the eastern watershed boundary of the Aucilla River.

The relation of mean annual flood to drainage area for the upper Suwannee River and tributaries are based on data for stations 104,105, 106,107, 110,115,116, and 119, some of which are in Georgia. This relation is shown in figure 14. The curve has a slope of 0.70 . The mean annual flood for Fenholloway River at Foley, station 119 , is somewhat lower than that obtained from the relation curve. However, an extension of the lower end of the curve as a straight line with a slope of 0.70 is considered to be more reliable than that indicated by the single gaging station with a short-term record. The relation as shown in figure 14 is the best estimate of mean annual floods of ungaged streams in area 4 in Florida. In Geological Survey Circular 100, "Floods in Georgia, Frequency and Magnitude," the hydrologic areas were delineated somewhat differently and the relation curves were drawn to give the best results for Georgia, based on gaging stations in Georgia. Even though some of the Georgia gaging station data were used in this report and area 4 is adjacent to Georgia, the relation curve as defined in this report is the best fit of the data for Florida gaging stations and no adjustments were considered in order to obtain better conformity. 


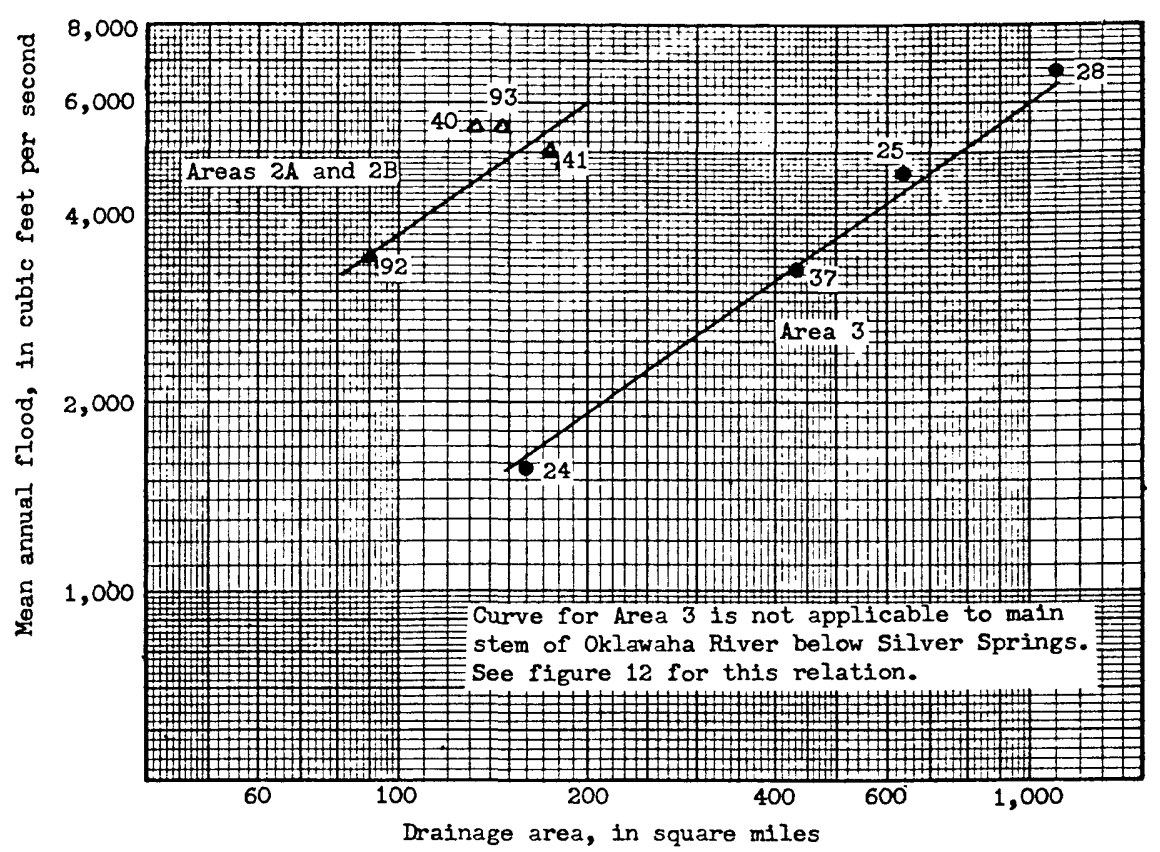

Figure 13, --Relation of mean annual $f$ lood to drainage area for areas $2 \mathrm{~A}, 2 \mathrm{~B}$, and 3 .

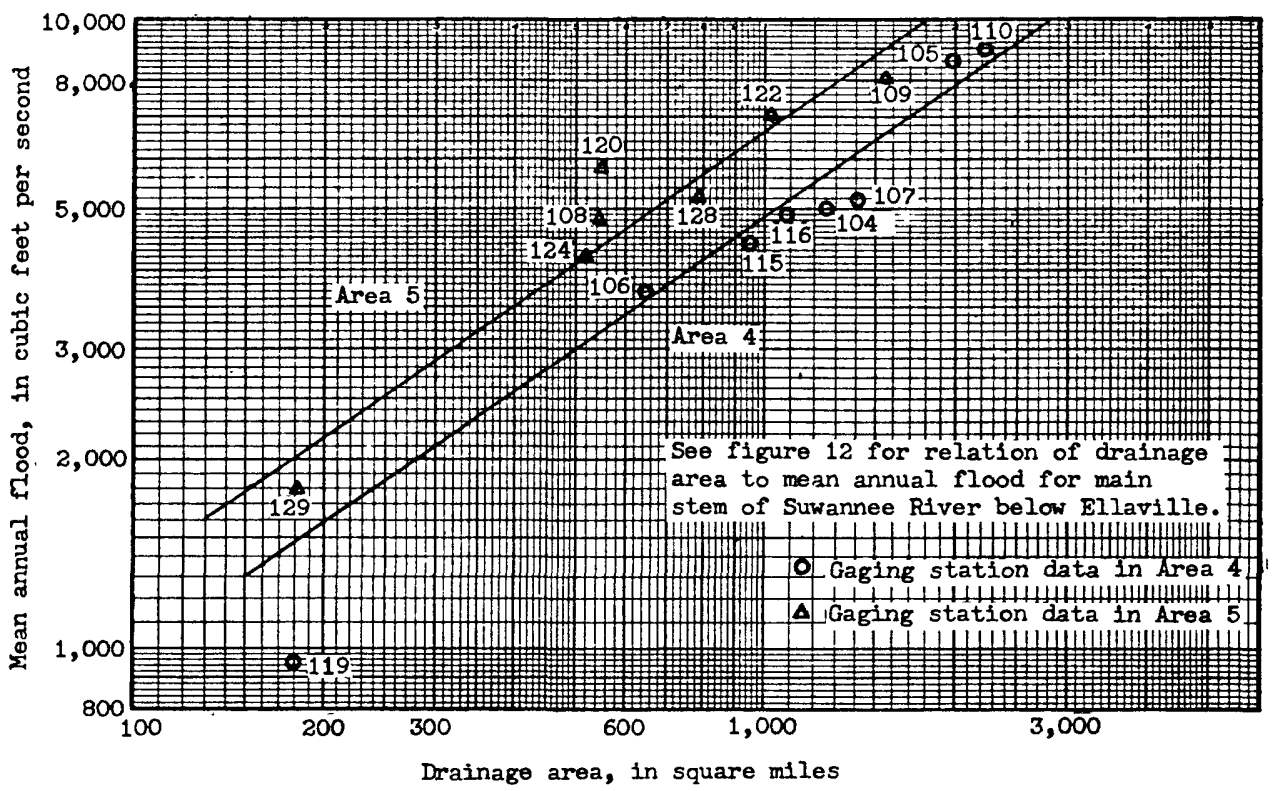

Figure 14. --Relation of mean annual flood to drainage area for areas 4 and 5.

Gaging stations 111,113 , and 117 on the main stem of Suwannee River below the confluence of the Withlacoochee River at Ellaville define flood characteristics slightly different from those of the smaller tributaries. A plot of the mean annual flood versus drainage area for these stations is shown in figure 12 and provides the best basis for determining the mean annual floods at ungaged points along the main stem.

Area 5. - - That part of northern Florida extending from the Aucilla River basin to the eastern watershed boundary of the Choctawhatchee River is designated as area 5. The relation of mean annual flood to drainage area is based on stations 108,109,120,122,124,128, and 129, and is shown in figure 14. This curve has a slope of 0.70. 
Station 120 is in the upper Ochlockonee River basin in Georgia and the plotted data for this station show considerable departure from the relation curve. As indicated in the discussion above for area 4 , the relation curve is more applicable to conditions in Florida and therefore its location is more influenced by data from Florida gaging stations.

Records of discharge have been collected at only one station on the Apalachicola River below the confluence of the Flint and Chattahoochee Rivers. The individual flood-frequency curve for this station is shown in figure 9. Mean annual flood for this station may be obtained from the frequency curve. Except for a long-term stage record at station 127, near Blountstown, there is no record on which to base any regional or composite relationships for the main stem of this river.

Area 6. --This area is drained by the Choctawhatchee River which flows in a southward direction from A labama through Florida. Data for two stations, 132 and 133, in Florida and for several upstream stations in Alabama are available for defining the relationship between mean annual flood and drainage area. This relation is shown by the curve in figure 15. The slope of the curve is 0.70 . Data for only two of the stations in Alabama, stations 130 and 131, are repeated in this report. Also from Geological Survey Circular 342, "Floods in Alabama, Magnitude and Frequency," data for two additional upstream stations were obtained for defining the lower limits of the curve. No flood data are available from small drainage areas in this section of Florida.

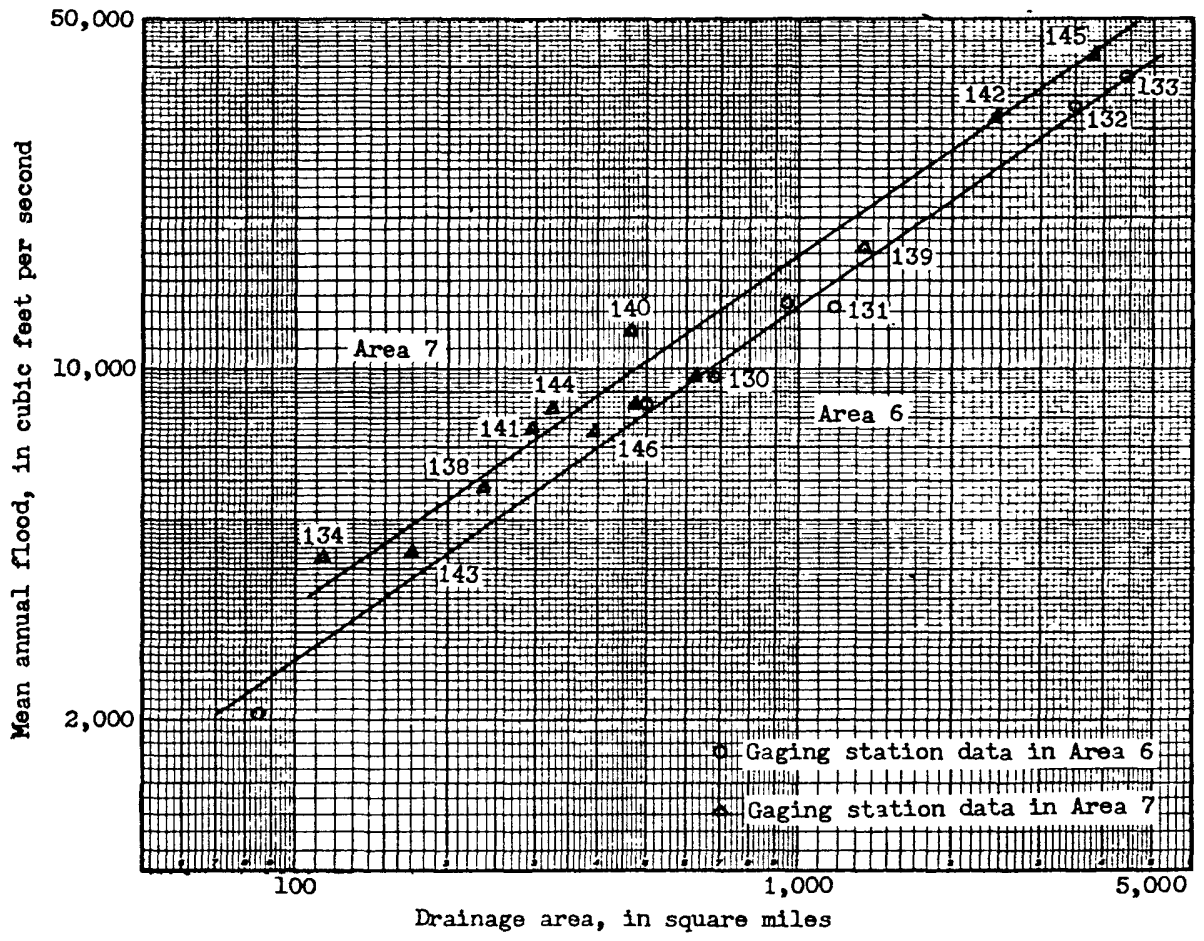

Figure 15. --Relation of mean annual $f$ lood to drainage area for areas 6 and 7.

Area 7. --This area includes the extreme northwestern part of Florida. The principal streams draining the area are the Yellow River, Blackwater River, Escambia River, and Perdido River. The relation between mean annual flood and drainage area is based on data from stations 135, 136, 138, 145, and 146 in Florida and several additional headwater stations in Alabama. The relation curve for this area is shown in figure 15 . The slope of the curve is $0.70--$ the same as defined for each area in the State.

\section{Frequency of annual flood stages}

The foregoing analyses and discussion of flood frequencies pertain primarily to discharge data. In the coastal lowlands and particularly in Southern Florida where the land surface is low and flat and has little slope, stage is an important index of flood conditions.

In addition to those stations at which both stage and discharge records have been collected, several stations on the St. Johns and Kissimmee Rivers, have been operated for a number of years for the collection of records of stage only. Because of the importance of stage data on the main stem of these two rivers and the meager coverage by discharge stations, flood-frequency plots of annual peak stages have been included in this report. 
The base period for these stage-frequency analyses has been selected as the same 24-year period, 1930-53, as was used for the discharge-frequency studies. The methods of analysis and extension of all station records to the same base period are also the same.

\section{St. Johns River}

Annual flood stages from stations $5,6,7,8,9,10,12,16$, and 18 on the main stem of the St. Johns River were available for defining the frequency-of-stage plots. Records of highwater elevations of outstanding floods at station 16 extend back to 1871 but there is some doubt as to the reliability of these historical data andalso of the gage datum. Correlation with other stations is too unreliable to extend the recurrence interval beyond the 24-year base period, 1930-53. Mean sea level stage curves for floods of 2.33-, 5-, 10-, and 30-year recurrence intervals are plotted against miles above the mouth of the river and are shown in figure 16 . The curves in figure 16 have been drawn as straight lines connecting the plotted data, although the actual flood profile undoubtedly does not vary uniformly between stations. However, the approximate stages for various flood frequencies at ungaged sites along the main stem may be estimated from the curves shown in figure 16.

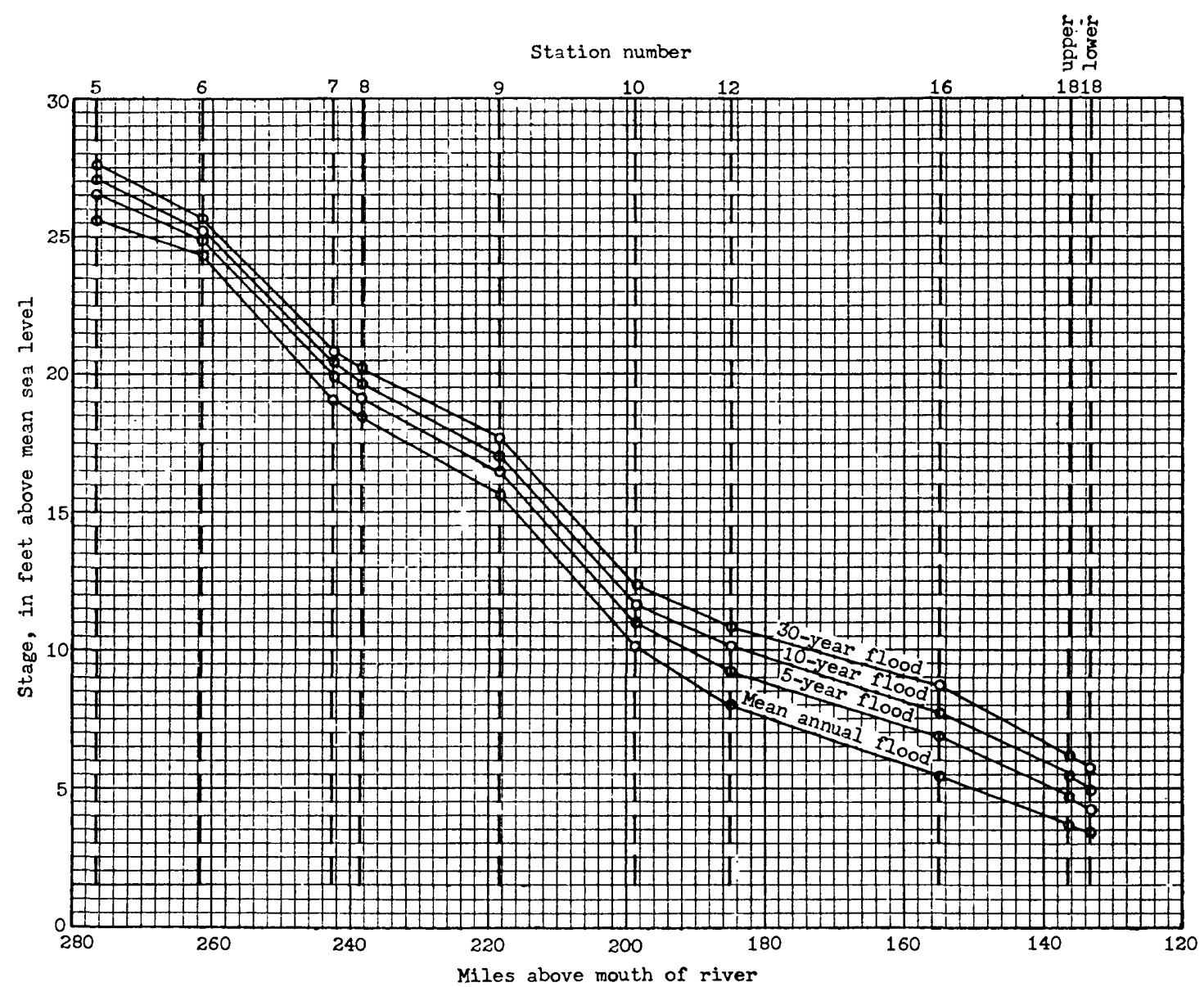

Figure 16. --Variation of mean annual, 5-, 10-, and 30-year flood stages with channel distance for main stem of St. Johns River, Florida.

Kissimmee River

Annual flood-stage records were available from stations $58,59,66$, and 67 on the main stem of the Kissimmee River; the stage-frequency relations were based on these records. Mean sea level stage curves for floods of $2.33-, 5-, 10-$, and 30 -year recurrence intervals are plotted against miles above the mouth of the river and are shown in figure 17 . This relation provides the best basis for estimates of stage for floods of various frequencies at ungaged sites along the main stem of the Kissimmee River. 


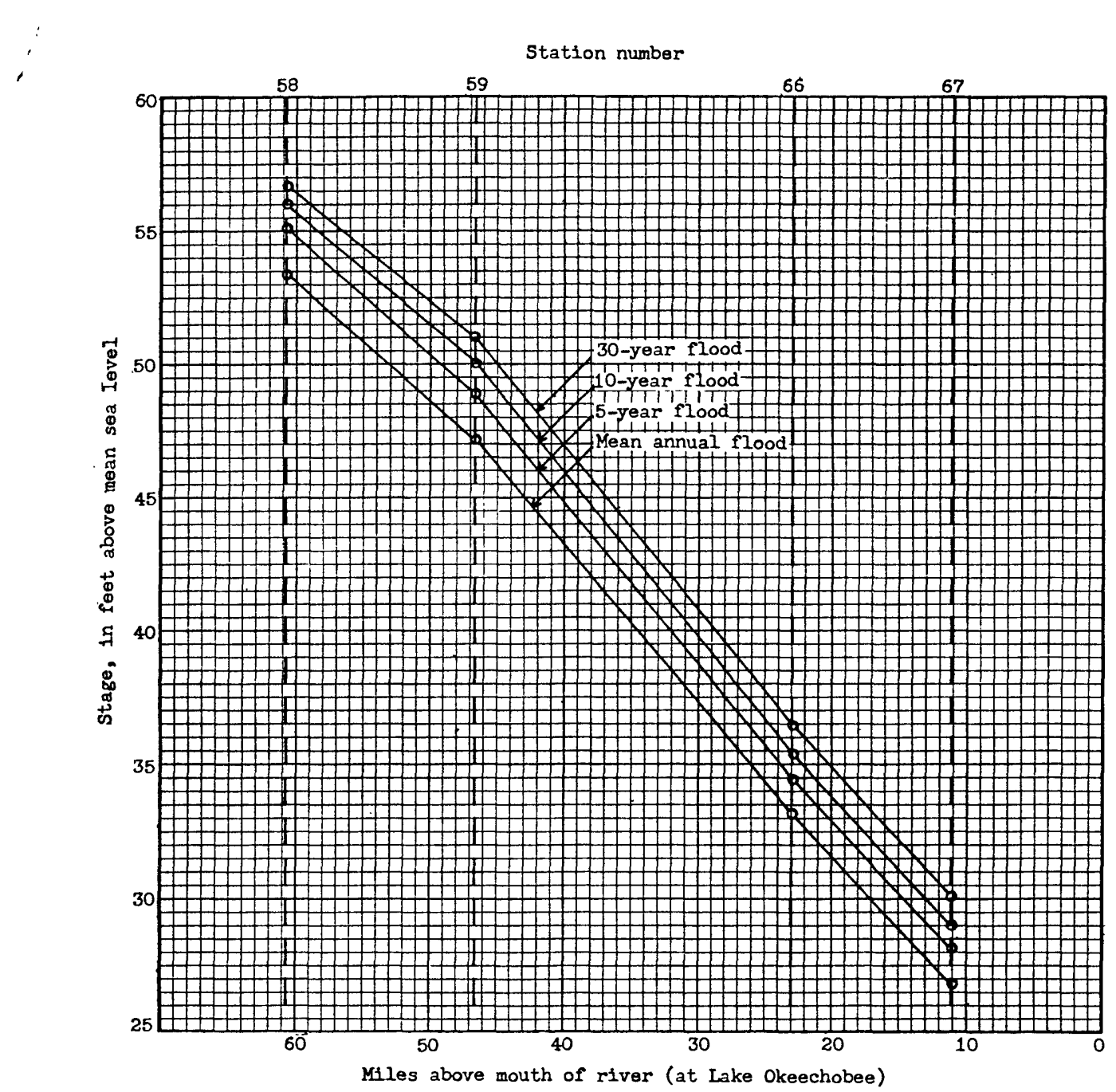

Figure 17. --Variation of mean annual, 5-, 10-, and 30-year flood stages with channel distance for main stem of Kissimmee River, Florida.

\section{Summary of flood-frequency relationships}

Composite frequency curves for floods with recurrence interval of 50 years or less have been developed for two flood regions in Florida. These flood regions, designated A and B, are shown in figure 6 and on plate 1 . The flood frequency curves have been developed by expressing flood magnitudes as ratios to the mean annual flood. Curves applicable to regions $\mathrm{A}$ and $\mathrm{B}$ are shown in figures 7 and 8 respectively. Flood data are inadequate for defining the flood-frequency relation applicable to southern Florida.

To estimate the mean annual flood in regions $A$ and $B, 7$ hydrologic areas have been delineated as shown in plate 1 , and in each area the mean annual flood has been expressed as a function of the drainage area. The curves of relation between mean annual flood and drainage area in these areas are shown in figures 11, 13-15, and are combined in figure 18. As shown by relations in figure 12 , the main stems of the major rivers do not have the same characteristics as the tributary streams. The relation of the mean annual flood along the main stem of these rivers has been shown as a plot of the mean annual flood against the drainage area. Such relations are required for the St. Johns River main stem, Oklawaha River main stem below Silver Springs, Kissimmee River main stem, the Withlacoochee River main stem, and the lower Suwannee River main stem. 


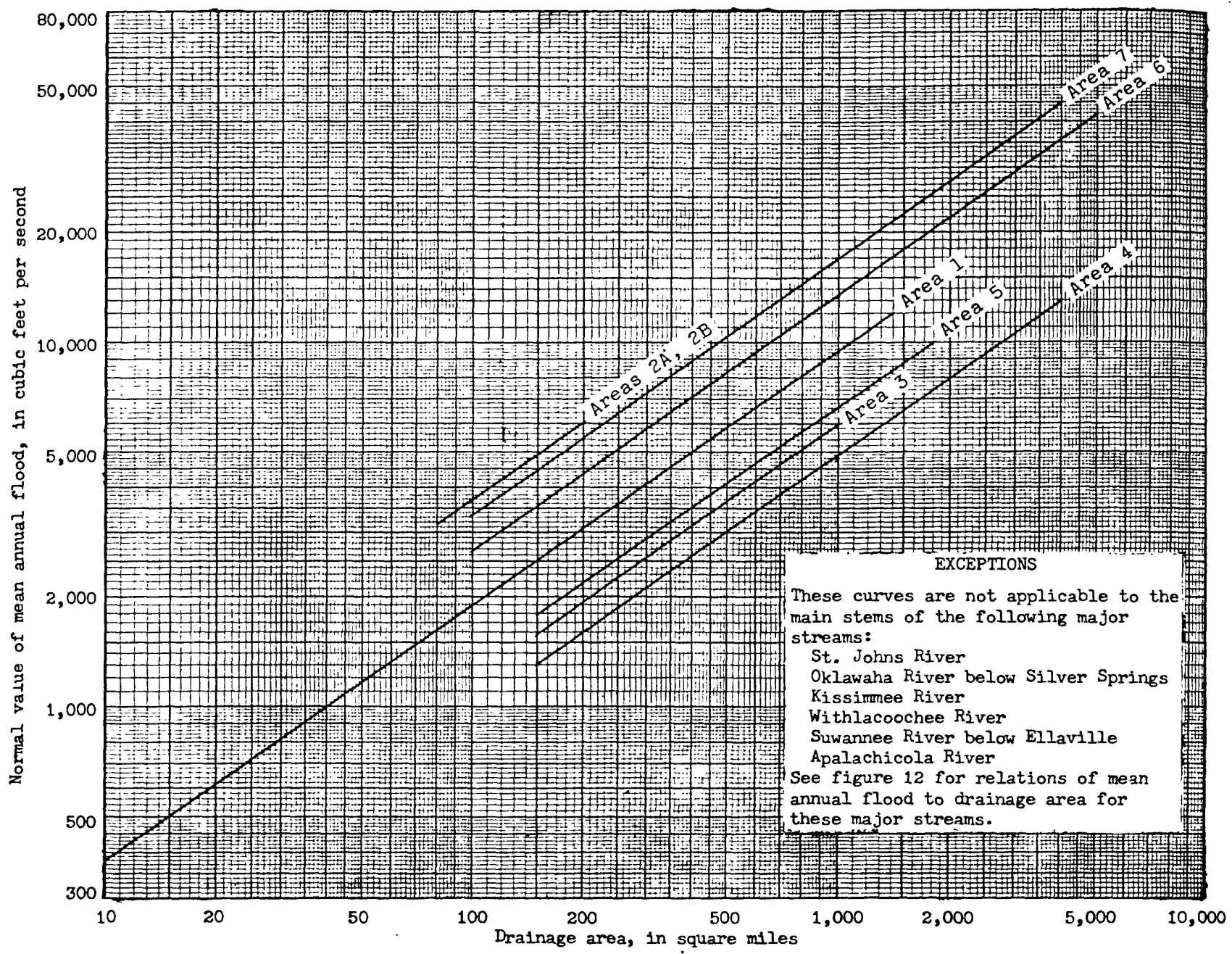

Figure 18. --Summary of relations of mean annual flood to drainage area for Florida.

The following procedure is used to estimate the flood magnitude for any selected recurrence interval within the limits of the frequency curves at a particular site on streams in Florida:

1. Determine, in square miles, the drainage area above the site.

2. Determine the total surface area of lakes connected with the drainage system and express this lake area as a percentage of the total drainage area above the site. (This step not required for the exceptions shown in figure 18).

3. Determine from plate 1 the hydrologic area in which the stream lies.

4. Determine the unadjusted mean annual flood from figure 18, using the hydrologic area determined in step 3.

5. Determine the attenuation factor from figure 10. For drainage basins devoid of lakes this factor is 1.00 .

6. Adjust the mean annual flood as obtained in step 4 for attenuation by multiplying by the attenuation factor from step 5 .

7. Determine from plate 1 the flood region in which the stream lies.

8. Determine from figure 7 or 8 the flood ratio for the desired recurrence interval, using the curve for the region determined in step 7.

9. Multiply the mean annual flood from step 6 by the flood ratio from step 8 to obtain the, desired discharge.

To estimate the flood magnitude at ungaged sites on the main stems of the large rivers that have been excepted from figure 18, obtain the mean annual flood from the relations shown in figure 12 which define the mean annual floods directly without adjustments for attenuation. Steps 2,5 , and 6 as outlined above are therefore not required for the computations when the mean annual flood is determined from figure 12. 
If desired, a complete frequency graph may be defined by plotting values determined in the above manner for a number of different recurrence intervals.

It is recommended that the above method be followed to determine magnitude and frequency at gaged sites as well. The results based on a composite frequency curve and an average relation between mean annual flood and drainage area should be more reliable than those obtained from a frequency curve based on data for the individual site alone.

\section{MAXIMUM FLOODS KNOWN}

Maximum flood stages and discharges known for Florida streams and lakes are shown in table 3 . Discharges are given both in cubic feet per second (cfs) and in cubic feet per second per square mile; stages are given as elevation in feet above the zero of the gage (gage height) and above mean sea level. Nearly all the tabulated data show the maximum occurring during the period of record at a gaging station. At some gaging stations and at several miscellaneous sites, the data for the maximum, known flood occurring before the period of record have also been included in the table, where a reliable figure was determined. The index numbers in the first column of the table correspond to those for stations described more fully in the section of this report entitled "GAGING STATION RECORDS." The stations are listed in downstream order. At stations affected by backwater, the peak gage height and peak discharge did not always occur at the same time and were shown on separate lines.

The comparison of maximum known floods in each combination of hydrologic areas and flood region $A$ with the corresponding flood of 30 -year recurrence interval is shown in figure 19. Figure $20(a, b$, and $c)$ shows the comparison of maximum known floods in region B with the corresponding 50 -year recurrence interval. These plots provide a rough means of judging the possible frequencies of the maximum flood.

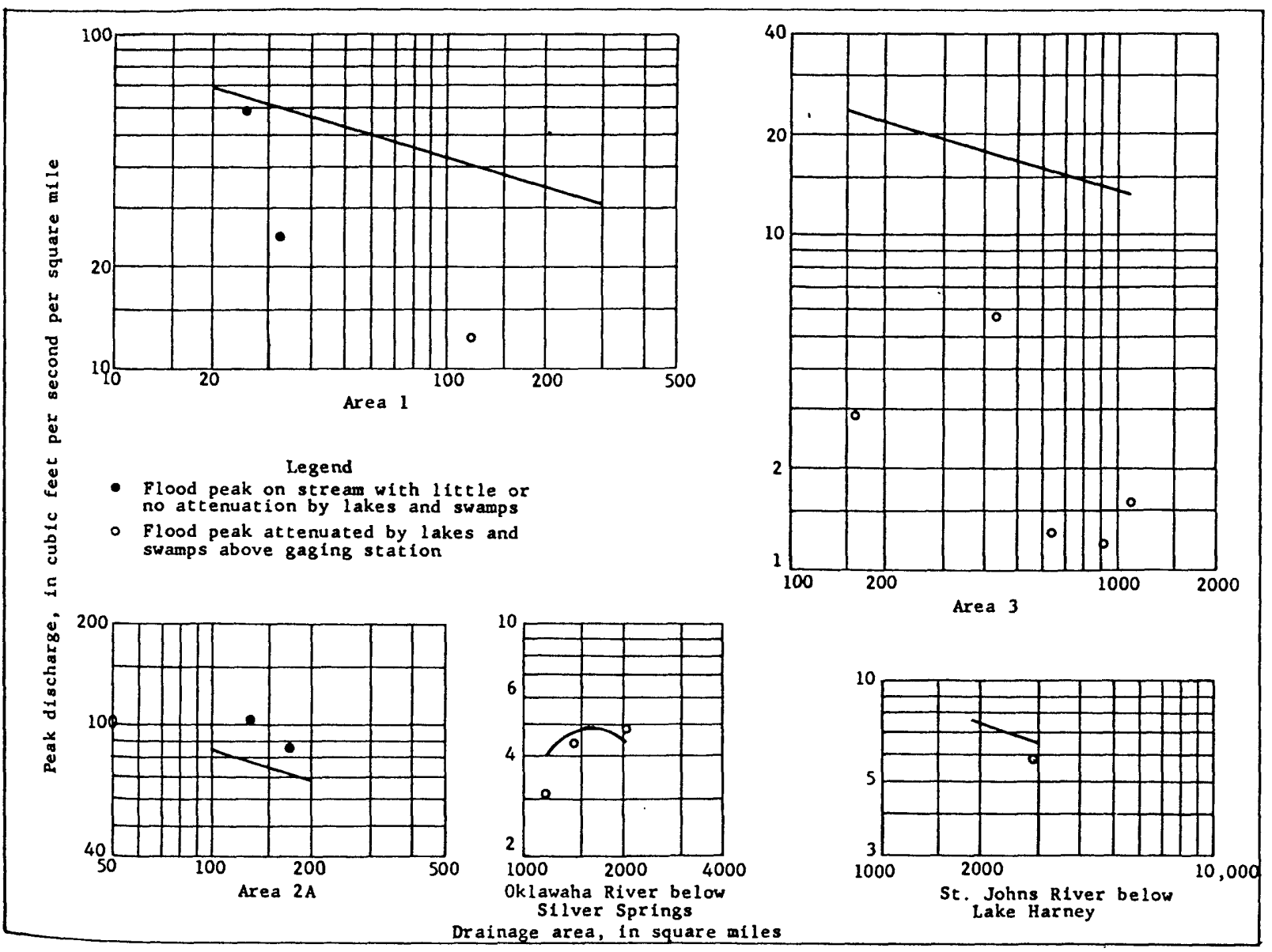

Figure 19. --Relation of maximum to 30 -year flood in region $\mathrm{A}$. 


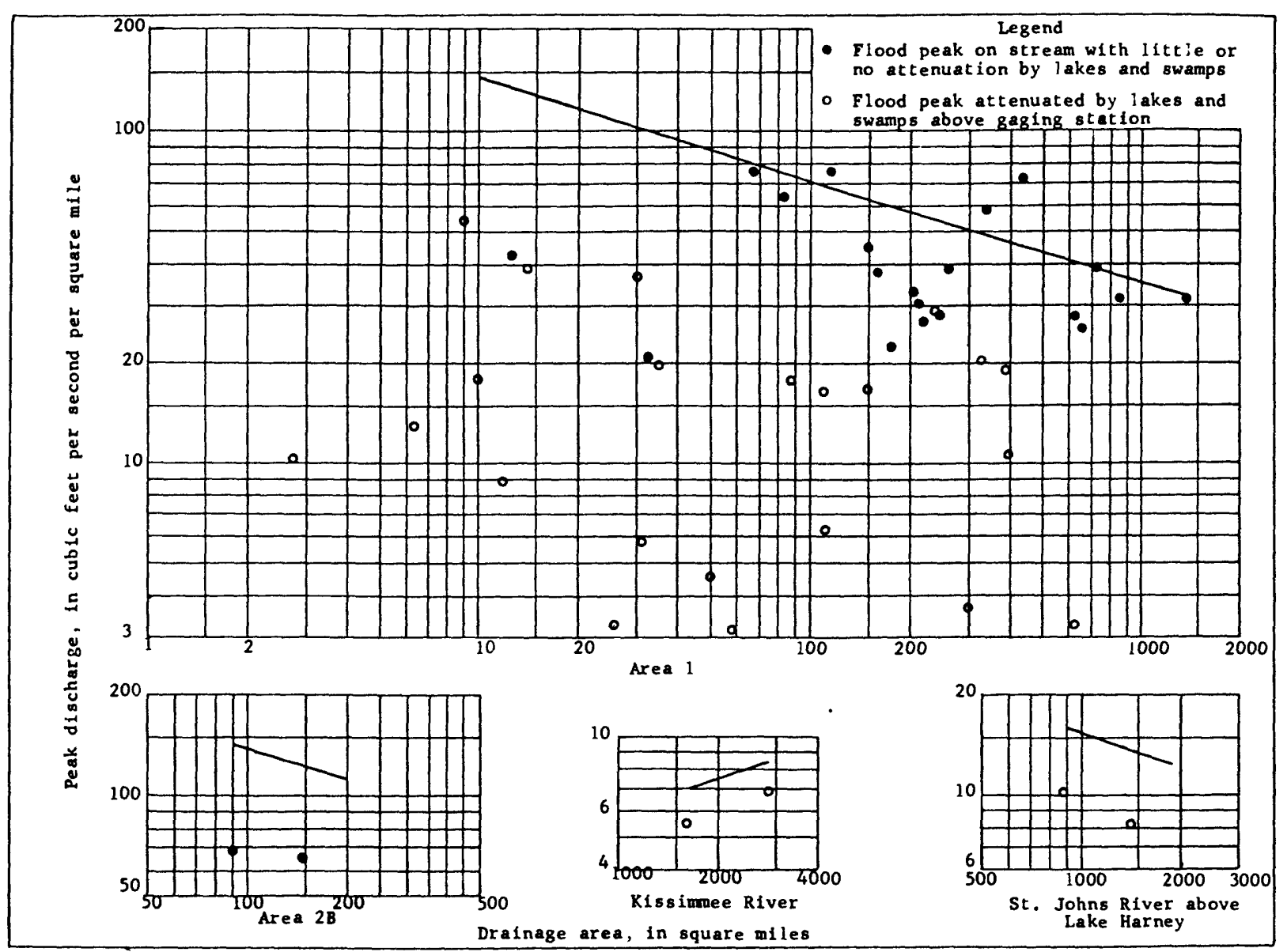

Figure 20a. --Relation of maximum to 50-year flood in region B (areas 1, 2B and Kissimmee River and St. Johns River above Lake Harney).

The attenuation of flood peaks by storage in lakes and swamps is clearly shown in the plots for several areas in figures 19 and 20. For areas 1 and 3 in figure 19 and for area 1 in figure 20a the relations for the 30or 50-year peak discharge is based on data from gaging stations relatively devoid of lakes or fram data adjusted by the attenuation factor shown in figure 10. The gaging station data plotted in these figures represent the actual maximum known floods. Thus for a tributary stream with many lakes and swamps in the drainage basins, the relations shown in these figures represent enveloping curves that will rarely be equaled. The relations and the plotted data shown for several of the larger rivers in the lake region on the Peninsula are based on existing conditions without adjustment for storage. 


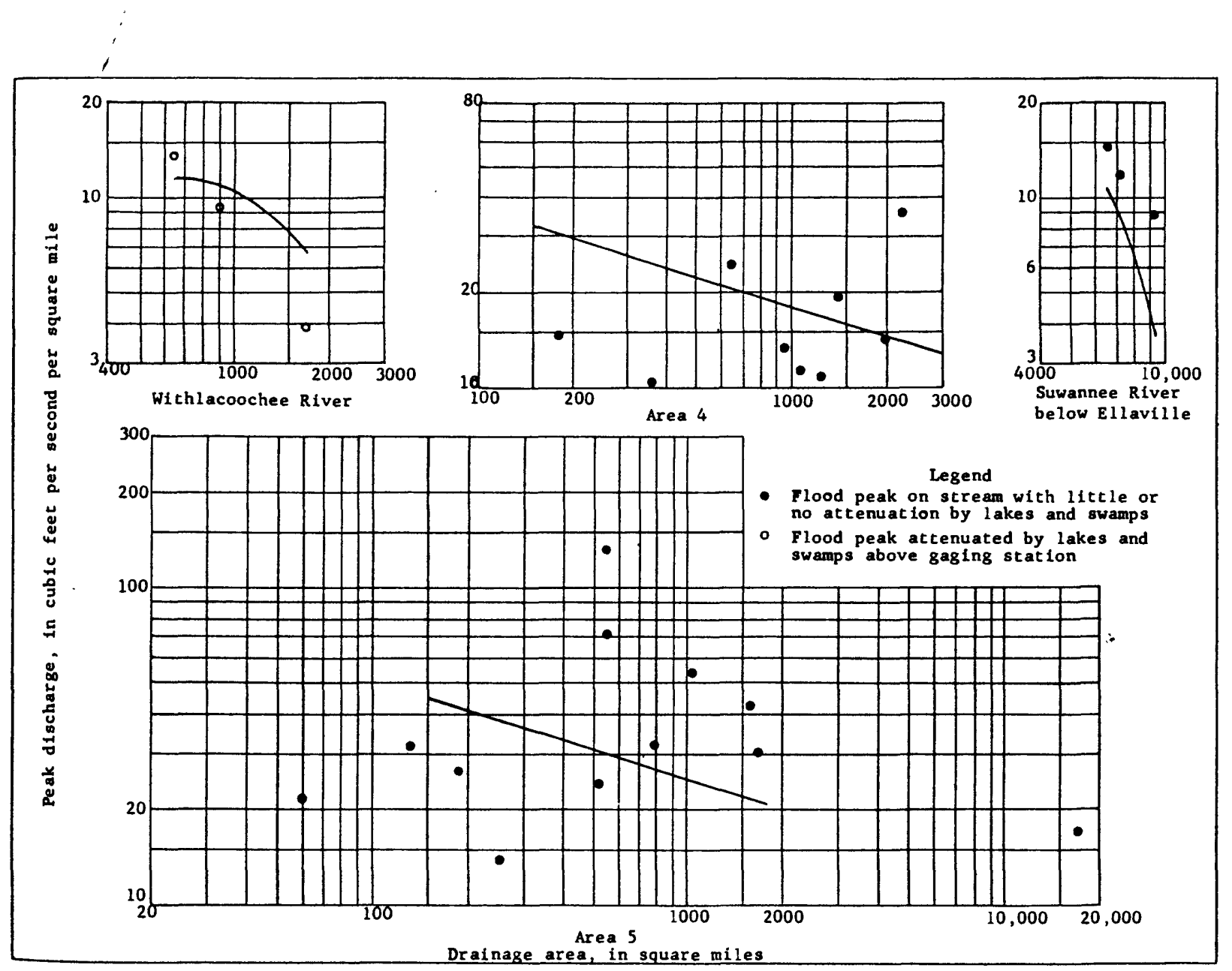

Figure 20b. --Relation of maximum to 50-year flood in region $B$ (areas 4,5 and Withlacoochee River and Suwannee River below Ellaville).

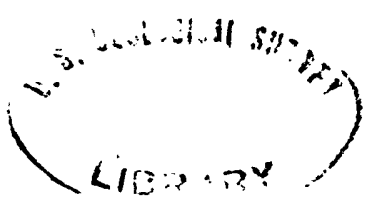




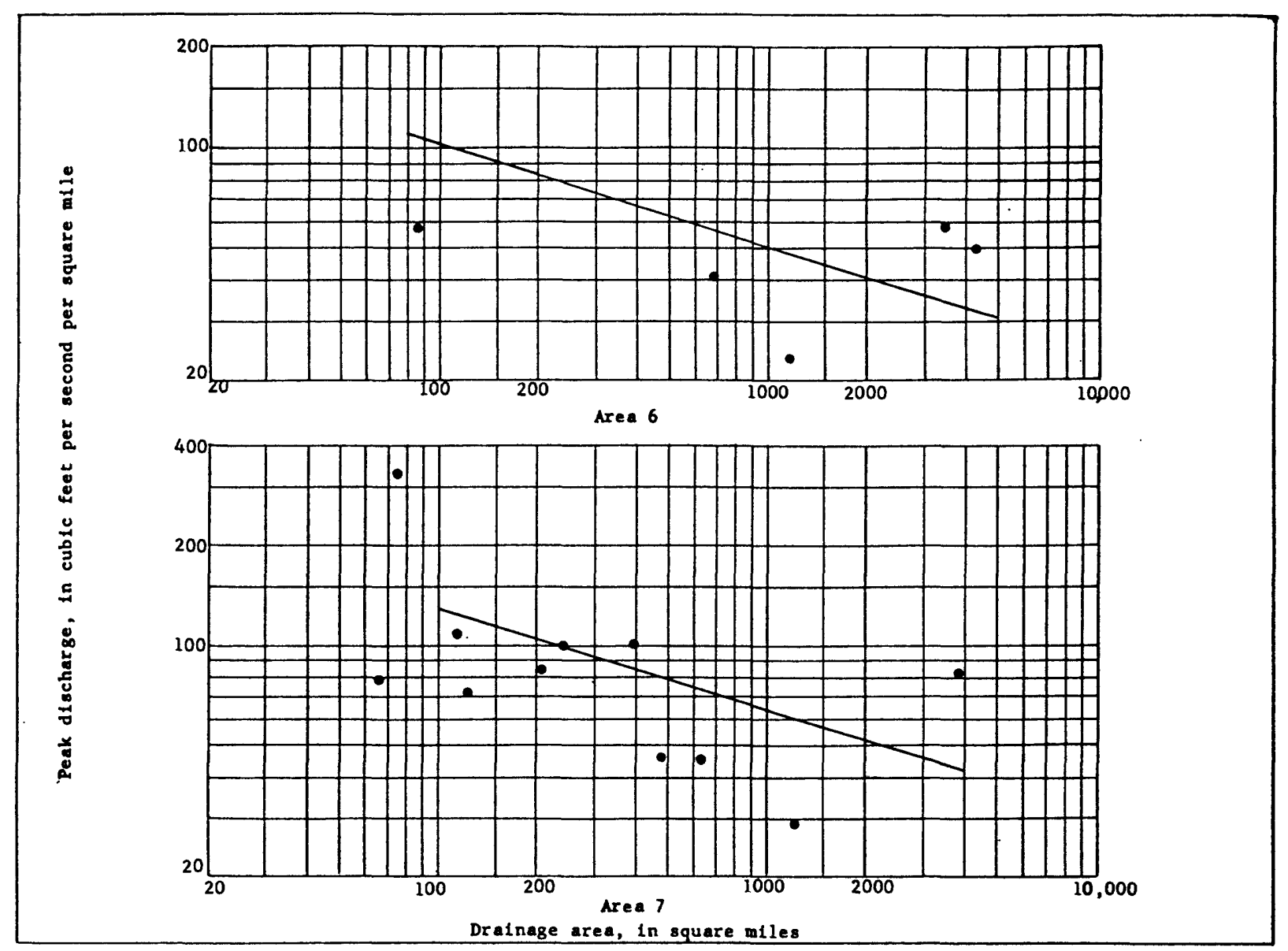

Figure 20c. --Relation of maximum to 50-year flood in region B (areas 6 and 7). 
MAXIMUM FLOODS KNOWN

Table 3. --Maximum known stages and discharges prior to 1956 in Florida

\begin{tabular}{|c|c|c|c|c|c|c|c|c|}
\hline \multirow[b]{3}{*}{ No. } & \multirow{3}{*}{$\begin{array}{l}\text { Stream or lake and place } \\
\text { of determination }\end{array}$} & \multirow{3}{*}{$\begin{array}{c}\text { Drainage } \\
\text { area } \\
\text { (sq mi) }\end{array}$} & \multirow{3}{*}{$\begin{array}{l}\text { Period } \\
\text { of } \\
\text { known } \\
\text { floods }\end{array}$} & \multicolumn{5}{|c|}{ Maximum known flood } \\
\hline & & & & \multirow[b]{2}{*}{ Date } & \multirow[b]{2}{*}{$\begin{array}{c}\text { Gage } \\
\text { height } \\
\text { (ft) }\end{array}$} & \multirow{2}{*}{$\begin{array}{c}\text { Elevation } \\
\text { above } \\
\text { msl } \\
\text { (ft) }\end{array}$} & \multicolumn{2}{|c|}{ Discharge } \\
\hline & & & & & & & Cis & $\begin{array}{c}\text { Cfs } \\
\text { per } \\
\text { (sq mi) }\end{array}$ \\
\hline & ST. MARYS RIVER BASIN & & & & & & & \\
\hline 3 & $\begin{array}{l}\text { North Prong St. Marys River at } \\
\text { Mon1ac, Ga. }\end{array}$ & 160 & $\begin{array}{l}1921-34 \\
1950-55\end{array}$ & Sept. 19,1928 & 16.7 & 109.3 & a 6,060 & 37.9 \\
\hline & $\begin{array}{l}\text { South Prong St. Marys River at glen } \\
\text { St. Mary, Fia. }\end{array}$ & 150 & $1947-55$ & September 1947 & 13.0 & 90.1 & 6,700 & 44.7 \\
\hline 4 & $\begin{array}{l}\text { St. Marys River near Macclenny, Fla--- } \\
\text { ST. JOHNS RIVER BASIN }\end{array}$ & 720 & $1,27-55$ & Sept. 25,1947 & 22.29 & 62.29 & 28,100 & 39.0 \\
\hline 5 & $\begin{array}{l}\text { St. Johns River headwaters near } \\
\text { Vero Beach, Fla. }\end{array}$ & 203 & $1942-55$ & Oct. 12,1947 & 8.81 & 27.37 & -- & -- \\
\hline 6 & $\begin{array}{l}\text { St. Johns River headwaters near } \\
\text { Kenansville, Fla. }\end{array}$ & 442 & $1942-55$ & October 1948 & 8.52 & 25.54 & -- & -- \\
\hline & Jane Green Creek near Deer Park, Fla-- & 248 & $1 j 53-55$ & Oct. 9 or 10,1953 & 8.65 & 27.20 & 6,880 & 27.7 \\
\hline 7 & St. Johns River near Melbourne, Fla--- & 874 & $1939-55$ & Oct. 12,1953 & 9.47 & 20.69 & 8,850 & 10.1 \\
\hline 8 & Lake Washington near Eau Gall1e, Fla-- & - & $1942-55$ & Oct. $12,13,1953$ & 7.68 & 20.07 & -- & -- \\
\hline 9 & Lake Polnsett near Cocoa, Fla & - & $1941-55$ & Oct. 11,1953 & 12.50 & 17.56 & -- & -- \\
\hline 10 & St. Johns R1ver near Chrlstmas, Fla--- & 1,418 & $1933-55$ & Oct. 12,1953 & 10.59 & 12.21 & 11,700 & 8.2 \\
\hline 11 & $\begin{array}{l}\text { Econlockhatchee River near Chuluota, } \\
\text { Fla. }\end{array}$ & 260 & $1935-55$ & Sept. 24,1948 & 18.09 & 20.23 & 10,000 & 38.5 \\
\hline 12 & $\begin{array}{l}\text { St. Johns River above Lake Harney, } \\
\text { near Geneva, Fla. }\end{array}$ & 1,910 & $1941-55$ & Oct. 14,1953 & 10.62 & 10.62 & -- & -- \\
\hline 13 & St. Johns R1ver at Osceola, Fla-_- & 1,950 & $1941-47$ & Sept. 21,1945 & 9.62 & 9.62 & -- & -- \\
\hline & Lake Ma1tland at winter Park, Fla-...- & - & $1945-52$ & Sept. 16,1945 & 3.04 & 67.0 & -- & -- \\
\hline 14 & Lake Jessup Outlet near Sanford, Fla-- & - & $194 \overline{1-47}$ & $\begin{array}{l}\text { July } 1930 \\
\text { Sept. } 26,1945\end{array}$ & $\overline{7.76}$ & $\begin{array}{l}8.4 \\
7.76\end{array}$ & -- & -- \\
\hline 15 & $\begin{array}{l}\text { St. Johns River above Lake Monroe, } \\
\text { near Sanford, Fla. }\end{array}$ & 2,320 & $194 \overline{1-47}$ & $\begin{array}{l}\text { October } 1924 \\
\text { Sept. } 28,1945\end{array}$ & $\overline{7.62}$ & $\begin{array}{l}8.5 \\
7.62\end{array}$ & -- & -- \\
\hline 16 & St. Johns River near Sanford, Fla & 2,420 & $\begin{array}{l}1871-1920 \\
1920-55\end{array}$ & $\begin{array}{l}1880 \\
\text { Oct. 15, } 1953\end{array}$ & 8.61 & $\begin{array}{r}13.28 \\
8.52\end{array}$ & -- & -- \\
\hline 17 & Wek1va River near Sanford, Fla-..... & - & $1935-55$ & Sept. 17, 1945 & 5.60 & -- & 2,060 & -- \\
\hline 18 & St. Johns River near DeLand, Fla----- & 2,960 & $1934-55$ & Oct. $11,12,1953$ & 7.17 & 6.06 & $b_{17,100}$ & 5.8 \\
\hline 19 & Lake Apopka at Winter Garden, Fla.... & 130 & $1936-55$ & oct. 12,1936 & -- & $c_{69.3}$ & -- & -- \\
\hline 20 & $\begin{array}{l}\text { Apopka-Beauclair Canal nr. Astatula, } \\
\text { Fla. }\end{array}$ & - & -- & Unknown & -- & 67.0 & -- & -- \\
\hline 21 & Lake Dora at Mount Dora, Fla- & - & $19 \overline{3} \overline{6}-55$ & $\begin{array}{l}\text { September } 1926 \\
\text { Jan. 4-7, } 1954\end{array}$ & -- & $\begin{array}{l}65.8 \\
65.40\end{array}$ & -- & -- \\
\hline 22 & Dead River near Tavares, Fla & - & $19 \overline{43-55}$ & $\begin{array}{l}1926 \\
\text { Jan. } 1-4,1954\end{array}$ & $\overline{5.24}$ & $\begin{array}{l}66.1 \\
64.54\end{array}$ & -- & -- \\
\hline 23 & Lake Minnehaha at Clermont, Fla-..-- & - & & Oct. $9,10,1953$ & 7.52 & 98.84 & -- & -- \\
\hline & Palatlakaha Creek at Groveland, Fla--- & - & $1945-55$ & Sept. 17, 1945 & -- & 97.43 & -- & -- \\
\hline 24 & Palatlakaha Creek near Mascotte, Fla-- & 160 & $1945-55$ & $\begin{array}{l}\text { Oct. } 4,5,1945 \\
\text { Oct. } 11,12,1953\end{array}$ & $\overline{7.12}$ & $9 \overline{6.66}$ & -- & 2.9 \\
\hline 25 & Haines Creek at'L1sbon, Fla- & 640 & $19 \overline{43-55}$ & $\begin{array}{l}1926 \\
\text { Jan. } 3-5,1954\end{array}$ & 4.24 & $\begin{array}{l}65.6 \\
63.46\end{array}$ & $-\overline{819}$ & $\overline{1.3}$ \\
\hline 26 & Lake We $1 \mathrm{r}$ at Oklawaha, Fla & - & $1936-55$ & January 1938 & -- & $c_{59.6}$ & - & -- \\
\hline 27 & Oklawaha R1ver at Moss Bluff, Fla-... & 910 & $1944-55$ & Jan. 11, 12, 1954 & 49.49 & 49.49 & 1,060 & 1.2 \\
\hline 28 & Oklawaha R1ver near Ocala, Fla & 1,100 & $1930-55$ & $\begin{array}{l}\text { Sept. 6, } 1933 \\
\text { June } 15,1934\end{array}$ & 5.52 & 42.04 & $\overline{1,810}$ & $\overline{1.6}$ \\
\hline 29 & S1lver Springs near Ocala, Fla & - & $1933-55$ & $\begin{array}{l}\text { Sept. } 6,1933 \\
\text { Nov. } 2-4,1950\end{array}$ & 5.50 & $\begin{array}{l}44.46 \\
--\end{array}$ & $\overline{1,150}$ & -- \\
\hline 30 & Oklawaha River near Conner, Fla_...- & 1,180 & $1930-46$ & Sept. 6, 1933 & 9.14 & 40.93 & 3,700 & 3.1 \\
\hline 31 & Oklawaha R1ver at Eureka, Fla & 1,420 & $1930-52$ & Sept. 7, 1933 & 11.00 & 26.44 & 6,260 & 4.4 \\
\hline 32 & Newnans Lake near Galnesville, Pla-.-- & - & $1936-52$ & Mar. 12,1948 & 7.88 & 71.21 & -- & -- \\
\hline
\end{tabular}


Table 3. --Maximum known stages and discharges prior to 1956 in Florida--continued

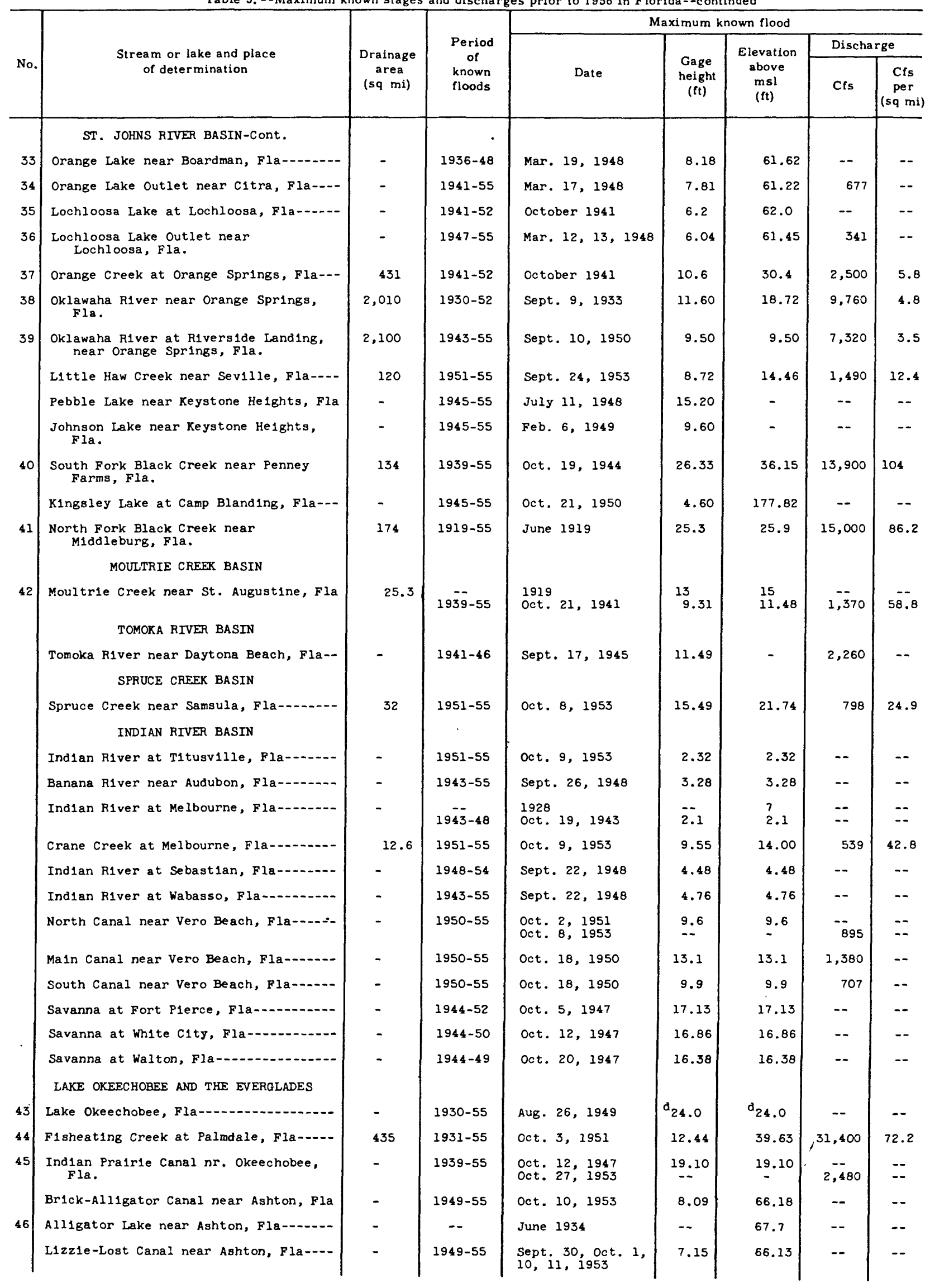


MAXIMUM FLOODS KNOWN

Table 3. --Maximum known stages and discharges prior to 1956 in Florida--continued

\begin{tabular}{|c|c|c|c|c|c|c|c|c|}
\hline \multirow{3}{*}{ No. } & \multirow{3}{*}{$\begin{array}{l}\text { Stream or lake and place } \\
\text { of determination }\end{array}$} & \multirow{3}{*}{$\begin{array}{l}\text { Drainage } \\
\text { area } \\
\text { (sq mi) }\end{array}$} & \multirow{3}{*}{$\begin{array}{l}\text { Period } \\
\text { of } \\
\text { known } \\
\text { floods }\end{array}$} & \multicolumn{5}{|c|}{ Maximum known flood } \\
\hline & & & & \multirow[b]{2}{*}{ Date } & \multirow[b]{2}{*}{$\begin{array}{l}\text { Gage } \\
\text { height } \\
\text { (ft) }\end{array}$} & \multirow{2}{*}{$\begin{array}{l}\text { Elevation } \\
\text { above } \\
\text { msl } \\
\text { (ft) }\end{array}$} & \multicolumn{2}{|c|}{ Discharge } \\
\hline & & & & & & & Cis & $\begin{array}{c}\text { Cefs } \\
\text { per } \\
(\mathrm{sq} \mathrm{mi})\end{array}$ \\
\hline & LAKE OKEECHOBEE AND THE EVERGLADES-Cont. & & & & & & & \\
\hline & $\begin{array}{l}\text { Myrtle-Mary Jane Canal near } \\
\text { Narcoossee, Pla. }\end{array}$ & 111 & $1949-55$ & Oct. 1,1953 & 7.67 & 64.75 & 703 & 6.3 \\
\hline & Mary Jane Lake near Narcoossee, Fla--- & - & $1949-55$ & $\begin{array}{l}\text { Oct. } 1,10-12, \\
1953\end{array}$ & 7.33 & 63.99 & -- & -- \\
\hline \multirow[t]{2}{*}{47} & Hart Lake near Narcoossee, Pla-- & - & $1941-55$ & Sept. 20,1945 & 9.13 & 64.87 & -- & -- \\
\hline & Lake Conway near P1necastle, Pla-.--- & - & $19 \overline{2-55}$ & $\begin{array}{l}\text { Unknown } \\
\text { Dec. 5-8, } 1953\end{array}$ & 19.20 & $\begin{array}{l}\text { e. } \\
88.4\end{array}$ & -- & -- \\
\hline \multirow[t]{2}{*}{48} & $\begin{array}{l}\text { East Tohopekal1ga Lake at St. Cloud, } \\
\text { Fla. }\end{array}$ & - & $19 \overline{1-55}$ & $\begin{array}{l}\text { July } 1930 \\
\text { Oct. 12, 13, } 1953\end{array}$ & $1 \overline{0.63}$ & $\begin{array}{l}62.17 \\
62.01\end{array}$ & -- & -- \\
\hline & $\begin{array}{l}\text { East Tohopekal1ga-Tohopekal1ga Canal } \\
\text { near St. Cloud, Fla. }\end{array}$ & 300 & $1950-55$ & $\begin{array}{l}\text { Sept. } 19,1945 \\
\text { Oct. } 13,1953\end{array}$ & $\overline{9.48}$ & $6 \overline{1.80}$ & $\begin{array}{r}f_{1}, 140 \\
1,110\end{array}$ & $\overline{3.7}$ \\
\hline \multirow[t]{2}{*}{49} & Lake Tohopekal1ga at K1ss1mmee, Fla--- & - & $1942-55$ & oct. $10,11,1953$ & 58.62 & 58.62 & -- & -- \\
\hline & $\begin{array}{l}\text { Tohopekallga-Cypress Canal near } \\
\text { St. Cloud, Pla. }\end{array}$ & - & $1942-55$ & Oct. 9,1953 & 10.25 & 59.01 & 2,650 & -- \\
\hline \multirow[t]{3}{*}{50} & Cypress Lake near St. Cloud, Fla-..-- & - & $1942-55$ & Oct. $12,13,1953$ & 8.38 & 57.16 & -- & -- \\
\hline & Lake Gentry near St. Cloud, Fla-.--- & $-{ }^{\cdot}$ & $1949-55$ & Oct. 10,1953 & 5.71 & 63.13 & -- & -- \\
\hline & Canoe Creek near St. Cloud, Pla-- & 86.5 & $1949-55$ & $\begin{array}{l}1935 \\
\text { Oct. } 19,1950\end{array}$ & $1 \overline{11.4}$ & $\begin{array}{l}61.7 \\
59.6\end{array}$ & $1,-\overline{5} 50$ & 17.9 \\
\hline 51 & Lake Butler at Windermere, Pla & - & $19 \overline{3}-35$ & $\begin{array}{l}\text { September } 1926 \\
\text { Sept. } 17,1945\end{array}$ & 4.88 & $\begin{array}{l}101.30 \\
101.28\end{array}$ & -- & -- \\
\hline 52 & Cypress Creek at Vineland, Fla..... & 31.0 & $1945-55$ & Sept. 16,1945 & 3.83 & 100.03 & 181 & 5.8 \\
\hline 53 & Reedy Creek near Loughman, Fla.....- & - & $1939-55$ & $\begin{array}{l}\text { Oct. } 22,23,1944 ; \\
\text { Sept. } 20,21,1947 \\
\text { Aug. } 30,1953\end{array}$ & $\overline{4.08}$ & 68.57 & 530 & -- \\
\hline 54 & Lake Hatchineha near Haines C1ty, Pla- & - & $19 \overline{42}-55$ & $\begin{array}{l}1934 \\
\text { Oct. } 14,15,19 \\
1953\end{array}$ & $\overline{9.61}$ & $\begin{array}{l}57.0 \\
56.84\end{array}$ & -- & -- \\
\hline \multirow[t]{3}{*}{55} & Catf1sh Creek near Lake Wales, Fla---- & 58.9 & $1947-55$ & oct. 9, 1953 & 5.81 & 78.51 & 191 & 3.2 \\
\hline & $\begin{array}{l}\text { Hatchineha-K1ssimmee Canal near } \\
\text { Lake Wales, Fla. }\end{array}$ & - & $1949-55$ & oct. 1,1953 & -- & - & 2,820 & -- \\
\hline & $\begin{array}{l}\text { Cypress-Kissimmee Canal near Lake } \\
\text { Wales, Fla. }\end{array}$ & - & $1950-55$ & Oct. 16,1953 & -- & - & 3,230 & -- \\
\hline 56 & Lake K1ss1mmee near Lake Wales, Pla--- & - & $1942-55$ & September 1947 & 9.77 & 56.71 & -- & -- \\
\hline 57 & $\begin{array}{l}\text { Weohyakapka-Rosalle Canal near } \\
\text { Lake Wales, Fla. }\end{array}$ & - & -- & September 1933 & -- & 62.8 & -- & -- \\
\hline 58 & $\begin{array}{l}\text { K1ssimmee R1ver below Lake K1ssimmee, } \\
\text { Fla. }\end{array}$ & 1,609 & $1930-55$ & $\begin{array}{l}\text { oct. } 5 \text { or } 6,1948 \\
\text { oct. } 9,1953\end{array}$ & $\overline{13.16}$ & $5 \overline{6} .64$ & 8,820 & $\begin{array}{l}5.5 \\
--\end{array}$ \\
\hline \multirow[t]{3}{*}{59} & K1ss1mmee R1ver at Port K1ssimmee, Fla & - & $1941-55$ & oct. 11,1953 & 12.89 & 50.92 & -- & -- \\
\hline & Crooked Lake near Babson Park, Fla--.- & & $1945-55$ & Oct. 8,1948 & 8.30 & 124.01 & -- & -- \\
\hline & Lake Clinch at Frostproof, Fla-- & - & $1947-55$ & Oct. 10,1948 & 10.21 & 110.21 & -- & -- \\
\hline 60 & Reedy Lake outlet near Frostproof, Fla & 62.2 & $1946-55$ & oct. 5,1948 & 4.37 & 80.42 & 166 & 2.7 \\
\hline \multirow[t]{3}{*}{61} & Lake Arbuckle near Avon Park, Fla--.-- & - & $19 \overline{4}-55$ & $\begin{array}{l}1926,1928 \\
\text { Sept. } 24,1948\end{array}$ & $\overline{6.7}$ & $\begin{array}{l}58.7 \\
58.2\end{array}$ & $\overline{--}$ & -- \\
\hline & Lake Lotela at Avon Park, Fla & - & $1950-55$ & Sept. 30,1953 & 8.92 & 108.85 & -- & - \\
\hline & Lake Letta at Avon Park, Fla & - & $1951-55$ & Oct. 7,1953 & 5.07 & 101.38 & -- & -- \\
\hline \multirow[t]{5}{*}{62} & $\begin{array}{l}\text { Arbuckle Creek near DeSota C1ty, Fla-- } \\
\text { Rex Beach Lake at Sebring, Pla- }\end{array}$ & $\begin{array}{l}385 \\
-\end{array}$ & $1939-55$ & $\begin{array}{l}\text { Sept. } 23,1948 \\
1941 \\
\text { Sept. } 19,1947\end{array}$ & $\begin{array}{l}8.71 \\
\overline{7.45}\end{array}$ & $\begin{array}{l}44.22 \\
104.1 \\
103.76\end{array}$ & $\begin{array}{l}7,380 \\
-- \\
--\end{array}$ & $\begin{array}{c}19.2 \\
-- \\
--\end{array}$ \\
\hline & Lake Joseph1ne near DeSoto C1ty, Fla-- & - & $1946-55$ & Sept. 26,1948 & 11.1 & 76.8 & -- & -- \\
\hline & Lake Plac1d near Lake Plac1d, Pla-.--- & - & $1945-55$ & Sept. 25,1948 & 15.98 & 95.64 & -- & -- \\
\hline & M1rror Lake near Lake Plac1d, Pla-..-- & - & $1951-55$ & Oct. 10,1953 & 10.52 & 94.93 & -- & -- \\
\hline & $\begin{array}{l}\text { Lake June-1n-Winter near Lake Plac1d, } \\
\text { Pla. }\end{array}$ & - & $1945-55$ & October 1950 & 12.2 & 77.6 & -- & -- \\
\hline
\end{tabular}


Table 3, --Maximum known stages and discharges prior to 1956 in Florida--continued

\begin{tabular}{|c|c|c|c|c|c|c|c|c|}
\hline \multirow[b]{3}{*}{ No. } & \multirow{3}{*}{$\begin{array}{l}\text { Stream or lake and place } \\
\text { of determination }\end{array}$} & \multirow{3}{*}{$\begin{array}{c}\text { Drainage } \\
\text { area } \\
\text { (sq mi) }\end{array}$} & \multirow{3}{*}{$\begin{array}{l}\text { Period } \\
\text { of } \\
\text { known } \\
\text { noods }\end{array}$} & \multicolumn{5}{|c|}{ Maximum known flood } \\
\hline & & & & \multirow[b]{2}{*}{ Date } & \multirow[b]{2}{*}{$\begin{array}{c}\text { Gage } \\
\text { height } \\
\text { (ft) }\end{array}$} & \multirow{2}{*}{$\begin{array}{c}\text { Elevation } \\
\text { above } \\
\text { msl } \\
\text { (ft) }\end{array}$} & \multicolumn{2}{|c|}{ Discharge } \\
\hline & & & & & & & $\mathrm{Crs}$ & $\begin{array}{c}\text { Cfs } \\
\text { per } \\
\text { (sq mi) }\end{array}$ \\
\hline \multirow{5}{*}{63} & LAKE OKEECHOBEE AND THE EVERGLADES-Cont. & & & & & & & \\
\hline & Joseph1ne Creek near DeSoto C1ty, Fla- & 109 & $1946-55$ & Sept. 23,1948 & 11.56 & 64.55 & 1,780 & 16.3 \\
\hline & Grassy Lake near Lake Plac1d, Fla----- & - & $195 \lambda-55$ & Sept. 19,1953 & 25.36 & 94.26 & -- & -- \\
\hline & Lake Huntley at Lake Plac1d, Fla-_... & - & $1951-55$ & oct. 13,1953 & 15.50 & 84.42 & -- & -- \\
\hline & Lake Clay at Lake Plac1d, Fla_..... & - & $1951-55$ & oct. 14,1953 & 10.50 & 79.44 & -- & -- \\
\hline 64 & Lake Istokpoga near DeSoto C1ty, Fla-- & - & $1936-55$ & September 1945 & -- & 43.22 & -- & -- \\
\hline 65 & Istokpoga Canal near Cornwell, Fla---- & 624 & $1933-55$ & $\begin{array}{l}\text { Sept. } 22,1948 \\
\text { oct. } 12,1953\end{array}$ & $\overline{11.41}$ & $4 \overline{1.12}$ & 2,040 & 3.3 \\
\hline 66 & K1ss1mmee R1ver near Cornwell, Fla--.- & 2,703 & $1928-55$ & oct. $12,13,1953$ & 21.48 & 36.12 & -- & -- \\
\hline 67 & K1ssimmee R1ver near Okeechobee, Fla-- & 2,886 & $1928-55$ & August 2928 & 30.3 & 28.9 & 20,000 & 6.9 \\
\hline 68 & $\begin{array}{l}\text { St. Lucle Canal at Lake Okeechobee, } \\
\text { Fla. }\end{array}$ & - & $1931-51$ & Nov. $2,6,2951$ & -- & - & 87,470 & -- \\
\hline \multirow[t]{2}{*}{69} & $\begin{array}{l}\text { West Palm Beach. Canal at Canal Polnt, } \\
\text { Fla. }\end{array}$ & - & $2940-55$ & $\begin{array}{l}\text { June } 15,1942 \\
\text { Oct. } 23,1947 \\
\text { Mar. } 18,1948\end{array}$ & 18.54 & $1 \overline{8} .54$ & $\begin{array}{c}\mathrm{gh}_{1}, 760 \\
\mathrm{~g} 817\end{array}$ & $\begin{array}{l}-- \\
-- \\
--\end{array}$ \\
\hline & $\begin{array}{l}\text { West Palm Beach Canal at 20-mile } \\
\text { bend, Fla. }\end{array}$ & - & $1947-50$ & oct. $16,17,1947$ & 27,48 & 17.48 & -- & -- \\
\hline 70 & $\begin{array}{l}\text { West Palm Beach Canal at West Palm } \\
\text { Beach, Fla. }\end{array}$ & - & -- & oct. $23,24,1924$ & ${ }^{1} 13.20$ & ${ }^{1} 13.20$ & 8,570 & -- \\
\hline 72 & H1llsboro Canal at Belle Glade, Fla--- & - & $1940-55$ & $\begin{array}{l}\text { Feb. } 14,1940 \\
\text { Sept. 9, 1940 } \\
\text { Oct. 15, 16, } 1947\end{array}$ & $\overline{--}$ & $\overline{-}$ & $\begin{array}{r}\text { 8481 } \\
\text { 8h289 } \\
--\end{array}$ & $\begin{array}{l}-- \\
--\end{array}$ \\
\hline \multirow[t]{4}{*}{72} & $\begin{array}{l}\text { H1lisboro Canal at Shawano Plantation, } \\
\text { Fla. }\end{array}$ & - & $1929-52$ & oct. $12,13,1947$ & $17 . \dot{81}$ & 26.37 & -- & - \\
\hline & $\begin{array}{l}\text { H1lisboro Canal at Indian Run near } \\
\text { Deerfleld Beach, Fla. }\end{array}$ & - & $1947-53$ & Oct. 12,1947 & 15.54 & 15.54 & -- & -- \\
\hline & $\begin{array}{l}\text { Indian Run at H1dlsboro Canal near } \\
\text { Deerfleld Beach, Fla. }\end{array}$ & - & $2947-55$ & oct. 12,1947 & 15.95 & 15.95 & -- & -- \\
\hline & $\begin{array}{l}\text { H1llsboro Canal at Range Line Road } \\
\text { near Deerfield Beach, Fla. }\end{array}$ & - & $1947-52$ & Oct. 12,1947 & 14.70 & 14.70 & -- & -- \\
\hline 73 & $\begin{array}{l}\text { H1llsboro Canal near Deerfleld Beach, } \\
\text { Fla. }\end{array}$ & - & $2939-52$ & $\begin{array}{l}\text { oct. } 17,2944 \\
\text { oct. } 12,1947\end{array}$ & ${ }^{1} 12.10$ & ${ }^{12.10}$ & $8_{3,490}$ & -- \\
\hline 74 & $\begin{array}{l}\text { North New River Canal at South Bay, } \\
\text { Fla. }\end{array}$ & - & $19 \overline{-}-5-55$ & $\begin{array}{l}\text { July } 27,28,1926 \\
\text { June 10, } 1942 \\
\text { Sept. 30, } 1947\end{array}$ & $\begin{array}{l}20.56 \\
-- \\
--\end{array}$ & $\begin{array}{l}20.56 \\
- \\
-\end{array}$ & $\begin{array}{l}\mathrm{gh}^{--} 404 \\
\mathrm{~B}_{1}, 040\end{array}$ & $\begin{array}{l}-- \\
--\end{array}$ \\
\hline 75 & $\begin{array}{l}\text { North New River Canal at 26-m1le bend, } \\
\text { near Fort Lauderdale, Fla. }\end{array}$ & - & $1942-52$ & Oct. 12,1947 & ${ }^{1} 12.97$ & 112.97 & -- & -- \\
\hline \multirow[t]{6}{*}{76} & $\begin{array}{l}\text { North New River Canad near Fort } \\
\text { Lauderdale, Fla. }\end{array}$ & - & $19 \overline{40-55}$ & $\begin{array}{l}\text { oct. } 15,1929 \\
\text { oct. } 17,1947 \\
\text { Nov. } 19,1947\end{array}$ & 120.83 & ${ }_{-}^{10.03}$ & $\begin{array}{c}5,400 \\
B_{3,280}\end{array}$ & $\overline{--}$ \\
\hline & South New River Canal at Davie, Fla--- & - & $2947-55$ & oct. 21,2947 & 17.78 & 17.78 & - & -- \\
\hline & $\begin{array}{l}\text { Snake Creek Canal at North Miam1 } \\
\text { Beach, Fla. }\end{array}$ & - & $2947-55$ & Sept. 22,1948 & 14.24 & 14.24 & -- & -- \\
\hline & B1 scayne Canal at North M1am1, Fla---- & - & $2946-55$ & Oct. 15,1947 & 15.43 & 15.43 & -- & -- \\
\hline & $\begin{array}{l}\text { Little River Canal at N.W. } 7 \text { th Ave., } \\
\text { Mlam1, Fla. }\end{array}$ & - & $1946-55$ & oct. 15,1947 & 14.68 & 14.68 & -- & -- \\
\hline & $\begin{array}{l}\text { Miami Canal above broken dam, near } \\
\text { M1am1, Fla. }\end{array}$ & - & $1941-55$ & oct. 12,1947 & 10.05 & 9.82 & -- & -- \\
\hline \multirow[t]{2}{*}{77} & $\begin{array}{l}\text { M1am1 Canal at Pennsuco, near M1am1, } \\
\text { Fla. }\end{array}$ & - & $2926-55$ & oct. 12,1947 & 9.58 & 9.00 & -- & -- \\
\hline & $\begin{array}{l}\text { Miam1 Canal Russian Colony Canal, } \\
\text { near Hialeah, Fla. }\end{array}$ & - & $194 \lambda-55$ & oct. 12,2947 & 8.8 & 8.7 & -- & -- \\
\hline \multirow[t]{2}{*}{78} & $\begin{array}{l}\text { Mlami Canal at water plant, Hialeah, } \\
\text { Fla. }\end{array}$ & - & $1940-55$ & Oct. 15,1947 & 7.34 & 7.33 & 84,170 & -- \\
\hline & $\begin{array}{l}\text { M1ami Canal at N.W. } 27 \text { th Ave., } \\
\text { M1am1, Fla. }\end{array}$ & - & $1945-55$ & oct. 11,1947 & 4.57 & 4.57 & -- & -- \\
\hline 79 & $\begin{array}{l}\text { Tamlami Canal outlets, M1am1 to } \\
\text { Monroe, Fla. }\end{array}$ & - & $1939-55$ & oct. 12,1947 & -- & - & $8_{17,100}$ & -- \\
\hline
\end{tabular}


MAXIMUM FLOODS KNOWN

Table 3. --Maximum known stages and discharges prior to 1956 in Florida--continued

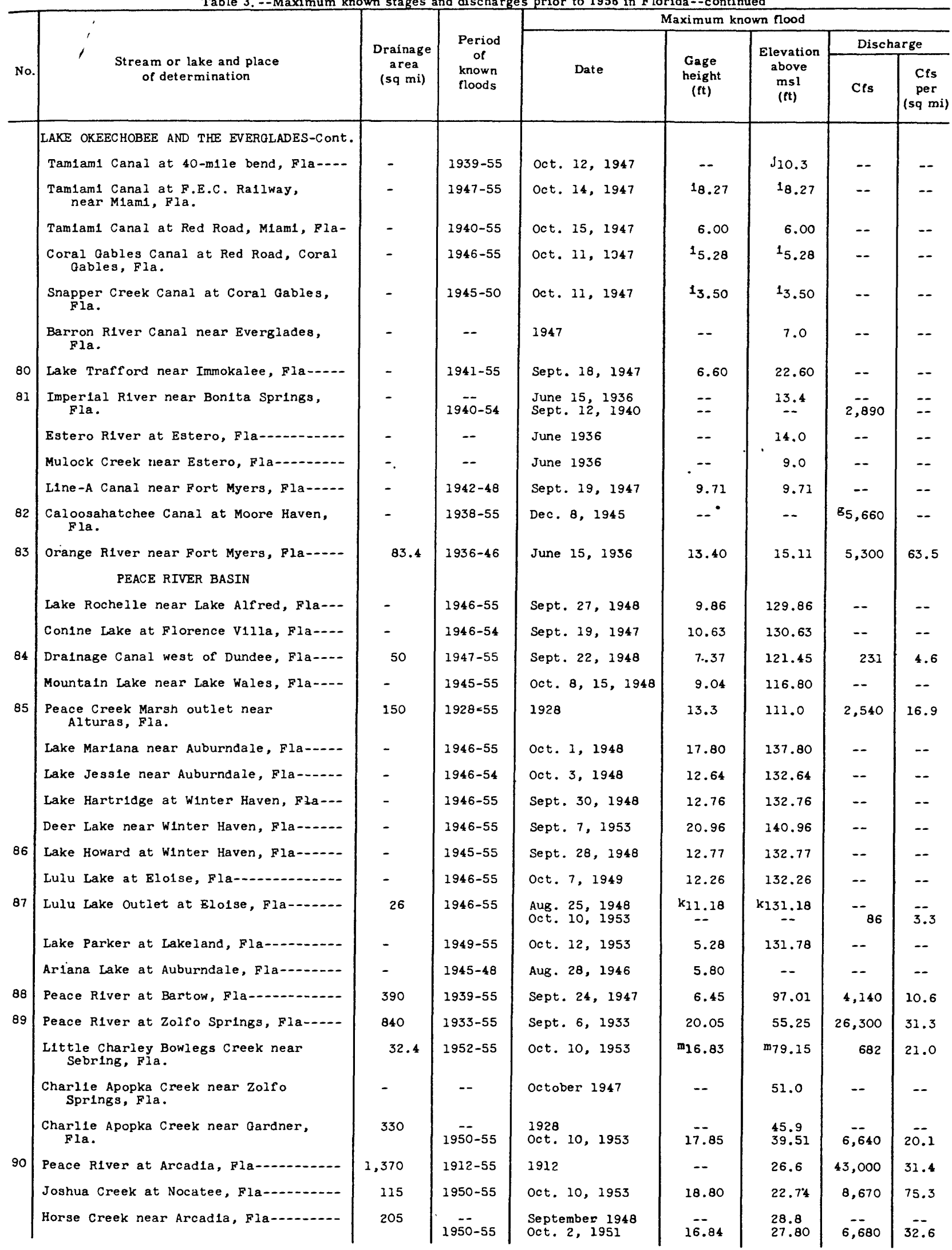


Table 3. --Maximum known stages and discharges prior to 1956 in Florida--continued




MAXIMUM FLOODS KNOWN

Table 3. --Maximum known stages and discharges prior to 1956 in F:orida--continued

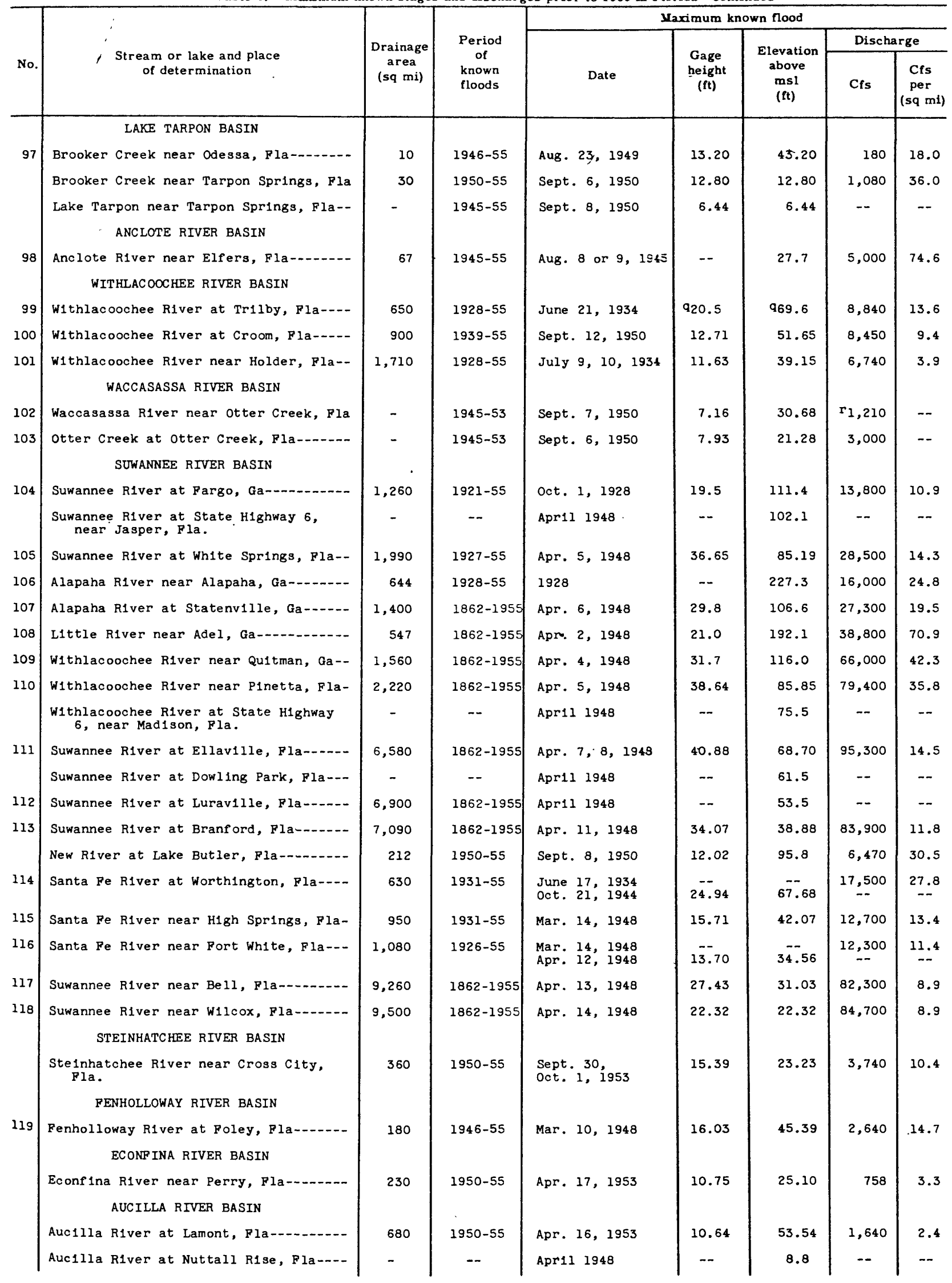


Table 3. - -Maximum known stages and discharges prior to 1956 in Florida--continued




Table 3. --Maximum known stages and discharges prior to 1956 in Florida--continued

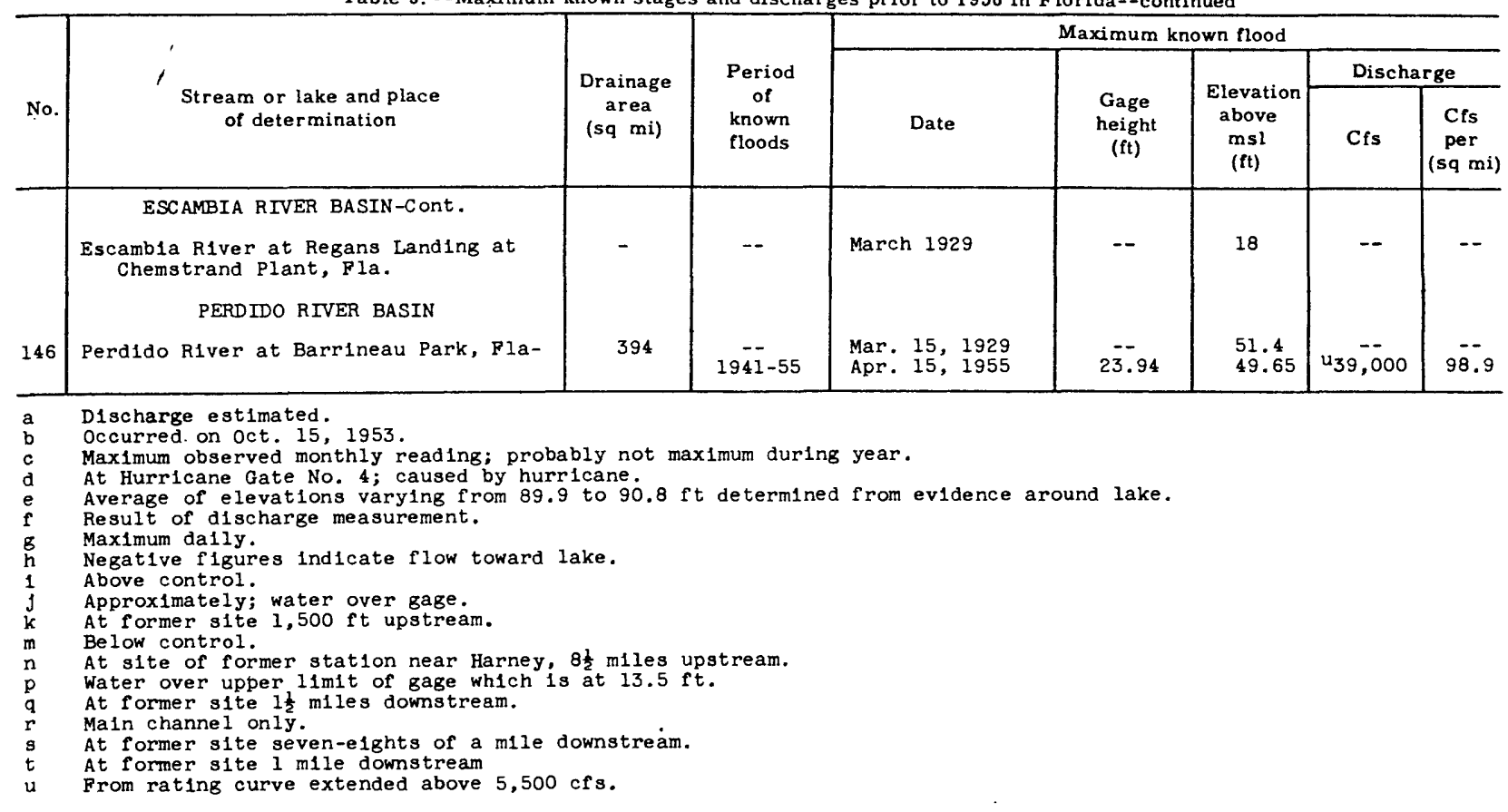

\section{GAGING STATION $\cdot$ RECORDS}

Annual peak gage heights and discharges at gaging stations in Florida are tabulated in this section. These data provide the basis for the study of flood frequency and magnitude presented on previous pages of this report.

Gage heights represent the water level in feet above an arbitrary datum which is referenced to local benchmarks at the gaging station. The elevation of this arbitrary datum above mean sea level is also given in the station description. Changes in datum are noted in the station description. Where a change in datum has been made and the gage has remained at essentially the same site, all gage heights have been converted to the datum of the present gage in the tabulation of annual flood peaks. Significant changes in site are denoted by a full line between two items in the tabulation.

Gage heights listed are the maximum gage height during each calendar year from the beginning of the record to Dec. 31, 1953. The calendar year was chosen rather than the water year as the base period for listing of annual poaks as it more nearly coincides with the climatic year in Florida than does the water year.

The gage heights were obtained generally from water-stage recorder charts or from gage readings by an observer. Many of the gage heights shown for major floods outside the period of record were obtained by leveling to floodmarks pointed out by local residents.

Peak discharges are computed from peak gage heights through the medium of a stage-discharge relation, except for those streams where this relation is affected by backwater. Above the range defined by current-meter measurements the stage-discharge relation is based upon measurements made by the slope-area method or upon a study of the conveyance characteristics of the stream channel. Where the stage-discharge relation is affected by backwater, the slope of the stream is commonly used as a second variable in computing discharge, and the peak discharge and peak stage do not necessarily occur at the same time. For these floods the peak discharge and the peak gage height have been tabulated as separate items.

Some of the figures of peak discharge shown in this report are not in agreement with figures published in the U. S. Geological Survey Water-Supply Papers. Most of these revisions are based upon data obtained after the discharge records were originally published. If the revised figures differ considerably from figures previously published, the appropriate changes will be noted as revisions in subsequent Water-Supply Papers.

Unless otherwise noted, the streamflow data in this report were collected by the U. S. Geological Survey in cooperation with many State and municipal agencies, the Corps of Engineers, and the Florida Power Corporation. When records collected by other agencies or private organizations are used, credit is given under the heading "Records available" in the manuscript.

In the station description the present site of the gaging station is described in "Location"; previous locations are described under "Gage". Where there has been a significant change in location, the appropriate notes have been shown in the tabulation of the annual flood peaks. 


\section{Sat11la River Basin}

(1) Sat1lla River near Waycross, Ga.

Location.--Iat $31^{\circ} 14^{\prime}$, long $82^{\circ} 19^{\prime}$, on downstream side of bridge pier near center of span on State Route 38,3 miles northeast of Waycross, Ware County, and 16 miles upstream from Alabama R1ver.

Drainage area. $--1,300 \mathrm{sq} \mathrm{m} 1$, approximately.

Records ava1lable.--U. S. Geological Survey: March 1937 to December 1953, da1ly gage he1ghts and discharges.

Gage.-Water-stage recorder. Datum of gage is $66.43 \mathrm{ft}$ above mean sea level, datum of 1929 , supplementary adjustment of 1936. Prior to Nov. 22, 1952, staff gage at s1te 300 ft downstream at same datum.

Stage-discharge relation.--Defined by current-meter measurements below 23,000 cfs and by slopeconveyance determination at $39,000 \mathrm{cfs}$.

H1storical data.--The flood of September 1928 reached a stage of 22.2 ft, from 1 nformation by pumping-station operator. This flood was reported by a local newspaper to be the highest known to the oldest settlers of the area.

Annual peak stages and discharges

\begin{tabular}{|c|c|c|c|c|c|c|c|}
\hline $\begin{array}{c}\text { Calendar } \\
\text { year }\end{array}$ & Date & $\begin{array}{c}\text { Gage } \\
\text { height } \\
\text { (feet) }\end{array}$ & $\begin{array}{c}\text { Discharge } \\
\text { (cfs) }\end{array}$ & $\begin{array}{c}\text { Calendar } \\
\text { year }\end{array}$ & Date & $\begin{array}{c}\text { Gage } \\
\text { height } \\
\text { (feet) }\end{array}$ & $\begin{array}{c}\text { Discharge } \\
\text { (cfs) }\end{array}$ \\
\hline $\begin{array}{l}1928 \\
1937 \\
1938 \\
1939 \\
1940 \\
1941 \\
1942 \\
1943 \\
1944 \\
1945\end{array}$ & $\begin{array}{lr}\text { Sept. } & - \\
& \\
\text { Apr. } & 11 \\
\text { July } & 30 \\
\text { Mar. } & 4 \\
\text { Feb. } & 23 \\
& \\
\text { Mar. } & 29,30 \\
\text { Jan. } & 6 \\
\text { Mar. } & 12 \\
\text { Mar. } & 10 \\
\text { Aug. } & 25\end{array}$ & $\begin{array}{l}22.2 \\
16.2 \\
14.1 \\
17.0 \\
14.2 \\
10.8 \\
17.6 \\
13.0 \\
18.0 \\
15.1\end{array}$ & $\begin{array}{r}37,000 \\
9,240 \\
4,160 \\
11,000 \\
4,300 \\
1,960 \\
12,800 \\
3,120 \\
13,400 \\
5,560\end{array}$ & $\begin{array}{l}1946 \\
1947 \\
1948 \\
1949 \\
1950 \\
1951 \\
1952 \\
1953\end{array}$ & $\begin{array}{lr}\text { July } & 31 \\
\text { Apr. } & 21 \\
\text { Apr. } & 4 \\
\text { Sept. } & 4 \\
\text { July } & 15 \\
& \\
\text { Apr. } & 4 \\
\text { Mar. } & 4,5,6 \\
\text { Oct. } & 1\end{array}$ & $\begin{array}{l}14.6 \\
16.5 \\
22.4 \\
18.1 \\
13.4 \\
16.2 \\
12.7 \\
18.1\end{array}$ & $\begin{array}{r}4,820 \\
8,520 \\
39,000 \\
13,800 \\
3,450 \\
7,770 \\
2,870 \\
13,800\end{array}$ \\
\hline
\end{tabular}

(2) Satilla River at Atkinson, Ga.

Location.--Lat $31^{\circ} 13^{\prime}$, long $81^{\circ} 52^{\prime}$, on downstream side of right pier of bridge on U. S. Highway 84,400 ft downstream from Atlantic Coast Line Ra1lroad bridge and 1 mile west of Atkinson, Brantley County.

Drainage area. $--2,880 \mathrm{sq} \mathrm{ml}$, approximately.

Records avallable.--U. S. Geological Survey: October 1931 to December 1953, da1ly gage he1ghts and discharges.

Gage.-Water-stage recorder. Datum of gage $1 \mathrm{~s} 14.79 \mathrm{ft}$ above mean sea level, datum of 1929 , supplementary adjustment of 1936. Prior to Dec. 5, 1933, staff gage at same s1te and datum.

Stage-discharge relation.--Defined by current-meter measurements below 68,000 cfs.

H1storical data.--Maximum stage known, $27.2 \mathrm{ft}$, from 1nformation by local res1dents, in September 1928.

Remarks.--Peak stage of Jan. 26, 1931, from records by Corps of Eng1neers. 
Sat1lia River Basin

(2) Satilla River at Atkinson, Ga.--Continued

Annual peak stages and discharges

\begin{tabular}{|c|c|c|c|c|c|c|c|}
\hline $\begin{array}{l}\text { Calendar } \\
\text { year }\end{array}$ & Date & $\begin{array}{l}\text { Gage } \\
\text { height } \\
\text { (feet) }\end{array}$ & $\begin{array}{l}\text { Discharge } \\
\text { (cfs) }\end{array}$ & $\begin{array}{l}\text { Calendar } \\
\text { year }\end{array}$ & Date & $\begin{array}{l}\text { Gage } \\
\text { height } \\
\text { (feet) }\end{array}$ & $\begin{array}{l}\text { Discharge } \\
\text { (cfs) }\end{array}$ \\
\hline 1928 & Sept. - & 27.2 & 110,000 & $\begin{array}{l}1942 \\
1943\end{array}$ & $\begin{array}{l}\text { Jan. } \\
\text { Mar. } \quad 17,18\end{array}$ & $\begin{array}{l}18.8 \\
13.0\end{array}$ & $\begin{array}{r}26,600 \\
5,650\end{array}$ \\
\hline $\begin{array}{l}1931 \\
1932 \\
1933\end{array}$ & $\begin{array}{ll}\text { Jan. } & 26 \\
\text { Aug. } & 31 \\
\text { Feb. } & 18\end{array}$ & $\begin{array}{l}13.0 \\
15.4 \\
16.96\end{array}$ & $\begin{array}{r}5,510 \\
12,200 \\
15,200\end{array}$ & $\begin{array}{l}1944 \\
1945\end{array}$ & $\begin{array}{ll}\text { Mar. } & 31 \\
\text { Aug. } & 27\end{array}$ & $\begin{array}{l}18.3 \\
15.3\end{array}$ & $\begin{array}{l}26,100 \\
11,200\end{array}$ \\
\hline $\begin{array}{l}1934 \\
1935\end{array}$ & $\begin{array}{l}\text { June } 11 \\
\text { Sept. } 18\end{array}$ & $\begin{array}{l}15.79 \\
16.25\end{array}$ & $\begin{array}{l}11,900 \\
13,000\end{array}$ & $\begin{array}{l}1946 \\
1947 \\
1948\end{array}$ & $\begin{array}{lr}\text { Jan. } & 7 \\
\text { Apr. } & 25 \\
\text { Apr. } & 6\end{array}$ & $\begin{array}{l}14.4 \\
16.0 \\
23.9\end{array}$ & $\begin{array}{r}8,500 \\
13,800 \\
68,100\end{array}$ \\
\hline $\begin{array}{l}1936 \\
1937 \\
1938\end{array}$ & $\begin{array}{ll}\text { Feb. } & 17 \\
\text { Apr. } & 14 \\
\text { Aug. } & 5,6\end{array}$ & $\begin{array}{l}14.5 \\
16.27 \\
13.68\end{array}$ & $\begin{array}{r}8,770 \\
13,300 \\
7,140\end{array}$ & $\begin{array}{l}1949 \\
1950\end{array}$ & $\begin{array}{l}\text { Sept. } 5 \\
\text { Sept. II }\end{array}$ & $\begin{array}{l}19.6 \\
16.6 .\end{array}$ & $\begin{array}{l}33,200 \\
15,500\end{array}$ \\
\hline $\begin{array}{l}1939 \\
1940\end{array}$ & $\begin{array}{l}\text { Mar: } \quad 7 \\
\text { Feb. } 26,27\end{array}$ & $\begin{array}{l}17.54 \\
14.5\end{array}$ & $\begin{array}{r}17,000 \\
7,920\end{array}$ & $\begin{array}{l}1951 \\
1952\end{array}$ & $\begin{array}{l}\text { Apr. } 9 \\
\text { Mar. II }\end{array}$ & $\begin{array}{l}16.0 \\
12.9\end{array}$ & $\begin{array}{r}13,000 \\
5,360\end{array}$ \\
\hline 1941 & July 27 & & 6,080 & 1953 & oct. & 19.8 & 34,600 \\
\hline
\end{tabular}

\section{St. Marys R1ver Basin}

(3) North Prong St. Marys River at Moniac, Ga.

Location.--Lat $30^{\circ} 31^{\prime}$, long $82^{\circ} 14^{\prime}$, in sec. 8, T. I N., R. 21 E., near right bank at upstream side of bridge on State Highway $94,950 \mathrm{ft}$ upstream from Georgia Southern and.Florida Ra1lway bridge, $0.5 \mathrm{mile}$ west of Moniac, and $1.0 \mathrm{mile}$ downstream from Moccasin Creek.

Drainage area.--About $160 \mathrm{sq} \mathrm{ml}$, includes part of watershed in Okefenokee Swamp wh1ch is Indefinite.

Records avallable.--U. S. Geological Survey: January 1921 to December 1923, January 1927 to June 1930, July 1932 to June 1934, October 1950 to December 1953, dally gage helghts and discharges.

Gage.--Water-stage recorder. Datum of gage is $89.40 \mathrm{ft}$ above mean sea level, datum of 1929. January 1921 to June 1934 , staff gage $800 \mathrm{ft}$ downstream at datum $3.22 \mathrm{ft}$ higher. 0ct. 3 to Dec. 13, 1950, w1re-weight gage at present site and datum.

Stage discharge relation.--Defined by current-meter measurements below $3,700 \mathrm{cfs}$.

Remarks.--Peak stage for flood of Sept. 19, 1928, determined from high-water mark; discharge estimated.

Annual peak stages and discharges

\begin{tabular}{|c|c|c|c|c|c|c|c|}
\hline $\begin{array}{c}\text { Calendar } \\
\text { year }\end{array}$ & Date & $\begin{array}{l}\text { Gage } \\
\text { height } \\
\text { (feet) }\end{array}$ & $\begin{array}{c}\text { Discharge } \\
\text { (cfs) }\end{array}$ & $\begin{array}{c}\text { Calendar } \\
\text { year }\end{array}$ & Date & $\begin{array}{l}\text { Gage } \\
\text { height } \\
\text { (feet) }\end{array}$ & $\begin{array}{l}\text { Discharge } \\
\text { (cfs) }\end{array}$ \\
\hline $\begin{array}{l}1921 \\
1922 \\
1923\end{array}$ & $\begin{array}{lc}\text { Aug. } & 2 \\
\text { Oct. } & 19 \\
\text { Jan. } & 7,8\end{array}$ & $\begin{array}{l}- \\
7.0 \\
7.0\end{array}$ & $\begin{array}{r}2,200 \\
670 \\
670\end{array}$ & $\begin{array}{l}1932 \\
1933 \\
1934 \\
\end{array}$ & $\begin{array}{lr}\text { Sept. } & 16 \\
\text { Feb. } & 9 \\
\text { June } & 18 \\
\end{array}$ & $\begin{array}{r}10.20 \\
9.89 \\
7.00 \\
\end{array}$ & $\begin{array}{r}1,870 \\
1,770 \\
550 \\
\end{array}$ \\
\hline $\begin{array}{l}1927 \\
1928 \\
1929 \\
\end{array}$ & $\begin{array}{lr}\text { July } & 23 \\
\text { Sept. } & 19 \\
\text { Oct. } & 2 \\
\end{array}$ & $\begin{array}{l}8.2 \\
16.7 \\
11.51 \\
\end{array}$ & $\begin{array}{l}1,100 \\
6,060 \\
2,640 \\
\end{array}$ & $\begin{array}{l}1951 \\
1952 \\
1953\end{array}$ & $\begin{array}{lr}\text { Dec. } & 28 \\
\text { Mar. } & 14 \\
\text { Oct. } & 1 \\
\end{array}$ & $\begin{array}{l}10.01 \\
12.36 \\
14.27 \\
\end{array}$ & $\begin{array}{r}492 \\
1,330 \\
2,140 \\
\end{array}$ \\
\hline
\end{tabular}




\section{St. Marys R1ver Basin}

(4) St. Marys R1ver near Macclenny, Fla.

Location.--Lat $30^{\circ} 21^{\prime} 35^{\prime \prime}$, long $82^{\circ} 04^{\prime} 55^{\prime \prime}$, In sec. 2, T. 2 S., R. 22 E., on right bank, $200 \mathrm{ft}$ downstream from slte of former Stokes Brldge on county road, 1 mile downstream from confluence of North and South Prongs, and 6 miles northeast of Macclenny.

Dra1nage area. $--720 \mathrm{sq} \mathrm{ml}$, approximately, 1ncludes part of watershed in okefenokee Swamp wh1ch is indeterminate.

Records avallable.--U. S. Geological Survey: October 1926 to December 1953, dally gage he1ghts and discharges.

Gage.-Water-stage recorder. Datum of gage is $40.00 \mathrm{ft}$ above mean sea level (levels by Mees and Mees). Prior to $\mathrm{Feb} .21,1939$, staff gage at site of former bridge $200 \mathrm{ft}$ upstream at same datum. Feb. 21, 1939, to Aug. 15, 1948, water-stage recorder on downstream wing wall of former bridge $200 \mathrm{ft}$ upstream at same dátum.

Stage-discharge relation.--Defined by current-meter measurements below 24,000 cfs.

Annual peak stages and discharges

\begin{tabular}{|c|c|c|c|c|c|c|c|}
\hline $\begin{array}{c}\text { Calendar } \\
\text { year }\end{array}$ & Date & $\begin{array}{l}\text { Gage } \\
\text { height } \\
\text { (feet) }\end{array}$ & $\begin{array}{l}\text { Discharge } \\
\quad \text { (cfs) }\end{array}$ & $\begin{array}{c}\text { Calendar } \\
\text { year }\end{array}$ & Date & $\begin{array}{c}\text { Gage } \\
\text { height } \\
\text { (feet) }\end{array}$ & $\begin{array}{l}\text { Discharge } \\
\text { (cfs) }\end{array}$ \\
\hline $\begin{array}{l}1927 \\
1928 \\
1929 \\
1930\end{array}$ & $\begin{array}{l}\text { Aug. } 24 \\
\text { Sept. } 20 \\
\text { Oct. } \\
\text { Mar. } 25\end{array}$ & $\begin{array}{l}12.4 \\
21.9 \\
18.18 \\
17.30\end{array}$ & $\begin{array}{r}3,070 \\
26,100 \\
11,400 \\
9,760\end{array}$ & $\begin{array}{l}1941 \\
1942 \\
1943 \\
1944 \\
1945\end{array}$ & $\begin{array}{lr}\text { Dec. } & 29 \\
\text { Jan. } & 6 \\
\text { Aug. } & 21 \\
\text { Oct. } & 21 \\
\text { Aug. } & 22\end{array}$ & $\begin{array}{r}13.50 \\
15.36 \\
8.98 \\
19.67 \\
16.00\end{array}$ & $\begin{array}{r}4,240 \\
6,800 \\
1,450 \\
16,100 \\
7,750\end{array}$ \\
\hline $\begin{array}{l}1931 \\
1932 \\
1933 \\
1934 \\
1935\end{array}$ & $\begin{array}{lr}\text { Apr. } & 7 \\
\text { Sept. } & 18 \\
\text { Apr. } & 17 \\
\text { June } & 18 \\
\text { Sept. } & 8\end{array}$ & $\begin{array}{l}10.62 \\
14.10 \\
14.38 \\
16.39 \\
14.20\end{array}$ & $\begin{array}{l}2,080 \\
4,860 \\
5,250 \\
8,260 \\
4,990\end{array}$ & $\begin{array}{l}1946 \\
1947 \\
1948 \\
1949 \\
1950\end{array}$ & $\begin{array}{lr} & \\
\text { Aug. } & 2 \\
\text { Sept. } & 25 \\
\text { Apr. } & 3 \\
\text { Feb. } & 8 \\
\text { Sept. } & 7\end{array}$ & $\begin{array}{l}15.13 \\
22.29 \\
21.97 \\
16.58 \\
21.96\end{array}$ & $\begin{array}{r}6,260 \\
28,100 \\
26,600 \\
8,230 \\
26,600\end{array}$ \\
\hline $\begin{array}{l}1936 \\
1937 \\
1938 \\
1939 \\
1940 \\
\end{array}$ & $\begin{array}{lr}\text { Feb. } & 23 \\
\text { Oct. } & 4 \\
\text { Aug. } & 8 \\
\text { July } & 12 \\
\text { Feb. } & 20\end{array}$ & $\begin{array}{r}9.40 \\
16.00 \\
16.25 \\
13.15 \\
11.11\end{array}$ & $\begin{array}{l}1,540 \\
7,580 \\
7,920 \\
3,770 \\
2,250\end{array}$ & $\begin{array}{l}1951 \\
1952 \\
1953\end{array}$ & $\begin{array}{lr}\text { Dec. } & 29 \\
\text { Feb. } & 28 \\
\text { Oct. } & 2\end{array}$ & $\begin{array}{r}6.74 \\
10.23 \\
18.27\end{array}$ & $\begin{array}{r}735 \\
1,810 \\
11,900\end{array}$ \\
\hline
\end{tabular}

St. Johns R1ver Basin

(5) St. Johns River headwaters near Vero Beach, Fla.

Location.--Iat $27^{\circ} 38^{\prime}$, long $80^{\circ} 40^{\prime}$, in sec. 6 , T. $33 \mathrm{~S} .$, R. $37 \mathrm{E}$., on upstream side of right abutment of bridge on State Highway $60,16 \frac{3}{4}$ miles west of c1ty hall in Vero Beach.

Drainage area.--203 sq $\mathrm{m} 1$.

Records ava1lable.--U. S. Geological Survey: February 1942 to December 1953, da1ly gage helghts.

Gage.--Water-stage recorder. Datum of gage $1 \mathrm{~s} 18.56 \mathrm{ft}$ above mean sea level, datum of 1929 (levels by Corps of Englneers).

Stage-d1scharge relation.--Not defined. 
St. Johns River Basin

(5) St. Johns River headwaters near Vero Beach, Fla.--Continued

Annual peak stages

\begin{tabular}{|c|c|c|c|c|c|}
\hline Calendar year & Date & $\begin{array}{c}\text { Gage he 1ght } \\
\text { (feet) }\end{array}$ & Calendar year & Date & $\begin{array}{c}\text { Gage helght } \\
(\text { feet) }\end{array}$ \\
\hline $\begin{array}{l}1942 \\
1943 \\
1944 \\
1945\end{array}$ & $\begin{array}{ll}\text { Mar. } & 19 \\
\text { Oct. } & 15 \\
\text { Nov. } & 2,4 \\
\text { Sept. } & 22\end{array}$ & $\begin{array}{l}6.76 \\
6.83 \\
6.94 \\
7.31\end{array}$ & $\begin{array}{l}1948 \\
1949 \\
1950 \\
1951\end{array}$ & $\begin{array}{l}\text { Oct. }-\overline{1} \\
\text { Oct. } 11 \\
\text { Oct. } 23 \\
\text { Oct. } 24\end{array}$ & $\begin{array}{l}8.44 \\
8.03 \\
7.71 \\
7.02\end{array}$ \\
\hline $\begin{array}{l}1946 \\
1947 \\
\end{array}$ & $\begin{array}{lr}\text { Oct. } & 8 \\
\text { oct. } & 12 \\
\end{array}$ & $\begin{array}{l}6.43 \\
8.81 \\
\end{array}$ & $\begin{array}{l}1952 \\
1953 \\
\end{array}$ & $\begin{array}{l}\text { Oct. } 27,28 \\
\text { Oct. } 14\end{array}$ & $\begin{array}{l}7.62 \\
8.63 \\
\end{array}$ \\
\hline
\end{tabular}

(6) St. Johns River headwaters near Kenansville, Fla.

Location.--Lat $27^{\circ} 49^{\prime}$, long $80^{\circ} 49^{\prime}$, in sec. 2, T. 31 S., R. 35 E., near left bank on upstream slde of county road between Kenansville and Fellsmere, il $\frac{1}{2}$ miles east of Kenansville.

Dralnage area. $--442 \mathrm{sq} \mathrm{ml}$.

Records avallable.--U. S. Geologlcal Survey: February 1942 to December 1953, dally gage helghts. Gage.--Water-stage recorder. Datum of gage is $17.02 \mathrm{ft}$ above mean sea level, datum of 1929. Stage-discharge relation.--Not defined.

Annual peak stages

\begin{tabular}{|c|c|c|c|c|c|}
\hline Calendar year & Date & $\begin{array}{l}\text { Gage helght } \\
\text { feet) }\end{array}$ & Calendar year & Date & $\begin{array}{c}\text { Gage he1ght } \\
\text { feet) }\end{array}$ \\
\hline $\begin{array}{l}1942 \\
1943 \\
1944 \\
1945 \\
1946 \\
1947 \\
\end{array}$ & $\begin{array}{l}\text { June } 13 \\
\text { Oct. } 8,9 \\
\text { Oct. 19, } \\
\text { Sept. } 20,21 \\
\text { Sept. - } \\
\text { Sept. - }\end{array}$ & $\begin{array}{l}6.99 \\
7.85 \\
7.40 \\
6.93 \\
8.38 \\
\end{array}$ & $\begin{array}{l}1948 \\
1949 \\
1950 \\
1951 \\
1952 \\
1953 \\
\end{array}$ & $\begin{array}{l}\text { Oct. }-\overline{3} \\
\text { Oct. } 3-6 \\
\text { Oct. } 19 \\
\text { Nov. } 23-27 \\
\text { Oct. } 27,28 \\
\text { Oct. } 12\end{array}$ & $\begin{array}{l}8.52 \\
7.65 \\
7.46 \\
6.52 \\
7.40 \\
8.33 \\
\end{array}$ \\
\hline
\end{tabular}

(7) St. Johns Rlver near Melbourne, Fla.

Location.--Lat $28^{\circ} 05^{\prime} 03^{\prime \prime}$, long $80^{\circ} 45^{\prime} 11^{\prime \prime}$, In NE $\frac{1}{4} \mathrm{sec} .6, \mathrm{~T} .28 \mathrm{~S} ., \mathrm{R} .36 \mathrm{E}$, on left bank $10 \mathrm{ft}$ upstream from bridge on U. S. Highway $192,1.0$ mile downstream from Sawgrass Lake, 1.8 m1les upstream from Lake Wash1ngton, and 9.2 miles west of Melbourne.

Drainage area. $--874 \mathrm{sq} \mathrm{ml}$.

Records avallable.--U. S. Geologlcal Survey: November 1939 to December 1953, da1ly gage helghts and discharges:

Gage.--Water-stage recorder. Datum of gage is $11.22 \mathrm{ft}$ above mean sea level, datum of 1929. Prior to July 26, 1940, staff gage at same site and datum.

Stage-discharge relation.--Defined by current-meter measurements below 8,800 cfs. H1gh-water shifts have occurred. 
St. Johns River Basin

(7) St. Johns River near Melbourne, Fla.--Continued

Annual peak stages and discharges

\begin{tabular}{c|c|c|c|c|c|c|c}
\hline $\begin{array}{c}\text { Calendar } \\
\text { year }\end{array}$ & Date & $\begin{array}{c}\text { Gage } \\
\text { height } \\
\text { (feet) }\end{array}$ & $\begin{array}{c}\text { Discharge } \\
\text { (cfs) }\end{array}$ & $\begin{array}{c}\text { Calendar } \\
\text { year }\end{array}$ & Date & $\begin{array}{c}\text { Gage } \\
\text { height } \\
\text { (feet) }\end{array}$ & $\begin{array}{c}\text { Discharge } \\
\text { (cfs) }\end{array}$ \\
\hline 1940 & Sept. 19 & 5.96 & 675 & 1947 & Oct. 1 & 9.16 & 7,350 \\
1941 & Nov. 19-21 & 7.73 & 3,730 & 1948 & Oct. 2 & 9.33 & 8,160 \\
1942 & July 4,5 & 6.94 & 1,360 & 1949 & Oct. 5. 6 & 8.56 & 5,600 \\
1943 & Oct. 13 & 7.36 & 2,320 & Oct. 23 & 8.45 & 5,350 \\
1944 & Oct. 26, 27 & 7.75 & 3,800 & 1951 & Oct. 9 & 7.23 & 2,520 \\
1945 & Sept. 22 & 8.04 & 4,360 & 1952 & Oct. 28 & 8.39 & 4,780 \\
1946 & Aug. 9 & 7.21 & 3,060 & 1953 & Oct. 12 & 9.47 & 8,850 \\
\hline
\end{tabular}

(8) Lake Washington near Eau Gallie, Fla.

Location.--Lat $28^{\circ} 09^{\prime}$, long $80^{\circ} 44^{\prime}$, near sec. I1ne 8 and 9, T. $27 \mathrm{~S} ., \mathrm{R}, 36 \mathrm{E}$., in canal on east shore of lake, at f1sh camp known as Lake Wash1ngton Resort, Inc., $6 \frac{1}{2}$ miles west of Eau Gall1e.

Records avallable.--U. S. Geological Survey: July 1942 to December 1953, da1ly gage helghts.

Gage.--Water-stage recorder. Datum of gage $1812.39 \mathrm{ft}$ above mean sea level, datum of 1929 . Prior to July 21, 1950, gage was attached to dock in lake at same s1te and datum.

Annual peak stages

\begin{tabular}{|c|c|c|c|c|c|}
\hline Calendar year & Date & $\begin{array}{c}\text { Gage he1ght } \\
\text { (feet) }\end{array}$ & Calendar year & Date & $\begin{array}{c}\text { Gage he1ght } \\
\text { feet) }\end{array}$ \\
\hline $\begin{array}{l}1942 \\
1943 \\
1944 \\
1945\end{array}$ & $\begin{array}{lr}\text { July } & 26 \\
\text { Oct. } & 8 \\
\text { Oct. } & 29,30 \\
\text { Sept. } & 28\end{array}$ & $\begin{array}{l}5.24 \\
5.72 \\
5.88 \\
5.94\end{array}$ & $\begin{array}{l}1948 \\
1949 \\
1950\end{array}$ & $\begin{array}{l}\text { Oct. 2, } 3 \\
\text { Oct. 11 } \\
\text { Oct. - }\end{array}$ & $\begin{array}{l}7.43 \\
6.73 \\
6.32\end{array}$ \\
\hline $\begin{array}{l}1946 \\
1947 \\
\end{array}$ & $\begin{array}{lr}\text { Aug. } & 11 \\
\text { Oct. } & 5 \\
\end{array}$ & $\begin{array}{l}5.36 \\
7.32 \\
\end{array}$ & $\begin{array}{l}1952 \\
1952 \\
1953 \\
\end{array}$ & $\begin{array}{l}\text { Oct. } 28,29 \\
\text { Oct. } 12,13 \\
\end{array}$ & $\begin{array}{l}5.65 \\
6.48 \\
7.68 \\
\end{array}$ \\
\hline
\end{tabular}

(9) Lake Poinsett near Cocoa, Fla.

Location.--Lat $28^{\circ} 21^{\prime}$, long $80^{\circ} 49^{\prime}$, in SW $\frac{1}{4} \mathrm{sec} .34, \mathrm{~T} .24 \mathrm{~S} ., \mathrm{R} .35 \mathrm{E}$, on p1le at northeast end of boat shed on canal at flshing camp on northeast shore of lake, $5 \frac{1}{2} \mathrm{miles}$ west of cocoa.

Records avallable.--U. S. Geolog1cal Survey: November 1941 to December 1953, da1ly gage helghts.

Gage.--Staff gage read once da1ly. Datum of gage 1s $5.06 \mathrm{ft}$ above mean sea level, datum of 1929.

Prior to Feb. 23, 1943, at datum $4.32 \mathrm{ft}$ higher. 
St. Johns River Basin

(9) Lake Poinsett near Cocoa, Fla.--Continued

Annual peak stages

\begin{tabular}{|c|c|c|c|c|c|}
\hline Calendar year & Date & $\begin{array}{l}\text { Gage height } \\
\text { feet) }\end{array}$ & Calendar year & Date & $\begin{array}{l}\text { Gage he1ght } \\
\text { (feet) }\end{array}$ \\
\hline $\begin{array}{l}1942 \\
1943 \\
1944 \\
1945 \\
1946 \\
1947\end{array}$ & $\begin{array}{lr}\text { Jan. } & 9 \\
\text { Oct. } & 12 \\
\text { Oct. } & 22 \\
\text { Sept. } & 19 \\
\text { Sept. } & 20-22 \\
\text { Oct. } & 7 \\
\end{array}$ & $\begin{array}{r}09.26 \\
10.54 \\
10.10 \\
10.48 \\
9.12 \\
11.41 \\
\end{array}$ & $\begin{array}{l}1948 \\
1949 \\
1950 \\
1951 \\
1952 \\
1953 \\
\end{array}$ & $\begin{array}{ll}\text { Oct. } & 5-8 \\
\text { Oct. } & 1 \\
\text { Oct. } & 28-31 \\
\text { Oct. } 5,6 \\
\text { Oct. } 27 \text { to Nov. } 1 \\
\text { Oct. } 11\end{array}$ & $\begin{array}{l}12.48 \\
11.46 \\
10.04 \\
10.30 \\
10.60 \\
12.50\end{array}$ \\
\hline
\end{tabular}

(10) St. Johns River near Christmas, Fla.

Location.--Lat $28^{\circ} 32^{\prime} 35^{\prime \prime}$, long $80^{\circ} 56^{\prime} 40^{\prime \prime}$, in SW $\frac{1}{4}$ sec. 29, T. 22 S., R. 34 E., on left bank about $15 \mathrm{ft}$ downstream from bridge on State Highway 50, 2 miles upstream from Lake Cone and Tosohatchee Creek, and 4.5 miles east of Christmas.

Drainage area. $--1,418 \mathrm{sq} \mathrm{ml}$.

Records avallable.--U. S. Geological Survey: December 1933 to December 1953, da1ly gage heights and discharges.

Gage.--Water-stage recorder. Datum of gage is $1.62 \mathrm{ft}$ above mean sea level, datum of 1929 . Prior to July 23, 1934, staff gage at same site and datum.

Stage discharge relation.--Defined by current-meter measurements below 11,500 cfs. Minor highwater shifts have occurred.

Annual peak stages and discharges

\begin{tabular}{|c|c|c|c|c|c|c|c|}
\hline $\begin{array}{c}\text { Calendar } \\
\text { year }\end{array}$ & Date & $\begin{array}{l}\text { Gage } \\
\text { height } \\
\text { (feet) }\end{array}$ & $\begin{array}{c}\text { Discharge } \\
\text { (cfs) }\end{array}$ & $\begin{array}{l}\text { Calendar } \\
\text { year }\end{array}$ & Date & $\begin{array}{l}\text { Gage } \\
\text { height } \\
\text { (feet) }\end{array}$ & $\begin{array}{l}\text { Discharge } \\
\quad \text { (cfs) }\end{array}$ \\
\hline $\begin{array}{l}1934 \\
1935\end{array}$ & $\begin{array}{ll}\text { June } & 20 \\
\text { Oct. } & 16\end{array}$ & $\begin{array}{l}8.9 \\
8.56\end{array}$ & $\begin{array}{l}4,800 \\
4,700\end{array}$ & $\begin{array}{l}1944 \\
1945\end{array}$ & $\begin{array}{ll}\text { Oct. } & 22 \\
\text { Sept. } & 21\end{array}$ & $\begin{array}{l}8.32 \\
9.14\end{array}$ & $\begin{array}{l}4,270 \\
9,230\end{array}$ \\
\hline $\begin{array}{l}1936 \\
1937 \\
1938 \\
1939 \\
1940\end{array}$ & $\begin{array}{ll}\text { Mar. } & 12,13 \\
\text { Dec. } & 1,2 \\
\text { July } & 17 \\
\text { Sept. } & 29,30 \\
\text { Oct. } & 1\end{array}$ & $\begin{array}{l}7.83 \\
8.17 \\
6.60 \\
7.66 \\
7.00\end{array}$ & $\begin{array}{l}4,120 \\
3,620 \\
1,260 \\
2,860 \\
2,120\end{array}$ & $\begin{array}{l}1946 \\
1947 \\
1948 \\
1949 \\
1950\end{array}$ & $\begin{array}{l}\text { Aug. } 31 \\
\text { Oct. } 12,13 \\
\text { Oct. } 9 \\
\text { Oct. } 5-9 \\
\text { Oct. } 20,23\end{array}$ & $\begin{array}{l}7.01 \\
9.68 \\
9.86 \\
- \\
8.22\end{array}$ & $\begin{array}{r}3,500 \\
10,700 \\
10,200 \\
a 6,600 \\
4,910\end{array}$ \\
\hline $\begin{array}{l}1941 \\
1942 \\
1943 \\
\end{array}$ & $\begin{array}{l}\text { July } 28,29 \\
\text { July } 11 \\
\text { Sept. } 21-24\end{array}$ & $\begin{array}{l}8.82 \\
7.40 \\
-\end{array}$ & $\begin{array}{r}5,270 \\
2,350 \\
a 3,700\end{array}$ & $\begin{array}{l}1951 \\
1952 \\
1953\end{array}$ & $\begin{array}{lr}\text { Oct. } & 6 \\
\text { Oct. } & 29 \\
\text { Oct. } & 12\end{array}$ & $\begin{array}{c}8.0 \\
8.36 \\
10.59\end{array}$ & $\begin{array}{r}4,860 \\
5,750 \\
11,700\end{array}$ \\
\hline
\end{tabular}




\section{St. Johns River Basin}

(11) Econlockhatchee River near Chuluota, Fla.

Location.--Lat $28^{\circ} 40^{\prime} 40^{\prime \prime}$, long $81^{\circ} 06^{\prime} 50^{\prime \prime}$, in sec. 10, T. $21 \mathrm{~S}$., R. 32 E., on right bank, $10 \mathrm{ft}$ downstream from highway bridge, $2.6 \mathrm{miles}$ northeast of chuluota, and $10 \mathrm{miles}$ upstream from mouth.

Drainage area. $--260 \mathrm{sq} \mathrm{m}$, approximately.

Records ava1lable.--U. S. Geolog1cal Survey: November 1935 to December 1953, da1ly gage he1ghts. and discharges.

Gage.--Water-stage recorder. Datum of gage is $2.14 \mathrm{ft}$ above mean sea level (Corps of Englneers benchmark). Prior to May 18, 1939, staff gage at same site and datum.

Water-stage recorder for station on St. Johns R1ver above Lake Harney, near Geneva is used as an auxiliary gage for this station.

Stage-discharge relation.--Def1ned by current-meter measurements below 4,300 cfs; affected at times by backwater from St. Johns River. Since September 1943, discharge computed by using fall, as determined from auxillary gage, as a factor.

Remarks.--Records include some flow diverted from Lake Mary Jane in Lake Okeechobee and the Everglades basin through Econlockhatchee Headwaters Canal.

Annual peak stages and discharges

\begin{tabular}{|c|c|c|c|c|c|c|c|}
\hline $\begin{array}{l}\text { Calendar } \\
\text { year }\end{array}$ & Date & $\begin{array}{l}\text { Gage } \\
\text { height } \\
\text { (feet) }\end{array}$ & $\begin{array}{l}\text { Discharge } \\
\text { (cfs) }\end{array}$ & $\begin{array}{l}\text { Calendar } \\
\text { year }\end{array}$ & Date & $\begin{array}{c}\text { Gage } \\
\text { height } \\
\text { (feet) }\end{array}$ & $\begin{array}{l}\text { Discharge } \\
\text { (cfs) }\end{array}$ \\
\hline $\begin{array}{l}1936 \\
1937 \\
1938 \\
1939 \\
1940\end{array}$ & $\begin{array}{lr}\text { Oct. } & 15 \\
\text { Dec. } & 1 \\
\text { July } & 17 \\
\text { June } & 18 \\
\text { Aug. } & 6,7\end{array}$ & $\begin{array}{r}10.55 \\
10.57 \\
9.81 \\
11.61 \\
-\end{array}$ & $\begin{array}{r}1,760 \\
1,760 \\
1,490 \\
2,430 \\
950\end{array}$ & $\begin{array}{l}1946 \\
1947 \\
1948 \\
1949 \\
1950\end{array}$ & $\begin{array}{lr}\text { Aug. } & 5 \\
\text { Sept. } & 25 \\
\text { Sept. } & 24 \\
\text { Oct. } \quad 1 \\
\text { Oct. } \quad 20\end{array}$ & $\begin{array}{r}9.72 \\
13.87 \\
18.09 \\
13.28 \\
16.54\end{array}$ & $\begin{array}{r}1,200 \\
4,560 \\
10,000 \\
3,700 \\
7,350\end{array}$ \\
\hline $\begin{array}{l}1941 \\
1942 \\
1943 \\
1944 \\
1945\end{array}$ & $\begin{array}{lr}\text { July } & 14 \\
\text { July } & 9 \\
\text { Sept. } & 22 \\
\text { Oct. } & 21 \\
\text { Sept. } & 18\end{array}$ & $\begin{array}{r}- \\
9.43 \\
11.75 \\
14.96 \\
17.93\end{array}$ & $\begin{array}{l}2,160 \\
1,360 \\
2,000 \\
6,100 \\
9,040\end{array}$ & $\begin{array}{l}1951 \\
1952 \\
1953\end{array}$ & $\begin{array}{lr}\text { Oct. } & 5 \\
\text { Oct. } & 23 \\
\text { Sept. } & 6\end{array}$ & $\begin{array}{l}11.03 \\
12.00 \\
13.72\end{array}$ & $\begin{array}{l}2,000 \\
2,630 \\
4,080\end{array}$ \\
\hline
\end{tabular}

(12) St. Johns River above Lake Harney, near Geneva, Fla.

Location.--Lat $28^{\circ} 43^{\prime}$, Jong $81^{\circ} 02^{\prime}$, in sec. 33, T. 20 S., R. 33 E., near right bank at upstream s1de of bridge on State Highway 46, I mile upstream from Lake Harney, $5 \frac{1}{2}$ miles southeast of Geneva, and $15 \frac{1}{2}$ m1les southeast of Sanford.

Dra1nage area.--1,910 sq $\mathrm{m} 1$, approximately.

Records avallable.--Corps of Englneers: July 1941 to August 1943, dally gage helghts. U. S. Geological Survey: September 1943 to December 1953, da1ly gage helghts; July 1951 to December 1953, periodic discharge measurements.

Gage.--Water-stage recorder. Datum of gage is at mean sea level, datum of 1929 (Corps of Engineers benchmark). Prior to Sept. 4, 1943, staff gage at same site and datum.

Stage-d1scharge relation.--Not defined; affected by backwater from Lake Harney. 
St. Johns River Basin

(12) St. Johns River above Lake Harney, near Geneva, Fla.--Continued

Annual peak stages

\begin{tabular}{|c|c|c|c|c|c|}
\hline Calendar year & Date & $\begin{array}{l}\text { Gage height } \\
\text { feet) }\end{array}$ & Calendar year & Date & $\begin{array}{l}\text { Gage helght } \\
\text { feet) }\end{array}$ \\
\hline $\begin{array}{l}1941 \\
1942 \\
1943 \\
1944 \\
1945 \\
1946 \\
1947\end{array}$ & $\begin{array}{ll}\text { Aug. } & 1 \\
\text { Mar. } & 22-25 \\
\text { Sept. } & 25 \\
\text { Oct. } & 25 \\
\text { Sept. } & 21 \\
& \\
\text { Sept. } & 23 \\
\text { Oct. } & - \\
\end{array}$ & $\begin{array}{l}7.8 \\
4.8 \\
7.45 \\
7.81 \\
9.87 \\
6.53 \\
9.49 \\
\end{array}$ & $\begin{array}{l}1948 \\
1949 \\
1950 \\
1951 \\
1952 \\
1953\end{array}$ & $\begin{array}{l}\text { Oct. } 10 \\
\text { Oct. } 4 \\
\text { Oct. } 24 \\
\text { Oct. } 8 \\
\text { Oct. } 28 \\
\text { Oct. } 14\end{array}$ & $\begin{array}{r}9.72 \\
8.44 \\
8.29 \\
6.88 \\
7.52 \\
10.62\end{array}$ \\
\hline
\end{tabular}

(13) St. Johns River at Osceola, Fla.

Location.--Lat $28^{\circ} 48^{\prime}$, long $81^{\circ} 04^{\prime}$, in sec. 31 , T. $19 \mathrm{~S} .$, R. $33 \mathrm{E}$., on right bank on upstream fender piling of Fiorlda East Coast Railway bridge at Osceola, half a mile downstream from Lake Harney.

Drainage area. $--1,950 \mathrm{sq} \mathrm{m1}$, approximately.

Records avallable.--Corps of Engineers: August 1941 to August 1943, da1ly gage helghts. U. S. Geological Survey: September 1943 to July 1948, da1ly gage heights.

Gage.--Water-stage recorder. Datum of gage is at mean sea level, datum of 1929 (Corps of Engineers benchmark). Aug. 8, 1941, to Sept. 2, 1943, staff gage at same site and datum. Sept. 2, 1943, to Nov. 4, 1943, water-stage recorder at site a quarter of a mile downstream at same datum.

Stage-discharge relation.--Not defined.

Annual peak stages

\begin{tabular}{|c|c|c|c|c|c|}
\hline Calendar year & Date & $\begin{array}{c}\text { Gage height } \\
\text { (feet) }\end{array}$ & Calendar year & Date & $\begin{array}{l}\text { Gage helght } \\
\text { (feet) }\end{array}$ \\
\hline $\begin{array}{l}1941 \\
1942 \\
1943 \\
1944 \\
\end{array}$ & $\begin{array}{ll}\text { Aug. } & - \\
\text { Mar. } 24,25 \\
\text { Sept. } 25 \\
\text { Oct. } 26\end{array}$ & $\begin{array}{l}\text { (a) } \\
4.35 \\
(\mathrm{a}) \\
7.61\end{array}$ & $\begin{array}{l}1945 \\
1946 \\
1947\end{array}$ & $\begin{array}{l}\text { Sept. } 21 \\
\text { Sept. } 1 \overline{6} \\
\text { Oct. }\end{array}$ & $\begin{array}{l}9.62 \\
6.41 \\
9.22\end{array}$ \\
\hline
\end{tabular}

$\exists$ No gage-he1ght record. 


\section{St. Johns River Basin}

(14) Jessup Lake Outlet near Sanford, Fla.

Location.--Lat $28^{\circ} 47^{\prime}$, long $81^{\circ} 11^{\prime}$, in sec. I, T. $20 \mathrm{~S} ., \mathrm{R} .31 \mathrm{E}$. , near left bank on downstream side of bridge on State Highway $46,5 \frac{1}{4}$ miles southeast of Sanford.

Records avallable.--Corps of Engineers: July 1941 to August 1943, da1ly gage heights.

U. S. Geological Survey: August 1943 to July 1948, dally gage helghts.

Gage.--Water-stage recorder. Datum of gage 1s at mean sea level, datum of 1929 (Corps of Engineers benchmark). Prior to Aug. 28, 1943, staff gage at same site and datum.

Stage-discharge relation.--Not defined.

H1storical data.--Flood of July 1930 reached a stage of $8.4 \mathrm{ft}$, from information by local resident.

Annual peak stages

\begin{tabular}{|c|c|c|c|c|c|}
\hline Calendar year & Date & $\begin{array}{l}\text { Gage he 1ght } \\
\text { feet) }\end{array}$ & Calendar year & Date & $\begin{array}{l}\text { Gage helght } \\
\text { feet) }\end{array}$ \\
\hline $\begin{array}{l}1930 \\
1941 \\
1942 \\
1943\end{array}$ & $\begin{array}{l}\text { July }- \\
\text { Aug. } \quad 1-9 \\
\text { Mar. } \quad 21 \\
\text { Sept. - }\end{array}$ & $\begin{array}{r}8.4 \\
\\
5.8 \\
3.6 \\
5.8\end{array}$ & $\begin{array}{l}1944 \\
1945 \\
1946 \\
1947\end{array}$ & $\begin{array}{ll}\text { Oct. } & 28 \\
\text { Sept. } & 26 \\
& \\
\text { Sept. } & 26 \\
\text { Oct. } & 21\end{array}$ & $\begin{array}{l}6.10 \\
7.76 \\
5.03 \\
7.60\end{array}$ \\
\hline
\end{tabular}

(15) St. Johns River above Lake Monroe, near Sanford, Fla.

Location.--Lat $28^{\circ} 48^{\prime}$, long $81^{\circ} 13^{\prime}$, in sec. $27, T .19 \mathrm{~S} .$, R. 31 E., near left bank on downstream slde of bridge on State H1ghway 415 , about 2 miles upstream from Lake Monroe and $3 \frac{1}{4} \mathrm{mlles}$ east of Sanford.

Dra1nage area. $--2,320 \mathrm{sq} \mathrm{m} 1$, approximately.

Records ava1lable.--Corps of Englneers: July 1941 to August 1943, da1ly gage he1ghts. U. S. Geological Survey: August 1943 to July 1948, da1ly gage helghts.

Gage.--Water-stage recorder. Datum of gage 1s at mean sea level, datum of 1929 (Corps of Englneers benchmark). Prior to Aug. 28, 1943, staff gage at same site and datum.

Stage-d1scharge relation.--Not defined.

H1storical data.--Flood of October 1924 reached a stage of $8.5 \mathrm{ft}$ from 1nformation by local resident. See additional high-water data for Lake Monroe listed with data for following station (St. Johns Rlver near Sanford, Fla.).

Annual peak stages

\begin{tabular}{|c|c|c|c|c|c|}
\hline Calendar year & Date & $\begin{array}{c}\text { Gage he1ght } \\
\text { feet) }\end{array}$ & Calendar year & Date & $\begin{array}{l}\text { Gage he1ght } \\
\text { (feet) }\end{array}$ \\
\hline 1924 & oct. - & 8.5 & $\begin{array}{l}1944 \\
1945\end{array}$ & $\begin{array}{l}\text { Oct. } 29 \\
\text { Sept. } 28\end{array}$ & $\begin{array}{l}5.95 \\
7.62\end{array}$ \\
\hline $\begin{array}{l}1941 \\
1942 \\
1943 \\
\end{array}$ & $\begin{array}{ll}\text { Aug. } & 4-7 \\
\text { Jan. } & 3,4 \\
\text { Oct. } & 10\end{array}$ & $\begin{array}{l}5.1 \\
3.6 \\
5.92 \\
\end{array}$ & $\begin{array}{l}1946 \\
1947 \\
\end{array}$ & $\begin{array}{l}\text { Sept. } 26 \\
\text { Oct. } 22 \\
\end{array}$ & $\begin{array}{r}4.89 \\
\quad 7.42 \\
\end{array}$ \\
\hline
\end{tabular}




\section{St. Johns River Basin}

(16) St. Johns River near Sanford, Fla.

Location.--Lat $28^{\circ} 50^{\prime}$, long $81^{\circ} 19^{\prime}$, in sec. 16, T. $19 \mathrm{~S} ., \mathrm{R} .30 \mathrm{E}$, , on left bank 25 ft downstream from bridge on U. S. Highways 17 and 92 , near downstream end of Lake Monroe, and $4 \mathrm{~m} 1 \mathrm{les}$ northwest of Sanford.

Drainage area. $--2,420 \mathrm{sq} \mathrm{ml}$, approximately.

Records avallable.--Clty of Sanford: July 1920 to January 1940, once-weekly gage helghts. U. S. Geological Survey: August 1941 to December 1953, da1ly gage helghts and perlodic discharge measurements.

Gage.-Water-stage recorder. Datum of gage is $0.09 \mathrm{ft}$ below mean sea level, datum of 1929. Gage used by c1ty of Sanford was staff gage in Lake Monroe upstream from present site. Datum of gage was at mean sea level (levels by city engineer). (All gage helghts shown below have been reduced to elevations above mean sea level).

Stage-discharge relation.--Not defined; affected by backwater. Insufficient data for computation of annual discharge maxima.

Historical data.--Elevations for floods of 1871, 1880, 1898, and 1910, furnished by Mr. Fred T. Williams, former c1ty englneer for Sanford. (Several datum adjustments have been used in Southern Florida; 1 is assumed that these elevations have been adjusted to datum of 1929).

Annual peak stages

\begin{tabular}{|c|c|c|c|c|c|}
\hline Calendar year & Date & $\begin{array}{l}\text { Gage helght } \\
\text { (feet) }\end{array}$ & Calendar year & Date & $\begin{array}{c}\text { Gage height } \\
\text { (feet) }\end{array}$ \\
\hline 1871 & - & 11.78 & $\begin{array}{l}1934 \\
1935\end{array}$ & $\begin{array}{l}\text { June -, July - } \\
\text { Oct. }\end{array}$ & 6.0 \\
\hline 1880 & - & 13.28 & & & \\
\hline 1898 & - & 9.37 & $\begin{array}{l}1936 \\
1937\end{array}$ & $\begin{array}{ll}\text { Mar. } & - \\
\text { Dec. } & -\end{array}$ & $\begin{array}{l}4.0 \\
4.5\end{array}$ \\
\hline 1910 & - & 7.16 & $\begin{array}{l}1730 \\
1939\end{array}$ & oct. - & 3.5 \\
\hline 1920 & oct. - & 4.1 & 1941 & & \\
\hline $\begin{array}{l}1921 \\
1922 \\
1923 \\
1924 \\
1925\end{array}$ & $\begin{array}{l}\text { Nov. - } \\
\text { Nov. }- \\
\text { June -, July - } \\
\text { Oct. - } \\
\text { Sept. - }\end{array}$ & $\begin{array}{l}4.0 \\
5.2 \\
6.0 \\
8.06 \\
4.2\end{array}$ & $\begin{array}{l}1941 \\
1942 \\
1943 \\
1944 \\
1945\end{array}$ & $\begin{array}{ll}\text { Mar. } & 20,21 \\
\text { Oct. } 11 & \\
\text { Oct. } & 30 \\
\text { Sept. } 28,29\end{array}$ & $\begin{array}{l}5.38 \\
3.25 \\
5.69 \\
5.70 \\
7.30\end{array}$ \\
\hline $\begin{array}{l}1926 \\
1927 \\
1928 \\
1929 \\
1930\end{array}$ & $\begin{array}{l}\text { Aug. - } \\
\text { Oct. - } \\
\text { Oct. - } \\
\text { Oct. - } \\
\text { July - }\end{array}$ & $\begin{array}{l}7.10 \\
1.6 \\
6.5 \\
6.2 \\
8.16\end{array}$ & $\begin{array}{l}1946 \\
1947 \\
1948 \\
1949 \\
1950\end{array}$ & $\begin{array}{l}\text { Sept. } 27 \\
\text { Oct. } 22,23 \\
\text { Oct. } 13 \\
\text { Oct. } 11 \\
\text { Oct. } 29,30\end{array}$ & $\begin{array}{l}4.63 \\
7.14 \\
7.32 \\
5.91 \\
5.66\end{array}$ \\
\hline $\begin{array}{l}1931 \\
1932 \\
1933\end{array}$ & $\begin{array}{l}\text { Jan. - } \\
\text { June } \\
\text { Sept. - }\end{array}$ & $\begin{array}{l}3.4 \\
2.4 \\
5.4\end{array}$ & $\begin{array}{l}1951 \\
1952 \\
1953\end{array}$ & $\begin{array}{l}\text { Oct. } 18,25 \\
\text { Nov. } 3,4 \\
\text { Oct. } 15\end{array}$ & $\begin{array}{l}4.27 \\
4.97 \\
8.52\end{array}$ \\
\hline
\end{tabular}

a No gage-helght record. 


\section{St. Johns R1ver Basin}

(17) Wek1va River near Sanford, Fla.

Location.--Lat $28^{\circ} 49^{\prime}$, long $81^{\circ} 25^{\prime}$, on line between secs. 21 and $28, T .19 \mathrm{~S} ., \mathrm{R}, 29 \mathrm{E}$, , near right bank at downstream side of bridge on State Highway $46,4 \frac{1}{2}$ miles downstream from Little Wek1va RIver, $5 \frac{1}{2}$ miles upstream from mouth, and 9 miles west of Sanford.

Records ava1lable.--U. S. Geolog1cal Survey: October 1931 to September 1935, perlodic discharge measurements; October 1935 to December 1953, dally gage helghts and discharges.

Gage.--Staff gage read once da1ly. Datum of gage 1s not determined. Prior to Nov. 6, 1935, reference point at same site.

Stage-discharge relation.--Defined by current-meter measurements below 820 cfs. Considerable shifting at all stages and backwater effect from hyac1nths jams and the St. Johns R1ver.

Remarks.--Wek1va River is fed by numerous springs.

Annual peak stages and discharges

\begin{tabular}{|c|c|c|c|c|c|c|c|c|}
\hline $\begin{array}{c}\text { Calendar } \\
\text { year }\end{array}$ & Date & & $\begin{array}{c}\text { Gage } \\
\text { height } \\
\text { (feet) }\end{array}$ & $\begin{array}{c}\text { Discharge } \\
\text { (cfs) }\end{array}$ & $\begin{array}{c}\text { Calendar } \\
\text { year }\end{array}$ & Date & $\begin{array}{c}\text { Gage } \\
\text { height } \\
\text { (feet) }\end{array}$ & $\begin{array}{l}\text { Discharge } \\
\text { (cfs) }\end{array}$ \\
\hline $\begin{array}{l}1936 \\
1937 \\
1938 \\
1939 \\
1940\end{array}$ & $\begin{array}{l}\text { June } \\
\text { Oct. } \\
\text { July } 1 \\
\text { Sept. } 2 \\
\text { Apr. }\end{array}$ & & $\begin{array}{c}4.36 \\
3.98 \\
3.74 \\
- \\
3.45\end{array}$ & $\begin{array}{l}912 \\
673 \\
490 \\
711 \\
484\end{array}$ & $\begin{array}{l}1946 \\
1947 \\
1948 \\
1949 \\
1950\end{array}$ & $\begin{array}{ll}\text { July } & 29 \\
\text { Sept. } & 23 \\
\text { Jan. } & 25 \\
\text { Aug. } & 29 \\
\text { Oct. } & 19\end{array}$ & $\begin{array}{l}4.52 \\
4.82 \\
4.19 \\
4.54 \\
5.40\end{array}$ & $\begin{array}{r}890 \\
1,130 \\
671 \\
929 \\
1,610\end{array}$ \\
\hline $\begin{array}{l}1941 \\
1942 \\
1943 \\
1944 \\
1945\end{array}$ & $\begin{array}{l}\text { July } \\
\text { Sept. } \\
\text { Aug. } \\
\text { Oct. } \\
\text { Sept. }\end{array}$ & $\begin{array}{r}27 \\
7 \\
22 \\
20 \\
17\end{array}$ & $\begin{array}{l}3.84 \\
3.72 \\
4.28 \\
5.08 \\
5.60\end{array}$ & $\begin{array}{r}927 \\
511 \\
884 \\
a 1,580 \\
2,060 \\
\end{array}$ & $\begin{array}{l}1951 \\
1952 \\
1953\end{array}$ & $\begin{array}{lr}\text { Oct. } & 3 \\
\text { Oct. } & 9 \\
\text { Aug. } & 29\end{array}$ & $\begin{array}{l}4.00 \\
4.18 \\
-\end{array}$ & $\begin{array}{r}551 \\
689 \\
a_{1}, 100\end{array}$ \\
\hline
\end{tabular}

a Maximum da1ly. 
St. Johns R1ver Basin

(18) St. Johns River near De Land, Fla.

Location.--Lat $29^{\circ} 01^{\prime}$, long $81^{\circ} 23^{\prime}$, T. 17 S., R. 29 E., on left bank 1,000 ft downstream from Crows Bluff Bridge on State Highway 44 and 5 miles west of DeLand.

Drainage area. $--2,960 \mathrm{sq} \mathrm{ml}$, approximately.

Records avallable.--U. S. Geo:oglcal Survey: January 1934 to December 1953, da1ly gage helghts and discharges.

Gage.--Water-stage recorder. Datum of gage $1 \mathrm{~s} 1.114 \mathrm{ft}$ below mean sea level (levels by Corps of Englneers). Prior to May 3, 1936, staff gage at Crows Bluff Bridge 1,000 ft upstream at same datum.

Aux1llary water-stage: zorder at St. Franc1s Land1ng, 4 miles downstream from Crows Bluff Br1dge. Datum of gage is $.716 \mathrm{ft}$ below mean sea level (levels by Corps of Eng1neers). Prior to Jan. 16, 1943, an add1t: inal aux1l1ary water-stage recorder $1 \frac{1}{2}$ miles upstream from Crows Bluff Brldge.

Stage-d1scharge relation.--Dej ned by current-meter measurements below 17,000 cfs; affected by fall. D1scharge computed $\therefore$ using fall, as determined by auxillary water-stage recorder at St. Francis Landing, as a $f^{\prime}$ ctor.

Remarks.--Slight tide effect $a$. low stages.

4nnual peak stages and discharges

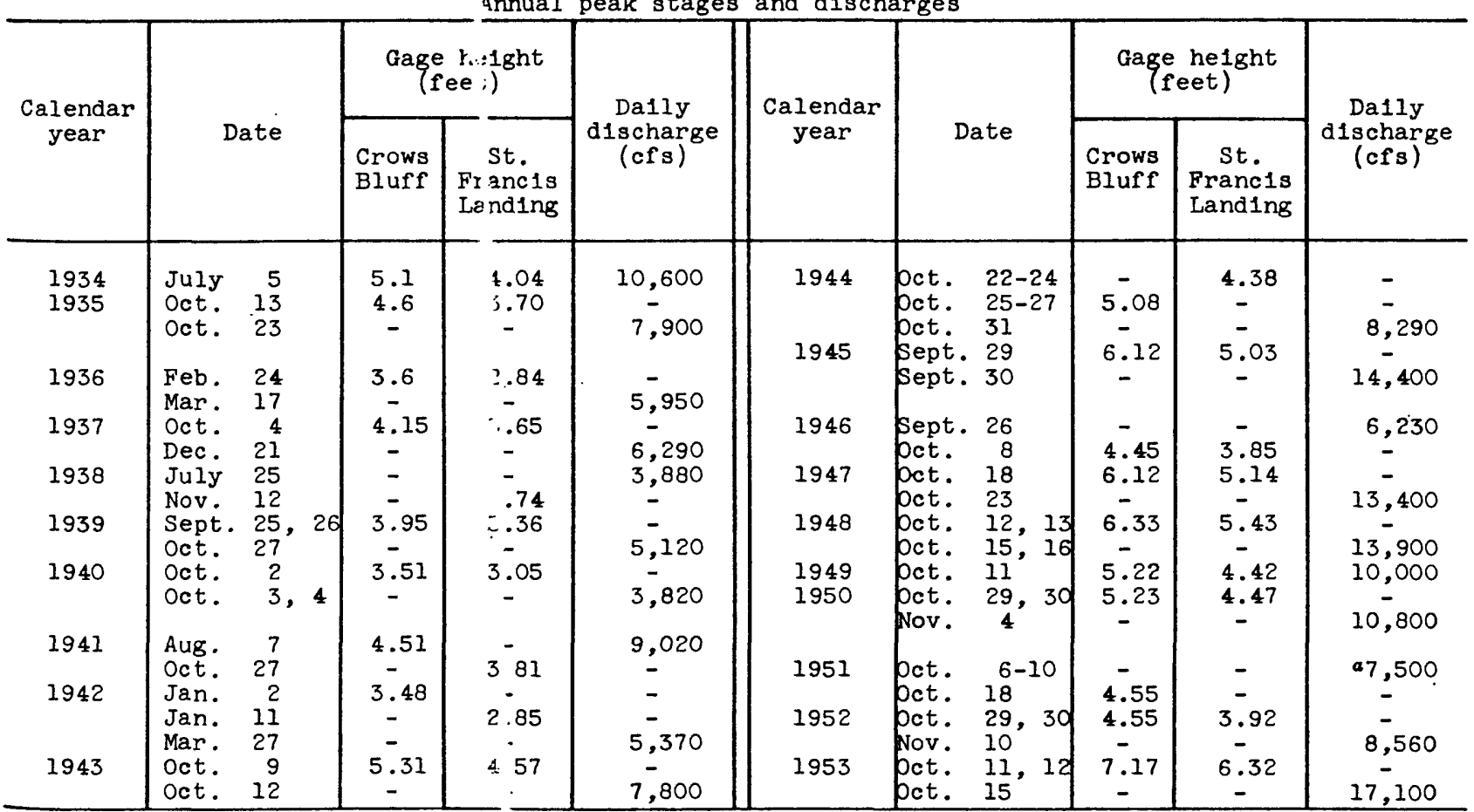

a Estimated average for 5-day period. 
St. Johns R1ver Basin

(19) Lake Apopka at Winter Garden, Fla.

Location.--Lat $28^{\circ} 35^{\prime}$, long $81^{\circ} 35^{\prime}$, in sec. 14, T. $22 \mathrm{~S} ., \mathrm{R} .27 \mathrm{E}$., in southeast corner or west boat basin in c1ty park at W1nter Garden.

Drainage area above outlet. --130 sq $\mathrm{ml}$, approximately.

Records avallable.--Corps of Engineers: December 1935 to December 1942, once monthly gage he1ghts. U. S. Geological Survey: September 1942 to December 1953, da1ly gage helghts.

Gage.--Water-stage recorder. Datum of gage is $62.57 \mathrm{ft}$ above mean sea level, datum of 1929. Prior to Sept. 19, 1942, Corps of Engineers staff gage on clty dock at datum approximately $0.3 \mathrm{ft}$ higher. Gage heights shown below have been reduced to elevations above mean sea level.

Annual peak stages

\begin{tabular}{|c|c|c|c|c|c|}
\hline Calendar year & Date & $\begin{array}{c}\text { Gage height } \\
\text { feet) }\end{array}$ & Calendar year & Date & $\begin{array}{c}\text { Gage height } \\
(\text { feet) }\end{array}$ \\
\hline $\begin{array}{l}1936 \\
1937 \\
1938 \\
1939 \\
1940\end{array}$ & $\begin{array}{l}\text { oct. } \quad 12 \\
\text { Jan. } \quad 10 \\
\text { Oct. } 2 \\
\end{array}$ & $\begin{array}{l}a 69.3 \\
a 68.7 \\
a \quad 68.1 \\
a 67.2 \\
a 67.1\end{array}$ & $\begin{array}{l}1946 \\
1947 \\
1948 \\
1949 \\
1950\end{array}$ & $\begin{array}{l}\text { Sept. } 27,30 \\
\text { Sept. } 27 \\
\text { Jan. } 31 \\
\text { Jan. } 1 \\
\text { Oct. } 24\end{array}$ & $\begin{array}{l}67.59 \\
68.90 \\
68.71 \\
67.09 \\
66.56\end{array}$ \\
\hline $\begin{array}{l}1941 \\
1942 \\
1943 \\
1944 \\
1945 \\
\end{array}$ & $\begin{array}{lr}\text { Dec. } & 1 \\
\text { Mar. } & 3 \\
\text { Sept. } & 23 \\
\text { Oct. } & 24 \\
\text { Sept. } & 20 \\
\end{array}$ & $\begin{array}{r}a 67.6 \\
a^{a} 68.2 \\
67.49 \\
67.83 \\
67.94 \\
\end{array}$ & $\begin{array}{l}1951 \\
1952 \\
1953\end{array}$ & $\begin{array}{c}\text { Feb. 8, Dec. } 27 \\
\text { Feb. } 27 \\
\text { oct. } 9\end{array}$ & $\begin{array}{l}66.47 \\
66.74 \\
68.32\end{array}$ \\
\hline
\end{tabular}

Maximum observed monthly reading; probably not maximum during year.

Note.--Momentary peak stages are wind affected and elevations shown for years 1943 to 1953 are maximum dally flgures.

(20) Apopka-Beauclair Canal near Astatula, Fla.


control and bridge on State Highway 448, $1 \frac{1}{2}$ miles west of Lake Jem, $3 \frac{1}{2}$ miles northeast of Astatula and 5 miles southwest of Mount Dora.

Upper gage is $280 \mathrm{ft}$ upstream from bridge and $330 \mathrm{ft}$ upstream from control. Lower gage 1s $330 \mathrm{ft}$ downstream from control.

Records available.--U. S. Geological Survey: July 1942 to July 1948, da1ly gage he1ghts and periodic discharge measurements prior to the construction of the timber control (completed about Aug. 15, 1950). November 1950 to December 1953, fragmentary gage helghts only, from upper and lower gages.

Gage.--Staff gages at upper and lower locations read once da1ly when an observer is avallable. Datum of each gage is at mean sea level, datum of 1929. Prior to July 1948, before control was constructed, staff gage at approximately same site as upper gage. Gage was read twice dally. Datum of this gage was $60.68 \mathrm{ft}$ above mean sea level, datum of 1929.

Stage-discharge relation.--Not defined.

Historical data.--High water mark on bridge for some flood prior to July 1942 (date unknown) was at elevation $67.0 \mathrm{ft}$. 
St. Johns River Basin

(20) Apopka-Beauclair Canal near Astatula, Fla.--Continued

Annual peak stages

\begin{tabular}{|c|c|c|c|}
\hline \multirow[t]{2}{*}{ Calendar year } & \multirow[t]{2}{*}{ Date } & \multicolumn{2}{|c|}{$\begin{array}{c}\text { Elevation } \\
\text { (feet above } \\
\text { mean sea level) }\end{array}$} \\
\hline & & Former gage & - \\
\hline $\begin{array}{l}1943 \\
1944 \\
1945 \\
1946 \\
1947 \\
1948\end{array}$ & $\begin{array}{ll}\text { Mar. } & 7, \text { Apr. } 19 \\
\text { Oct. } 20 \\
\text { Sept. } 16 \\
\text { Jan. } 16 \\
\text { Sept. } 24 \\
\text { Mar. } \quad 6,12 \\
\end{array}$ & $\begin{array}{l}67.0 \\
65.07 \\
65.73 \\
65.71 \\
65.13 \\
65.18 \\
65.99 \\
\end{array}$ & \\
\hline \multicolumn{4}{|c|}{50} \\
\hline & & Upper gage & Lower gage \\
\hline $\begin{array}{l}1951 \\
1952 \\
1953\end{array}$ & $\begin{array}{lr}\text { Jan. } & 1 \\
\text { Feb. } & 5 \\
\text { Apr. } & 14 \\
\text { Dec. } & 23\end{array}$ & $\begin{array}{r}65.85 \\
66.55 \\
66.78\end{array}$ & $\begin{array}{c}64.13 \\
- \\
64.17 \\
65.78\end{array}$ \\
\hline
\end{tabular}

a Maximum observed during period of fragmentary gagehelght record; probably not maximum during year.

(21) Lake Dora at Mount Dora, Fla.

Location.--Lat $28^{\circ} 48^{\prime}$, long $81^{\circ} 38^{\prime}$, near boundary between secs. 31 and 32 , T. 19 S., R. 27 E., at boat dock in Elizabeth Evans Park in Mount Dora.

Records avallable.--Corps of Englneers: November 1935 to July 1942, gage helghts read monthly or oftener. U. S. Geological Survey: July 1942 to December 1953, dally gage helghts.

Gage.--Staff gage read once da1ly. Datum of gage 1s at mean sea level, datum of 1929. Pr1or to Nov. 17, 1950, datum of gage was $59.88 \mathrm{ft}$ above mean sea level, datum of 1929.

Prior to July 14, 1942, staff gage in boat slip at W1se Boat Works at datum about $0.08 \mathrm{ft}$ h1gher.

H1storlcal data.--H1gh-water of September 1926 reached a stage of $65.8 \mathrm{ft}$ above mean sea level, from Information by Mr. W1se, owner of Wise Boat Works, and Mr. Trema1n, Mount Dora realtor.

Annual peak stages

\begin{tabular}{|c|c|c|c|c|c|}
\hline Calendar year & Date & $\begin{array}{c}\text { Elevation, } \\
\text { feet above } \\
\text { mean sea level }\end{array}$ & Calendar year & Date & $\begin{array}{c}\text { Elevation, } \\
\text { feet above } \\
\text { mean sea level }\end{array}$ \\
\hline 1926 & Sept. - & 65.8 & 1945 & oct. 2,5 & 64.90 \\
\hline $\begin{array}{l}1936 \\
1937 \\
1938 \\
1939 \\
1940\end{array}$ & $\begin{array}{l}\text { Oct. } \overline{-} \\
\text { Oct. -, Dec. - } \\
\text { Jan. } \\
\text { Oct. } \\
\text { Sept. - Oct.- }\end{array}$ & $\begin{array}{l}a_{6} 64.1 \\
a_{64} .2 \\
a_{64.3} \\
a_{62.3} \\
a_{62.3}\end{array}$ & $\begin{array}{l}1946 \\
1947 \\
1948 \\
1949 \\
1950\end{array}$ & $\begin{array}{l}\text { Jan. } 17 \\
\text { Nov. } 25,26 \\
\text { Oct. } 6 \\
\text { Oct. } 5 \\
\text { Jan. } 1\end{array}$ & $\begin{array}{l}64.50 \\
63.96 \\
65.21 \\
65.22 \\
64.5\end{array}$ \\
\hline $\begin{array}{l}1941 \\
1942 \\
1943 \\
1944 \\
\end{array}$ & $\begin{array}{l}\text { Aug. }- \\
\text { Jan. Apr. - } \\
\text { Sept. } 22 \\
\text { Nov. } 5,6,10\end{array}$ & $\begin{array}{c}a 63.6 \\
a 63.6 \\
63.18 \\
64.28 \\
\end{array}$ & $\begin{array}{l}1951 \\
1952 \\
1953\end{array}$ & $\begin{array}{l}\text { Jan. } 8,15,16 \\
\text { Apr. } 1,2 \\
\text { Dec. } 28-31\end{array}$ & $\begin{array}{l}63.82 \\
63.70 \\
65.36\end{array}$ \\
\hline
\end{tabular}

a Maximum observed monthly reading; probably not maximum during year. 


\section{St. Johns River Basin}

(22) Dead River near Tavares, Fla.

Location.--Lat $28^{\circ} 49^{\prime}$, long $81^{\circ} 45^{\prime}$, near corner of secs. 24 and 25 of R. 25 E., and 19 and 30 of R. 26 E., T. $19 \mathrm{~S}$., on right bank $15 \mathrm{ft}$ upstream from bridge on U. S. Highway 441 , and $2 \mathrm{mlles}$ west of Tavares.

Records ava1lable.--U. S. Geological Survey: July 1942 to December 1953, da1ly gage he1ghts. Gage.--Staff gage read once da1ly. Datum of gage 1s $59.30 \mathrm{ft}$ above mean sea level, datum of 1929.

Stage-discharge relation.--Not defined. The normal flow in Dead River is in northeasterly direction or from Lake Harris to Lake Eust1s, but at times during occurrence of strong northeast winds, the flow has been observed to reverse, or from Lake Eustis to Lake Harris.

H1storlcal data.--H1gh-water of 1926 reached a stage of $66.1 \mathrm{ft}$ above mean sea level, from Information by Mr. T. W. Camp, Seaboard Rallway station agent.

Annual peak stages

\begin{tabular}{|c|c|c|c|c|c|}
\hline Calendar year & Date & $\begin{array}{c}\text { Elevation, } \\
\text { feet above } \\
\text { mean sea level }\end{array}$ & Calendar year & Date & $\begin{array}{c}\text { Elevation, } \\
\text { feet. above } \\
\text { mean sea level }\end{array}$ \\
\hline $\begin{array}{l}1926 \\
1943 \\
1944 \\
1945 \\
1946 \\
1947\end{array}$ & $\begin{array}{l}- \\
\text { Sept. } 20,23,24 \\
\text { Oct. } 23 \\
\text { Oct. } 3-7,10-14 \\
\text { Jan. } \quad 1 \\
\text { Nov. } 4-17\end{array}$ & $\begin{array}{l}66.1 \\
62.26 \\
63.20 \\
63.98 \\
63.68 \\
63.36 \\
\end{array}$ & $\begin{array}{l}1948 \\
1949 \\
1950 \\
1951 \\
1952 \\
1953\end{array}$ & $\begin{array}{l}\text { Oct. } \quad 8,9 \\
\text { Oct. } 8,9,15,16 \\
\text { Jan. } 1,2 \\
\text { Jan. } 5-7 \\
\text { Mar. } 29-\text { Apr. } 2 \\
\text { Dec. } 31\end{array}$ & $\begin{array}{l}64.08 \\
64.50 \\
63.76 \\
\\
63.10 \\
62.70 \\
64.52\end{array}$ \\
\hline
\end{tabular}

(23) Lake Minnehaha at clermont, Fla.

Location.--Lat $28^{\circ} 33^{\prime}$, long $81^{\circ} 45^{\prime}$, in sec. 30, T. 22 S., R. 26 E., on north shore, 96 ft south of centerline of Lake Shore Drive between East Avenue and Drew Ávenue in Clermont.

Records ava1lable.--U. S. Geological Survey: May 1945 to December 1953, da1ly gage he1ghts.

Gage.-Water-stage recorder. Datum of gage $1 \mathrm{~s} 91.32 \mathrm{ft}$ above mean sea level, datum of 1929.

Prior to June 5,1946 , staff gage at same site and datum read once da1ly.

Annual peak stages

\begin{tabular}{|c|c|c|c|c|c|}
\hline Calendar year & Date & $\begin{array}{l}\text { Gage height } \\
\text { (feet) }\end{array}$ & Calendar year & Date & $\begin{array}{l}\text { Gage helght } \\
\text { feet) }\end{array}$ \\
\hline 1945 & Sept. 24 to Oct. 4 & 7.50 & 1950 & oct. $26-28$ & 6.00 \\
\hline $\begin{array}{l}1946 \\
1947 \\
1948 \\
1949 \\
\end{array}$ & $\begin{array}{lr}\text { Oct. } 8 \\
\text { Oct. } 6,7 \\
\text { Oct. } 13-15 \\
\text { Oct. } 11-13 \\
\end{array}$ & $\begin{array}{l}5.90 \\
6.96 \\
6.98 \\
6.72 \\
\end{array}$ & $\begin{array}{l}1951 \\
1952 \\
1953\end{array}$ & $\begin{array}{l}\text { Dec. } 1 \\
\text { Jan. } \\
\text { Oct. } \\
\end{array}$ & $\begin{array}{l}6.36 \\
5.6 \\
7.52\end{array}$ \\
\hline
\end{tabular}




\section{St. Johns R1ver Bas1n}

(24) Palatlakaha Creek near Mascotte, Fla.

Location.--Lat $28^{\circ} 37^{\prime}$, long $81^{\circ} 51^{\prime}$, in sec. 36, T. 21 S., R. 24 E., on right bank 5 ft upstream from county h1ghway br1dge, 0.2 m1le downstream from Lake Emma, and $3 \frac{1}{4}$ m1les northeast of Mascotte.

Dra1nage area.--160 sq $\mathrm{ml}$, approximately.

Records ava1lable.--U. S. Geological Survey: May 1945 to December 1953, da1ly gage helghts and discharges.

Gage.--Water-stage recorder. Datum of gage is $89.54 \mathrm{ft}$ above mean sea level, datum of 1929. Prior to May 21, 1946, staff gage at same s1te and datum read once da1ly.

Stage-discharge relation.--Defined by current-meter measurements below 420 cfs. Cons1derable shifting at all stages and backwater effect from hyacinths jams and lakes below station.

\begin{tabular}{|c|c|c|c|c|c|c|c|}
\hline $\begin{array}{c}\text { Calendar } \\
\text { year }\end{array}$ & Date & $\begin{array}{c}\text { Gage } \\
\text { height } \\
\text { (feet) }\end{array}$ & $\begin{array}{c}\text { Discharge } \\
\text { (cfs) }\end{array}$ & $\begin{array}{c}\text { Calendar } \\
\text { year }\end{array}$ & Date & $\begin{array}{l}\text { Gage } \\
\text { height } \\
\text { (feet) }\end{array}$ & $\begin{array}{l}\text { Discharge } \\
\text { (cfs) }\end{array}$ \\
\hline 1945 & oct. 4,5 & 7.06 & 458 & 1950 & oct. 21 & 5.52 & 222 \\
\hline $\begin{array}{l}1946 \\
1947 \\
1948 \\
1949 \\
\end{array}$ & $\begin{array}{ll}\text { Sept. } & 21 \\
\text { Oct. } & 16 \\
\text { Oct. } & 18 \\
\text { Sept. } 29\end{array}$ & $\begin{array}{l}5.20 \\
6.61 \\
6.35 \\
6.31\end{array}$ & $\begin{array}{l}164 \\
362 \\
326 \\
342 \\
\end{array}$ & $\begin{array}{l}1951 \\
1952 \\
1953\end{array}$ & $\begin{array}{l}\text { Dec: } 27 \\
\text { Mar: } 28 \\
\text { Oct: } 11,12\end{array}$ & $\begin{array}{l}5.84 \\
5.50 \\
7.12\end{array}$ & $\begin{array}{l}206 \\
152 \\
372\end{array}$ \\
\hline
\end{tabular}

(25) Haines Creek at Lisbon, Fla.

Location.--Lat $28^{\circ} 53^{\prime}$, long $81^{\circ} 47$, in sec. 2, T. $19 \mathrm{~S} .$, R. $25 \mathrm{E}$., on left bank 15 ft downstream from bridge on State Highway 44 , a quarter of a m1le west of Lisbon, and $8 \frac{1}{2}$ miles northeast of Leesburg.

Drainage area. $--640 \mathrm{sq} \mathrm{m}$, approximately.

Records avallable.--U. S. Geological Survey: July 1942 to December 1953, da1ly gage helghts and discharges.

Gage.--Staff gage read once da1ly. Datum of gage is $59.22 \mathrm{ft}$ above mean sea level, datum of 1929 (Corps of Englneers benchmark).

Stage-discharge relation.--Defined by current-meter measurements below 805 cf's. Considerable shifting at all stages.

Historical data.--Flood of 1926 reached a stage about $6.4 \mathrm{ft}$, from information by Mr. George $R$. Gastfleld, observer.

Annual peak stages and discharges

\begin{tabular}{c|c|c|c||c|c|c|c}
\hline $\begin{array}{c}\text { Calendar } \\
\text { year }\end{array}$ & Date & $\begin{array}{c}\text { Gage } \\
\text { height } \\
\text { (feet) }\end{array}$ & $\begin{array}{c}\text { Discharge } \\
\text { (cfs) }\end{array}$ & $\begin{array}{c}\text { Calendar } \\
\text { year }\end{array}$ & Date & $\begin{array}{c}\text { Gage } \\
\text { height } \\
\text { (feet) }\end{array}$ & $\begin{array}{c}\text { Discharge } \\
\text { (cfs) }\end{array}$ \\
\hline 1926 & - & 6.4 & - & 1948 & Oct. 5, 9, 10 & 3.84 & 557 \\
1943 & Sept. 21-23 & 2.51 & 196 & 1949 & Oct. 7, 8, 11 & 4.22 & 704 \\
1944 & Oct. 26 & 3.16 & 384 & 1950 & Oct. 29 & 3.04 & 361 \\
1945 & Oct. 26 & 3.68 & 585 & 1951 & Feb. 7 & 3.11 & 382 \\
1946 & Feb. 28 & 3.39 & 489 & 1952 & Mar. 31 to Apr. & 2.77 & 400 \\
1947 & Nov. 12 & 3.24 & 429 & & Dec. 29 & 4.20 & 805 \\
\hline
\end{tabular}




\section{St. Johns River Basin}

(26) Lake Weir at Oklawahe, Fla.

Location.--Lat $29^{\circ} 02^{\prime} 30^{\prime \prime}$, long $81^{\circ} 55^{\prime} 30^{\prime \prime}$, in sec. 5, T. $17 \mathrm{~S} ., \mathrm{R} .24 \mathrm{E}$. , on dock $100 \mathrm{ft}$ east of Johnsons Beach Pavilion, 0.2 mile south of Post Office in Oklawaha.

Drainage area above outlet. $--37 \mathrm{sq} \mathrm{ml}$, approximately.

Records avallable.--Corps of Englneers: Apr11 1936 to December 1943, once monthly gage helghts. U. S. Geological Survey: November 1942 to December 1953, daily gage helghts and occasional discharge measurements of flow at the outlet.

Gage.--Water-stage recorder. Datum of gage $1 \mathrm{~s} 55.58 \mathrm{ft}$ above mean sea level (Corps of Eng1neers benchmark). Prior to Nov. 5, 1942, Corps of Engineers staff gage at approximately same site at datum at mean sea level. (All gage helghts shown below have been reduced to elevations above mean sea level).

Annual peak stages

\begin{tabular}{|c|c|c|c|c|c|}
\hline Calendar year & Date & $\begin{array}{c}\text { Elevation, } \\
\text { feet above } \\
\text { mean sea level }\end{array}$ & Calendar year & Date & $\begin{array}{c}\text { Elevation, } \\
\text { feet above } \\
\text { mean sea level }\end{array}$ \\
\hline $\begin{array}{l}1936 \\
1937 \\
1938 \\
1939 \\
1940\end{array}$ & $\begin{array}{l}\text { Apr. -, Oct. - } \\
\text { Sept.-,oct.-,Dec.- } \\
\text { Jan. - } \\
\text { Oct. - } \\
\text { Aug. - }\end{array}$ & $\begin{array}{l}59.0 \\
a 59.5 \\
+59.6 \\
=58.4 \\
558.1\end{array}$ & $\begin{array}{l}1946 \\
1947 \\
1948 \\
1949 \\
1950\end{array}$ & $\begin{array}{l}\text { Mar. } 14,15,28,29 \\
\text { Oct. } 24 \\
\text { Oct. } 5 \\
\text { Oct. } 1,2 \\
\text { Oct. } 19\end{array}$ & $\begin{array}{l}58.02 \\
58.44 \\
58.34 \\
58.44 \\
57.83\end{array}$ \\
\hline $\begin{array}{l}1941 \\
1942 \\
1943 \\
1944 \\
1945\end{array}$ & $\begin{array}{lr}\text { Aug. } & - \\
\text { Jan. } & - \\
\text { Sept. } & 22 \\
\text { Oct. } & 20 \\
\text { Sept. } & 17\end{array}$ & $\begin{array}{l}558.4 \\
58.6 \\
57.63 \\
57.76 \\
57.78\end{array}$ & $\begin{array}{l}1951 \\
1952 \\
1953\end{array}$ & $\begin{array}{l}\text { Jan. } \quad \text { 4-7 } \\
\text { Mar. } 27,28 \\
\text { Dec. } 25,26,29-31\end{array}$ & $\begin{array}{l}57.49 \\
57.71 \\
58.11\end{array}$ \\
\hline
\end{tabular}

a Maximum observed monthly reading; probably not maximum during year.

Note.--Momentary peak stages are wind affected and elevat1ons shown for years 1943 to 1953 are maximum da1ly figures.

(27) Oklawahe River at Moss Bluff, Fla.

Location.--Lat $29^{\circ} 05^{\prime}$, long $81^{\circ} 53^{\prime}$, in sec. 22 or $23, T .16 \mathrm{~S} ., \mathrm{R}, 24 \mathrm{E}$, on left bank $25 \mathrm{ft}$ upstream from old channel, 50 ft upstream from h1ghway bridge, $600 \mathrm{ft}$ downstream from powerplant, and $0.4 \mathrm{mile}$ southwest of Moss Bluff.

Drainage area. $--910 \mathrm{sq} \mathrm{ml}$, approximately.

Records avaliable.--U. S. Geolog1cal Survey: February to September 1943, occas1onal discharge measurements. October 1943 to December 1953, da1ly gage helghts and discharges.

Gage.--Water-stage recorder. Datum of gage is at mean sea level (Corps of Englneers benchmark). Prior to Aug. 12, 1943, staff gage at same site and datum.

Stage-discharge relation.--Def1ned by current-meter measurements below 1,050 cfs. Cons1derable shifting at all stages.

Remarks.--Records Include flow of old Oklawaha R1ver channel. Flow regulated by powerplant above station. 
St. Johns River Basin

(27) Oklawaha River at Moss Bluff, Fla.--Continued

Annual peak stages and discharges

\begin{tabular}{|c|c|c|c|c|c|c|c|}
\hline $\begin{array}{c}\text { Calendar } \\
\text { year }\end{array}$ & Date & $\begin{array}{l}\text { Gage } \\
\text { height } \\
\text { (feet) }\end{array}$ & $\begin{array}{l}\text { Discharge } \\
\text { (cfs) }\end{array}$ & $\begin{array}{c}\text { Calendar } \\
\text { year }\end{array}$ & Date & $\begin{array}{l}\text { Gage } \\
\text { height } \\
\text { (feet) }\end{array}$ & $\begin{array}{l}\text { Discharge } \\
\text { (cfs) }\end{array}$ \\
\hline $\begin{array}{l}1944 \\
1945\end{array}$ & $\begin{array}{ll}\text { Oct. } & 1 \\
\text { Aug. } & 2\end{array}$ & $\begin{array}{l}49.31 \\
48.90\end{array}$ & $\begin{array}{l}988 \\
910\end{array}$ & 1950 & Oct. 19 & 48.10 & \\
\hline $\begin{array}{l}1946 \\
1947 \\
1948 \\
1949\end{array}$ & $\begin{array}{ll}\text { Feb. } & 2 \\
\text { Sept. } & \\
\text { Aug. } & 1 \\
\text { Aug. } & 2 \\
\text { Sept. } & 2\end{array}$ & $\begin{array}{c}48.37 \\
48.50 \\
48.42 \\
48.47 \\
-\end{array}$ & $\begin{array}{c}811 \\
831 \\
811 \\
- \\
855\end{array}$ & $\begin{array}{l}1951 \\
1952 \\
1953\end{array}$ & $\begin{array}{l}\text { Feb. } 7 \\
\text { Feb. } 17 \\
\text { Dec. } 28\end{array}$ & $\begin{array}{l}46.41 \\
47.94 \\
49.20\end{array}$ & $\begin{array}{r}555 \\
731 \\
1,020\end{array}$ \\
\hline
\end{tabular}

(28) Oklawaha River near Ocala, Fla.

Location.--Lat $29^{\circ} 11^{\prime}$, long $82^{\circ} 00^{\prime}$, in sec. 15, T. $15 \mathrm{~S} ., \mathrm{R} .23 \mathrm{E}$, , on left bank about $15 \mathrm{ft}$ upstream from county highway bridge known as Sharpes Ferry, 2 miles upstream from Silver River, and 9 miles east of Ocala.

Drainage aréa. $--1,100 \mathrm{sq} \mathrm{m}$, approximately.

Records ava1lable.--U. S. Geological Survey: February 1930 to December 1953, da1ly gage helghts and discharges.

Gage.--Water-stage recorder. Datum of gage is $36.52 \mathrm{ft}$ above mean sea level, datum of 1929, supplementary adjustment of 1937 (Corps of Engineers benchmark). Prior to Mar. 2, 1932, staff gage at same site and datum.

Stage-discharge relation.--Defined by current-meter measurements below 1,400 cfs. Considerable shifting at all stages.

Annual peak stages and discharges




St. Johns R1ver Basin

(29) S1lver Springs near Ocala, Fla.

Location.--Measuring point for discharge measurements at lat $29,13^{\prime}$, long $82^{\circ} 02^{\prime}$, in sec. 6 , T. 15 S., R. 23 E., $700 \mathrm{ft}$ downstream from Paradise Landing, $0.7 \mathrm{mlle}$ downstream from head of springs and 6.0 miles northeast of Ocala.

Records ava1lable.--U. S. Geological Survey: January 1933 to December 1953, da1ly or weekly gage helghts and da1ly discharges.

Gage.--Water-stage recorder at head of springs in boat repair basin. Datum of gage is $38.96 \mathrm{ft}$ above mean sea level, datum of 1929. Prlor to Feb. 20, 1947, staff gage at same site and datum.

Auxillary water-stage recorder on Sharpes Ferry artesian well about 400 ft east of Oklawaha River, 2 miles upstream from Silver River, and 4.2 miles southeast of head of springs. Datum of gage 1s $42.36 \mathrm{ft}$ above mean sea level, datum of 1929, supplementary adjustment of 1937 (Corps of Englneers benchmark).

Stage-discharge relation.--No relation between stage at head of springs and discharge of springs becuase of backwater effect from Oklawaha River. Discharge computed from relation between artesian pressure at Sharpes Ferry well and discharge at measuring point. Th1s relation is well defined by current-meter measurements and minor shifts have occurred.

Annual peak stages and discharges






\section{St. Johns River Basin}

(30) Oklawaha River near Conner, Fla.

Location.--Lat $29^{\circ} 13^{\prime}$, long $81^{\circ} 59^{\prime}$, in sec. 3, T. $15 \mathrm{~S} ., \mathrm{R} .23 \mathrm{E}$, on right bank about $15 \mathrm{ft}$ downstream from bridge on State Highway 40, a quarter of a mile downstream from Silver River, about $1 \frac{1}{2}$ miles southwest of Connor, and $8 \mathrm{miles}$ east of Ocala.

Dra1nage area. $--1,180 \mathrm{sq} \mathrm{ml}$, approximately.

Records avallable.--U. S. Geolog1cal Survey: February 1930 to September 1946, da1ly gage helghts and discharges.

Gage.-Water-stage recorder. Datum of gage $1 \mathrm{~s} 31.79 \mathrm{ft}$ above mean sea level, datum of 1929. Feb. 13, 1930, to Aug. 23, 1935, staff gage and Aug. 24, 1935, to June 11, 1939, water-stage recorder at upstream s1de of bridge at same datum.

Stage-discharge relation.--Defined by current-meter measurements below 3,630 cfs. Cons1derable shifting at all stages.

Annual peak stages and discharges

\begin{tabular}{|c|c|c|c|c|c|c|c|}
\hline $\begin{array}{l}\text { Calendar } \\
\text { year }\end{array}$ & Date & $\begin{array}{c}\text { Gage } \\
\text { height } \\
\text { (feet) }\end{array}$ & $\begin{array}{l}\text { Discharge } \\
\text { (cfs) }\end{array}$ & $\begin{array}{l}\text { Calendar } \\
\text { year }\end{array}$ & Date & $\begin{array}{c}\text { Gage } \\
\text { height } \\
\text { (feet) }\end{array}$ & $\begin{array}{l}\text { Discharge } \\
\text { (cfs) }\end{array}$ \\
\hline 1930 & Mar. 2 & 7.37 & 2,280 & $\begin{array}{l}1939 \\
1940\end{array}$ & $\begin{array}{ll}\text { Aug. } & 30 \\
\text { Feb. } & 19,20\end{array}$ & $\begin{array}{l}6.28 \\
5.41\end{array}$ & $\begin{array}{l}1,720 \\
1,290\end{array}$ \\
\hline $\begin{array}{l}1931 \\
1932 \\
1933 \\
1934 \\
1935\end{array}$ & $\begin{array}{l}\text { Apr. } \\
\text { June } 1 \\
\text { Sept. } \\
\text { June } 1 \\
\text { Sept. }\end{array}$ & $\begin{array}{l}7.29 \\
4.69 \\
9.14 \\
8.50 \\
6.65\end{array}$ & $\begin{array}{r}2,430 \\
945 \\
3,700 \\
3,430 \\
1,860\end{array}$ & $\begin{array}{l}1941 \\
1942 \\
1943 \\
1944 \\
1945\end{array}$ & $\begin{array}{ll}\text { Apr. } & 5 \\
\text { July } & 27 \\
\text { Aug. } 21, & \text { Sept. } 22 \\
\text { Oct. } 20 \\
\text { Sept. } & 17\end{array}$ & $\begin{array}{l}- \\
6.67 \\
- \\
7.33 \\
7.14\end{array}$ & $\begin{array}{l}1,460 \\
1,890 \\
1,450 \\
2,320 \\
2,190\end{array}$ \\
\hline $\begin{array}{l}1936 \\
1937 \\
1938\end{array}$ & $\begin{array}{l}\text { June } \\
\text { Aug. } 3 \\
\text { July }\end{array}$ & $\begin{array}{l}6.78 \\
7.58 \\
6.50\end{array}$ & $\begin{array}{l}1,860 \\
2,590 \\
1,680\end{array}$ & 1946 & Feb. 28 & 6.86 & 2,120 \\
\hline
\end{tabular}

(31) Oklawaha River at Eureka, Fla.

Location.--Lat $29^{\circ} 22^{\prime}$, long $81^{\circ} 54^{\prime}$, in sec. 9, T. 13 S., R. 24 E., on right bank 20 ft upstream from bridge on State Highway 316 in Eureka, and 3 miles downstream from Eaton Creek.

Drainage area.--1,420 sq $\mathrm{ml}$, approximately.

Records avallable.--U. S. Geological Survey: February 1930 to June 1934, September 1943 to December 1952, da1ly gage helghts and discharges.

Gage.--Water-stage recorder. Datum of gage is $15.44 \mathrm{ft}$ above mean sea level, datum of 1929 (Corps of Englneers benchmark). Prior to Nov. 19, 1943, staff gage at same site and datum.

Stage-discharge relation.--Defined by current-meter measurements below 2,800 cfs. Considerable shifting at all stages. 
St. Johns River Basin

(31) Oklawaha R1ver at Eureka, Fla.--Continued

Annual peak stages and discharges

\begin{tabular}{|c|c|c|c|c|c|c|c|}
\hline $\begin{array}{c}\text { Calendar } \\
\text { year }\end{array}$ & Date & $\begin{array}{l}\text { Gage } \\
\text { height } \\
\text { (feet) }\end{array}$ & $\begin{array}{c}\text { Discharge } \\
\text { (cfs) }\end{array}$ & $\begin{array}{c}\text { Calendar } \\
\text { year }\end{array}$ & Date & $\begin{array}{c}\text { Gage } \\
\text { height } \\
\text { (feet) }\end{array}$ & $\begin{array}{c}\text { Discharge } \\
\text { (cfs) }\end{array}$ \\
\hline $\begin{array}{l}1930 \\
1931 \\
1932 \\
1933 \\
1934 \\
1943 \\
1944 \\
1945\end{array}$ & $\begin{array}{lr}\text { Mar. } & 31 \\
& \text { Apr. } \\
\text { June } & 20 \\
\text { Sept. } & 7 \\
\text { June } & 17 \\
& \\
\text { Sept. } & 22 \\
\text { Oct. } & 21 \\
\text { Sept. } & 18 \\
\end{array}$ & $\begin{array}{l}8.02 \\
7.88 \\
5.13 \\
11.0 \\
10.06 \\
7.00 \\
8.75 \\
8.42 \\
\end{array}$ & $\begin{array}{l}3,400 \\
3,030 \\
1,080 \\
6,260 \\
5,360 \\
2,220 \\
3,950 \\
3,520 \\
\end{array}$ & $\begin{array}{l}1946 \\
1947 \\
1948 \\
1949 \\
1950 \\
1951 \\
1952\end{array}$ & $\begin{array}{lr}\text { Mar. } & 2 \\
\text { Sept. } & 25 \\
\text { Oct. } & 5 \\
\text { Aug. } & 30 \\
\text { Sept. } & 7 \\
& \\
\text { Sept. } & 21 \\
\text { Feb. } & 19\end{array}$ & $\begin{array}{r}7.60 \\
7.81 \\
7.52 \\
8.03 \\
10.12 \\
6.74 \\
7.16\end{array}$ & $\begin{array}{l}2,760 \\
2,940 \\
2,750 \\
3,230 \\
5,370 \\
2,120 \\
2,460\end{array}$ \\
\hline
\end{tabular}

(32) Newnans Lake near Galnesville, Fla.

Location.--Lat $29^{\circ} 39^{\prime}$, long $82^{\circ} 14^{\prime}$, in W $\frac{1}{2}$ sec. 5, T. $10 \mathrm{~S}$, , R. 21 E., on west shore of lake at Myers fish camp, 5 miles east of Gainesville.

Records ava1lable.--Corps of Eng1neers: Apr1l 1936 to December 1947, once monthly gage he1ghts. U. S. Geological Survey: November 1945 to September 1952, twice weekly gage helghts.

Gage.--Staff gage. Datum of gage $1863.33 \mathrm{ft}$ above mean sea level, datum of 1929. Pr1or to June 27,1951 , at varlous nearby locations at same datum.

Annual peak stages

\begin{tabular}{|c|c|c|c|c|c|}
\hline Calendar year & Date & $\begin{array}{c}\text { Gage he1ght } \\
\text { (feet) }\end{array}$ & Calendar year & Date & $\begin{array}{l}\text { Gage he1ght } \\
\text { feet) }\end{array}$ \\
\hline $\begin{array}{l}1936 \\
1937 \\
1938 \\
1939 \\
1940 \\
1941 \\
1942 \\
1943 \\
1944 \\
\end{array}$ & $\begin{array}{l}\text { Apr. - } \\
\text { Oct. - } \\
\text { Jan. - } \\
\text { July - } \\
\text { Mar. - } \\
\text { Nov. - } \\
\text { Jan. - } \\
\text { Oct. - } \\
\text { Nov. - }\end{array}$ & $\begin{array}{l}a 3.7 \\
a 5.0 \\
a 4.3 \\
a 5.9 \\
a 4.1 \\
\\
a 5.2 \\
a 4.8 \\
a 2.6 \\
=5.2\end{array}$ & $\begin{array}{l}1945 \\
1946 \\
1947 \\
1948 \\
1949 \\
1950 \\
\\
1951 \\
1952 \\
\end{array}$ & $\begin{array}{lr}\text { Dec. } & 29 \\
& \\
\text { Aug. } & 5 \\
\text { Oct. } & 27 \\
\text { Mar. } & 12 \\
\text { Sept. } & 6 \\
\text { Sept. } & 12 \\
& \\
\text { Nov. } & 23 \\
\text { Mar. } & 4 \\
\end{array}$ & $\begin{array}{l}5.14 \\
6.39 \\
6.64 \\
7.88 \\
5.42 \\
6.18 \\
4.50 \\
4.85\end{array}$ \\
\hline
\end{tabular}

Note.--Readings for years 1945-52 were maximum observed at twice-weekly intervals; peak stage may have been slightly higher. 


\section{St. Johns River Basin}

(33) Orange Lake near Boardman, Fla.

Location.--Lat $29^{\circ} 27^{\prime}$, long $82^{\circ} 11^{\prime}$, In sec. 15, T. 12 S., R. 21 E., on southwest shore of lake, $1 \frac{3}{4} \mathrm{~m} 1 \mathrm{les}$ southeast of Boardman.

Records ava1lable.--Corps of Engineers: November 1935 to December 1947, once monthly gage helghts from gage located in town of Orange Lake. U. S. Geological Survey: November 1945 to May 1948, dally or twice weekly gage heights.

Gage.--Staff gage. Datum of gage is $53.44 \mathrm{ft}$ above mean sea level, datum of 1929 . Datum of Corps of Englneers staff gage at town of Orange Lake was $56.2 \mathrm{ft}$ above mean sea level. Gage helghts collected at the two locations are comparable. (All gage helghts shown below have been reduced to elevations above mean sea level).

Annual peak stages

\begin{tabular}{|c|c|c|c|c|c|}
\hline Calendar year & Date & $\begin{array}{c}\text { Elevation, } \\
\text { feet above } \\
\text { mean sea level }\end{array}$ & Calendar year & Date & $\begin{array}{l}\text { Elevation, } \\
\text { feet above } \\
\text { mean sea level }\end{array}$ \\
\hline $\begin{array}{l}1936 \\
1937 \\
1938 \\
1939 \\
1940 \\
1941 \\
1942\end{array}$ & $\begin{array}{c}\text { Mar. - } \\
\text { Oct. - } \\
\text { Jan. - } \\
\text { Aug. - } \\
\text { May-, Aug.-, Sept.- } \\
\text { Nov. - } \\
\text { Jan. -, Apr. - }\end{array}$ & $\begin{array}{l}a 59.0 \\
a 59.6 \\
a 59.7 \\
a_{60.0} \\
a_{58.2} \\
a_{61} .5 \\
a_{60.1}\end{array}$ & $\begin{array}{l}1943 \\
1944 \\
1945 \\
1946 \\
1947 \\
1948\end{array}$ & $\begin{array}{l}\text { Jan. } \\
\text { Nov. } \\
\text { Oct. } \\
\text { Oct. } 12 \\
\text { Nov. } 19 \\
\text { Mar. } 19\end{array}$ & $\begin{array}{l}a_{57.4} \\
\text { a } 58.4 \\
\text { a59.0 } \\
\\
60.84 \\
60.68 \\
61.62\end{array}$ \\
\hline
\end{tabular}

a Maximum observed monthly reading; probably not maximum during year.

(34) Orange Lake Outlet near Citra, Fla.

Location.--Lat $29^{\circ} 26^{\prime}$, long $82^{\circ} 07^{\prime}$, in sec. 21 , T. $12 \mathrm{~S} ., \mathrm{R} .22 \mathrm{E}$, , on left bank 15 ft upstream from bridge on U. S. Highway 301 and State Highway $200,0.8 \mathrm{mile}$ south of Island Grove, and 1.5 miles north of C1tra.

Drainage area.--Indeterminate. Total drainage area of Orange Lake Outlet and Lochloosa Lake Outlet above highway is $323 \mathrm{sq} \mathrm{mi}$.

Records avallable.--U. S. Geological Survey: November 1941, three discharge measurements; July 1942 to December 1946, dally gage helghts; January 1947 to December 1953, dally gage heights and discharges.

Gage.--Water-stage recorder. Datum of gage is $53.41 \mathrm{ft}$ above mean sea level, datum of 1929. July 2, 1942 , to June 27,1943 , staff gage at same site and at datum 2.00 ft higher. June 28 , 1943 , to Mar. 27, 1947, staff gage and same site and datum.

Stage-discharge relation.--Defined by current-meter measurements for period 1947 to 1953. Shifting channel and channel improvement work preclude computation of discharge maxima for other years.

Historical data.--Flood of October 1941 reached a stage of $7.7 \mathrm{ft}$, from floodmarks on bridge.

Remarks.--Orange and Lochloosa are connected by Cross Creek through which there may be a natural diversion from one lake to the other. 
St. Johns River Basin

(34) Orange Lake Outlet near C1tra, Fla.--Continued

Annual peak stages and discharges

\begin{tabular}{|c|c|c|c|c|c|c|c|}
\hline $\begin{array}{c}\text { Calendar } \\
\text { year }\end{array}$ & Date & $\begin{array}{l}\text { Gage } \\
\text { height } \\
\text { (feet) }\end{array}$ & $\begin{array}{l}\text { Discharge } \\
\text { (cfs) }\end{array}$ & $\begin{array}{l}\text { Calendar } \\
\text { year }\end{array}$ & Date & $\begin{array}{l}\text { Gage } \\
\text { height } \\
\text { (feet) }\end{array}$ & $\begin{array}{l}\text { Discharge } \\
\text { (cfs) }\end{array}$ \\
\hline 1941 & October & a 7.7 & - & 1947 & Nov. 18 & 7.06 & 417 \\
\hline $\begin{array}{l}1943 \\
1944 \\
1945\end{array}$ & $\begin{array}{l}\text { Jan. } 1,2 \\
\text { Nov. } 11 \\
\text { Sept. } 17\end{array}$ & $\begin{array}{r}3.97 \\
4.92 \\
5.60\end{array}$ & $\overline{-}$ & $\begin{array}{l}1949 \\
1950\end{array}$ & $\begin{array}{l}\text { Oct. } 12-14 \\
\text { Oct. } 27\end{array}$ & $\begin{array}{l}5.63 \\
6.06\end{array}$ & $\begin{array}{l}139 \\
401\end{array}$ \\
\hline 1946 & $\begin{array}{l}\text { Aug. } 15, \\
\text { Oct. } 11-14\end{array}$ & 7.30 & - & $\begin{array}{l}1951 \\
1952 \\
1953 \\
\end{array}$ & $\begin{array}{ll}\text { Dec. } & 27 \\
\text { Feb. } & 27 \\
\text { Oct. } & 10 \\
\end{array}$ & $\begin{array}{l}4.98 \\
5.44 \\
6.26 \\
\end{array}$ & $\begin{array}{l}173 \\
251 \\
467 \\
\end{array}$ \\
\hline
\end{tabular}

a Present datum.

(35) Lochloosa Lake at Lochloosa, Fla.

Location.--Lat $29^{\circ} 30^{\prime}$, long $82^{\circ} 06^{\prime}$, in sec. 27, T. $11 \mathrm{~S}$, , R. 22 E., at Cassels' dock on southeast shore of lake in town of Lochloosa.

Records ava1lable.--U. S. Geolog1cal Survey: July 1942 to December 1952, da1ly gage helghts.

Gage.--Staff gage. Datum of gage $1 \mathrm{~s} 55.83 \mathrm{ft}$ above mean sea level, datum of 1929.

Historical data.--Flood of October 1941 reached a stage of $6.2 \mathrm{ft}$.

Annual peak stages

\begin{tabular}{c|c|c||c|c|c}
\hline Calendar year & Date & $\begin{array}{c}\text { Gage he1ght } \\
\text { (feet) }\end{array}$ & Calendar year & Date & $\begin{array}{c}\text { Gage he1ght } \\
\text { feet) }\end{array}$ \\
\hline 1941 & October & 6.2 & 1947 & Nov. 14 & 5.18 \\
1943 & Jan. 1 & 2.16 & 1948 & Mar. 16 & 6.11 \\
1944 & Oct. 23-26 & 2.92 & 1949 & Oct. 10-12 & 3.84 \\
1945 & Dec. 31 & 3.66 & 1950 & Oct. 23 & 4.60 \\
1946 & Oct. 11 & 5.50 & 1951 & Jan. 13, 3. & 3.34 \\
\hline
\end{tabular}




\section{St. Johns River Basin}

(36) Lochloosa Lake Outlet near Lochloosa, Fla.

Location.--Lat $29^{\circ} 29^{\prime} 10^{\prime \prime}$, long $82^{\circ} 06^{\prime} 10^{\prime \prime}$, in sec. 3, T. 12 S., R. 22 E., on right bank at upstream side of wingwall of culvert on U. S. H1ghway 301 and State Highway $200,1.3 \mathrm{mlles}$ south of Lochloosa, and 2.4 miles north of Island Grove.

Drainage area.--Indeterminate. Total drainage area of Orange Lake Outlet and Lochloosa Lake Outlet above highway is $323 \mathrm{sq} \mathrm{ml}$.

Records avallable.--U. S. Geological Survey: January 1947 to December 1953, daily gage helghts and discharges.

Gage.--Water-stage recorder. Datum of gage is $55.41 \mathrm{ft}$ above mean sea level, datum of 1929. Prior to Mar. 28, 1947, staff gage at same site and datum.

Stage-discharge relation.--Defined by current-meter measurements below 332 cfs. Considerable shifting at all stages.

Remarks.--Orange and Lochloosa Lakes are connected by Cross Creek through wh1ch there may be a natural diversion from one lake to the other.

Annual peak stages and discharges

\begin{tabular}{|c|c|c|c|c|c|c|c|}
\hline $\begin{array}{l}\text { Calendar } \\
\text { year }\end{array}$ & Date & $\begin{array}{l}\text { Gage } \\
\text { height } \\
\text { (feet) }\end{array}$ & $\begin{array}{l}\text { Discharge } \\
\text { (cfs) }\end{array}$ & $\begin{array}{c}\text { Calendar } \\
\text { year }\end{array}$ & Date & $\begin{array}{c}\text { Gage } \\
\text { height } \\
\text { (feet) }\end{array}$ & $\begin{array}{l}\text { Discharge } \\
\text { (cfs) }\end{array}$ \\
\hline $\begin{array}{l}1947 \\
1948 \\
1949 \\
1950 \\
\end{array}$ & $\begin{array}{l}\text { Nov. } 18,19 \\
\text { Mar. } 12,13 \\
\text { Apr. } 8,9 \\
\text { Oct. } 9 \\
\text { Oct. } 22,23,24\end{array}$ & $\begin{array}{l}5.11 \\
6.04 \\
3.98 \\
- \\
4.49\end{array}$ & $\begin{array}{r}194 \\
341 \\
- \\
50 \\
138\end{array}$ & $\begin{array}{l}1951 \\
1952 \\
1953\end{array}$ & $\begin{array}{ll}\text { Dec. } & 27 \\
\text { Feb. } & 27 \\
\text { Sept. } & 30 \\
\text { Oct. } & 1-3\end{array}$ & $\begin{array}{l}3.38 \\
3.65 \\
- \\
4.45\end{array}$ & $\begin{array}{r}20 \\
45 \\
116 \\
-\end{array}$ \\
\hline
\end{tabular}

(37) Orange Creek at Orange Springs, Fla.

Location.--Lat $29^{\circ} 31^{\prime}$, long $81^{\circ} 57^{\prime}$, in sec. 25, T. 11 S., R. 23 E., on right bank at downstream side of bridge on State Highway 318 , a quarter of a mile northwest of Orange Springs, and $1 \frac{1}{4}$ miles upstream from Little Orange Creek.

Drainage area. $--431 \mathrm{sq} \mathrm{ml}$.

Records avallable.--U. S. Geological Survey: July 1942 to December 1952, daily gage helghts and per1od1c discharge measurements.

Gage.--Staff gage read once dally. Datum of gage is $19.81 \mathrm{ft}$ above mean sea level, datum of 1929. Prior to Nov. 12, 1942, staff gage at datum $3.00 \mathrm{ft}$ higher.

Stage-discharge relation.--Defined by current-meter measurements below 1,500 cfs. Relation is fairly permanent.

H1storical data.-Flood of October 1941 reached a stage of $10.6 \mathrm{ft}$, present datum, from Information by observer (discharge, 2,500 cfs, from rating curve extended above $1,490 \mathrm{cfs}$ which was the result of a discharge measurement made on Nov. 16, 1941). 
St. Johns R1ver Basin

(37) Orange Creek at Orange Springs, Fla.--Cont1nued

Annual peak stages and discharges

\begin{tabular}{c|c|c|c||c|c|c|c}
\hline $\begin{array}{c}\text { Calendar } \\
\text { year }\end{array}$ & Date & $\begin{array}{c}\text { Gage } \\
\text { height } \\
\text { (feet) }\end{array}$ & $\begin{array}{c}\text { Discharge } \\
\text { (cfs) }\end{array}$ & $\begin{array}{c}\text { Calendar } \\
\text { year }\end{array}$ & Date & $\begin{array}{c}\text { Gage } \\
\text { height } \\
\text { (feet) }\end{array}$ & $\begin{array}{c}\text { Discharge } \\
\text { (cfs) }\end{array}$ \\
\hline 1941 & October & 10.6 & 2,500 & 1947 & Sept. 23 & 8.04 & 980 \\
1943 & Sept. 19 & 7.00 & 500 & 1948 & Mar. 10 & 8.62 & 1,290 \\
1944 & Oct. 19 & 7.64 & 760 & 1949 & Apr. 5 & 7.50 & 700 \\
1945 & Dec. 29 & 7.26 & 590 & 1950 & Sept. 6 & 8.40 & 1,180 \\
1946 & oct. 9 & 8.04 & 980 & 1951 & Oct. 2 & 7.40 & 650 \\
\hline
\end{tabular}

Note.-Gage helghts for years 1943-52 are maximum observed on once da1ly bas1s; peak stage may have been slightly higher.

(38) Oklawaha River near Orange Springs, Fla.

Location.--Lat $29^{\circ} 30^{\prime} 15^{\prime \prime}$, long $81^{\circ} 54^{\prime} 45^{\prime \prime}$, In sec. 29, T. 11 S., R. 24 E., on left bank at Jordans Ferry and mouth of Orange Creek, 2 miles east of Orange Springs.

Drainage area. $--2,010 \mathrm{sq} \mathrm{ml}$, approximately (1ncluding Orange Creek).

Records ava1lable.--U. S. Geologlcal Survey: February 1930 to December 1952, da1ly gage he1ghts and discharges.

Gage.--Staff gage read tw1ce da1ly. Datum of gage 1s $7.12 \mathrm{ft}$ above mean sea level, datum of 1929 (Corps of Englneers benchmark). Prior to Oct. 1, 1936, at s1te a quarter of a m1le downstream at same datum.

Stage-discharge relation.--Defined by current-meter measurements below 8,000 cfs; considerable shifting in lower 11mits.

Annual peak stages and discharges



a At site then in use; $12.00 \mathrm{ft}$, present site, from floodmarks. 


\section{St. Johns River Basin}

(39) Oklawaha Rlver at Riverside Landing, near Orange Springs, Fla.

Location.--Lat $29^{\circ} 30^{\prime}$, long $81^{\circ} 48^{\prime}$, in sec. 33, T. Il S., R. 25 E., at Riverside Landing on right bank near boat dock, $8 \frac{1}{4} \mathrm{miles}$ east of Orange Springs.

Dra1nage area.--2,100 sq $\mathrm{ml}$, approximately.

Records avallable.--U. S. Geological Survey: October 1943 to December 1953, daily gage heights and discharges.

Gage.--Water-stage recorder. Datum of gage is at mean sea level, datum of 1929 (Corps of Engineers benchmark).

Stàge-discharge relation.--Defined by current-meter measurements below 5,800 cfs.

Annual peak stages and discharges

\begin{tabular}{c|c|c|c|c|c|c|c}
\hline $\begin{array}{c}\text { Calendar } \\
\text { year }\end{array}$ & Date & $\begin{array}{c}\text { Gage } \\
\text { height } \\
\text { (feet) }\end{array}$ & $\begin{array}{c}\text { Discharge } \\
\text { (cfs) }\end{array}$ & $\begin{array}{c}\text { Calendar } \\
\text { year }\end{array}$ & Date & $\begin{array}{c}\text { Gage } \\
\text { height } \\
\text { (feet) }\end{array}$ & $\begin{array}{c}\text { Discharge } \\
\text { (cfs) }\end{array}$ \\
\hline 1944 & Oct. 23. & 8.54 & 5,550 & 1949 & Sept. 2 & 7.74 & 4,370 \\
1945 & Sept. 20 & 8.25 & 5,040 & 1950 & Sept. 10 & 9.50 & 7,320 \\
1946 & Sept. 21 & 7.60 & 4,100 & 1951 & Oct. 3 & 7.57 & 4,180 \\
1947 & Sept. 24 & 8.57 & 5,720 & 1952 & Feb. 27 & 7.35 & 3,840 \\
1948 & Mar. 12 & 8.83 & 6,060 & 1953 & Oct. 4 & 8.30 & 5,480 \\
\hline
\end{tabular}

(40) South Fork Black Creek near Penney Farms, Fla.

Location.--Lat $29^{\circ} 59^{\prime}$, long $81^{\circ} 51^{\prime}$, in sec. 13, T. $6 \mathrm{~S} ., \mathrm{R} .24 \mathrm{E}$, , on right bank about $20 \mathrm{ft}$ downstream from bridge on State Highway 16 , half a mile downstream from Greens Creek, $2 \frac{1}{2} \mathrm{mlles}$ west of Penney Farms, and 10 miles west of Green Cove Springs.

Drainage area. $--134 \mathrm{sq} \mathrm{mi}$.

Records ava1lable.--U. S. Geological Survey: November 1939 to December 1953, daily gage helghts and discharges.

Gage.--Water-stage recorder. Datum of gage $1 \mathrm{~s} 9.82 \mathrm{ft}$ above mean sea level, datum of 1929 Tlevels by Corps of Engineers). Prior to July 18, 1940, staff gage at same site and datum.

Stage-discharge relation.--Defined by current-meter measurements below 8,000 cfs.

Annual peak stages and discharges

\begin{tabular}{|c|c|c|c|c|c|c|c|}
\hline $\begin{array}{l}\text { Calendar } \\
\text { year }\end{array}$ & Date & $\begin{array}{l}\text { Gage } \\
\text { height } \\
\text { (feet) }\end{array}$ & $\begin{array}{l}\text { Discharge } \\
\text { (cfs) }\end{array}$ & $\begin{array}{l}\text { Calendar } \\
\text { year }\end{array}$ & Date & $\begin{array}{l}\text { Gage } \\
\text { height } \\
\text { (feet) }\end{array}$ & $\begin{array}{l}\text { Discharge } \\
\text { (cfs) }\end{array}$ \\
\hline 1940 & Dec. 25 & 14.16 & & $\begin{array}{l}1947 \\
1948\end{array}$ & Sept. 24 & $\begin{array}{l}22.60 \\
19.24\end{array}$ & $\begin{array}{l}9,390 \\
5,970\end{array}$ \\
\hline $\begin{array}{l}1941 \\
1942 \\
1943\end{array}$ & $\begin{array}{ll}\text { Oct. } & 21,22 \\
\text { Mar. } & 15 \\
\text { Aug. } & 15\end{array}$ & $\begin{array}{l}21.65 \\
14.74 \\
19.87\end{array}$ & $\begin{array}{l}8,560 \\
1,850 \\
6,240\end{array}$ & $\begin{array}{l}1949 \\
1950\end{array}$ & $\begin{array}{ll}\text { Oct. } & 2 \\
\text { Oct. } & 19\end{array}$ & $\begin{array}{l}14.77 \\
21.25\end{array}$ & $\begin{array}{l}1,900 \\
7,970\end{array}$ \\
\hline $\begin{array}{l}1944 \\
1945\end{array}$ & $\begin{array}{l}\text { Oct. } 19 \\
\text { Sept. } 17\end{array}$ & $\begin{array}{l}26.33 \\
17.13\end{array}$ & $\begin{array}{r}13,900 \\
3,940\end{array}$ & $\begin{array}{l}1951 \\
1952 \\
1953\end{array}$ & $\begin{array}{ll}\text { Nov. } & 17 \\
\text { Oct. } & 22 \\
\text { Apr. } & 20\end{array}$ & $\begin{array}{l}10.95 \\
13.71 \\
19.40\end{array}$ & $\begin{array}{r}970 \\
1,470 \\
5,790\end{array}$ \\
\hline 1946 & May & 13.91 & 1,530 & & & & \\
\hline
\end{tabular}




\section{St. Johns R1ver Basin}

(41) North Fork Black Creek near M1ddleburg, Fla.

Location.--Lat $30^{\circ} 06^{\prime} 50^{\prime \prime}$, long $81^{\circ} 54^{\prime} 35^{\prime \prime}$, in sec. 33, T. 4 S., R. 24 E., at left bank, a third or a m1le upstream frc $=$ B1g Branch, 4 miles northwest of Middleburg, and $6 \frac{3}{4}$ m1les upstream from confluence with South Fork.

Drainage area. $--174 \mathrm{sq} \mathrm{mi}$.

Records ava1lable.--U. S. Geological Survey: November 1931 to December 1953, da1ly gage helghts and discharges.

Gage.--Staff gage reaj twice dally. Datum of gage is $0.62 \mathrm{ft}$ above mean sea level, datum of 1929 (levels by Corps of Engineers). Prior to Mar. 29, 1933, at site three-e1ghths of a m1le downstream at different datum.

Stage-discharge relation.--Defined by current-meter measurements below 7,000 $\mathrm{cfs}$.

H1storical data.--Maximum stage known, $25.3 \mathrm{ft}$ in June 1919, from information by old res1dent (discharge, i5, $000 \mathrm{cfs}$, from rating curve extended above 7,000 cfs).

Annual peak stages and discharges

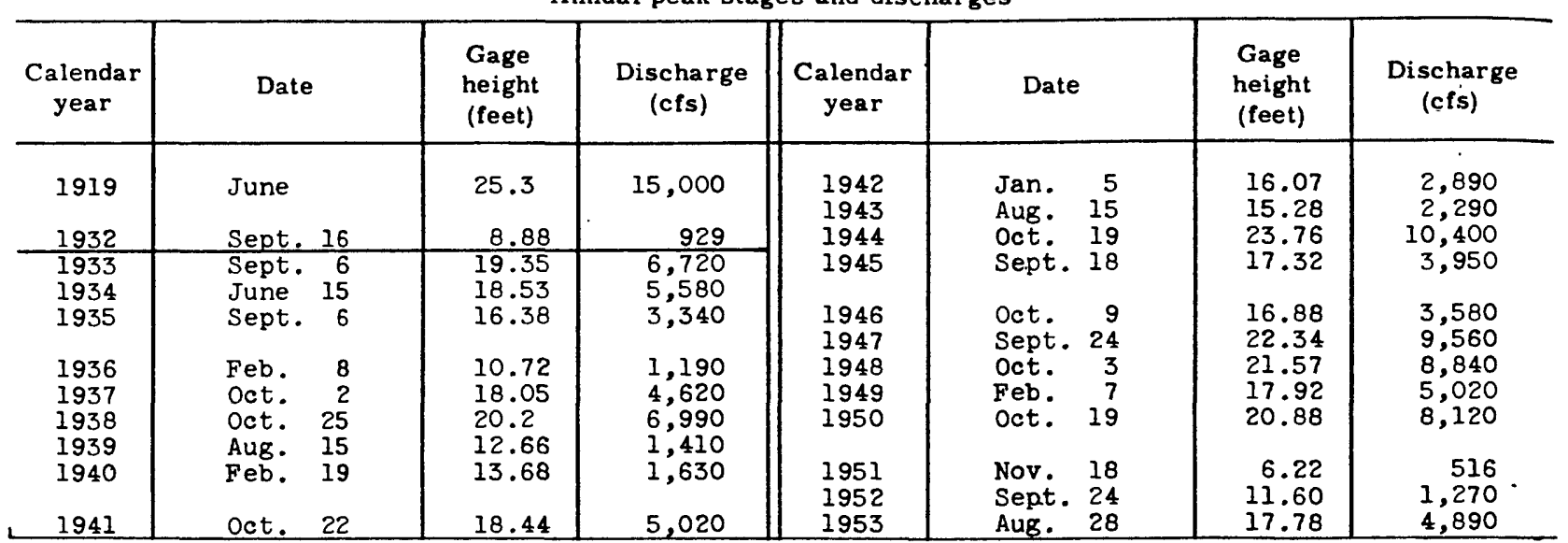

Moultrie Creek Basin

(42) Moultrle Creek near St. August1ne, Fla.


downstream from bridge on K1ngs Road, two-fifths of a mile upstream from Fort Peyton Branch, and 5 miles southwest of St. Augustine.

Drainage area. $--23.3 \mathrm{sq} \mathrm{m} 1$.

Records ava1lable.--U. S. Geological Survey: November 1939 to December 1953, da1ly gage heights and discharges.

Gage.--Water-stage recorder and wooden control. Datum of gage is $2.17 \mathrm{ft}$ above mean sea level, datum of 1929 .

Stage-d1scharge relation.--Defined by current-meter measurements below $1,100 \mathrm{cfs}$.

Historical data.--Maximum stage known, about $13 \mathrm{ft}$ in 1919, from information by local resident. 


\section{Moultrie Creek Basin}

(42) Moultrie Creek near St. Augustine, Fla.--Continued

Annual peak stages and discharges

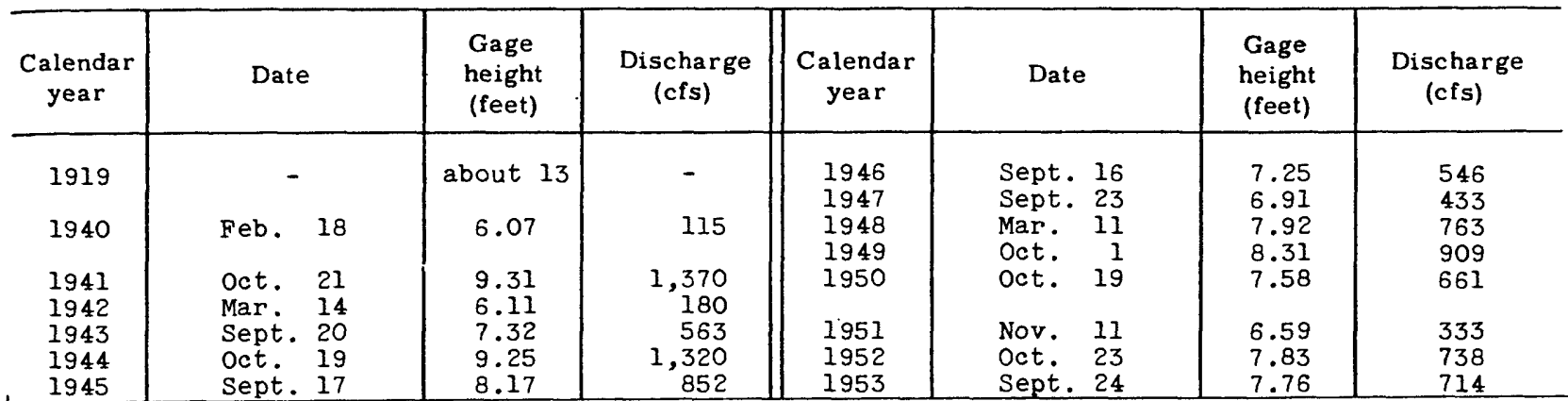

Lake Okeechobee and the Everglades

(43) Lake Okeechobee, Fla.

Location.--Center of lake, lat $26^{\circ} 57^{\prime}$, long $80^{\circ} 50^{\prime}$, in southern Florida.

Records ava1lable.--Everglades Dra1nage District: 1912 to 1931, intermittent records of stage at various locations on the lake. Rellability of these records is questionable due to uncertainty of gage datum and location with respect to lake outlets and consequent drawdown and lockage effects. U. S. Geological Survey: Apr1l 1931 to December 1953, da1ly gage heights collected and furnished by Corps of Engineers.

Gage.--Three staff gages, at Hurricane Gate No. 2, Hurricane Gate No. 6, and Port Mayaca, read once da1ly. Oct. 1, 1941, to Dec. 31, 1950, seven staff gages at varlous locations on rim of lake. Prior to oct. 1, 1941, staff gage at st. Lucie Canal.

Datum of gages is at mean sea level (levels by Corps of Engineers). Oct. 1, 1933, to June 30, 1947, datum of gage or gages was $1.44 \mathrm{ft}$ below mean sea level (levels by Corps of Engineers). Prior to 0ct. 1, 1933, datum of gage was $1.01 \mathrm{ft}$ below mean sea level. (All gage heights tabulated below have been converted to present datum, at mean sea level).

Remarks.--Stage of lake regulated by gates at several lake outlets. Since Oct. 1, 1940, average elevation is computed from readings of 3 or more gages.

\begin{tabular}{|c|c|c|c|c|c|}
\hline Calendar year & Date & $\begin{array}{c}\text { Tlevation, } \\
\text { feet above } \\
\text { mean sea level }\end{array}$ & Calendar year & Date & $\begin{array}{c}\text { Elevation, } \\
\text { feet above } \\
\text { mean sea level }\end{array}$ \\
\hline $\begin{array}{l}1930 \\
1931 \\
1932 \\
1933 \\
1934 \\
1935 \\
1936 \\
1937 \\
1938 \\
1939 \\
1940 \\
1941\end{array}$ & $\begin{array}{ll}\text { July } & 20-23 \\
\text { Jan. } & 6 \\
\text { Nov. } & 12,27 \\
\text { Sept. } & 4 \\
\text { Oct. } & 8,9,12 \\
\text { Jan. } & 23 \\
& \\
\text { July } & 20 \\
\text { Dec. } & 2 \\
\text { Jan. } & 4 \\
\text { Nov. } & 1 \\
\text { Feb. } 22 \\
\text { Oct. } 23,25\end{array}$ & $\begin{array}{l}17.5 \\
16.4 \\
13.6 \\
20.1 \\
15.4 \\
15.3 \\
15.4 \\
15.8 \\
15.1 \\
15.90 \\
15.78 \\
15.21\end{array}$ & $\begin{array}{l}1942 \\
1943 \\
1944 \\
1945 \\
1946 \\
1947 \\
1948 \\
1949 \\
1950 \\
1951 \\
1952 \\
1953\end{array}$ & $\begin{array}{l}\text { Feb. } 25 \\
\text { Dec. } 16,17 \\
\text { Jan. } 1 \\
\text { Oct. } 31 \\
\text { Mar. } 19 \\
\text { Nov. } 2 \\
\text { Oct. } 18,19 \\
\text { Jan. } 1 \\
\text { Jan. } 2 \\
\text { Oct. } 13 \\
\text { Oct. } 28,29 \\
\text { Oct. } 29\end{array}$ & $\begin{array}{l}15.20 \\
13.92 \\
13.75 \\
15.78 \\
15.46 \\
18.77 \\
17.77 \\
15.53 \\
15.16 \\
15.97 \\
15.81 \\
17.66\end{array}$ \\
\hline
\end{tabular}

Note.--Maximum stages shown above computed from readings of one gage prior to oct. 1 , 1941 , and from average of 3 or more gages at various locations subsequent to that date. 
(44) Fisheat1ng Creek at Palmdale, Fla.

Location.--Lat $26^{\circ} 56^{\prime}$, long $81^{\circ} 19^{\prime}$, in sec. 3, T. 41 S., R. 30 E., near right bank on downstream slde of bridge on U. S. Highway 27,1 mile south of Palmdale, and 16 miles upstream from Lake Okeechobee.

Drainage area. $--435 \mathrm{sq} \mathrm{m1}$, approximately.

Records avallable.--U. S. Geological Survey: Apr1l 1931 to December 1953, da1ly gage he1ghts and discharges.

Gage.-Water-stage recorder. Datum of gage 1s $27.19 \mathrm{ft}$ above mean sea level, datum of 1929. Prior to Mar. 16, 1949, staff gage at same s1te and datum.

Stage-d1scharge relat1on.--Def1ned by current-meter measurements below $21,000 \mathrm{cf}$. Cons1derable shifting at all stages.

Annual peak stages and discharges

\begin{tabular}{|c|c|c|c|c|c|c|c|}
\hline $\begin{array}{c}\text { Calendar } \\
\text { year }\end{array}$ & Date & $\begin{array}{c}\text { Gage } \\
\text { height } \\
\text { (feet) }\end{array}$ & $\begin{array}{l}\text { Discharge } \\
\text { (cfs) }\end{array}$ & $\begin{array}{c}\text { Calendar } \\
\text { year }\end{array}$ & Date & $\begin{array}{c}\text { Gage } \\
\text { height } \\
\text { (feet) }\end{array}$ & $\begin{array}{c}\text { Discharge } \\
\text { (cfs) }\end{array}$ \\
\hline $\begin{array}{l}1932 \\
1933 \\
1934 \\
1935\end{array}$ & $\begin{array}{lr}\text { Sept. } & 13 \\
\text { Sept. } & 6 \\
\text { Aug. } & 9 \\
\text { Sept. } & 7\end{array}$ & $\begin{array}{c}8.26 \\
8.60 \\
- \\
6.42\end{array}$ & $\begin{array}{r}5,570 \\
6,460 \\
920 \\
1,480\end{array}$ & $\begin{array}{l}1943 \\
1944 \\
1945\end{array}$ & $\begin{array}{l}\text { Oct. } 5 \\
\text { Sept. } 2 \\
\text { Sept. } 17\end{array}$ & $\begin{array}{l}7.30 \\
6.30 \\
9.18\end{array}$ & $\begin{array}{l}3,620 \\
1,500 \\
8,980\end{array}$ \\
\hline $\begin{array}{l}1936 \\
1937 \\
1938 \\
1939 \\
1940\end{array}$ & $\begin{array}{l}\text { June } 15,16 \\
\text { July } 1 \\
\text { Oct. } 17 \\
\text { Aug. } 29 \\
\text { Sept. } 12\end{array}$ & $\begin{array}{l}8.10 \\
6.98 \\
7.14 \\
6.92 \\
6.92\end{array}$ & $\begin{array}{l}5,800 \\
3,010 \\
3,230 \\
2,790 \\
3,090\end{array}$ & $\begin{array}{l}1946 \\
1947 \\
1948 \\
1949 \\
1950\end{array}$ & $\begin{array}{l}\text { Sept. } 23 \\
\text { Sept. } 19 \\
\text { Sept. } 24 \\
\text { Aug. } 29 \\
\text { Sept. } 9\end{array}$ & $\begin{array}{r}5.96 \\
11.06 \\
10.52 \\
7.86 \\
5.88\end{array}$ & $\begin{array}{r}946 \\
16,400 \\
14,500 \\
5,300 \\
583\end{array}$ \\
\hline $\begin{array}{l}1941 \\
1942\end{array}$ & $\begin{array}{ll}\text { Apr. } & 10 \\
\text { Feb. } & 26\end{array}$ & $\begin{array}{l}6.70 \\
7.04\end{array}$ & $\begin{array}{l}2,790 \\
3,260\end{array}$ & $\begin{array}{l}1951 \\
1952 \\
1953\end{array}$ & $\begin{array}{lr}\text { Oct. } & 3 \\
\text { Oct. } & 21 \\
\text { Oct. } & 10\end{array}$ & $\begin{array}{r}12.44 \\
7.77 \\
8.53\end{array}$ & $\begin{array}{r}31,400 \\
4,920 \\
7,520\end{array}$ \\
\hline
\end{tabular}

(45) Indian Pra1rie Canal near Okeechobee, Fla.

Location.--Lat $27^{\circ} 04^{\prime}$, long $80^{\circ} 59^{\prime}$, in sec. 24, T. 39 S., R. 33 E., on right bank at downstream slde of bridge on State H1ghway $78,2 \mathrm{miles}$ upstream from shore of Lake Okeechobee corresponding to lake elevation of $12.5 \mathrm{ft}$ above mean sea level, and $15 \mathrm{mlles}$ southwest of town of Okeechobee.

Records ava1lable.--U. S. Geological Survey: Apr1l 1931 to February 1933, da1ly gage helghts and discharges, at site 3 mlles upstream publ1shed as "near Lakeport". June 1939 to December 1950, da1ly gage helghts and discharges. Corps of Eng1neers: January 1951 to December 1953, da1ly gage helghts and discharges.

Gage.--Water-stage recorder. Datum of gage is at mean sea level, unadjusted (levels by Corps of Eng1neers). Apr1l 1931 to February 1933, at s1te 3 miles upstream at different datum.

Stage-discharge relation.--Poorly defined by current-meter measurements, affected by backwater from Lake Okeechobee and hyacinth fams. 
Lake Okeechobee and the Everglades

(45) Indian Prairle Canal near Okeechobee, Fla.--Continued

Annual peak stages and discharges

\begin{tabular}{|c|c|c|c|c|c|c|c|}
\hline $\begin{array}{l}\text { Calendar } \\
\text { year }\end{array}$ & Date & $\begin{array}{l}\text { Gage } \\
\text { height } \\
\text { (feet) }\end{array}$ & $\begin{array}{c}\text { Discharge } \\
\text { (cfs) }\end{array}$ & $\begin{array}{c}\text { Calendar } \\
\text { year }\end{array}$ & Date & $\begin{array}{l}\text { Gage } \\
\text { height } \\
\text { (feet) }\end{array}$ & $\begin{array}{l}\text { Discharge } \\
\quad(c f s)\end{array}$ \\
\hline $\begin{array}{l}1931 \\
1932\end{array}$ & $\begin{array}{lr}\text { Mar. } & 7 \\
\text { Sept. } & 15\end{array}$ & $\begin{array}{l}20.4 \\
21.00\end{array}$ & $\begin{array}{r}a 900 \\
1,140\end{array}$ & \multirow{3}{*}{$\begin{array}{l}1947 \\
1948 \\
1949 \\
1950 \\
1951 \\
1952 \\
1953\end{array}$} & \multirow{2}{*}{$\begin{array}{lr}\text { Sept. } & 18 \\
\text { oct. } & 12 \\
\text { oct. } & 5 \\
\text { Oct. } & 16 \\
\text { Aug. } & 28 \\
\text { Oct. } & 21\end{array}$} & \multirow{2}{*}{$\begin{array}{c}- \\
19.10 \\
- \\
18.50 \\
18.44 \\
14.05\end{array}$} & \multirow{2}{*}{$\begin{array}{c}1,610 \\
\overline{1,880} \\
- \\
1.880 \\
b_{149}^{8}\end{array}$} \\
\hline $\begin{array}{l}1939 \\
1940\end{array}$ & $\begin{array}{l}\text { Aug. } 12 \\
\text { Sept. } 11,12\end{array}$ & $\begin{array}{l}17.62 \\
17.46\end{array}$ & $\begin{array}{r}704 \\
\text { b } 517\end{array}$ & & & & \\
\hline $\begin{array}{l}1941 \\
1942 \\
1943 \\
1944 \\
1945 \\
1946\end{array}$ & $\begin{array}{ll}\text { July } & 18 \\
\text { June } & 14 \\
\text { Aug. } & 31 \\
\text { Sept. } & 12 \\
\text { Sept. } & 16 \\
& \\
\text { Aug } & 4\end{array}$ & $\begin{array}{l}17.22 \\
17.49 \\
17.37 \\
15.73 \\
18.05 \\
16.34\end{array}$ & $\begin{array}{r}1,090 \\
1,240 \\
728 \\
77 \\
1,540 \\
\\
315\end{array}$ & & $\begin{array}{lr}\text { Oct. } & 3 \\
\text { Oct. } & 9 \\
\text { Oct. } & 20 \\
\text { Oct. } & 21 \\
\text { Oct. } & 27\end{array}$ & $\begin{array}{c}18.16 \\
- \\
- \\
18.01 \\
18.76\end{array}$ & $\begin{array}{c}- \\
b 1,500 \\
b 1,500 \\
- \\
b_{2}, 480\end{array}$ \\
\hline
\end{tabular}

Estimated.

b Maximum daily.

(46) Alligator Lake near Ashton, Fla.

Location.--Lat $28^{\circ} 13^{\prime} 59^{\prime \prime}$, long $81^{\circ} 11^{\prime} 25^{\prime \prime}$, In sec. 14, T. 26 S., R. 31 E., on dock on northeast shore of lake, $300 \mathrm{ft}$ east of canal connecting Alilgator Lake and Lake L1zzle, $350 \mathrm{ft}$ southwest of U. S. Highway 192, and 4 m1les east of Ashton.

Records avallable.--U. S. Geologlcal Survey: November 1941 to December 1953, da1ly gage helghts. Gage.--Staff gage read once da1ly. Datum of gage is $60.74 \mathrm{ft}$ above mean sea level (levels by Corps of Engineers).

H1stor1cal data.--H1gh water of June 1934, reached a stage of about $7.0 \mathrm{ft}$, from information by observer.

Annual peak stages

\begin{tabular}{|c|c|c|c|c|c|}
\hline Calendar year & Date & $\begin{array}{c}\text { Gage helght } \\
\text { (feet) }\end{array}$ & Calendar year & . Date & $\begin{array}{l}\text { Gage he 1ght } \\
\text { (feet) }\end{array}$ \\
\hline $\begin{array}{l}1934 \\
1942 \\
1943 \\
1944 \\
1945 \\
1946 \\
1947\end{array}$ & $\begin{array}{ll}\text { June } & - \\
\text { July } & 6 \\
\text { Sept. } & 22,23 \\
\text { Oct. } 21 & \\
\text { Sept. } & 19,20 \\
& \\
\text { Oct. } 10 & \\
\text { Oct. } 13 & \end{array}$ & $\begin{array}{l}7.0 \\
4.96 \\
4.55 \\
5.64 \\
5.28 \\
3.92 \\
5.28\end{array}$ & $\begin{array}{l}1948 \\
1949 \\
1950 \\
1951 \\
1952 \\
1953\end{array}$ & $\begin{array}{l}\text { Oct. } 6,7 \\
\text { Oct. } 6 \\
\text { Jan. } 1 \\
\text { Nov. } 23 \\
\text { Oct. } 28-31 ; \\
\text { Nov. } 1,3 \\
\text { Oct. } 11\end{array}$ & $\begin{array}{l}5.48 \\
4.80 \\
3.50 \\
4.32 \\
4.00 \\
5.54\end{array}$ \\
\hline
\end{tabular}




\section{Lake Okeechobee and the Everglades}

(47) Hart Lake near Narcoossee, Fla.

Location.--Lat $28^{\circ} 22^{\prime} 40^{\prime \prime}$, long $81^{\circ} 13^{\prime} 30^{\prime \prime}$, in sec. 21 , T. 24 S., R. 31 E., near west shore of lake, 1.2 miles east of State Highway 15 , and 5.5 miles north-northeast of Narcoossee.

Records ava1lable.--U. S. Geological Survey: November 1941 to December 1953, da1ly gage helghts. Gage.--Staff gage read once dally. Datum of gage is $55.74 \mathrm{ft}$ above mean sea level (levels by Corps of Engineers). Prior to Dec. 11, 1942, staff gage at same site and at datum $2.22 \mathrm{rt}$ higher.

\begin{tabular}{|c|c|c|c|c|c|}
\hline Calendar year & Date & $\begin{array}{c}\text { Gage height } \\
\text { (feet) }\end{array}$ & Calendar year & Date & $\begin{array}{c}\text { Gage helght } \\
\text { (feet) }\end{array}$ \\
\hline $\begin{array}{l}1942 \\
1943 \\
1944 \\
1945 \\
1946 \\
1947 \\
\end{array}$ & $\begin{array}{ll}\text { July } & 18 \\
\text { Sept. } & 23 \\
\text { Oct. } 27-30 \\
\text { Sept. } 20 \\
\text { Oct. } 1,2 \\
\text { Oct. } 3,2\end{array}$ & $\begin{array}{r}5.65 \\
6.41 \\
5.78 \\
9.13 \\
5.13 \\
7.82 \\
\end{array}$ & $\begin{array}{l}1948 \\
1949 \\
1950 \\
1951 \\
1952 \\
1955\end{array}$ & $\begin{array}{l}\text { Oct. } 7 \\
\text { Oct. } 6,7 \\
\text { Oct. } 24 \\
\text { Oct. } 7-9 \\
\text { Oct. } 30,31 \\
\text { Oct. } 2,4\end{array}$ & $\begin{array}{l}8.83 \\
6.98 \\
5.76 \\
5.24 \\
5.42 \\
8.30\end{array}$ \\
\hline
\end{tabular}

a Present datum.

(48) East Tohopekal1ga Lake at S:. Cloud, Fla.

Location.--Lat $28^{\circ} 15^{\prime} 29^{\prime \prime}$, long $81^{\circ} 16^{\prime} 57^{\prime \prime}$, In sec. 2, T. 2: S., R. 30 E., in boat basin on south side of lake at St. Cloud.

Records ava1lable.--U. S. Geological Survey: November 10tI to December 1953, da1ly gage he1ghts.

Gage.--Staff gage read once dally. Datum of gage is 51 . II ft above mean sea level (Corps of Englneers benchmark). Prlor to Mar. 5, 1943, staff gkse was located on former city pler at approximately same location, at datum $2.61 \mathrm{ft}$ higher.

Historical data.-Flood of July 1930 reached a stage of $1: .79 \mathrm{ft}$, present datum, from information by Mr. J. C. Gallatin, St. Cloud.

\begin{tabular}{|c|c|c|c|c|c|}
\hline Calendar year & Date & $\begin{array}{l}\text { Gage helght } \\
\text { feet) }\end{array}$ & Calende- year & Date & Gage helght \\
\hline $\begin{array}{l}1930 \\
1942 \\
1943 \\
1944 \\
1945 \\
1946\end{array}$ & $\begin{array}{ll}\text { July } & - \\
\text { Mar. } & 31 \\
\text { Oct. } 10,11 & \\
\text { Nov. } 3,4,6 \\
\text { Sept. } 24,25 \\
\text { Jan. } \quad 1\end{array}$ & $\begin{array}{r}10.79 \\
{ }^{\circ} 6.69 \\
6.66 \\
6.70 \\
10.37 \\
6.38\end{array}$ & $\begin{array}{l}195 \\
195 \\
195 \\
195 \\
195= \\
19=5 \\
19=5\end{array}$ & $\begin{array}{l}\text { Oct. } 13 \\
\text { Oct. } 13,14 \\
\text { Oct. } 7-14 \\
\text { Jan. } 1,2 \\
\text { Nov. } 29 \\
\text { Jan. } 1 \\
\text { Oct. } 12,13\end{array}$ & $\begin{array}{r}9.37 \\
10.06 \\
8.67 \\
6.66 \\
7.28 \\
6.68 \\
10.63\end{array}$ \\
\hline
\end{tabular}


Lake Okeechobee and the Everglades

(49) Lake Tohopekaliga at K1ssimmee, Fla.

Locatión.--Lat $28^{\circ} 17^{\prime} 20^{\prime \prime}$, long $81^{\circ} 24^{\prime} 22^{\prime \prime}$, In sec. 27, T. 25 S., R. 29 E., on southeast end of munlclpal dock on north shore of lake at K1ssimmee.

Records ava1lable.--U. S. Geolog1cal Survey: January 1942 to December 1953, da1ly gage heights.

Gage.--Water-stage recorder. Datum of gage 1s at mean sea level, datum of 1929. Prior to June 15, 1950, water stage recorder was located on the west shore of lake about 9 miles south of K1ssimmee. Datum of gage at this location was $50.36 \mathrm{ft}$ above mean sea level (levels by Corps of Engineers). (All gage helghts tabulated below have been reduced to present datum, at mean sea level).

Annual peak stages

\begin{tabular}{|c|c|c|c|c|c|}
\hline Calendar year & Date & $\begin{array}{l}\text { Elevation, } \\
\text { feet above } \\
\text { mean sea level }\end{array}$ & Calendar year & Date & $\begin{array}{l}\text { Elevation, } \\
\text { feet above } \\
\text { mean sea level }\end{array}$ \\
\hline \multirow{2}{*}{$\begin{array}{l}1942 \\
1943 \\
1944 \\
1945\end{array}$} & \multirow{2}{*}{$\begin{array}{l}\text { July } 8 \\
\text { Oct. } 7,11,12 \\
\text { Sept. } 24,25 \\
\text { Sept. } 23,24 \\
\text { Oct. } 11 \\
\text { Oct. } 13\end{array}$} & \multirow{2}{*}{$\begin{array}{l}55.32 \\
54.56 \\
55.12 \\
58.32 \\
55.62 \\
58.5\end{array}$} & $\begin{array}{l}1948 \\
1949 \\
1950 \\
\end{array}$ & $\begin{array}{l}\text { Oct. } 13 \\
\text { Oct. } \overline{-} \\
\text { Jan. } 2-5 \\
\end{array}$ & $\begin{array}{l}57.79 \\
57.62 \\
55.75 \\
\end{array}$ \\
\hline & & & $\begin{array}{l}1951 \\
1952 \\
1953\end{array}$ & $\begin{array}{lr}\text { Nov. } & 26 \\
\text { Jan. } & 1 \\
\text { Oct. } 10, & 11\end{array}$ & $\begin{array}{l}55.70 \\
55.02 \\
58.62\end{array}$ \\
\hline
\end{tabular}

(50) Cypress Lake near St. Cloud, Fla.

Location.--Lat $28^{\circ} 03^{\prime} 40^{\prime \prime}$, long $81^{\circ} 19^{\prime} 58^{\prime \prime}$, in SW $\frac{1}{4}$ sec. 8, T. 28 S., R. 30 E., about $580 \mathrm{ft}$ from south shore of lake, $1,600 \mathrm{ft}$ east of head of Cypress-Hatchineha Canal, and about $13 \mathrm{mlles}$ south of St. Cloud.

Records available.--U. S. Geological Survey: January 1942 to December 1953, daily gage he1ghts.

Gage.-Water-stage recorder. Datum of gage is $48.78 \mathrm{ft}$ above mean sea level, datum of 1929 (ievels by Corps of Englneers). Prior to June 6, 1950, at site on northwest shore of lake at same datum.

Annual peak stages

\begin{tabular}{|c|c|c|c|c|c|}
\hline Calendar year & Date & $\begin{array}{l}\text { Gage height } \\
\text { feet) }\end{array}$ & Calendar year & Date & $\begin{array}{c}\text { Gage helght } \\
\text { (feet) }\end{array}$ \\
\hline $\begin{array}{l}1942 \\
1943 \\
1944 \\
1945 \\
1946 \\
1947 \\
\end{array}$ & $\begin{array}{l}\text { July } 7,8 \\
\text { Oct. } 10^{2} \\
\text { Nov. } 1 \\
\text { Sept. } 23,24 \\
\text { Oct. } 9-11 \\
\text { Oct. } 11\end{array}$ & $\begin{array}{l}5.99 \\
4.90 \\
5.78 \\
7.70 \\
5.40 \\
8.33 \\
\end{array}$ & $\begin{array}{l}1948 \\
1949 \\
1950 \\
1951 \\
1952 \\
1953 \\
\end{array}$ & $\begin{array}{l}\text { Oct. } 8 \\
\text { Oct. } 6,7 \\
\text { Jan. } 5,6 \\
\text { Nov. } 28,29 \\
\text { Oct. } 29 \\
\text { Oct. } 12,13\end{array}$ & $\begin{array}{l}7.54 \\
7.07 \\
5.39 \\
5.28 \\
4.87 \\
8.38\end{array}$ \\
\hline
\end{tabular}

Note.--Momentary peaks are wind-affected and elevations shown are maximum mean da1lies. 
Lake Okeechobee and the Everglades

(51) Lake Butler at Windermere, Fla.

Location.--Lat $28^{\circ} 30^{\prime}$, long $81^{\circ} 32^{\prime}$, in sec. 17, T. $23 \mathrm{~S} ., \mathrm{R} .28 \mathrm{E}$. , on east shore of lake at boat house of George A. Morley, near intersection of 9 th Avenue and Main Street, Windermere.

Records avallable.--Samuel Morley: January 1933 to November 194l, once weekly gage heights. U. S. Geological Survey: November 1941 to December 1953, daily gage heights.

Gage.--Staff gage read once daily. Datum of gage is 96.40 ft above mean sea level, datum of 1929. January 1933 to November 1941 , readings from reference point were made once weekly by Samuel Morley. Elevation of reference point was $100.17 \mathrm{ft}$ above mean sea level: (All gage helghts shown below have been reduced to elevations above mean sea level).

Historical data.--High water of September 1926 reached a stage of 101.30 ft, from information by a local resident.

\begin{tabular}{|c|c|c|c|c|c|}
\hline Calendar year & Date & $\begin{array}{c}\text { Elevation, } \\
\text { feet above } \\
\text { mean sea level }\end{array}$ & Calendar year & Date & $\begin{array}{c}\text { Elevation, } \\
\text { feet above } \\
\text { mean sea level }\end{array}$ \\
\hline $\begin{array}{l}1926 \\
1933 \\
1934 \\
1935 \\
1936 \\
1937 \\
1938 \\
1939 \\
1940 \\
1941 \\
1942\end{array}$ & $\begin{array}{ll}\text { Sept. } & - \\
\text { Sept. } & 10 \\
\text { Aug. } & 12 \\
\text { Sept. } & 29 \\
& \\
\text { Mar. } & 15 \\
\text { Oct. } & 24 \\
\text { Jan. } & 2,9 \\
\text { Oct. } & 22 \\
\text { Sept. } & 15 \\
& \\
\text { Aug. } \quad 4 \\
\text { July } \quad 11\end{array}$ &  & $\begin{array}{l}1943 \\
1944 \\
1945 \\
1946 \\
1947 \\
1948 \\
1949 \\
1950 \\
1951 \\
1952 \\
1953\end{array}$ & $\begin{array}{l}\text { Sept. } 22,23 \\
\text { Oct. } 20,21 \\
\text { Sept. } 17 \\
\text { Sept. } 24,27 \\
\text { Sept. } 24 \\
\text { Oct. } 5 \\
\text { Jan. } 1,2 \\
\text { Oct. } 24 \\
\text { Feb. } \quad 7,8 \\
\text { Mar. } 27-30 \\
\text { Oct. } \quad 1\end{array}$ & $\begin{array}{r}100.25 \\
101.06 \\
101.28 \\
100.85 \\
101.17 \\
100.98 \\
100.09 \\
99.80 \\
99.79 \\
99.86 \\
101.03\end{array}$ \\
\hline
\end{tabular}

a Maximum observed weekly elevation; peak stage may have been slightly higher.

(52) Cypress Creek at Vineland, Fla.

Location.--Lat $28^{\circ} 23^{\prime} 25^{\prime \prime}$, long $81^{\circ} 31^{\prime} 11^{\prime \prime}$, in sec. 21, T. 24 S., R, 28 E., on left bank at downstream side of bridge on State Highway 535, 1 mile west of Vineland.

Drainage area. $--31.0 \mathrm{sq} \mathrm{m} 1$.

Reccrds ava1lable.--U. S. Geologlcal Survey: August 1945 to December 1953, da1ly gage helghts $\therefore$ nd discharges.

Gage.--Water-stage recorder. Datum of gage $1896.20 \mathrm{ft}$ above mean sea level. Prior to June 13 , 1946, staff gage at same site and datum.

Stage-discharge relation.--Defined by current-meter measurements below 140 cfs. Considerable shifting at all stages. 
Lake Okeechobee and the Everglades

(52) Cypress Creek at Vineland, Fla.--Continued

Annual peak stages and discharges

\begin{tabular}{|c|c|c|c|c|c|c|c|}
\hline $\begin{array}{c}\text { Calendar } \\
\text { year }\end{array}$ & Date & $\begin{array}{c}\text { Gage } \\
\text { height } \\
\text { (feet) }\end{array}$ & $\begin{array}{c}\text { Discharge } \\
\text { (cfs) }\end{array}$ & $\begin{array}{c}\text { Calendar } \\
\text { year }\end{array}$ & Date & $\begin{array}{c}\text { Gage } \\
\text { height } \\
\text { (feet) }\end{array}$ & $\begin{array}{l}\text { Discharge } \\
\text { (cfs) }\end{array}$ \\
\hline 1945 & Sept. 16 & 3.83 & 181 & 1950 & oct. 19 & 2.87 & 36 \\
\hline $\begin{array}{l}1946 \\
1947 \\
1948 \\
1949 \\
\end{array}$ & $\begin{array}{ll}\text { Aug. } & 14 \\
\text { Sept. } & 18 \\
\text { Jan. } & 24 \\
\text { Sept. } & 29\end{array}$ & $\begin{array}{l}3.27 \\
3.51 \\
3.25 \\
3.20\end{array}$ & $\begin{array}{r}68 \\
112 \\
69 \\
64\end{array}$ & $\begin{array}{l}1951 \\
1952 \\
1953\end{array}$ & $\begin{array}{lr}\text { Aug. } & 6 \\
\text { Mar. } & 27 \\
\text { Sept. } & 27\end{array}$ & $\begin{array}{l}3.03 \\
2.53 \\
3.61\end{array}$ & $\begin{array}{r}57 \\
16 \\
135\end{array}$ \\
\hline
\end{tabular}

(53) Reedy Creek near Loughman, Fla.

Location.--Lat $28^{\circ} 15^{\prime} 48^{\prime \prime}$, long $81^{\circ} 32^{\prime} 12^{\prime \prime}$, In sec. 32 , T. $25 \mathrm{~S} ., \mathrm{R} .28 \mathrm{E}$, on left bank, $20 \mathrm{ft}$ upstream from bridge on U. S. Highways 17 and $92,2 \frac{1}{2}$ miles northeast of Loughman, and $3 \mathrm{mlles}$ downstream from Davenport Creek.

Drainage area.--117 $\mathrm{sq} \mathrm{m} 1$ (1ncludes part of watershed in Reedy Creek Swamp which is indeterminate).

Records avallable.--U. S. Geological Survey: November 1939 to December 1953, dally gage helghts and discharges.

Gage.--Water-stage recorder. Datum of gage is $64.49 \mathrm{ft}$ above mean sea level, datum of 1929 . Prior to Aug. 20, 1940, staff gage at same site and datum.

Stage-discharge relation.--Defined by current-meter measurements below 500 cfs. Some shifting and backwater from hyacinths at all stages.

Remarks.--Records do not Include diversions above station into Shingle Creek.

Annual peak stages and discharges

\begin{tabular}{c|c|c|c||c|c|c|c}
\hline $\begin{array}{c}\text { Calendar } \\
\text { year }\end{array}$ & Date & $\begin{array}{c}\text { Gage } \\
\text { height } \\
\text { (feet) }\end{array}$ & $\begin{array}{c}\text { Discharge } \\
\text { (cfs) }\end{array}$ & $\begin{array}{c}\text { Calendar } \\
\text { year }\end{array}$ & Date & $\begin{array}{c}\text { Gage } \\
\text { height } \\
\text { (feet) }\end{array}$ & $\begin{array}{c}\text { Discharge } \\
\text { (cfs) }\end{array}$ \\
\hline 1940 & July 1I & 3.14 & 215 & 1947 & Sept. 20, 21 & 4.02 & 530 \\
1941 & JuIy 26 & 3.97 & 501 & 1948 & Sept. 24 & 3.89 & 484 \\
1942 & June 13, 14 & 3.27 & - & 1949 & Aug. 30 & 3.97 & 508 \\
1943 & Sept. 8, 9 & - & 282 & 1950 & Oct. 21 & 3.71 & 381 \\
1944 & July 21, 22 & 3.34 & 304 & 1951 & Nov. 21 & 3.72 & 405 \\
1945 & Oct. 22, 23 & 4.02 & 530 & 1952 & Mar. 30, 31 & 3.22 & 207 \\
1946 & Sept. 19 & 3.92 & 487 & 1953 & Aug. 30 & 4.08 & 513 \\
\hline
\end{tabular}


Lake Okeechobee and the Everglades

(54) Lake Hatchineha near Haines City, Fla.

Location.--Lat $28^{\circ} 00^{\prime} 00^{\prime \prime}$, long $81^{\circ} 22^{\prime} 50^{\prime \prime}$, in sec. 36, T. $28 \mathrm{~S} ., \mathrm{R}, 29 \mathrm{E}$, at the southeastern shore of lake, at head of Hatchineha-Kissimmee Canal, $3 \frac{1}{2}$ miles upstream from Lake Kissimmee, 14 miles east of Lake Wales, and 17 miles southeast of Haines City.

Records ava1lable.--U. S. Geological Survey: January 1942 to December 1953, da1ly gage helghts.

Gage.--Water-stage recorder. Datum of gage is $47.23 \mathrm{ft}$ above mean sea level, datum of 1929

(ievels by Corps of Engineers). Prior to June 8, 1950, at s1te on northwest shore of lake at same datum.

H1storical data.--Flood of June 1934 reached a stage of about $9.8 \mathrm{ft}$, from Information by local resident.

Annual peak stages

\begin{tabular}{|c|c|c|c|c|c|}
\hline Calendar year & Date & $\begin{array}{l}\text { Gage height } \\
\text { (feet) }\end{array}$ & Calendar year & Date & $\begin{array}{c}\text { Gage helght } \\
\text { feet) }\end{array}$ \\
\hline $\begin{array}{l}1934 \\
1942 \\
1943 \\
1944 \\
1945 \\
1946 \\
\end{array}$ & $\begin{array}{ll}\text { June } & - \\
\text { Mar. } & 20 \\
\text { Oct. } & 13 \\
\text { Oct. } & -\overline{1} \\
\text { Sept. } & 24,25 \\
\text { Oct. } & 8 \\
\end{array}$ & $\begin{array}{c}\text { about } 9.8 \\
\\
6.55 \\
5.01 \\
\text { a } 6.2 \\
8.89 \\
5.96 \\
\end{array}$ & $\begin{array}{l}1947 \\
1948 \\
1949 \\
1950 \\
1951 \\
1952 \\
1953 \\
\end{array}$ & $\begin{array}{l}\text { Oct. } 11 \\
\text { Oct. } 7,8 \\
\text { Oct. } 6 \\
\text { Jan. } 5,6,9 \\
\text { Nov. } 29 \\
\text { Oct. } 29 \\
\text { Oct. } 14,15,19 \\
\end{array}$ & $\begin{array}{l}9.56 \\
8.72 \\
8.08 \\
5.62 \\
5.77 \\
5.83 \\
9.61 \\
\end{array}$ \\
\hline
\end{tabular}

astimated.

Note.--Momentary peaks are wind-affected and gage helghts shown are maximum mean da1lies.

(55) Catf1sh Creek near Lake Wales, Fla.

Location.--Lat $27^{\circ} 57^{\prime} 40^{\prime \prime}$, long $81^{\circ} 29^{\prime} 48^{\prime \prime}$, in sec. 14, T. $29 \mathrm{~S} ., \mathrm{R} .28 \mathrm{E}$., on left bank $6 \mathrm{ft}$ downstream from bridge on private road, a quarter of a mile downstream from Lake Plerce, and 7 miles northeast of Lake Wales.

Drainage area. $--58.9 \mathrm{sq} \mathrm{ml}$.

Records ava1lable.--U. S. Geologlcal Survey: October 1947 to December 1953, da1ly gage he1ghts and discharges.

Gage.--Water-stage recorder. Datum of gage $1872.70 \mathrm{ft}$ above mean sea level (Corps of Engineers benchmark).

Stage-discharge relation.--Defined by current-meter measurements below 170 cfs. Considerable shifting at all stages.

Annual peak stages and discharges

\begin{tabular}{|c|c|c|c|c|c|c|c|}
\hline $\begin{array}{c}\text { Calendar } \\
\text { year }\end{array}$ & Date & $\begin{array}{c}\text { Gage } \\
\text { height } \\
\text { (feet) }\end{array}$ & $\begin{array}{c}\text { Discharge } \\
\text { (cfs) }\end{array}$ & $\begin{array}{c}\text { Calendar } \\
\text { year }\end{array}$ & Date & $\begin{array}{c}\text { Gage } \\
\text { height } \\
\text { (feet) }\end{array}$ & $\begin{array}{c}\text { Discharge } \\
\text { (cfs) }\end{array}$ \\
\hline $\begin{array}{l}1948 \\
1949 \\
1950 \\
\end{array}$ & $\begin{array}{ll}\text { Oct. } 3,4 \\
\text { Oct. } 2 \\
\text { Oct. } 25\end{array}$ & $\begin{array}{l}5.46 \\
5.32 \\
4.18 \\
\end{array}$ & $\begin{array}{r}180 \\
160 \\
68 \\
\end{array}$ & $\begin{array}{l}1951 \\
1952 \\
1953 \\
\end{array}$ & $\begin{array}{l}\text { Oct. } 2,3 \\
\text { Oct. } 27 \\
\text { Oct. } 9\end{array}$ & $\begin{array}{l}4.61 \\
- \\
5.81\end{array}$ & $\begin{array}{r}92 \\
113 \\
191\end{array}$ \\
\hline
\end{tabular}


Lake Okeechobee and the Everglades

(56) Lake Kissimmee near Lake Wales, Fla.

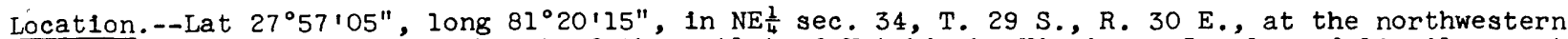
shore of lake, 1 mile southeast of the outlet of Hatchineha-Kissimmee Canal, and 16 miles east of Lake Wales.

Records ava1lable.--U. S. Geological Survey: March 1942 to December 1953, da1ly gage helghts.

Gage.--Water-stage recorder. Datum of gage is $46.94 \mathrm{ft}$ above mean sea level, datum of 1929 Tievels by Corps of Engineers). Prior to May 12, 1950, at site on southwest shore of lake and same datum.

Annual peak stages

\begin{tabular}{|c|c|c|c|c|c|}
\hline Calendar year & Date & $\begin{array}{l}\text { Gage he1ght } \\
\text { feet) }\end{array}$ & Calendar year & Date & $\begin{array}{c}\text { Gage helght } \\
\text { feet) }\end{array}$ \\
\hline $\begin{array}{l}1942 \\
1943 \\
1944 \\
1945\end{array}$ & $\begin{array}{ll}\text { Mar. } & 30 \\
\text { Oct. } & 14 \\
\text { Nov. } & 11,12 \\
\text { Sept. } & 18-22\end{array}$ & $\begin{array}{l}6.22 \\
4.45 \\
5.32 \\
8.80\end{array}$ & $\begin{array}{l}1948 \\
1949 \\
1950\end{array}$ & $\begin{array}{ll}\text { Oct. } & 6-8 \\
\text { Oct. } & 5-7 \\
\text { Jan. } & 1 \\
& \end{array}$ & $\begin{array}{l}8.78 \\
7.70 \\
4.95\end{array}$ \\
\hline $\begin{array}{l}1946 \\
1947\end{array}$ & $\begin{array}{l}\text { Oct. } 13 \\
\text { Sept. }\end{array}$ & $\begin{array}{l}5.32 \\
9.77\end{array}$ & $\begin{array}{l}1952 \\
1953\end{array}$ & $\begin{array}{l}\text { Nov: } 1 \\
\text { Oct. } 12,13\end{array}$ & $\begin{array}{l}5.70 \\
9.70\end{array}$ \\
\hline
\end{tabular}

Note.--Momentary peaks are wind-affected and gage helghts shown are maximum mean dallies except that for September 1947.

(57) Weohyakapka-Rosalle Canal near Lake Wales, Fla.

Location.--Lat $27^{\circ} 52^{\prime}$, long $81^{\circ} 23^{\prime}$, in sec. 14, T. $30 \mathrm{~S} ., \mathrm{R} .29 \mathrm{E}$, near left bank at downstream side of bridge on State Highway 60 , $11 \frac{1}{2}$ miles east of Lake Wales.

Records avallable.--U. S. Geological Survey: August 1942 to August 1948, da1ly gage helghts and perlodic discharge measurements.

Gáge.--Water-stage recorder. Datum of gage $1 \mathrm{~s} 55.23 \mathrm{ft}$ above mean sea level (Corps of Engineers benchmark).

Stage-discharge relation.--Not defined; affected by backwater from Lake Rosalie.

H1storical data.--Flood of September 1933 reached a stage of $7.6 \mathrm{ft}$, from floodmarks on piles and information by a local resident.

Annual peak stages

\begin{tabular}{|c|c|c|c|c|c|}
\hline Calendar year & Date & $\begin{array}{l}\text { Gage helght } \\
\text { feet) }\end{array}$ & Calendar year & Date & $\begin{array}{l}\text { Gage helght } \\
\text { feet) }\end{array}$ \\
\hline 1933 & Sept. - & 7.6 & 1945 & Sept. 18 & 5.99 \\
\hline $\begin{array}{l}1943 \\
1944\end{array}$ & $\begin{array}{l}\text { Oct. } 11 \\
\text { Sept. } 15,18\end{array}$ & $\begin{array}{l}3.38 \\
4.40\end{array}$ & $\begin{array}{l}1946 \\
1947\end{array}$ & $\begin{array}{l}\text { Aug. } \quad 4-6 \\
\text { Sept. } \quad 24\end{array}$ & $\begin{array}{l}4.23 \\
6.21\end{array}$ \\
\hline
\end{tabular}


Lake Okeechobee and the Everglades

(58) K1ssimmee R1ver below Lake K1ss1mmee, Fla.

Locat1on.--Lat $27^{\circ} 46^{\prime} 13^{\prime \prime}$, long $81^{\circ} 10^{\prime} 45^{\prime \prime}$, 1n sec. 24, T. 31 S., R. 31 E., on r1ght bank about 3 m1les downstream from Lake K1ss1mmee and bridge on State H1ghway 60 , and 22 m1les east of Frostproor.

Drainage area.--1,609 $\mathrm{sq} \mathrm{ml}$ at State Highway 60 (1ncludes areas dra1ned by Lake Weohyakapka and Lake Mar1an).

Records avallable.--Okeechobee Flood Control D1strlct: January 1930 to June 1931, da1ly gage helghts. U. S. Geological Survey: June 1931 to December 1953, dally gage helghts and discharges (records unpublished prior to October 1933).

Gage.--Water-stage recorder. Datum of gage $1 \mathrm{~s} 43.48 \mathrm{ft}$ above mean sea levèl, datum of 1929 (levels by Corps of Englneers). Prior to Mar. 21, 1934, staff gage at bridge 3 miles upstream at datum $44.73 \mathrm{ft}$ lower. Mar. 21, 1934, to Sept. 30, 1950, water-stage recorder at present site at datum $45.00 \mathrm{ft}$ lower than present datum. Since Mar. 21, 1934, gage at bridge $3 \mathrm{miles}$ upstream used as supplemental gage. (All gage helghts shown below have been reduced to mean sea level).

Stage-d1scharge relation.--Defined by current-meter measurements. Cons1derable shifting at all stages caused by dikes and levees in the flood pla1n below the station.

Annual peak stages and discharges

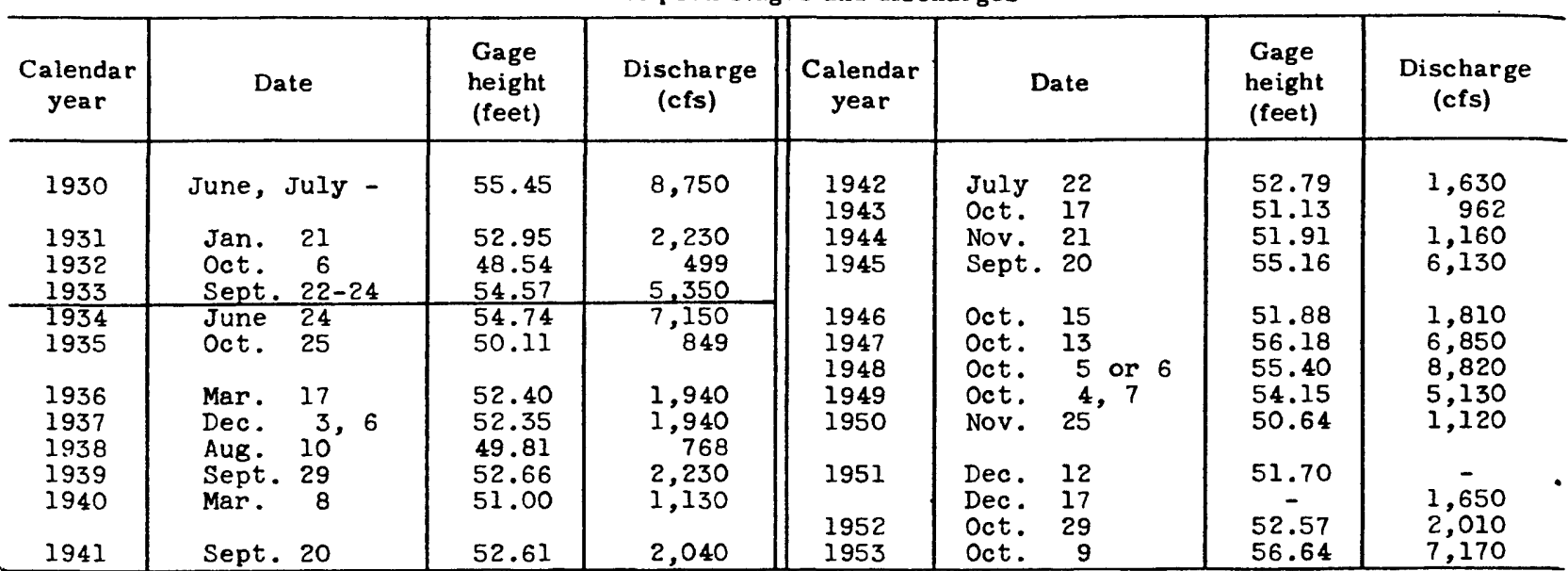

(59) K1ssimmee R1ver at Fort K1ss1mmee, Fla.

Location.--Lat $27^{\circ} 35^{\prime}$, long $81^{\circ} \mathrm{O} 9^{\prime}$, in sec. 19, T. $33 \mathrm{~S} ., \mathrm{R}$. 32 E., on right bank at Fort Kissimmee, 21 miles east of Avon Park.

Records avallable.--U. S. Geological Survey: December 194l to December 1953 , da1ly gage he1ghts.

Gage.--Water-stage recorder. Datum of gage $1 \mathrm{~s} 38.03 \mathrm{ft}$ above mean sea level, datum of 1929 (levels by Corps of Englneers). Dec. 9, 1941, to Feb. 25, 1942, staff gage at s1te 500 ft downstream at different datum.

Stage-d1scharge relat1on.--Not def1ned. 
Lake Okeechobee and the Everglades

(59) Kissimmee River at Fort Kissimmee, Fla.--Continued

Annual peak stages

\begin{tabular}{|c|c|c|c|c|c|}
\hline Calendar year & Date & $\begin{array}{l}\text { Gage height } \\
\text { (feet) }\end{array}$ & Calendar year & Date & $\begin{array}{l}\text { Gage he Ight } \\
\text { (feet) }\end{array}$ \\
\hline $\begin{array}{l}1942 \\
1943 \\
1944 \\
1945\end{array}$ & $\begin{array}{ll}\text { June } & 30 \\
\text { Oct. } & 11,12 \\
\text { Oct. } & 25,26 \\
\text { Sept. } & 18\end{array}$ & $\begin{array}{r}7.99 \\
7.21 \\
7.48 \\
12.17\end{array}$ & $\begin{array}{l}1948 \\
1949 \\
1950\end{array}$ & $\begin{array}{lr}\text { Sept. } & 24 \\
\text { Oct. } & 1 \\
\text { Oct. } & -\end{array}$ & $\begin{array}{c}11.97 \\
010.24 \\
7.4\end{array}$ \\
\hline $\begin{array}{l}1946 \\
1947 \\
\end{array}$ & $\begin{array}{l}\text { Sept. } 22 \\
\text { Sept. } 23\end{array}$ & $\begin{array}{r}8.29 \\
11.84 \\
\end{array}$ & $\begin{array}{l}1952 \\
1953\end{array}$ & $\begin{array}{ll}\text { Oct. } 23 \\
\text { Oct. } 11\end{array}$ & $\begin{array}{r}9.07 \\
12.89 \\
\end{array}$ \\
\hline
\end{tabular}

a From fragmentary gage-height record; peak may have been slightly higher.

(60) Reedy Lake Outlet near Frostproof, Fla.

Location.--Lat $27^{\circ} 43^{\prime} 13^{\prime \prime}$, long $81^{\circ} 28^{\prime} 40^{\prime \prime}$, in NW/ sec. 1, T. $32 \mathrm{~S} ., \mathrm{R}$. 28 E., on left bank $15 \mathrm{ft}$ upstream from county road bridge, $100 \mathrm{ft}$ downstream from Reedy Lake, and $3 \frac{1}{2}$ miles southeast of Frostproof.

Drainage area. $--62.2 \mathrm{sq} \mathrm{m} 1$.

Records avallable.--U. S. Geological Survey: October 1946 to December 1953, da1ly gage helghts and discharges.

Gage.--Water-stage recorder and concrete control. Datum of gage is 76.05 ft above mean sea level, datum of 1929 .

Stage-discharge relation.--Defined by current-meter measurements. Considerable shifting as the result of changes in the elevation of the control and aquatic vegetation.

Annual peak stages and discharges

\begin{tabular}{c|c|c|c||c|c|c|c}
\hline $\begin{array}{c}\text { Calendar } \\
\text { year }\end{array}$ & Date & $\begin{array}{c}\text { Gage } \\
\text { height } \\
\text { (feet) }\end{array}$ & $\begin{array}{c}\text { Discharge } \\
\text { (cfs) }\end{array}$ & $\begin{array}{c}\text { Calendar } \\
\text { year }\end{array}$ & Date & $\begin{array}{c}\text { Gage } \\
\text { height } \\
\text { (feet) }\end{array}$ & $\begin{array}{c}\text { Discharge } \\
\text { (cfs) }\end{array}$ \\
\hline 1947 & Sept. 29 & 4.00 & 141 & 1951 & Oct. 2 & 2.85 & 57 \\
1948 & Oct. 5 & 4.37 & 166 & 1952 & Oct. 21 & 2.48 & 40 \\
1949 & Oct. 2 & 3.49 & 107 & 1953 & Oct. 9 & 4.09 & 116 \\
1950 & oct. 21-24 & - & 54 & & \\
\hline
\end{tabular}




\section{Lake Okeechobee and the Everglades}

(61) Lake Arbuckle near Avon Park, Fla.

Location.--Lat $27^{\circ} 39^{\prime} 50^{\prime \prime}$, long $81^{\circ} 22^{\prime} 40^{\prime \prime}$, in SW sec. 25, T. $32 \mathrm{~S}$, R. 29 E., on downstream s1de of bridge across Arbuckle Creek at south side of lake, and 9 miles northeast of Avon Park.

Records avallable.--U. S. Geological Survey: December 1941 to December 1953, daily gage heights. Gage.--Staff gage read once da1ly. Datum of gage $1851.53 \mathrm{ft}$ above mean sea level, datum of 1929.

H1storical data.--Hurricane floods of 1926 and 1928 reached a stage of $7.2 \mathrm{ft}$.

Annual peak stages

\begin{tabular}{|c|c|c|c|c|c|}
\hline Calendar year & Date & $\begin{array}{c}\text { Gage helght } \\
\text { feet) }\end{array}$ & Calendar year & Date & $\begin{array}{l}\text { Gage helght } \\
\text { feet) }\end{array}$ \\
\hline $\begin{array}{l}1926 \\
1928\end{array}$ & $\begin{array}{l}- \\
\operatorname{Man} \\
10\end{array}$ & $\begin{array}{l}7.2 \\
7.2 \\
3.56\end{array}$ & $\begin{array}{l}1947 \\
1948 \\
1949 \\
1950\end{array}$ & $\begin{array}{l}\text { Sept. } 24 \\
\text { Sept. } 24 \\
\text { Aug. } 30, \text { oct. } 1 \\
\text { Oct. } 21,22\end{array}$ & $\begin{array}{l}6.14 \\
6.7 \\
5.80 \\
5.00\end{array}$ \\
\hline $\begin{array}{l}1942 \\
1943 \\
1944 \\
1945\end{array}$ & $\begin{array}{l}\text { Mar. } 19 \\
\text { Sept. } 1,2 \\
\text { oct. } 27,28 \\
\text { Sept. } 17\end{array}$ & $\begin{array}{l}3.56 \\
4.20 \\
3.62 \\
6.20\end{array}$ & $\begin{array}{l}1951 \\
1952 \\
1953\end{array}$ & $\begin{array}{ll}\text { Oct. } & 6 \\
\text { Oct. } & 24,25 \\
\text { Oct. } & 10,11\end{array}$ & $\begin{array}{l}4.50 \\
5.00 \\
6.20\end{array}$ \\
\hline 1946 & Sept. 21 & 4.32 & & & \\
\hline
\end{tabular}

(62) Arbuckle Creek near De Soto C1ty, Fla.

Location.--Lat $27^{\circ} 26^{\prime} 33^{\prime \prime}$, long $81^{\circ} 17^{\prime} 51^{\prime \prime}$, in SE⿺ sec. 11, T. 35 S., R. 30 E., on right bank $20 \mathrm{ft}$ downstream from bridge on State H1ghway $700,1 \mathrm{mlle}$ upstream from Lake Istokpoga, and $7 \mathrm{mlles}$ east of De Soto C1ty.

Dra1nage area.--385 sq $\mathrm{ml}$ (excludes area drained by Lake Weohyakapka and inclühes area drained by Lake Sebring).

Records avallable.--U. S. Geological Survey: June 1939 to December 1953, da1ly gage helghts and discharges.

Gage.--Water-stage recorder. Datum of gage $1835.51 \mathrm{ft}$ above mean sea level, datum of 1929. June 24,1942 , to Oct. 19, 1943, staff gage at same site and datum.

Stage-d1scharge relation.--Poorly defined by current-meter measurements below 5,300 cf 8 ; affected by backwater from Lake Istokpoga.

Remarks.--Records include flow through 2 overflow bridges, 1.9 and 2.1 miles west of main channel. 
Lake Okeechobee and the Everglades

(62) Arbuckle Creek near De Soto City, Fla.--Continued

Annual peak stages and discharges

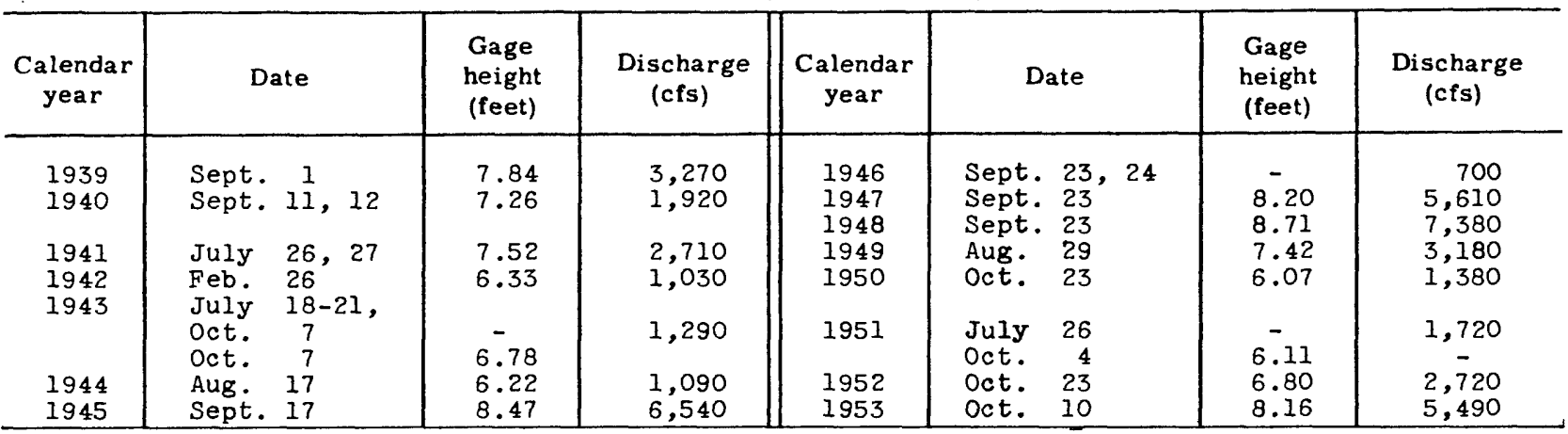

(63) Josephine Creek near De Soto C1ty, Fla.

Location.--Lat $27^{\circ} 22^{\prime} 26^{\prime \prime}$, Iong $81^{\circ} 23^{\prime} 37^{\prime \prime}$, In SE⿺ sec. 2, T. 36 S., R. 29 E., on left bank $320 \mathrm{ft}$ downstream from bridge on State Highway 17, 1 mile downstream from Jack Creek, and 4 miles south of De Soto City.

Dra1nage area.--109 sq mi (excludes area dralned by Lake Sebring).

Records ava1lable.--U. S. Geological Survey: October 1946 to December 1953, da1ly gage helghts and discharges.

Gage.--Water-stage recorder. Datum of gage is $52.99 \mathrm{ft}$ above mean sea level, datum of 1929 (State Road Department benchmark). Prior to May 21, 1952, at site half a mile upstream at datum $0.89 \mathrm{ft}$ higher.

Stage-d1scharge relation.--Defined by current-meter measurements.

Annual peak stages and discharges

\begin{tabular}{|c|c|c|c|c|c|c|c|}
\hline $\begin{array}{c}\text { Calendar } \\
\text { year }\end{array}$ & Date & $\begin{array}{l}\text { Gage } \\
\text { height } \\
\text { (feet) }\end{array}$ & $\begin{array}{l}\text { Discharge } \\
\text { (cfs) }\end{array}$ & $\begin{array}{c}\text { Calendar } \\
\text { year }\end{array}$ & Date & $\begin{array}{l}\text { Gage } \\
\text { height } \\
\text { (feet) }\end{array}$ & $\begin{array}{l}\text { Discharge } \\
\text { (cfs) }\end{array}$ \\
\hline $\begin{array}{l}1947 \\
1948 \\
1949 \\
1950\end{array}$ & $\begin{array}{ll}\text { Sept. } & 19 \\
\text { Sept. } & 23 \\
\text { Aug. } & 28 \\
\text { Oct. } & 18\end{array}$ & $\begin{array}{r}9.55 \\
11.56 \\
9.31 \\
5.81 \\
\end{array}$ & $\begin{array}{r}900 \\
1,780 \\
792 \\
88\end{array}$ & $\begin{array}{l}1951 \\
1952 \\
1953\end{array}$ & $\begin{array}{ll}\text { Oct. } & 2 \\
\text { Oct. } 21 \\
\text { Oct. } 10\end{array}$ & $\begin{array}{l}\frac{8.30}{5.55} \\
7.97\end{array}$ & $\begin{array}{r}487 \\
184 \\
1,180\end{array}$ \\
\hline
\end{tabular}


Lake Okeechobee and the Everglades

(64) Lake Istokpoga near De Soto C1ty, Fla.

Location.--Lat $27^{\circ} 26^{\prime} 05^{\prime \prime}$, long $81^{\circ} 17^{\prime} 05^{\prime \prime}$, in sec. 13, T. 35 S., R. 30 E., near north shore of lake, about $500 \mathrm{ft}$ east of mouth of Arbuckle Creek, $i$ mile southeast of bridge on $U$. $S$. H1ghway 98 crossing Arbuckle Creek, and 7 miles east of De Soto C1ty.

Records avallable.--Corps of Englneers: August 1936 to August 1942, dally gage helghts. U. S. Geolog1cal Survey: August 1942 to December 1953, da1ly gage helghts.

Gage.--Water-stage recorder. Datum of gage $1834.07 \mathrm{ft}$ above mean sea level, datum of 1929 . Prior to Aug. 20, 1942, Corps of Englneers staff gage on west shore of lake was read once da1ly. Aug. 11, 1936, to May 18, 1937, datum of gage was $40.54 \mathrm{ft}$ above mean sea level, datum of 1929, and May 19, 1937, to Aug. 19, 1942, datum was $38.54 \mathrm{ft}$ above mean sea level, datum of 1929. (All gage helghts shown below have been reduced to elevations above mean sea level).

Annual peak stages

\begin{tabular}{|c|c|c|c|c|c|}
\hline Calendar year & Date & $\begin{array}{c}\text { Elevation, } \\
\text { feet above } \\
\text { mean sea level }\end{array}$ & Calendar year & Date & $\begin{array}{c}\text { Elevation, } \\
\text { feet above } \\
\text { mean sea level }\end{array}$ \\
\hline $\begin{array}{l}1937 \\
1938 \\
1939 \\
1940\end{array}$ & $\begin{array}{ll}\text { Dec. } & 9-21 \\
\text { Aug. } & \overline{6} \\
\text { Oct. } & 6 \\
\text { Sept. } & 29 \\
\text { Oct. } & 3\end{array}$ & $\begin{array}{l}39.4 \\
(a) \\
41.2 \\
41.6\end{array}$ & $\begin{array}{l}1946 \\
1947 \\
1948 \\
1949 \\
1950\end{array}$ & $\begin{array}{lr}\text { Jan. } & 6 \\
\text { Sept. } & 23 \\
\text { Sept. } & 24 \\
\text { Oct. } & 11 \\
\text { Jan. } \quad 4-6\end{array}$ & $\begin{array}{l}39.79 \\
42.47 \\
42.44 \\
40.63 \\
38.77\end{array}$ \\
\hline $\begin{array}{l}1941 \\
1942 \\
1943 \\
1944 \\
1945\end{array}$ & $\begin{array}{lc}\text { Aug. } & 2-9 \\
\text { July } & 24-28 \\
\text { Oct. } & 11 \\
\text { Jan. } & 2 \\
\text { Sept. } & -\end{array}$ & $\begin{array}{l}41.0 \\
40.4 \\
41.38 \\
39.16 \\
43.22\end{array}$ & $\begin{array}{l}1951 \\
1952 \\
1953\end{array}$ & $\begin{array}{l}\text { Apr. } \\
\text { Nov. } \\
\text { Oct. } \\
12\end{array}$ & $\begin{array}{l}39.51 \\
39.52 \\
42.27\end{array}$ \\
\hline
\end{tabular}

a No gage-he1ght record.

Note.--Momentary peaks are wind-affected and gage helghts shown are maximum mean da1lies except that for september 1945 .

(65) Istokpoga Canal near Cornwell, Fla.

Location.--Lat $27^{\circ} 24^{\prime} 01^{\prime \prime}$, long $81^{\circ} 09^{\prime} 35^{\prime \prime}$, 1n sec. 30, T. 35 S., R. 32 E., on right bank $30 \mathrm{ft}$ downstream from old highway bridge, a quarter of a mile downstream from Seaboard Air Line Rallroad bridge, $1 \frac{1}{2}$ miles upstream from K1ssimmee River, and $4 \frac{1}{2}$ miles northwest of Cornwell Post Office.

Drainage area. $--624 \mathrm{sq} \mathrm{m}$.

Records avallable.--U. S. Geolog1cal Survey: March 1934 to December 1953, da1ly gage helghts and discharges.

Gage.--Water-stage recorder. Datum of gage $1 \mathrm{~s} 29.71 \mathrm{ft}$ above mean sea level (levels by Corps of Englneers). May 15, 1942, to Aug. 19, 1949, staff gage at same site and datum.

Since June 3, 1953, auxiliary water-stage recorder, below control, $1 \frac{3}{4}$ miles upstream.

Stage-discharge relation.--Defined by current-meter measurements; affected by backwater from K1ssimmee River. Since June 1953, discharges computed by using fall as determined from aux1l1ary water-stage recorder, as a factor.

H1storical data.--Flood of September 1933 reached a stage of $10.1 \mathrm{ft}$.

Remarks.--Some diversions at times during high water from Lake Istokpoga into Indian Prairie and Harney Pond Canals when levees on southeast shore of lake are overtopped or washed out. 
Lake Okeechobee and the Everglades

(65) Istokpoga Canal near Cornwell, Fla.--Continued

Annual peak stages and discharges



(66) K1ssimmee River near Cornwell, Fla.

Location.--Lat $27^{\circ} 21^{\prime} 44^{\prime \prime}$, long $81^{\circ} 03^{\prime} 03^{\prime \prime}$, in sec. $8, \mathrm{~T} .36 \mathrm{~S}$., R. $33 \mathrm{E}$, on right bank 1 , 000 ft downstream from new Pearce Bridge on U. S. Highway 98 and State Highway 700,3 m1les southeast of Cornwell Post office, and $10 \frac{1}{2}$ miles downstream from Istokpoga Canal.

Drainage area. $--2,703 \mathrm{sq} \mathrm{m} 1$.

Records ava1lable.--Everglades Drainage District: August 1928 to December 1930, da1ly gage helghts. Okeechobee Flood Control District: January to June 1931, daily gage helghts. U. S. Geological Survey: June 1931 to December 1953, dally gage helghts. October 1948 to September 1951, da1ly discharges.

Gage.--Staff gage read twice da1ly. Datum of gage is $24.64 \mathrm{ft}$ above mean sea level (Corps of Englneers benchmark). Prior to $\mathrm{Feb}$. 14, 1934, at same site at datum $5.36 \mathrm{ft}$ below mean sea level. (All gage heights shown below have been reduced to elevations above mean sea level).

Stage-discharge relation.--Relation for use October 1948 to September 1951 was defined by current-meter measurements below $8,500 \mathrm{cfs}$. Backwater effect and shifting channel preclude computation of discharge maxima for other years. 
Lake Okeechobee and the Everglades

(66) Kissimmee River near Cornwell, Fla.--Continued

Annual peak stages and discharges

\begin{tabular}{|c|c|c|c|c|c|c|c|}
\hline $\begin{array}{c}\text { Calendar } \\
\text { year }\end{array}$ & Date & $\begin{array}{c}\text { Gage } \\
\text { height } \\
\text { (feet) }\end{array}$ & $\begin{array}{c}\text { Discharge } \\
\text { (cfs) }\end{array}$ & $\begin{array}{c}\text { Calendar } \\
\text { year }\end{array}$ & Date & $\begin{array}{l}\text { Gage } \\
\text { height } \\
\text { (feet) }\end{array}$ & $\begin{array}{c}\text { Discharge } \\
\text { (cfs) }\end{array}$ \\
\hline $\begin{array}{l}1928 \\
1929 \\
1930 \\
1931 \\
1932 \\
1933 \\
1934 \\
1935 \\
1936 \\
1937 \\
1938 \\
1939 \\
1940\end{array}$ & $\begin{array}{l}\text { Aug. } 11,12 \\
\text { Oct. } 31, \\
\text { June } 23,24 \\
\text { Mar. } 11 \\
\text { Sept. } 14 \\
\text { Sept. } 5 \\
\text { June } 22 \\
\text { Oct. } 15,16 \\
\text { Mar. } 15-18 \\
\text { Oct. } 24 \\
\text { Jan. } 8 \\
\text { Sept. } 26 \\
\text { Oct. } 6,7\end{array}$ & $\begin{array}{l}35.9 \\
33.1 \\
34.8 \\
32.4 \\
31.40 \\
.33 .64 \\
33.88 \\
31.92 \\
32.10 \\
33.26 \\
31.29 \\
32.40 \\
31.84\end{array}$ & $\begin{array}{l}- \\
- \\
- \\
- \\
- \\
- \\
- \\
- \\
-\end{array}$ & $\begin{array}{l}1941 \\
1942 \\
1943 \\
1944 \\
1945 \\
1946 \\
1947 \\
1948 \\
1949 \\
1950 \\
1951 \\
1952 \\
1953\end{array}$ & $\begin{array}{l}\text { Aug. } \quad 3 \\
\text { Feb. } \quad 25,26 \\
\text { Oct. } \quad 5 \\
\text { Nov. } \quad 5-7 \\
\text { Sept. } 22 \\
\text { Jan. } \quad 1,2 \\
\text { Sept. } \quad 24 \\
\text { Oct. } \quad 6 \\
\text { Oct. } \quad 5 \\
\text { Oct. } \quad 26 \\
\text { Oct. } \quad 3 \\
\text { Oct. } \quad 28 \\
\text { Oct. } \quad 12,13\end{array}$ & $\begin{array}{l}32.26 \\
32.32 \\
32.22 \\
30.66 \\
34.64 \\
31.22 \\
34.90 \\
35.45 \\
33.20 \\
31.64 \\
32.80 \\
32.74 \\
36.12\end{array}$ & $\begin{array}{c}- \\
- \\
- \\
- \\
- \\
- \\
16,800 \\
8,600 \\
4,000 \\
- \\
- \\
-\end{array}$ \\
\hline
\end{tabular}

(67) K1ssimmee R1ver near Okeechobee, Fla.

Location.-Iat $27^{\circ} 14^{\prime} 18^{\prime \prime}$, long $80^{\circ} 58^{\prime} 57^{\prime \prime}$, in sec. 24 , T. 37 S., R. 33 E., on downstream end of left pler of bridge on state H1ghway $70,9.4 \mathrm{miles}$ west of okeechobee, and approximately 13 miles upstream from Lake Okeechobee.

Drainage area. $--2,886 \mathrm{sq} \mathrm{ml}$.

Records ava1lable.--Everglades Drainage District: August 1928 to December 1930 , da1ly gage helghts. U.S. Geological Survey: January 1931 to December 1953, da1ly gage helghts and discharges (records unpublished prior to October 1930).

Gage.-Water-stage recorder. Datum of gage is 1.37 f.t below mean sea level, datum of 1929 . Prior to Apr. 28, 1949, staff gage at same site and datum.

Stage-discharge relation.--Defined by current-meter measurements below $14,000 \mathrm{cfs}$. Cons1derable shifting at all stages.

Annual peak stages and discharges

\begin{tabular}{|c|c|c|c|c|c|c|c|}
\hline $\begin{array}{c}\text { Calendar } \\
\text { year }\end{array}$ & Date & $\begin{array}{c}\text { Gage } \\
\text { height } \\
\text { (feet) }\end{array}$ & $\begin{array}{c}\text { Discharge } \\
\text { (cfs) }\end{array}$ & $\begin{array}{c}\text { Calendar } \\
\text { year }\end{array}$ & Date & $\begin{array}{c}\text { Gage } \\
\text { height } \\
\text { (feet) }\end{array}$ & $\begin{array}{c}\text { Discharge } \\
\text { (cfs) }\end{array}$ \\
\hline $\begin{array}{l}1928 \\
1929 \\
1930 \\
1931 \\
1932 \\
1933 \\
1934 \\
1935 \\
1936 \\
1937 \\
1938 \\
1939 \\
1940\end{array}$ & $\begin{array}{l}\text { Aug. } \\
\text { Oct. } \\
\text { June } 23,24 \\
\text { Mar. } \quad 8-10 \\
\text { Sept. } 16 \\
\text { Sept. } 9 \\
\text { June } 24 \\
\text { Oct. } 13 \\
\text { Mar. } 16,17 \\
\text { Oct. } 27 \\
\text { Jan. } 9-13 \\
\text { Sept. } 27 \\
\text { Oct. } 8 \\
\end{array}$ & $\begin{array}{l}30.3 \\
\alpha 27.0 \\
28.7 \\
26.2 \\
25.70 \\
29.32 \\
27.72 \\
26.04 \\
25.84 \\
26.90 \\
24.88 \\
26.56 \\
25.54\end{array}$ & $\begin{array}{r}20,000 \\
7,260 \\
13,000 \\
3,910 \\
3,210 \\
15,600 \\
9,000 \\
4,330 \\
3,270 \\
6,080 \\
2,320 \\
5,150 \\
3,360 \\
\end{array}$ & $\begin{array}{l}1941 \\
1942 \\
1943 \\
1944 \\
1945 \\
1946 \\
1947 \\
1948 \\
1949 \\
1950 \\
1951 \\
1952 \\
1953 \\
\end{array}$ & \begin{tabular}{|cc} 
July 31 to Aug. 5 \\
Feb. 27,28 \\
Oct. 7 \\
Nov. $10-14$ \\
Sept. 22 \\
Sept. 20 \\
Sept. 25 \\
Oct. \\
Oct. $\quad 6$ \\
Oct. 28 \\
Oct. & 4 \\
Oct. & 29 \\
Oct. & 14 \\
\end{tabular} & $\begin{array}{l}25.98 \\
26.20 \\
26.28 \\
23.88 \\
27.90 \\
24.88 \\
28.42 \\
29.34 \\
26.84 \\
24.92 \\
26.25 \\
25.72 \\
28.37\end{array}$ & $\begin{array}{r}4,280 \\
4,650 \\
4,840 \\
1,840 \\
11,700 \\
2,970 \\
13,000 \\
17,400 \\
8,300 \\
3,740 \\
7,280 \\
5,750 \\
17,800 \\
\end{array}$ \\
\hline
\end{tabular}

- Result of discharge measurement; peak may have been slightly higher.

Note.--Gage helghts shown have not been reduced to elevations above mean sea level. 
Lake Okeechobee and the Everglades

(68) St. Luc1e Canal at Lake Okeechobee, Fla.

Location.--Lat $26^{\circ} 59^{\prime}$, long $80^{\circ} 37^{\prime}$, in sec. 22 , T. $40 \mathrm{~S} .$, R. $37 \mathrm{E}$, on downstream side of left pler of bridge on State H1ghway' 15 at Lake Okeechobee, and 23.9 miles upstream from lock near stuart.

Records avallable.--U. S. Geological Survey: Apr1l 1931 to September 1952, da1ly gage helghts and discharges.

Gage.--Water-stage recorder. Datum of gage is at mean sea level, datum of 1929 (Corps of Englneers benchmark). Prior to Jan. 17, 1934, staff gage at site a quarter of a m1le downstream at datum $i .13 \mathrm{ft}$ lower. Jan. 17, 1934, to Mar. 16, 1951, water-stage recorder at Florlda East Coast Ra1lway bridge, 0.7 mile downstream at datum $1.56 \mathrm{ft}$ lower. (All gage helghts tabulated below have been reduced to present datum, at mean sea level).

Since Jan. 17, 1934, aux1liary water-stage recorder $10.8 \mathrm{miles}$ downstream.

Stage-discharge relation.--Defined by current-meter measurements; affected by fall. Discharge computed by using fall, as determined by aux1liary water-stage recorder, as a factor.

Remarks.--Prior to Mar. 1, 194.2, flow regulated by lock 1. Subsequent to Mar. 1, 1942, flow regulated by lock near stuart.

Annual peak stages and discharges

\begin{tabular}{|c|c|c|c|c|c|c|c|}
\hline $\begin{array}{c}\text { Calendar } \\
\text { year }\end{array}$ & Date & $\begin{array}{c}\text { Dally gage } \\
\text { helght } \\
\text { (feet above } \\
\text { mean } \\
\text { sea level) }\end{array}$ & $\begin{array}{c}\text { Discharge } \\
\text { (cfs) }\end{array}$ & $\begin{array}{l}\text { Calendar } \\
\text { year }\end{array}$ & Date & $\begin{array}{c}\text { Da1ly gage } \\
\text { helght } \\
\text { (feet above } \\
\text { mean } \\
\text { sea level) }\end{array}$ & $\begin{array}{l}\text { D1scharge } \\
\text { (cfs) }\end{array}$ \\
\hline $\begin{array}{l}1931 \\
1932 \\
1933\end{array}$ & $\begin{array}{l}\text { Sept. } 29 \\
\text { Oct. } 25\end{array}$ & $\begin{array}{c}\text { (a) } \\
- \\
14.93\end{array}$ & $\begin{array}{c}(a) \\
2,820 \\
4,740\end{array}$ & 1943 & $\begin{array}{lc}\text { Jan. } & 2 \\
\text { Oct. } & 26 \\
\text { Nov. } & 29\end{array}$ & - & 1,050 \\
\hline 1934 & $\begin{array}{ll} & \\
\text { Oct. } & 4 \\
\text { Oct. } & 8\end{array}$ & & & 1944 & $\begin{array}{lc}\text { Dec: } & 12 \\
\text { Jan. } & 3\end{array}$ & $\begin{array}{l}13.78 \\
13.73\end{array}$ & \\
\hline 1935 & $\begin{array}{ll}\text { Oct. } & 15 \\
\text { Oct. } & 29\end{array}$ & 14.44 & $4, \overline{500}$ & 1945 & $\begin{array}{l}\text { Nov. } 11 \\
\text { Oct. } 25,26 \\
\text { Dec. } 31\end{array}$ & $\begin{array}{c}- \\
- \\
15.51\end{array}$ & $\begin{array}{r}591 \\
6,120 \\
\end{array}$ \\
\hline 1936 & $\begin{array}{ll}\text { Mar. } & 17 \\
\text { July } & 13\end{array}$ & 14.51 & 5,020 & 1946 & Feb. 24 & (b) & 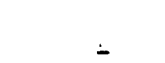 \\
\hline 1937 & $\begin{array}{ll}\text { Dec. } & 4 \\
\text { Dec. } & 6\end{array}$ & $\begin{array}{l}14.74 \\
-\end{array}$ & $5, \overline{0} 70$ & 1947 & & 18.04 & \\
\hline 1938 & $\begin{array}{r}\text { Jan. } \quad 2 \\
\text { Jan. } 16\end{array}$ & 14.19 & 4,690 & 1948 & $\begin{array}{l}\text { Oct. } 18 \\
\text { Oct. } 22-24\end{array}$ & 17.10 & $6, \overline{110}$ \\
\hline $\begin{array}{l}1939 \\
1940\end{array}$ & $\begin{array}{ll}\text { Oct. } & 7 \\
\text { Nov. } & 8 \\
\text { Aor. } & 8\end{array}$ & 14.50 & $\begin{array}{c}3,490 \\
- \\
-\end{array}$ & 1949 & $\begin{array}{l}\text { Sept. } 2-5 \\
\text { Nov. } 25 \\
\text { Jan. }\end{array}$ & 14.99 & 6,700 \\
\hline & oct. 1,2 & $+x$ & 4,500 & 1951 & oct. & 15.73 & - \\
\hline 1941 & $\begin{array}{ll}\text { Sept. } & 29 \\
\text { Oct. } & 23 \\
\text { Mar. } & 3\end{array}$ & $\begin{array}{l}- \\
14.60 \\
14.55\end{array}$ & $\begin{array}{l}4,040 \\
-\overline{170}\end{array}$ & 1952 & Nov. $\quad 2,6$ & $\begin{array}{l}10.10 \\
\text { (a) }\end{array}$ & $\begin{array}{c}7,470 \\
(a)\end{array}$ \\
\hline
\end{tabular}

a Incomplete records for year.

b No gage-height record.

c Lock closed ent1re year; discharge less than 10 cfs. 


\section{Lake Okeechobee and the Everglades}

(69) West Palm Beach Canal at Canal Point, Fla.

Location.--Iat $26^{\circ} 52^{\prime}$, long $80^{\circ} 38^{\prime}$, In sec. 34, T. 41 S., R. 37 E., at upstream end of lock at right bank in Canal Polnt, $350 \mathrm{ft}$ downstream from state Highway 15 and 550 ft downstream from outlet from Lake Okeechobee.

Records ava1lable.--U. S. Geological Survey: November 1939 to December 1953, da1ly gage helghts and discharges.

Gage.--Staff gage read three times a day. Datum of gage 1s at mean sea level, datum of 1929. Since May 1940, auxillary water-stage recorder below lock and dam.

Stage-discharge relation.- There is no definite relation between stage and discharge at this station due to manipuiation of boards in the spillway dam and because of various amounts of submergence. Ratings are based on relations between velocity at a point and mean velocity and between stage and area as defined by current-meter measurements. Velocity at a point observed three times a day.

Remarks.--Flow regulated at station by manipulation of stoplogs in lock and dam and hurricane gates. Flow occasionally reversed after periods of considerable rainfall owing to downstream natural dralnage and to pumping from agricultural lands in Everglades.

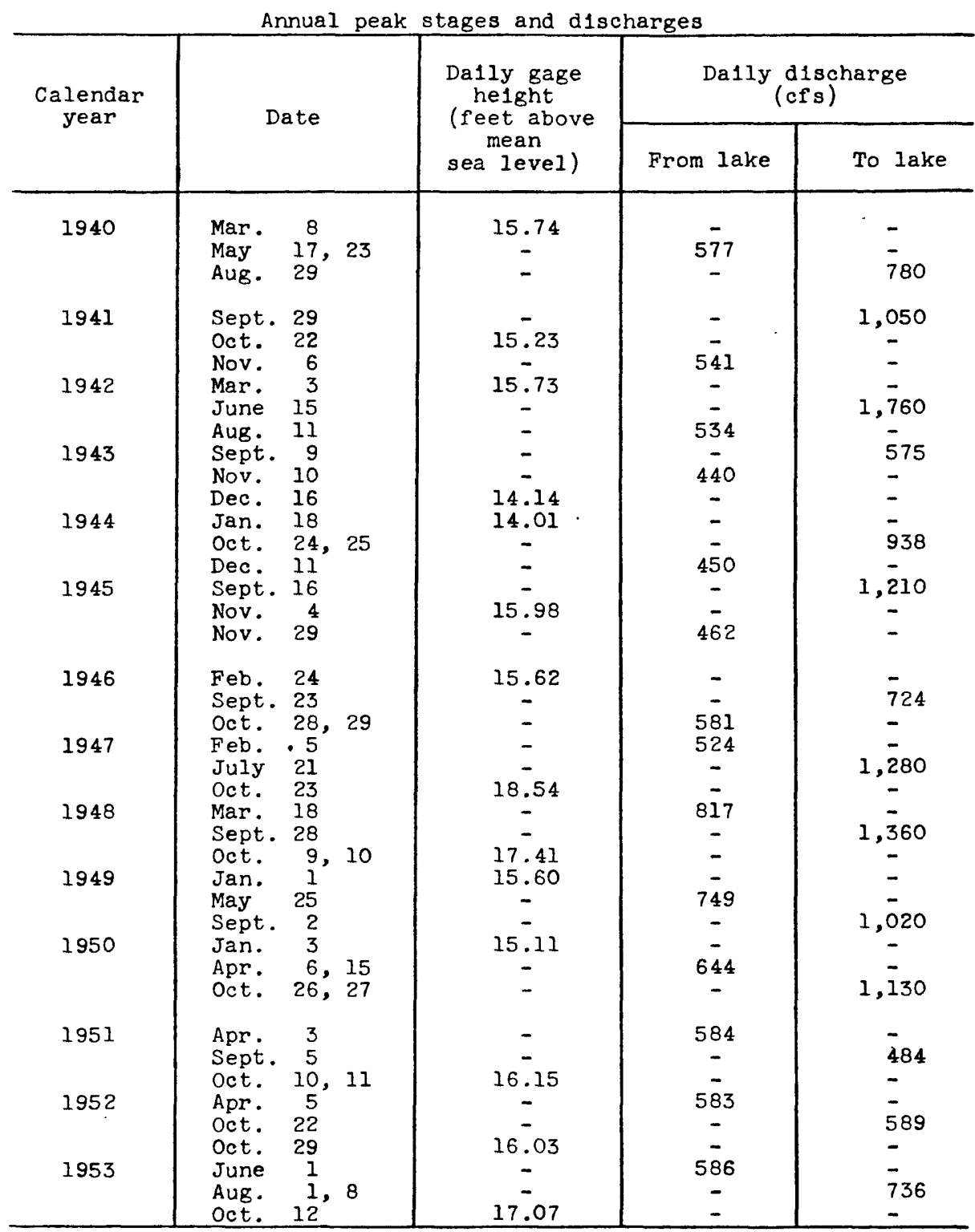




\section{Lake Okeechobee and the Everglades}

(70) West Palm Beach Canal at West Palm Beach, Fla.

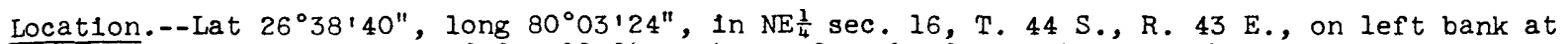
upstream side of lock and dam $20 \mathrm{ft}$ upstream from bridge on State Highway 805 on Polnsettia Avenue and 4.9 miles south of court house in West Palm Beach.

Records avallable.--U. S. Geological Survey: November 1939 to December 1953, da1ly gage he1ghts and discharges.

Gage.--Water-stage recorder. Datum of gage is at mean sea level (State Road Department benchmark). Prior to Apr. 26, 1940, staff gage, and Apr. 26, 1940, to Dec. 19, 1949, waterstage recorder at same site at datum $0.25 \mathrm{ft}$ higher. (All gage heights tabulated below have been reduced to present datum, at mean sea level).

Stage-discharge relation.--Head-discharge relation for flow through 4 bays in spillway dam and stage-discharge relation for flow through lock chamber defined by current-meter measurements.

Historical data.--Maximum stage known, $13.20 \mathrm{ft}$, present datum, oct. 23, 24, 1924, from records by Everglades Drainage District (discharge, 8,570 cfs).

Remarks.--Flow regulated by manipulation of stoplogs in dam and gates in lock chamber for irrigation and drainage purposes by Central and Southern Florida Flood Control District. Lock chamber not used for navigation.

Annual peak stages and discharges

\begin{tabular}{|c|c|c|c|c|c|c|c|}
\hline $\begin{array}{l}\text { Calendar } \\
\text { year }\end{array}$ & Date & $\begin{array}{c}\text { Gage height } \\
\text { (feet above } \\
\text { mean } \\
\text { sea level) }\end{array}$ & $\begin{array}{c}\text { Daily } \\
\text { discharge } \\
\text { (cfs) }\end{array}$ & $\begin{array}{l}\text { Calendar } \\
\text { year }\end{array}$ & Date & $\begin{array}{c}\text { Gage helght } \\
\text { (feet above } \\
\text { mean } \\
\text { sea level) }\end{array}$ & $\begin{array}{l}\text { Daily } \\
\text { discharge } \\
\text { (cfs) }\end{array}$ \\
\hline 1924 & $\begin{array}{l}\text { Oct. } 23 \\
\text { or } 24\end{array}$ & 13.20 & 8,570 & & $\begin{array}{c}\text { May } 14, \text { Nov. } 29 \\
\text { Sept. } 22\end{array}$ & 9.79 & $2, \overline{680}$ \\
\hline 1940 & $\begin{array}{l}\text { Sept. } \\
\text { Oct. } 25\end{array}$ & 9.54 & $\begin{array}{c}3,780 \\
-\end{array}$ & $\begin{array}{l}1947 \\
1948 \\
1949\end{array}$ & $\begin{array}{lr}\text { Oct. } & 13 \\
\text { Oct. } & 7 \\
\text { Dec. } & 26 \\
\text { Dec. } & 31\end{array}$ & $\begin{array}{r}10.89 \\
9.94 \\
9.52\end{array}$ & $\begin{array}{l}5,280 \\
4,760 \\
-780\end{array}$ \\
\hline 1941 & $\begin{array}{ll}\text { June } & 14 \\
\text { Sept. } & 24\end{array}$ & $\begin{array}{c}9.33 \\
-\end{array}$ & $4, \overline{280}$ & 1950 & $\begin{array}{l}\text { Oct. } 19 \\
\text { Nov. } 27\end{array}$ & 9.27 & 4,160 \\
\hline $\begin{array}{l}1942 \\
1943\end{array}$ & $\begin{array}{l}\text { Apr. } 18 \\
\text { Oct. } \quad 3\end{array}$ & $\begin{array}{c}10.33 \\
-\end{array}$ & $\begin{array}{l}5,320 \\
2,060\end{array}$ & 1951 & Oct. 15 & 9.86 & - \\
\hline 1944 & $\begin{array}{ll}\text { Nov. } & 1 \\
\text { Oct. } & 16 \\
\text { Oct. } & 18\end{array}$ & $\begin{array}{l}9.73 \\
9.93 \\
-\end{array}$ & $3, \overline{0} 40$ & 1952 & $\begin{array}{lr}\text { Oct. } & 16 \\
\text { Feb. } & 4 \\
\text { Oct. } & 28\end{array}$ & 9.33 & $\begin{array}{l}4,060 \\
2, \overline{850}\end{array}$ \\
\hline 1945 & $\begin{array}{l}\text { June } 23 \\
\text { Sept. } 18\end{array}$ & $\begin{array}{c}9.95 \\
- \\
\end{array}$ & $3, \overline{120}$ & 1953 & $\begin{array}{lr}\text { June } & 6 \\
\text { Oct. } & 10 \\
\end{array}$ & $\begin{array}{c}9.36 \\
- \\
\end{array}$ & $4, \overline{150}$ \\
\hline
\end{tabular}




\section{Lake Okeechobee and the Everglades}

(71) H1llsboro Canal at Belle Glade, Fla.

Location.--Lat $26^{\circ} 41^{\prime}$, long $80^{\circ} 40^{\prime}$, in sec. 31, T. $43 \mathrm{~S} .$, R. $37 \mathrm{E}$., on southwest bank 20 ft northwest of bridge on State H1ghway 15 in Belle Glade, 3 miles southeast of Lake Okeechobee.

Records ava1lable.--U. S. Geological Survey: January 1940 to October 1942, da1ly gage helghts and discharges measured at sem1-weekly to weekly intervals; October 1942 to September 1950, dally gage helghts and discharges.

Gage.-Water-stage recorder. Datum of gage 1s at mean sea level, datum of 1929. Prior to Nay 11,1940 , staff gage at same site at datum $11.18 \mathrm{ft}$ higher. May 11, 1940, to Mar. 14, 1944, water-stage recorder at same site at datum $1.69 \mathrm{ft}$ lower than present datum. (All gage helghts tabulated below have been reduced to present datum, at mean sea level).

Stage-discharge relation.--There is no definite relation between stage and discharge at this station. Ratings are based on relations between velocity at a point and mean velocity and between stage and area as defined by current-meter measurements. Velocity at a point observed three times a day.

Remarks.--Flow regulated by hurricane gates at Lake Okeechobee. Flow occaslonally reversed after periods of considerable rainfall owing to downstream natural dralnage and to pumping from agricultural lands in Everglades.

Annual peak stages and discharges

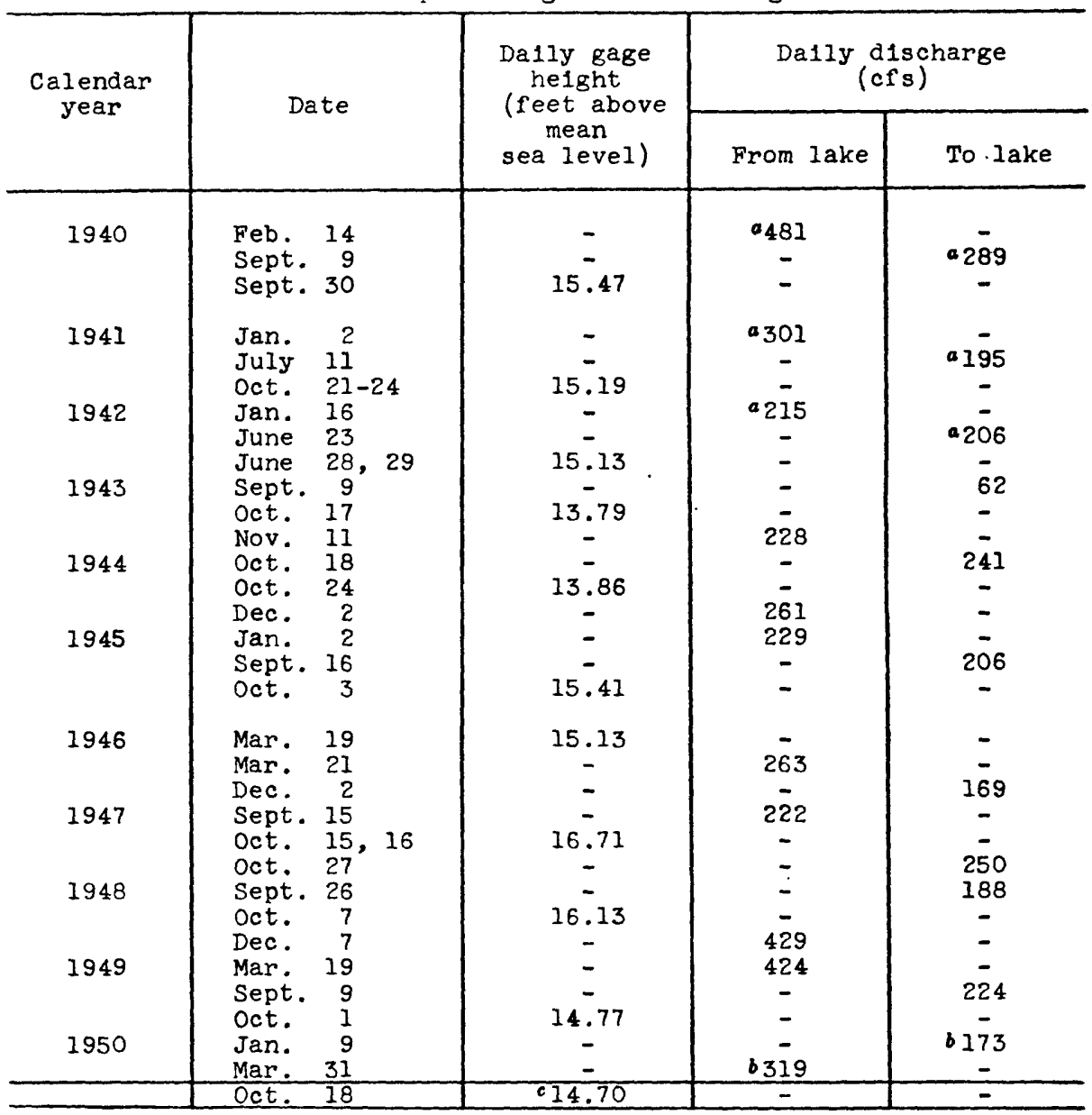

a Maximum measured.

- For period January to September; may have been exceeded during porlod october to December.

c At site 6.0 miles downstream. 


\section{Lake Okeechobee and the Everglades}

(72) Hillsboro Canal at Shawano Plantation, Fla.

Location.--Lat $26^{\circ} 33^{\prime}$, long $80^{\circ} 31^{\prime}$, in sec. 14, T. $45 \mathrm{~S} ., \mathrm{R} .38 \mathrm{E}$, on pier on right bank at offlce of Shawano Plantation at end of State Highway 827,7 miles southeast of junction with U. S. Highway 441 and $13 \frac{1}{2}$ miles southeast of Belle Glade.

Records avallable.--Shawano Plantation, Inc.: Station established at some unknown date prior to January 1929. Prior records were lost in hurricane in 1928. January 1929 to September 1940, da1ly gage heights. U. S. Geological Survey: September 1940 to July 1952, da1ly gage helghts.

Gage.--Staff gage read once dally. Datum of gage is $1.44 \mathrm{ft}$ below mean sea level (levels by Soll Conservation Service, U. S. Department of Agriculture).

Stage-discharge relation.--Not defined.

Annual peak stages

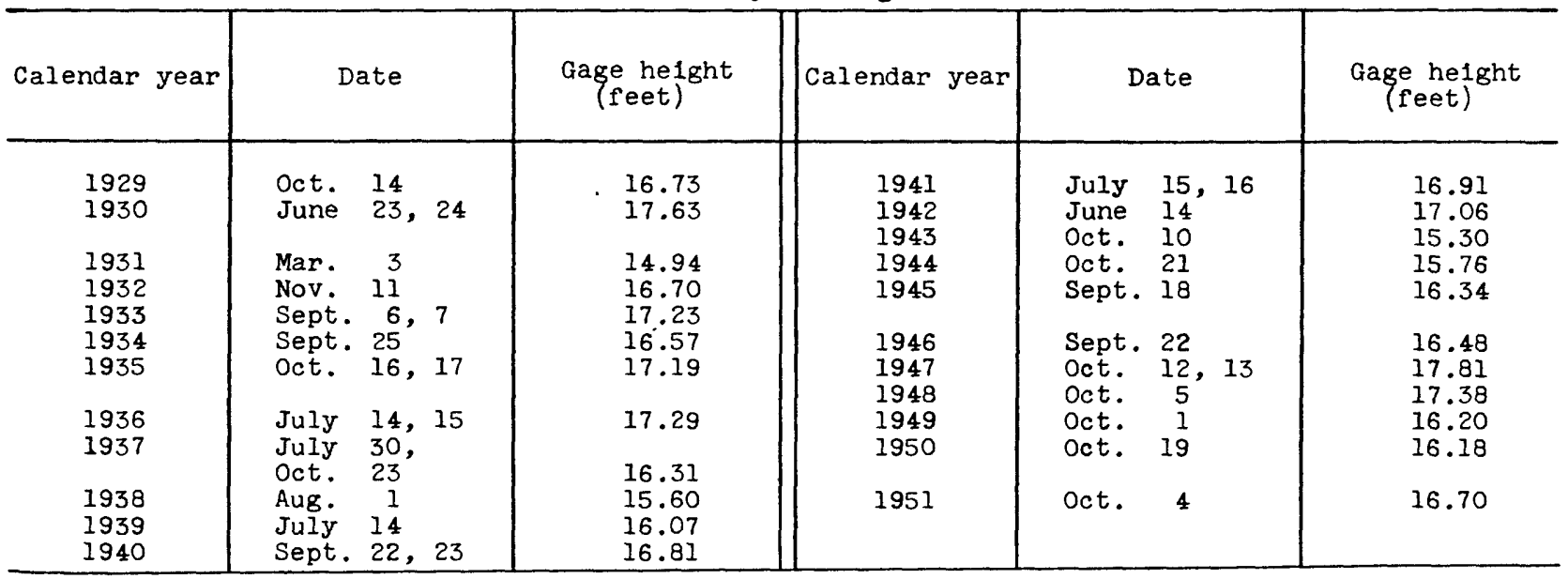




\section{Lake Okeechobee and the Everglades}

(73) H1lisboro Canal near Deerfield Beach, Fla.

Location.--Lat $26^{\circ} 19^{\prime} 39^{\prime \prime}$, long $80^{\circ} 07 ' 52^{\prime \prime}$, in SW $\frac{1}{4}$ sec. 35 , T. 47 S., R. 42 E., at upstream end of lock at right end of dam, $1.8 \mathrm{mlles}$ west of Deerfield Beach, and $4.4 \mathrm{mlles}$ downstream from bridge on State Highway 7 .

Records avallable.--U. S. Geological Survey: November 1939 to December 1953, da1ly gage helghts and discharges.

Gage.--Water-stage recorder. Datum of gage is at mean sea level, datum of 1929 (Corps of Engineers benchmark). Prior to Apr. 15, 1940, staff gage at same s1te at datum $0.92 \mathrm{ft}$ lower. Since July 3i, 1947, aux1liary water-stage recorder at downstream end of lock or at site $500 \mathrm{ft}$ downstream from lock.

Stage-discharge relation.--Head-discharge relation for flow through 5 bays in splliway dam defined by current-meter measurements.

Remarks.--Flow partly regulated at station by Central and Southern Florida Flood Control District for irrigation and drainage and by a flood-control levee 11 miles above station. Pumps above station divert water for irrigation during growing season.

Annual peak stages and discharges

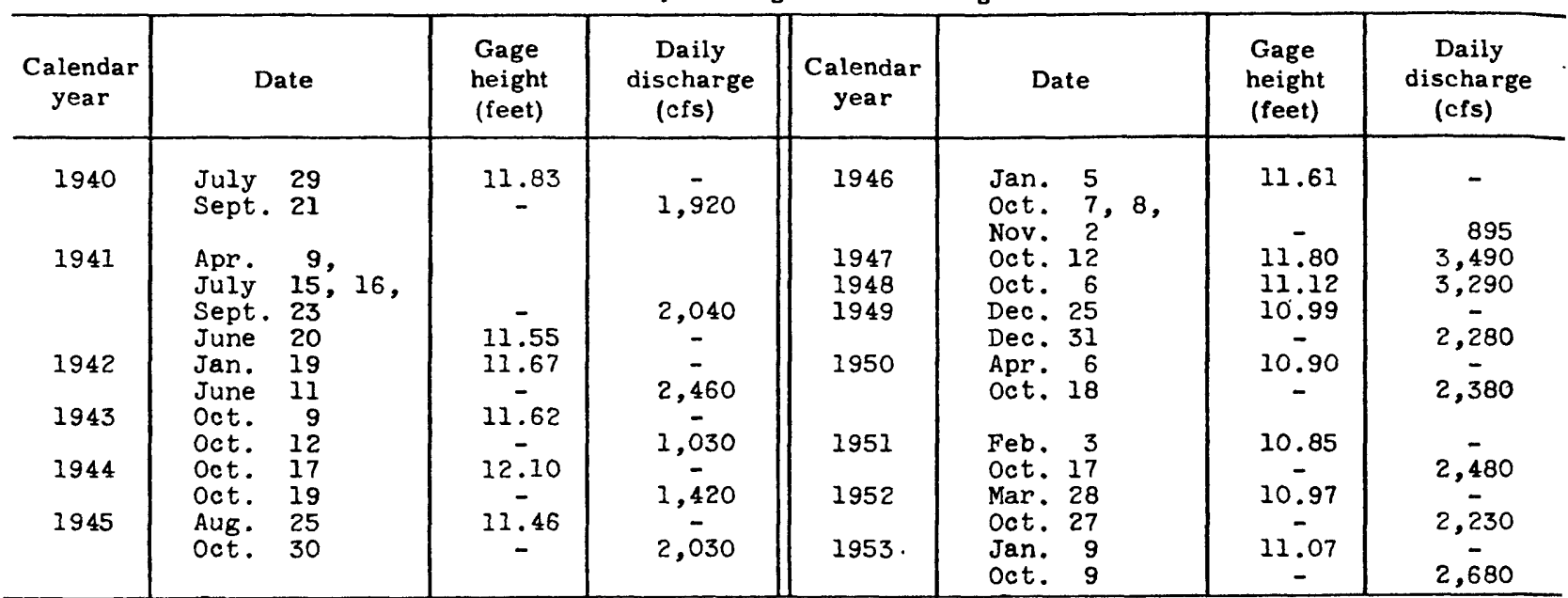


Lake Okeechobee and the Everglades

(74) North New River Canal at South Bay, Fla.

Location. - Lat $26^{\circ} 40^{\prime}$, long $80^{\circ} 43^{\prime}$, in sec. 14, T. 44 S., R. 36 E., on right bank on downstream side of lock and dam in South Bay, $410 \mathrm{ft}$ downstream from bridge on State H1ghway 80 , and $2.5 \mathrm{mlles}$ south of Iake Okeechobee.

Records ava1lable.--U. S. Geological Survey: November 1939 to March 1942, da1ly gage helghts and weekly or twice weekly discharge measurements. Apr 111942 to December 1953 , da1ly gage helghts and discharges.

Gage.--Staff gage read twice dally. Datum of gage is at mean sea level, datum of 1929 .

Stage-discharge relation.--There is no definlte relation between stage and discharge at this station. Ratings are based on relations between velocity at a point and mean veloclty and between stage and area as defined by current-meter measurements. Velocity at a point observed twlce dally.

H1storlcal data.-Flood of July 27, 28, 1926, reached a stage of $20.56 \mathrm{ft}$, from records by Everglades Drainage District.

Remarks.--Flow regulated by hurricane gates at Lake Okeechobee and by operation of drainage pumps.

Annual peak stages and discharges

\begin{tabular}{|c|c|c|c|c|c|}
\hline \multirow{2}{*}{$\begin{array}{l}\text { Calendar } \\
\text { year }\end{array}$} & \multirow{2}{*}{\multicolumn{2}{|c|}{ Date }} & \multirow{2}{*}{$\begin{array}{c}\text { Dally gage } \\
\text { helght } \\
\text { (feet above } \\
\text { mean } \\
\text { sea level) }\end{array}$} & \multicolumn{2}{|c|}{$\begin{array}{l}\text { Da1ly discharge } \\
\text { (cfs) }\end{array}$} \\
\hline & & & & From lake & To lake \\
\hline 1926 & July & 27,28 & 20.56 & - & - \\
\hline 1940 & $\begin{array}{l}\text { May } \\
\text { Sept. } \\
\text { Oct. }\end{array}$ & $\begin{array}{l}23 \\
24,28,29 \\
18\end{array}$ & 14.78 & $\begin{array}{c}a 341 \\
- \\
-\end{array}$ & $\overline{-}$ \\
\hline 1941 & $\begin{array}{l}\text { May } \\
\text { July } \\
\text { July }\end{array}$ & $\begin{array}{l}12 \\
20 \\
25\end{array}$ & 14.97 & $\begin{array}{c}a 21 \\
- \\
-\end{array}$ & $\overline{-}$ \\
\hline 1942 & $\begin{array}{l}\text { June } \\
\text { June } \\
\text { Nov. }\end{array}$ & $\begin{array}{l}10 \\
14 \\
26\end{array}$ & 14.62 & $\begin{array}{l}- \\
254\end{array}$ & $\begin{array}{c}404 \\
- \\
-\end{array}$ \\
\hline 1943 & $\begin{array}{l}\text { Nov. } \\
\text { Nov. }\end{array}$ & $\begin{array}{l}11 \\
17-19\end{array}$ & 12.55 & 312 & $\overline{-}$ \\
\hline 1944 & $\begin{array}{l}\text { Mar. } \\
\text { Mar. } \\
\text { Oct. }\end{array}$ & $\begin{array}{r}9 \\
1 \\
19\end{array}$ & 13.17 & $\begin{array}{l}325 \\
- \\
-\end{array}$ & $\overline{27}$ \\
\hline 1945 & $\begin{array}{l}\text { Jan. } \\
\text { Oct. }\end{array}$ & $\begin{array}{r}2 \\
17\end{array}$ & 14.14 & 241 & - \\
\hline 1946 & $\begin{array}{l}\text { Sept. } \\
\text { Dec. }\end{array}$ & $\begin{array}{l}22 \\
15\end{array}$ & 13.96 & $\overline{365}$ & - \\
\hline 1947 & $\begin{array}{l}\text { Sept. } \\
\text { oct. }\end{array}$ & $\begin{array}{l}30 \\
15,16\end{array}$ & 16.38 & & - \\
\hline 1948 & $\begin{array}{l}\text { May } \\
\text { Oct. } \\
\text { Nov. }\end{array}$ & $\begin{array}{l}19 \\
11 \\
22\end{array}$ & $\begin{array}{c}- \\
15.73 \\
-\end{array}$ & $\begin{array}{l}506 \\
- \\
-\end{array}$ & $\frac{-}{43}$ \\
\hline 1949 & $\begin{array}{l}\text { Mar. } \\
\text { Oct. }\end{array}$ & 11 & $14 \overline{4}$ & $\begin{array}{l}742 \\
-\end{array}$ & - \\
\hline 1950 & $\begin{array}{l}\text { Jan. } \\
\text { Apr. } \\
\text { Oct. }\end{array}$ & $\begin{array}{r}1 \\
7 \\
18\end{array}$ & $\begin{array}{c}13.89 \\
-\end{array}$ & $\overline{602}$ & $\frac{-}{379}$ \\
\hline 1951 & $\begin{array}{l}\text { Oct. } \\
\text { Nov. } \\
\text { Dec. }\end{array}$ & $\begin{array}{l}10 \\
26,27 \\
15\end{array}$ & $\begin{array}{c}15.20 \\
-\end{array}$ & $\begin{array}{l}\overline{544} \\
\overline{5}\end{array}$ & $\begin{array}{l}58 \\
-\end{array}$ \\
\hline 1952 & $\begin{array}{l}\text { Jan. } \\
\text { Oct. } \\
\text { Nov. }\end{array}$ & $\begin{array}{r}7 \\
21 \\
30\end{array}$ & 15.35 & $\begin{array}{l}677 \\
- \\
-\end{array}$ & $\overline{-}$ \\
\hline 1953 & $\begin{array}{l}\text { Apr. } \\
\text { Oct: } \\
\text { Dec. }\end{array}$ & $\begin{array}{l}20 \\
10 \\
13 \\
\end{array}$ & $\begin{array}{c}- \\
15.88 \\
- \\
\end{array}$ & $\begin{array}{l}642 \\
- \\
- \\
\end{array}$ & $\overline{115}$ \\
\hline
\end{tabular}

a Result of discharge measurement. 


\section{Lake Okeechobee and the Everglades}

(75) North New River Canal at 26-mile bend, near Fort Lauderdale, Fla.

Location.--Lat $26^{\circ} 13^{\prime} 50^{\prime \prime}$, long $80^{\circ} 27^{\prime} 35^{\prime \prime}$, in $\mathrm{NE}_{\frac{1}{4}} \mathrm{sec} .32, \mathrm{~T} .48 \mathrm{~S} ., \mathrm{R} .39 \mathrm{E}$, on right bank $50 \mathrm{ft}$ upstream from control dam at $26-\mathrm{mlle}$ bend on $U$. S. H1ghway 27,22 miles northwest of Fort Lauderdale.

Records ava1lable.--U. S. Geological Survey: June 1942 to December 1952, da1ly gage he1ghts and perlodic discharge measurements.

Gage.--Water-stage recorder. Datum of gage is at mean sea level (State Road Department benchmark).

Stage-discharge relation.--Not defined; stage is affected by manipulation of stoplogs in the control.

Remarks.--Flow is regulated by hurricane gates at Lake Okeechobee and manipulation of stoplogs in the control.

Annual peak stages

\begin{tabular}{|c|c|c|c|c|c|}
\hline Calendar year & Date & $\begin{array}{l}\text { Gage helght } \\
\text { feet) }\end{array}$ & Calendar year & Date & $\begin{array}{l}\text { Gage helght } \\
\text { feet) }\end{array}$ \\
\hline $\begin{array}{l}1943 \\
1944 \\
1945\end{array}$ & $\begin{array}{lr}\text { Nov. } & 19 \\
\text { Aug. } & 4 \\
\text { Jan. } & 10\end{array}$ & $\begin{array}{l}10.62 \\
11.05 \\
10.51\end{array}$ & $\begin{array}{l}1948 \\
1949 \\
1950\end{array}$ & $\begin{array}{lr}\text { Oct. } & 5 \\
\text { Mar. } & 11 \\
\text { Oct. } 20\end{array}$ & $\begin{array}{l}11.82 \\
10.29 \\
10.60\end{array}$ \\
\hline $\begin{array}{l}1946 \\
1947 \\
\end{array}$ & $\begin{array}{l}\text { Aug. } 24 \\
\text { oct. } 12\end{array}$ & $\begin{array}{l}10.80 \\
12.97\end{array}$ & $\begin{array}{l}1951 \\
1952 \\
\end{array}$ & $\begin{array}{ll}\text { Oct. } & 4 \\
\text { Nov. } & 6 \\
\end{array}$ & $\begin{array}{l}10.60 \\
10.74\end{array}$ \\
\hline
\end{tabular}


Lake Okeechobee and the Everglades

(76) North New River Canal near Fort Lauderdale, Fla.

Location.--Lat $26^{\circ} 05^{\prime} 39^{\prime \prime}$, long $80^{\circ} 13^{\prime} 48^{\prime \prime}$, in SW $\frac{1}{4}$ sec. 14, T. 50 S., R. 41 E., on right bank $20 \mathrm{ft}$ upstream from lock and dam on State Highway 84,6 miles southwest of Fort'Lauderdale.

Records avallable.--U. S. Geological Survey: November 1939 to December 1953, da1ly gage helghts and discharges.

Gage.--Water-stage recorder. Datum of gage is at mean sea level, datum of 1929 (Corps of Engineers benchmark). Prior to Apr. 13, 1940, staff gage at same site and datum. Auxillary water-stage recorder at downstream end of lock chamber. Aug. 1, 1947, to July 20, 1950, at site 500 ft downstream.

Stage-discharge relation.--Head-discharge relation for flow through 8 bays in spillway dam defined by current-meter measurements.

H1storical data.--Maximum discharge known, 5,400 cfs 0ct. 15, 1929 (gage height, 7.66 ft, present datum, from records by Everglades Drainage District.

Remarks.--Flow regulated at and above station by dams for irrigation, drainage, and flood and fire control.

Annual peak stages and discharges

\begin{tabular}{|c|c|c|c|c|c|c|c|}
\hline $\begin{array}{c}\text { Calendar } \\
\text { year }\end{array}$ & Date & $\begin{array}{c}\text { Gage } \\
\text { height } \\
\text { (feet) }\end{array}$ & $\begin{array}{l}\text { Daily } \\
\text { discharge } \\
\text { (cfs) }\end{array}$ & $\begin{array}{c}\text { Calendar } \\
\text { year }\end{array}$ & Date & $\begin{array}{c}\text { Gage } \\
\text { height } \\
\text { (feet) }\end{array}$ & $\begin{array}{c}\text { Daily } \\
\text { discharge } \\
\text { (cfs) }\end{array}$ \\
\hline 1929 & oct. 15 & 7.66 & $a_{5}, 400$ & 1947 & Oct. 17 & 10.83 & - \\
\hline 1940 & $\begin{array}{l}\text { Sept. } 12,13, \\
\text { Oct. } 11 \\
\text { Oct. } 24\end{array}$ & 6.81 & 1,440 & $\begin{array}{l}1948 \\
1949\end{array}$ & $\begin{array}{lr}\text { Oct. } & 5 \\
\text { Oct. } & 9 \\
\text { Mar. } & 7 \\
\text { Oct. } & 1\end{array}$ & $\begin{array}{l}- \\
9.39 \\
6.46 \\
-\end{array}$ & $\begin{array}{c}2,800 \\
- \\
- \\
2,110\end{array}$ \\
\hline 1941 & $\begin{array}{lr}\text { June } & 20 \\
\text { Aug. } & 8\end{array}$ & 6.85 & $1, \overline{970}$ & 1950 & $\begin{array}{l}\text { Oct. } 19 \\
\text { Nov. } 27,28\end{array}$ & 6.14 & 1,690 \\
\hline 1942 & $\begin{array}{l}\text { Mar. } \\
\text { June } \\
12\end{array}$ & 6.18 & $1, \overline{8} 20$ & 1951 & $\operatorname{Jan} .5,7$ & 6.38 & - \\
\hline 1943 & $\begin{array}{ll}\text { July } 10 \\
\text { Sept. } 13-15\end{array}$ & $\begin{array}{c}6.87 \\
-\end{array}$ & 608 & 1952 & $\begin{array}{l}\text { Oct. } 23 \\
\text { Jan. } 29\end{array}$ & 6.35 & 1,460 \\
\hline 1944 & $\begin{array}{l}\text { Aug. } \quad 2 \\
\text { Oct. } 23\end{array}$ & $\begin{array}{c}6.78 \\
-\end{array}$ & $1 . \overline{0} 70$ & & $\begin{array}{l}\text { Oct. } 29, \\
\text { Nov. } 3\end{array}$ & - & 1,860 \\
\hline 1945 & $\begin{array}{l}\text { Oct. } 1 \\
\text { Nov. } 13-15\end{array}$ & $\begin{array}{c}6.77 \\
-\end{array}$ & $1, \overline{3} 90$ & 1953 & $\begin{array}{l}\text { Mar. } 17 \\
\text { Oct. } 20\end{array}$ & $\begin{array}{c}5.86 \\
-\end{array}$ & $1, \overline{700}$ \\
\hline 1946 & $\begin{array}{ll}\text { Sept. } & 19 \\
\text { Dec. } & 16\end{array}$ & 6.93 & 1,070 & & & & \\
\hline
\end{tabular}

\footnotetext{
a Peak discharge.
} 
Lake Okeechobee and the Everglades

(77) Miami Canal at Pennsuco, near Mlam1, Fla.

Location.--Lat $25^{\circ} 55^{\prime}$, long $80^{\circ} 22^{\prime}$, in sec. 30, T. 52 S., R. 40 E., on left bank at upstream slde of road bridge in Pennsuco, $7.4 \mathrm{miles}$ northwest of Miami water plant in H1aleah and 14.3 miles northwest of Miam1 Post office.

Records ava1lable.--Grahams Dalry: July 1926 to November 1939, da1ly gage he1ghts. U. S. Geolog1cal Survey: November 1939 to December 1953, da1ly gage helghts. November 1939 to July 1943, da1ly discharges.

Gage.--Water-stage recorder. Datum of gage 1s at mean sea level, datum of 1929. Prior to Sept. 28, 1940, staff gage. Nov. 12, 1939, to Mar. 25, 1953, datum was from 0.54 to $0.60 \mathrm{ft}$ lower. Gage read by employees of Grahams Dalry was approximately at same datum as Geological Survey gage set on Nov. 3, 1939. (All gage heights shown below have been reduced to present datum, at mean sea level).

Stage-d1scharge relation.--Not defined; affected by t1de. D1scharge record for period November 1939 to July 1943 based on current-meter measurements made at approximately weekly intervals.

Annual peak stages and discharges

\begin{tabular}{|c|c|c|c|c|c|c|c|}
\hline $\begin{array}{c}\text { Calendar } \\
\text { year }\end{array}$ & Date & $\begin{array}{c}\text { Gage height } \\
\text { (feet above } \\
\text { mean } \\
\text { sea level) }\end{array}$ & $\begin{array}{c}\text { Da1ly } \\
\text { d1scharge } \\
\text { (cfs) }\end{array}$ & $\begin{array}{c}\text { Calendar } \\
\text { year }\end{array}$ & Date & $\begin{array}{c}\text { Gage helght } \\
\text { (feet above } \\
\text { mean } \\
\text { sea level) }\end{array}$ & $\begin{array}{c}\text { Da1ly } \\
\text { discharge } \\
\text { (cfs) }\end{array}$ \\
\hline $\begin{array}{l}1926 \\
1927 \\
1928 \\
1929 \\
1930 \\
1931 \\
1932 \\
1933 \\
1934 \\
1935 \\
1936 \\
1937 \\
1938 \\
1939 \\
1940\end{array}$ & $\begin{array}{lc}\text { Oct. } & 25 \\
\text { Oct. } & 25 \\
\text { Sept. } & 27 \\
\text { Oct. } & 20 \\
\text { Jan. } & 1 \\
& \\
\text { Sept. } & 28-30 \\
\text { Nov. } & 9 \\
\text { Oct. } & 27 \\
\text { May } & 22,23 \\
\text { Nov. } & 8 \\
& \\
\text { July } & 14 \\
\text { Oct. } & 18 \\
\text { July } & 16 \\
\text { Nov. } & 4 \\
\text { Nov. } & 25-28 \\
\text { Sept. } & 22,23 \\
\text { Nov. } & 25\end{array}$ & $\begin{array}{l}a 7.50 \\
a_{4} .5 \\
a 6.16 \\
a 8.54 \\
a 5.91 \\
a 5.44 \\
a 6.06 \\
a 6.16 \\
a 5.15 \\
a 5.74 \\
a 6.87 \\
a 5.17 \\
a 3.93 \\
a 5.35 \\
- \\
5.89 \\
-\end{array}$ & $\begin{array}{l}- \\
- \\
= \\
- \\
- \\
- \\
- \\
- \\
- \\
- \\
\overline{-} \\
730 \\
\overline{956}\end{array}$ & $\begin{array}{l}1941 \\
1942 \\
1943 \\
1944 \\
1945 \\
1946 \\
1947 \\
1948 \\
1949 \\
1950 \\
1951 \\
1952 \\
1953\end{array}$ & $\begin{array}{l}\text { Feb. } 13,14 \\
\text { Oct. } 6 \\
\text { July } 4 \\
\text { Oct. } 5 \\
\text { Oct. 12, 13 } \\
\text { Oct. } 25,26,27 \\
\text { Nov. } 18 \\
\text { Oct. } 7 \\
\text { Oct. } 12 \\
\text { Oct. } 12 \\
\text { Oct. } 13 \\
\text { Oct. } 18 \\
\text { Oct. } 18 \\
\text { Oct. } 28 \\
\text { Oct. } 9\end{array}$ & $\begin{array}{l}- \\
5.10 \\
5.89 \\
-. \\
5.64 \\
5.14 \\
5.17 \\
4.88 \\
9.00 \\
6.83 \\
5.52 \\
4.35 \\
4.31 \\
5.76 \\
5.93\end{array}$ & $\begin{array}{c}848 \\
- \\
\overline{774} \\
- \\
- \\
- \\
- \\
- \\
- \\
- \\
- \\
-\end{array}$ \\
\hline
\end{tabular}

a Mean da1ly. 
Lake Okeechobee and the Everglades

(78) Miami Canal at water plant, Hialeah, Fla.

Location.--Lat $25^{\circ} 49^{\prime} 38^{\prime \prime}$, long $80^{\circ} 17^{\prime} 15^{\prime \prime}$, In SW $\frac{1}{4}$ sec. 18, T. 53 S., R. 41 E., on left bank at Mlami water plant in Hialeah, on U. S. Highway 27 , haif a mile upstream from 54 th Street bridge.

Records available.--U. S. Geological Survey: January 1940 to December 1953, da1ly gage heights and discharges.

Gage.--Water-stage recorder. Datum of gage $1 \mathrm{~s} 0.01 \mathrm{ft}$ below mean sea level, datum of 1929 (levels by Dade County). Nov. 8, 1940, to Sept. 30, 1946, water-stage recorder on B1scayne Bay at Coconut Grove was used as auxiliary gage for this station and since oct. 1, 1946, at site 2 miles downstream from base gage.

Stage-discharge relation.--Deflned by current-meter measurements; affected by backwater from tides. Discharge computed by using gage helghts and tide ranges at auxiliary gage as a factor.

Remarks.--Some seepage losses above station into c1ty of Mlaml well field for recharge of ground-water withdrawals.

Annual peak stages and discharges

\begin{tabular}{|c|c|c|c|c|c|c|c|}
\hline $\begin{array}{l}\text { Calendar } \\
\text { year }\end{array}$ & Date & $\begin{array}{l}\text { Gage } \\
\text { height } \\
\text { (feet) }\end{array}$ & $\begin{array}{l}\text { Daily } \\
\text { discharge } \\
\text { (cfs) }\end{array}$ & $\begin{array}{l}\text { Calendar } \\
\text { year }\end{array}$ & $\therefore \quad$ Date & $\begin{array}{c}\text { Gage } \\
\text { height } \\
\text { (feet) }\end{array}$ & $\begin{array}{l}\text { Daily } \\
\text { discharge } \\
\text { (cfs) }\end{array}$ \\
\hline 1940 & $\begin{array}{ll}\text { Oct. } & 1 \\
\text { Nov. } & 8-10\end{array}$ & 3.89 & $1, \overline{6} 70$ & $\begin{array}{l}1947 \\
1948\end{array}$ & $\begin{array}{l}\text { Oct. } 15 \\
\text { Oct. } 5 \\
\text { Oct. } 12\end{array}$ & $\begin{array}{c}7.34 \\
5.20 \\
-\end{array}$ & $\begin{array}{c}4,170 \\
2 ; 530\end{array}$ \\
\hline 1941 & $\begin{array}{l}\text { Feb. } \\
\text { Oct. }\end{array}$ & $\overline{3.43}$ & 1,330 & 1949 & & 3.91 & 1,480 \\
\hline 1942 & $\begin{array}{l}\text { Sept. } 3,5 \\
\text { Sept. } 4, \\
\text { Oct. } 12\end{array}$ & $\begin{array}{l}- \\
3.18\end{array}$ & $\begin{array}{c}1,610 \\
-\end{array}$ & 1950 & $\begin{array}{l}\text { Oct. } 18 \\
\text { Nov. } 24,25\end{array}$ & 4.17 & $\overline{982}$ \\
\hline 1943 & $\begin{array}{l}\text { Oct. } 14 \\
\text { Nov. } 30\end{array}$ & 3.26 & $\overline{823}$ & 1951 & $\begin{array}{ll}\text { Oct. } & 2 \\
\text { Dec. } & 5\end{array}$ & 3.25 & $\overline{878}$ \\
\hline 1944 & $\begin{array}{ll}\text { Oct. } & 18 \\
\text { Nov. } & 20-24\end{array}$ & 3.59 & $\overline{684}$ & 1952 & $\begin{array}{l}\text { Oct. } 27 \\
\text { Oct. } 28\end{array}$ & 4.23 & $1, \overline{5} 60$ \\
\hline 1945 & $\begin{array}{l}\text { Sept. } 15 \\
\text { Nov. } 19-22\end{array}$ & 4.42 & $1, \overline{540}$ & 1953 & $\begin{array}{ll}\text { Oct. } & 9 \\
\text { Oct. } & 9,10\end{array}$ & 4.11 & $1, \overline{6} 00$ \\
\hline 1946 & $\begin{array}{ll}\text { Oct. } & 8,9,13 \\
\text { Oct. } & 7\end{array}$ & 3.40 & $\begin{array}{c}1,250 \\
- \\
\end{array}$ & & & & \\
\hline
\end{tabular}

(79) Tamlam1 Canal outlets, Miam1 to Monroe, Fla.

Location.--Lat $25^{\circ} 45^{\prime} 40^{\prime \prime}$, long $80^{\circ} 49^{\prime} 40^{\prime \prime}$, in NE $\frac{1}{4}$ seo. 21 , T. 54 S., R. 35 E., at 40-m1le bend on U. S. Highway 41,38 miles west of Miami.

Records available.--U. S. Geological Survey: November 1939 to December 1953, da1ly gage heights and discharges.

Gage.--Water-stage recorder. Datum of gage is at mean sea level, datum of 1929. Dec. 9, 1939, to Aug. 30,1949 , staff gage read once daily at same site at datum 0.87 ft lower. Aug. 31, 1949, to Dec. 28, 1951, staff gage at present site and datum. (Al1 gage helghts tabulated below have been reduced to present datum, at mean sea level).

Stage-discharge relation.--Prior to oct. 1, 1949, no stage-d1scharge relation was developed and dally discharges were estimated on basis of gage helghts and interpolation between ourrentmeter measurements. Since Oct. 1 , 1949, daliy discharges computed from very unstable stagedischarge realtions. Considerable shifting occurs at all stages.

Remarks.--Discharge consists of runoff from Everglades as represented by flow through all outlets of Tamiaml Canal from Monroe, 55 miles west of Miaml, to point 18 miles west of Mlaml where a levee aids in diverting flow through 60 outlets to the area immediately south of canal. 
Lake Okeechobee and the Everglades

(79) Tamlam1 Canal outlets, Miam1 to Monroe, Fla.--Cont1nued

Annual peak stages and discharges

\begin{tabular}{|c|c|c|c|c|c|c|c|}
\hline $\begin{array}{c}\text { Calendar } \\
\text { year }\end{array}$ & Date & $\begin{array}{l}\text { 40-mile bend } \\
\text { gage helght } \\
\text { feet) }\end{array}$ & $\begin{array}{c}\text { Da1ly } \\
\text { d1scharge } \\
\text { (cfs) }\end{array}$ & $\begin{array}{c}\text { Calendar } \\
\text { year }\end{array}$ & Date & $\begin{array}{l}\text { 40-mile bend } \\
\text { gage height } \\
\text { feet) }\end{array}$ & $\begin{array}{c}\text { Dally } \\
\text { discharge } \\
\text { (cfs) }\end{array}$ \\
\hline $\begin{array}{l}1941 \\
1942\end{array}$ & $\begin{array}{l}\text { Sept. } 11,12 \\
\text { Sept. } 13 \\
\text { Feb. } 11 \\
\text { July } 17 \\
\text { June } 11-13\end{array}$ & $\begin{array}{l}- \\
8.75 \\
- \\
8.92 \\
8.89\end{array}$ & $\begin{array}{c}1,670 \\
- \\
1,430 \\
- \\
-\end{array}$ & $\begin{array}{l}1946 \\
1947 \\
1948 \\
1949 \\
1950\end{array}$ & $\begin{array}{lr}\text { Aug. } & 23 \\
\text { Sept. } & 25 \\
\text { Oct. } & 12 \\
\text { Oct. } & 6 \\
\text { Sept. } & 30 \\
\text { Oct. } & 15\end{array}$ & $\begin{array}{c}8.47 \\
- \\
10.3 \\
10.03 \\
9.06 \\
8.92\end{array}$ & $\begin{array}{r}- \\
1,160 \\
17,000 \\
12,900 \\
3,500 \\
2,610\end{array}$ \\
\hline $\begin{array}{l}1943 \\
1944\end{array}$ & $\begin{array}{l}\text { Sept. } 14-16 \\
\text { Sept. } 15 \\
\text { Aug. } 7, \\
\text { oct. } 27,28 \\
\text { Oct. } 22 \\
\text { Sept. } 18 \\
\text { Sept. } 22\end{array}$ & $\begin{array}{c}8.93 \\
- \\
8.43 \\
- \\
- \\
8.95\end{array}$ & $\begin{array}{c}1, \overline{820} \\
\overline{5} \\
2,140 \\
-\end{array}$ & $\begin{array}{l}1951 \\
1952 \\
1953\end{array}$ & $\begin{array}{ll}\text { Oct. } & 10,11 \\
\text { Oct. } & 21 \\
\text { Oct. } & 25 \\
\text { Oct. } & 8 \\
\text { Nov. } & 1,2\end{array}$ & $\begin{array}{c}8.44 \\
8.97 \\
- \\
9.04 \\
-\end{array}$ & $\begin{array}{l}1,210 \\
- \\
2,720 \\
- \\
4,450\end{array}$ \\
\hline
\end{tabular}

a Aoproximate; water over gage.

(80) Lake Trafford near Immokalee, Fla.

Location.--Lat $26^{\circ} 26^{\prime}$, Iong $81^{\circ} 29^{\prime}$, In NWt sec. 35 , T. 46 S., R. 28 E., on north shore of 1 ake, $10 \mathrm{ft}$ lakeward from dock at Pepper's Camp, $4 \frac{1}{2} \mathrm{mll}$ es west of Immokalee.

Records available.--U. S. Geolog1cal Survey: March 1941 to December 1953, da1ly gage he1ghts.

Gage.--Staff gage read once da1ly. Datum of gage is $16.00 \mathrm{ft}$ ( $\pm 1.00 \mathrm{ft}$ ), above mean sea level

(State Road Department benchmark which was reputed to be in error by one foot). Prior to

Aug. 21, 1952, at various sites in same vicinity at same datum.

Annual peak stages

\begin{tabular}{|c|c|c|c|c|c|}
\hline Calendar year & Date & $\begin{array}{l}\text { Gage helght } \\
\text { feet) }\end{array}$ & Calendar year & Date & $\begin{array}{l}\text { Gage helght } \\
\text { feet) }\end{array}$ \\
\hline $\begin{array}{l}1941 \\
1942 \\
1943 \\
1944 \\
1945 \\
1946 \\
1947 \\
\end{array}$ & $\begin{array}{ll}\text { July } & 17 \\
\text { June } & 17-20,25,26 \\
\text { bet. } 3 \\
\text { Sept. } 13 \\
\text { Sept. } 19 \\
\text { Sept. } 20 \\
\text { Sept. } 18 \\
\end{array}$ & $\begin{array}{l}5.8 \\
4.46 \\
4.12 \\
3.78 \\
5.00 \\
4.66 \\
6.60\end{array}$ & $\begin{array}{l}1948 \\
1949 \\
1950 \\
1951 \\
1952 \\
1953 \\
\end{array}$ & $\begin{array}{l}\text { Sept. } 27-29 \text {, } \\
\text { Oct. } \quad 2 \\
\text { Oct. } 4 \\
\text { Jan. } 1,2 \\
\text { Oct. } \quad 3 \\
\text { Oct. } 24,25 \\
\text { Oct. } 11\end{array}$ & $\begin{array}{l}4.70 \\
4.82 \\
3.34 \\
5.82 \\
4.60 \\
5.12\end{array}$ \\
\hline
\end{tabular}


Lake Okeechobee and the Everglades

(81) Imperial River near Bonita Springs, Fla.

,

Location.--Lat $26^{\circ} 20^{\prime}$, long $81^{\circ} 45^{\prime}$, in sec. 36, T. $47 \mathrm{~S}$, R. $25 \mathrm{E}$, on right bank $1 \frac{1}{2}$ miles east of Bonita Springs.

Records avallable.--U. S. Geological Survey: May 1940 to December 1953, da1ly gage heights and discharges.

Gage.--Water-stage recorder. Datum of gage is at mean sea level, datum of 1929 . Pr1or to Sept. 10, 1941, staff gage at same site and datum.

Stage-discharge relation.--Defined by current-meter measurements. Slight tide effect at low stages.

H1storical data.--Flood of June 15, 1936, reached a stage of $13.4 \mathrm{ft}$, from floodmarks.

Annual peak stages and discharges

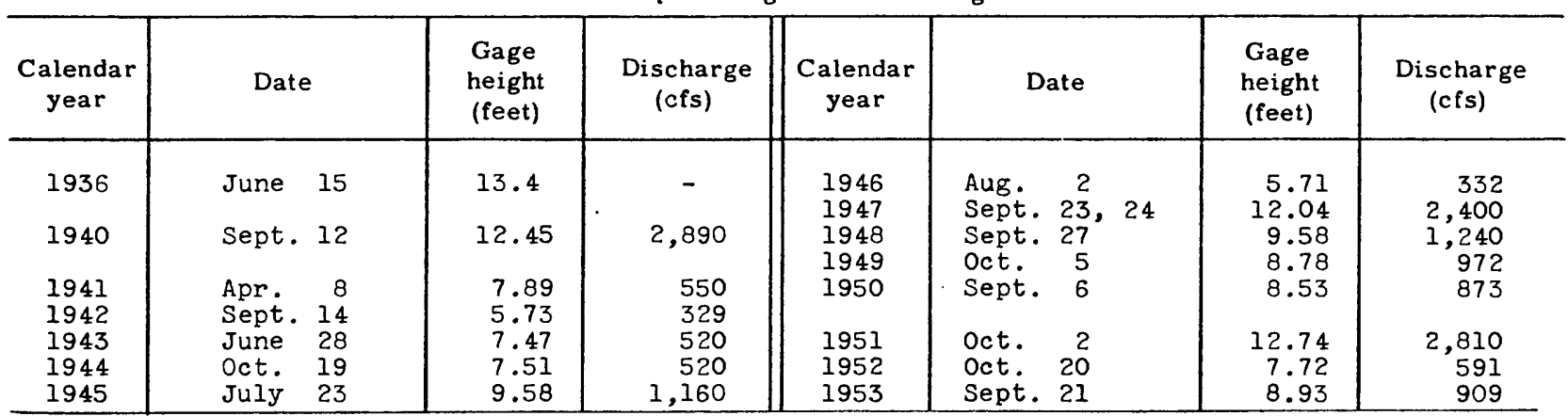

(82) Caloosahatchee Canal at Moore Haven, Fla.

Location.--Lat $26^{\circ} 50^{\prime}$, long $81^{\circ} 05^{\prime}$, in sec. 12, T. 42 S., R. 32 E., on right bank at Moore Haven, $0.5 \mathrm{~m} 11 \mathrm{e}$ downstream from hurricane gate and lock 1 at Lake Okeechobee Outlet and $15 \mathrm{~m} 1 \mathrm{les}$ upstream from lock 2 .

Records ava1lable.--U. S. Geolog1cal Survey: July 1938 to December 1953, da1ly gage he1ghts and discharges.

Gage.--Water-stage recorder. Datum of gage is at mean sea level, datum of 1929 (levels by Corps of Englneers). Prior to Jan. 17, 1952, at datum $1.44 \mathrm{ft}$ lower. Auxillary water-stage recorder a quarter of a mile upstream from Lake Hicpochee and 2.5 miles downstream from base gage. (All gage helghts tabulated below have been reduced to present datum, at mean sea level).

Stage-discharge relation.--Defined by current-meter measurements, affected by fall. D1scharge computed by using fall as determined by auxiliary water-stage recorder, as a factor.

Remarks.--Flow regulated by lock 1 at Lake Okeechobee. Since July 1951, records furn1shed by Corps of Engineers. 
Lake Okeechobee and the Everglades

(82) Caloosahatchee Canal at Moore Haven, Fla.--Continued

\begin{tabular}{|c|c|c|c|c|c|c|c|}
\hline $\begin{array}{l}\text { Calendar } \\
\text { year }\end{array}$ & Date & $\begin{array}{c}\text { Dally gage } \\
\text { helght } \\
\text { (feet) } \\
\text { (upper gage) }\end{array}$ & $\begin{array}{c}\text { Da1ly } \\
\text { d1scharge } \\
\text { (cfs) }\end{array}$ & $\begin{array}{c}\text { Calendar } \\
\text { year }\end{array}$ & Date & $\begin{array}{c}\text { Dally gage } \\
\text { helght } \\
\text { (feet) } \\
\text { (upper gage) }\end{array}$ & $\begin{array}{c}\text { Da1ly } \\
\text { discharge } \\
\text { (cfs) }\end{array}$ \\
\hline $\begin{array}{l}1939 \\
1940\end{array}$ & $\begin{array}{l}\text { Sept. } 9 \\
\text { Sept. } 16 \\
\text { Oct. } 11 \\
\text { Oct. } 13\end{array}$ & $\begin{array}{l}- \\
12.15 \\
13.22 \\
-\end{array}$ & $\begin{array}{c}3,310 \\
- \\
- \\
4,850\end{array}$ & $\begin{array}{l}1947 \\
1.948\end{array}$ & $\begin{array}{ll}\text { Sept. } 24 \\
\text { Dec. } 31 \\
\text { Sept. } 26 \\
\text { Oct. } 14\end{array}$ & $\begin{array}{l}14.87 \\
15.45\end{array}$ & $5, \overline{3} 20$ \\
\hline $\begin{array}{l}1941 \\
1942\end{array}$ & $\begin{array}{lr}\text { May } & 1 \\
\text { Oct.. } & 31 \\
\text { Mar. } & 14 \\
\text { July } & 8\end{array}$ & $\begin{array}{l}- \\
12.41 \\
12.62 \\
-\end{array}$ & $\begin{array}{c}4,890 \\
\overline{-} \\
5, \overline{3} 90\end{array}$ & $\begin{array}{l}1949 \\
1950\end{array}$ & $\begin{array}{ll}\text { Jan. } & 4 \\
\text { Jan. } & 1 \\
\text { Jan. } & 5\end{array}$ & $\begin{array}{c}13.11 \\
12.37 \\
-\end{array}$ & $\begin{array}{l}4,780 \\
4,410\end{array}$ \\
\hline 1943 & $\begin{array}{ll}\text { May } & 19 \\
\text { July } & 20\end{array}$ & 11.48 & 1,190 & 1951 & $\begin{array}{ll}\text { Oct. } & 20 \\
\text { Oct. } & 26\end{array}$ & 13.11 & $\begin{array}{c}4,500 \\
-\end{array}$ \\
\hline 1944 & $\begin{array}{ll}\text { Mar. } & 10 \\
\text { Sept. } & 17\end{array}$ & 21.39 & 1,140 & 1952 & $\begin{array}{lr}\text { Oct. } & 29 \\
\text { Nov. } & 6\end{array}$ & & $4, \overline{0} 60$ \\
\hline 1945 & $\begin{array}{l}\text { Sept. } \\
\text { Dec. } 8\end{array}$ & $\begin{array}{c}14.76 \\
-\end{array}$ & $5, \overline{660}$ & 1953 & $\begin{array}{ll}\text { Oct. } & 12 \\
\text { Dec. } & 22\end{array}$ & 14.20 & $4, \overline{770}$ \\
\hline 1946 & $\begin{array}{lr}\text { Mar. } & 19 \\
\text { Apr. } & 2\end{array}$ & 12.79 & $\begin{array}{c}4,150 \\
-\end{array}$ & & & & \\
\hline
\end{tabular}

(83) Orange Rlver near Fort Myers, Fla.

Location.--Lat $26^{\circ} 40^{\prime}$, Iong $81^{\circ} 43^{\prime}$, in sec. 9, T. 44 S., R. 26 E., $1 \frac{2}{2}$ miles southeast of Buckingham and 8 miles east of Fort Myers.

Dralnage area.- $-83.4 \mathrm{sq} \mathrm{m} 1$, approximately.

Records ava1lable.--U. S. Geologlcal Survey: November 1935 to October 1946, da1ly gage he1ghts and discharges. Prior to Oct. 1, 1941, published as "Twelvemile Creek near Fort Myers".

Gage.--Staff gage read twlce da1ly, and wooden control. Datum of gage is $1.71 \mathrm{ft}$ above mean sea Tevel, datum of 1929. Prior to Mar. 21, 1939, at site $50 \mathrm{ft}$ downstream at same datum and with no artificial controi.

Stage-discharge relation.--Defined by current-meter measurements below 2,800 cfs.

Annual peak stages and discharges

\begin{tabular}{|c|c|c|c|c|c|c|c|}
\hline $\begin{array}{l}\text { Calendar } \\
\text { year }\end{array}$ & Date & $\begin{array}{l}\text { Gage } \\
\text { height } \\
\text { (feet) }\end{array}$ & $\begin{array}{c}\text { Discharge } \\
\text { (cfs) }\end{array}$ & $\begin{array}{c}\text { Calendar } \\
\text { year }\end{array}$ & Date & $\begin{array}{l}\text { Gage } \\
\text { height } \\
\text { (feet) }\end{array}$ & $\begin{array}{c}\text { Discharge } \\
\text { (cfs) }\end{array}$ \\
\hline $\begin{array}{l}1936 \\
1937 \\
1938 \\
1939 \\
1940 \\
1941\end{array}$ & $\begin{array}{lr}\text { June } & 15 \\
\text { July } & 3 \\
\text { July } & 14 \\
\text { July } & 2 \\
\text { Sept. } & 12 \\
& \\
\text { July } & 17\end{array}$ & $\begin{array}{r}13.40 \\
7.70 \\
7.40 \\
9.00 \\
10.90 \\
8.38\end{array}$ & $\begin{array}{r}5,300 \\
850 \\
810 \\
733 \\
1,310 \\
842\end{array}$ & $\begin{array}{l}1942 \\
1943 \\
1944 \\
1945 \\
\\
1946\end{array}$ & $\begin{array}{ll}\text { Sept. } & 14 \\
\text { July } & 11 \\
\text { Aug. } & 15 \\
\text { July } & 23 \\
& \\
\text { Aug. } & 28\end{array}$ & $\begin{array}{l}6.40 \\
9.51 \\
6.62 \\
9.10 \\
7.58\end{array}$ & $\begin{array}{r}283 \\
1,440 \\
301 \\
1,200 \\
417\end{array}$ \\
\hline
\end{tabular}


Peace River Basin

(84) Drainage Canal west of Dundee, Fla.

Location.--Lat $28^{\circ} 01^{\prime}$, long $81^{\circ} 38^{\prime}$, in sec. 29 , T. 28 S., R. 27 E., on right bank at upstream slde of bridge on State H1ghway $542,1.2$ miles west of Dundee, and 1.4 miles downstream from Lake Ham1lton Outlet.

Dralnage area. $--50 \mathrm{sq} \mathrm{ml}$, approximately.

Records ava1lable.--U. S. Geological Survey: December 1946 to December 1953, da1ly gage heights and discharges.

Gage.--Water-stage recorder. Datum of gage is $114.08 \mathrm{ft}$ above mean sea level, datum of 1929. Jan. 25,1950 , to July 26,1951 , at site $150 \mathrm{ft}$ downstream at same datum.

Stage-discharge relation.--Defined by current-meter measurements. Considerable shifting at all stages.

Annual peak stages and discharges

\begin{tabular}{c|c|c|c||c|c|c|c}
\hline $\begin{array}{c}\text { Calendar } \\
\text { year }\end{array}$ & Date & $\begin{array}{c}\text { Gage } \\
\text { height } \\
\text { (feet) }\end{array}$ & $\begin{array}{c}\text { Discharge } \\
\text { (cfs) }\end{array}$ & $\begin{array}{c}\text { Calendar } \\
\text { year }\end{array}$ & Date & $\begin{array}{c}\text { Gage } \\
\text { height } \\
\text { (feet) }\end{array}$ & $\begin{array}{c}\text { Discharge } \\
\text { (cfs) }\end{array}$ \\
\hline 1947 & Sept. 18 & 6.86 & 188. & 1951 & Nov. 17 & 6.36 & 104 \\
1948 & Sept. 22 & 7.37 & 231 & 1952 & Oct. 20 & 6.99 & 104 \\
1949 & Aug. 27 oct. 4 & 6.77 & 203 & 1953 & Oct. & 6.88 & 222 \\
1950 & Oct. 18 or 19 & 6.41 & 116 & &
\end{tabular}

(85) Peace Creek Marsh outlet near Alturas, Fla.

Location.--Lat $27^{\circ} 55^{\prime}$, long $81^{\circ} 43^{\prime}$, in sec. 34, T. $29 \mathrm{~S} ., \mathrm{R} .26 \mathrm{E}$., near left bank at upstream slde of highway bridge half a mile north of State Highway $60,3.5$ miles north of Alturas, and $8 \frac{1}{4}$ miles east of Bartow.

Drainage area.--150 sq $\mathrm{ml}$, approximately.

Records avallable.--U. S. Geological Survey: January 1947 to December 1953, da1ly gage helghts and discharges.

Gage.--Water-stage recorder. Datum of gage is $97.67 \mathrm{ft}$ above mean sea level, datum of 1929 (State Road Department benchmark).

Stage-discharge relation.--Defined by current-meter measurements below 1,600 cfs.

H1storical data.--Maximum stage known, $13.3 \mathrm{ft}$ in 1928, from information by local resident.

Annual peak stages and discharges

\begin{tabular}{|c|c|c|c|c|c|c|c|}
\hline $\begin{array}{c}\text { Calendar } \\
\text { year }\end{array}$ & Date & $\begin{array}{c}\text { Gage } \\
\text { height } \\
\text { (feet) }\end{array}$ & $\begin{array}{c}\text { Discharge } \\
\text { (cfs) }\end{array}$ & $\begin{array}{c}\text { Calendar } \\
\text { year }\end{array}$ & Date & $\begin{array}{l}\text { Gage } \\
\text { height } \\
\text { (feet) }\end{array}$ & $\begin{array}{l}\text { Discharge } \\
\text { (cfs) }\end{array}$ \\
\hline 1928 & - & 13.3 & 2,540 & 1951 & July 30 , & 793 & 532 \\
\hline $\begin{array}{l}1947 \\
1948 \\
1949 \\
1950\end{array}$ & $\begin{array}{ll}\text { Sept. } & 19 \\
\text { Sept. } 23 \\
\text { Aug. } 28 \\
\text { Oct. } \quad 19\end{array}$ & $\begin{array}{r}10.88 \\
11.51 \\
11.67 \\
9.71\end{array}$ & $\begin{array}{r}1,360 \\
1,640 \\
1,740 \\
975\end{array}$ & $\begin{array}{l}1952 \\
1953\end{array}$ & $\begin{array}{ll}\text { Oct. } & 21 \\
\text { Oct. } & 10\end{array}$ & $\begin{array}{r}10.14 \\
9.90\end{array}$ & $\begin{array}{l}1,090 \\
1,020\end{array}$ \\
\hline
\end{tabular}




\section{Peace River Basin}

(86) Lake Howard at Winter Haven, Fla.

Location.--Lat $28^{\circ} \mathrm{O} \mathrm{I}^{\prime}$, Iong $81^{\circ} 44^{\prime}$, in NW $\frac{1}{4} \mathrm{sec}$. 29, T. $28 \mathrm{~S}$, R. 26 E., on c1ty dock and boat house near intersection of West Central Avenue and Lake Howard Drive in W1nter Haven.

Records ava1lable.--U. S. Geological Survey: June 1945 to February 1946, gage helghts read at perlodic intervals; February 1946 to December 1953, daily gage helghts.

Gage.-W-ter-stage recorder. Datum of gage is $120.00 \mathrm{ft}$ above mean sea level, datum of 1929. Prior to Feb. 13, 1946, staff gage at same site at datum $7.85 \mathrm{ft}$ higher.

Annual peak stages

\begin{tabular}{|c|c|c|c|c|c|}
\hline Calendar year & Date & $\begin{array}{l}\text { Gage helght } \\
\text { feet) }\end{array}$ & Calendar year & Date & $\begin{array}{l}\text { Gage height } \\
\text { feet) }\end{array}$ \\
\hline 1945 & July 15 & $a_{12.66}$ & 1950 & Oct. 18,21 & 11.95 \\
\hline $\begin{array}{l}1946 \\
1947 \\
1948 \\
1949\end{array}$ & $\begin{array}{l}\text { Aug. } \\
\text { Sept. } 23 \\
\text { Sept. } 28 \\
\text { Sept. } 29\end{array}$ & $\begin{array}{l}12.01 \\
12.46 \\
12.77 \\
12.57\end{array}$ & $\begin{array}{l}1951 \\
1952 \\
1953\end{array}$ & $\begin{array}{lr}\text { Oct. } & 2 \\
\text { Mar. } & 27 \\
\text { Oct. } & 9\end{array}$ & $\begin{array}{l}12.13 \\
12.24 \\
12.70\end{array}$ \\
\hline
\end{tabular}

aresent datum.

(87) Lulu Lake outlet at Elo1se, Fla.

Location.--Lat $27^{\circ} 59^{\prime}$, long $81^{\circ} 43^{\prime}$, in SE $\frac{1}{4}$ sec. 5, T. $29 \mathrm{~S}$. , R. 26 E., on left downstream abutment of culvert on State Highway 540-A at intersection with Rifle Range Road, 1 , 800 ft downstream from concrete control at outlet of Lulu Lake, and 0.8 mile southeast of Elo1se.

Drainage area.--26 sq $\mathrm{ml}$, approximately.

Records avallable.--U. S. Geological Survey: February 1946 to December 1953, da1ly gage helghts and discharges.

Gage.--Water-stage recorder. Datum of gage is $120.00 \mathrm{ft}$ above mean sea level, datum of 1929. Prior to Jan. 8, 1953, at site $1,500 \mathrm{ft}$ upstream at same datum.

Stage-discharge relation.--Poorly defined by current-meter measurements. Considerable shifting at all stages and backwater effect from hyacinths and aquatic vegetation.

Remarks.--Some regulation by Lulu Lake.

Annual peak stages and discharges

\begin{tabular}{|c|c|c|c|c|c|c|c|}
\hline $\begin{array}{c}\text { Calendar } \\
\text { year }\end{array}$ & Date & $\begin{array}{l}\text { Gage } \\
\text { height } \\
\text { (feet) }\end{array}$ & $\begin{array}{c}\text { Discharge } \\
\text { (cfs) }\end{array}$ & $\begin{array}{c}\text { Calendar } \\
\text { year }\end{array}$ & Date & $\begin{array}{l}\text { Gage } \\
\text { height } \\
\text { (feet) }\end{array}$ & $\begin{array}{c}\text { Discharge } \\
\text { (cfs) }\end{array}$ \\
\hline $\begin{array}{l}1946 \\
1947 \\
1948\end{array}$ & $\begin{array}{l}\text { Sept. } 26 \\
\text { Sept. } 19 \\
\text { Aug. } 25 \\
\text { Sept. } 24\end{array}$ & $\begin{array}{r}7.82 \\
9.56 \\
11.18 \\
-\end{array}$ & $\begin{array}{l}26 \\
52 \\
- \\
83\end{array}$ & $\begin{array}{l}1950 \\
1951 \\
1952 \\
\end{array}$ & $\begin{aligned} \text { oct. } & 18 \\
& 18 \\
\text { Sept. } 20, & 29,30 \\
\text { oct. } & 28\end{aligned}$ & $\begin{array}{r}8.93 \\
10.10 \\
9.40 \\
\end{array}$ & $\begin{array}{l}23 \\
35 \\
25 \\
\end{array}$ \\
\hline 1949 & $\begin{array}{l}\text { Aug. } 28 \text {, } \\
\text { Sept. } 29\end{array}$ & 10.53 & 72 & 1953 & oct. 10 & 10.33 & 86 \\
\hline
\end{tabular}




\section{Peace River Basin}

(88) Peace River at Bartow, Fla.

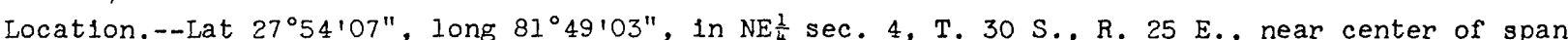
on downstream side of bridge on State H1ghway 60, $500 \mathrm{ft}$ downstream from McKinney Branch, and $0.6 \mathrm{mile}$ east of Bartow.

Drainage area. $--390 \mathrm{sq} \mathrm{ml}$, approximately.

Records available.--U. S. Geological Survey: November 1939 to December 1953, da1ly gage heights and discharges.

Gage.--Water-stage recorder. Datum of gage is $90.56 \mathrm{ft}$ above mean sea level, datum of 1929. Prior to July 12 , 1940, staff gage and July 12, 1940, to Nov. 5, 1948, water-stage recorder at site $200 \mathrm{ft}$ downstream at same datum.

Stage-discharge relation.--Defined by current-meter measurements below 2,900 cfs. Considerable shifting at all stages.

Annual peak stages and discharges

\begin{tabular}{|c|c|c|c|c|c|c|c|}
\hline $\begin{array}{c}\text { Calendar } \\
\text { year }\end{array}$ & Date & $\begin{array}{c}\text { Gage } \\
\text { height } \\
\text { (feet) }\end{array}$ & $\begin{array}{l}\text { Discharge } \\
\text { (cfs) }\end{array}$ & $\begin{array}{c}\text { Calendar } \\
\text { year }\end{array}$ & Date & $\begin{array}{c}\text { Gage } \\
\text { height } \\
\text { (feet) }\end{array}$ & $\begin{array}{c}\text { Discharge } \\
\text { (cfs) }\end{array}$ \\
\hline $\begin{array}{l}1940 \\
1941 \\
1942 \\
1943 \\
1944 \\
1945 \\
1946\end{array}$ & $\begin{array}{l}\text { Mar. } 23 \\
\text { Apr. } 6 \\
\text { July } 6 \\
\text { Aug. } 20,21 \\
\text { Oct. } 22 \\
\text { July } 26 \\
\text { Aug. } 3\end{array}$ & $\begin{array}{l}3.88 \\
4.73 \\
5.02 \\
5.07 \\
4.62 \\
5.80 \\
4.53\end{array}$ & $\begin{array}{r}638 \\
1,280 \\
1,300 \\
1,340 \\
1,010 \\
1,880 \\
1,060\end{array}$ & $\begin{array}{l}1947 \\
1948 \\
1949 \\
1950 \\
1951 \\
1952 \\
1953\end{array}$ & $\begin{array}{ll}\text { Sept. } & 24 \\
\text { Sept. } & 29 \\
\text { Aug. } & 30 \\
\text { Oct. } & 20 \\
& \\
\text { July } & 26 \\
\text { Oct. } 23, & 24 \\
\text { Oct. } & 11\end{array}$ & $\begin{array}{l}6.45 \\
6.13 \\
6.11 \\
5.40 \\
5.04 \\
5.73 \\
5.97\end{array}$ & $\begin{array}{l}4,140 \\
2,920 \\
2,850 \\
1,590 \\
1,210 \\
1,650 \\
2,030\end{array}$ \\
\hline
\end{tabular}

(89) Peace R1ver at Zolfo Springs, Fla.

Location.--Lat $27^{\circ} 30^{\prime}$, long $81^{\circ} 48^{\prime}$, in sec. 22, T. 34 S., R. 25 E., near right bank at downstream side of bridge on U. S. Highway $17,0.8$ mile north of Zolfo Springs.

Drainage area. $--840 \mathrm{sg} \mathrm{ml}$, approximately.

Records avallable.--U. S. Geologlcal Survey: September 1933 to December 1953, da1ly gage helghts and alscharges.

Gage.-Water-stage recorder. Datum of gage is $35.20 \mathrm{ft}$ above mean sea level, datum of 1929 .

Stage-discharge relation.--Defined by current-meter measurements below 22,500 cfs. Considerable shifting at medium and low water. 
Peace R1ver Basin

(89) Peace River at Zolfo Springs, Fla.--Continued

Annual peak stages and discharges

\begin{tabular}{|c|c|c|c|c|c|c|c|}
\hline $\begin{array}{c}\text { Calendar } \\
\text { year }\end{array}$ & Date & $\begin{array}{l}\text { Gage } \\
\text { height } \\
\text { (feet) }\end{array}$ & $\begin{array}{c}\text { Discharge } \\
\text { (cfs) }\end{array}$ & $\begin{array}{c}\text { Calendar } \\
\text { year }\end{array}$ & Date & $\begin{array}{c}\text { Gage } \\
\text { height } \\
\text { (feet) }\end{array}$ & $\begin{array}{c}\text { Discharge } \\
\text { (c/s) }\end{array}$ \\
\hline $\begin{array}{l}1933 \\
1934 \\
1935\end{array}$ & $\begin{array}{l}\text { Sept. } 6 \\
\text { June } 22 \\
\text { Sept. } 7\end{array}$ & $\begin{array}{l}20.05 \\
15.5 \\
13.20\end{array}$ & $\begin{array}{r}26,300 \\
9,850 \\
6,550\end{array}$ & $\begin{array}{l}1944 \\
1945\end{array}$ & $\begin{array}{l}\text { Aug. } \\
\text { June }\end{array}$ & $\begin{array}{r}8.48 \\
16.63\end{array}$ & $\begin{array}{r}2,600 \\
12,100\end{array}$ \\
\hline $\begin{array}{l}1936 \\
1937 \\
1938 \\
1939 \\
1940\end{array}$ & $\begin{array}{lr}\text { Feb. } & 18 \\
\text { July } & 3 \\
\text { Oct. } & 17 \\
\text { June } & 17 \\
\text { July } & 7\end{array}$ & $\begin{array}{r}11.82 \\
9.89 \\
15.15 \\
15.52 \\
9.17\end{array}$ & $\begin{array}{l}4,680 \\
3,440 \\
9,200 \\
9,800 \\
2,970\end{array}$ & $\begin{array}{l}1946 \\
1947 \\
1948 \\
1949 \\
1950\end{array}$ & $\begin{array}{l}\text { Sept. } 2 \\
\text { Sept. } 2 \\
\text { Sept. } 2 \\
\text { Aug. } \\
\text { Oct. } 2\end{array}$ & $\begin{array}{r}9.19 \\
19.15 \\
16.55 \\
18.11 \\
9.44\end{array}$ & $\begin{array}{r}3,030 \\
21,300 \\
11,600 \\
15,900 \\
3,370\end{array}$ \\
\hline $\begin{array}{l}1941 \\
1942 \\
1943\end{array}$ & $\begin{array}{lr} & \\
\text { July } & 17 \\
\text { June } & 13 \\
\text { Aug. } & 6 \\
\end{array}$ & $\begin{array}{l}10.98 \\
10.76 \\
10.63\end{array}$ & $\begin{array}{l}4,240 \\
4,180 \\
4,030\end{array}$ & $\begin{array}{l}1951 \\
1952 \\
1953\end{array}$ & $\begin{array}{ll}\text { Oct. } & \\
\text { Oct. } & 2 \\
\text { Aug. } & 3\end{array}$ & $\begin{array}{r}9.07 \\
14.70 \\
15.39\end{array}$ & $\begin{array}{l}3,150 \\
8,680 \\
9,300\end{array}$ \\
\hline
\end{tabular}

(90) Peace River at Arcad1a, Fla.

Location.--Lat $27^{\circ} 13^{\prime}$, long $81^{\circ} 52^{\prime}$, in sec. 26, T. 37 S., R. 24 E., on left bank 75 ft upstream from bridge on State H1ghway 70, half a m1le west of Arcadia, and about 5 m1les upstream from Joshua Creek.

Dra1nage area. $--1,370 \mathrm{sq} \mathrm{ml}$, approximately.

Records avallable.--U. S. Geolog1cal Survey: Apr11 1931 to December 1953, da1ly gage helghts and discharges.

Gage.--Woter-stage recorder. Datum of gage $1 \mathrm{~s} 8.25 \mathrm{ft}$ above mean sea level, datum of 1929. Prior to July 19, 1931, staff gage at same site and datum.

Stage-discharge relation.--Defined by current-meter measurements below $30,000 \mathrm{cfs}$.

Histor1cal data.--Maximum stage known, $18.3 \mathrm{ft}$ in 1912, from information by county eng1neer.

Annual peak stages and discharges

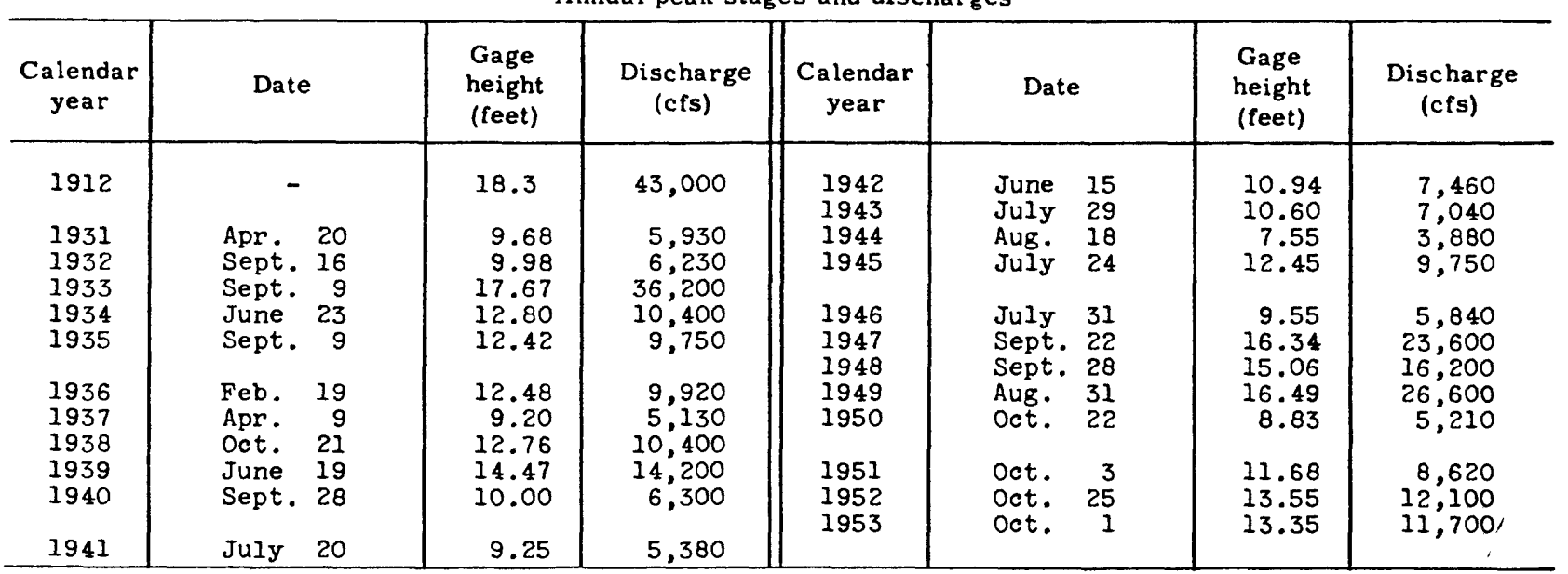


Miakka River Basin

(91) Miakka River near Sarasota, Fla.

Location.--La't $27^{\circ} 14^{\prime} 25^{\prime \prime}$, long $82^{\circ} 18^{\prime} 50^{\prime \prime}$, in sec. 21 , T. 37 S., R. 20 E., on right bank, half a mile upstream from bridge on State H1ghway 72,2 miles upstream from Lower Miakka Lake, and about 14 miles southeast of Sarasota.

Dra1nage area.--235 sq $\mathrm{ml}$, approximately.

Records ava1lable.--U. S. Geological Survey: August 1936 to December 1953, da1ly gage helghts and discharges.

Gage.--Staff gage read once da1ly. Datum of gage is $7.92 \mathrm{ft}$ above mean sea level, datum of 1929 . Prior to Apr. 10, 1941, at highway bridge at same datum.

Stage-discharge relation.--Defined by current-meter measurements below 6,600 cfs. Considerable shifting at all stages.

Annual peak stages and discharges

\begin{tabular}{|c|c|c|c|c|c|c|c|}
\hline $\begin{array}{c}\text { Calendar } \\
\text { year }\end{array}$ & Date & $\begin{array}{c}\text { Gage } \\
\text { height } \\
\text { (feet) }\end{array}$ & $\begin{array}{c}\text { Discharge } \\
\text { (cfs) }\end{array}$ & $\begin{array}{c}\text { Calendar } \\
\text { year }\end{array}$ & Date & $\begin{array}{c}\text { Gage } \\
\text { height } \\
\text { (feet) }\end{array}$ & $\begin{array}{c}\text { Discharge } \\
\text { (cfs) }\end{array}$ \\
\hline $\begin{array}{l}1937 \\
1938 \\
1939 \\
1940 \\
\end{array}$ & $\begin{array}{ll}\text { Apr. } & 9,10 \\
\text { Sept. } & 6 \\
\text { July } & 13 \\
\text { Oct. } 15,16 \\
\text { Aug. } 15 \\
\text { Sept. } 30 \\
\end{array}$ & $\begin{array}{c}- \\
7.65 \\
8.80 \\
- \\
8.52 \\
7.02\end{array}$ & $\begin{array}{c}1,340 \\
- \\
- \\
3,870 \\
4,040 \\
1,350 \\
\end{array}$ & \multirow{2}{*}{$\begin{array}{l}1945 \\
1946 \\
1947 \\
1948 \\
1949 \\
1950 \\
1951 \\
1952 \\
1953\end{array}$} & \multirow{2}{*}{$\begin{array}{lr}\text { Aug. } & 28 \\
& \\
\text { Aug. } & 5 \\
\text { Sept. } & 21 \\
\text { Sept. } & 30 \\
\text { Aug. } & 16 \\
\text { Sept: } \quad 9 \\
\\
\text { Oct. } \quad 4 \\
\text { Oct. } \quad 23,24 \\
\text { Oct. } \quad 12\end{array}$} & \multirow{2}{*}{$\begin{array}{r}8.68 \\
7.44 \\
10.78 \\
9.92 \\
8.54 \\
8.38 \\
9.24 \\
9.11 \\
8.67\end{array}$} & \multirow{2}{*}{$\begin{array}{l}3,360 \\
1,010 \\
6,620 \\
4,800 \\
3,160 \\
3,000 \\
3,440 \\
3,630 \\
2,520\end{array}$} \\
\hline $\begin{array}{l}1941 \\
1942 \\
1943 \\
1944\end{array}$ & $\begin{array}{ll}\text { July } & 20 \\
\text { Feb. } & 28 \\
\text { June } & 30, \\
\text { July } & 1,2 \\
\text { Aug. } & 8,9\end{array}$ & $\begin{array}{l}7.04 \\
7.12 \\
9.00 \\
6.16\end{array}$ & $\begin{array}{r}1,020 \\
1,190 \\
3,850 \\
268\end{array}$ & & & & \\
\hline
\end{tabular}

\section{Manatee R1ver Basin}

(92) Manatee River near Bradenton, Fla.

Location.--Lat $27^{\circ} 28^{\prime} 30^{\prime \prime}$, long $82^{\circ} 18^{\prime} 05^{\prime \prime}$, in sec. $34, \mathrm{~T}, 34 \mathrm{~S}, \mathrm{R}, 20 \mathrm{E}$, on left bank $20 \mathrm{ft}$ downstream from bridge on State Highway 675 , $800 \mathrm{ft}$ upstream from Craig Branch, $6 \frac{1}{4} \mathrm{~m} 11 \mathrm{es}$ northwest of Verna, and 17 miles east of Bradenton.

Drainage area. $--90 \mathrm{sq} \mathrm{ml}$, approximately.

Records ava1lable.--U. S. Geological Survey: Apr1l 1939 to December 1953, da1ly gage helghts and discharges.

Gage.--Water-stage recorder. Datum of gage is $11.72 \mathrm{ft}$ above mean sea level, datum of 1929.

Stage-discharge relation.--Defined by current-meter measurements below 3,400 cfs. Cons1derable shifting at medium and low water. 
Manatee River Basin

(92) Manatee River near Bradenton, Fla.--Cont1nued

A nnual peak stages and discharges



Little Manatee R1ver Basin

(93) Little Manatee River near Wimauma, Fla.

Iocation.--Lat $27^{\circ} 40^{\prime} 15^{\prime \prime}$, long $82^{\circ} 21 \cdot 10^{\prime \prime}$, In sec. 25, T. 32 S., R. I9 E., on left bank $25 \mathrm{ft}$

downstream from bridge on U. S. Highway 301 , li m1les upstream from Cypress Creek, and $4 \mathrm{mlles}$ southwest of Wimauma.

Dralnage area.--145 sq $\mathrm{ml}$, approximately.

Fecords avallable.--U. S. Geological Survey: March 1939 to December 1953, da1ly gage he1ghts and discharges.

Gage.-Water-stage recorder. Datum of gage $1 \mathrm{~s} 2.17 \mathrm{ft}$ above mean sea level, datum of 1929. Stage-d1scharge relation.--Defined by current-meter measurements below 7,200 cfs.

Annual peak stages and discharges

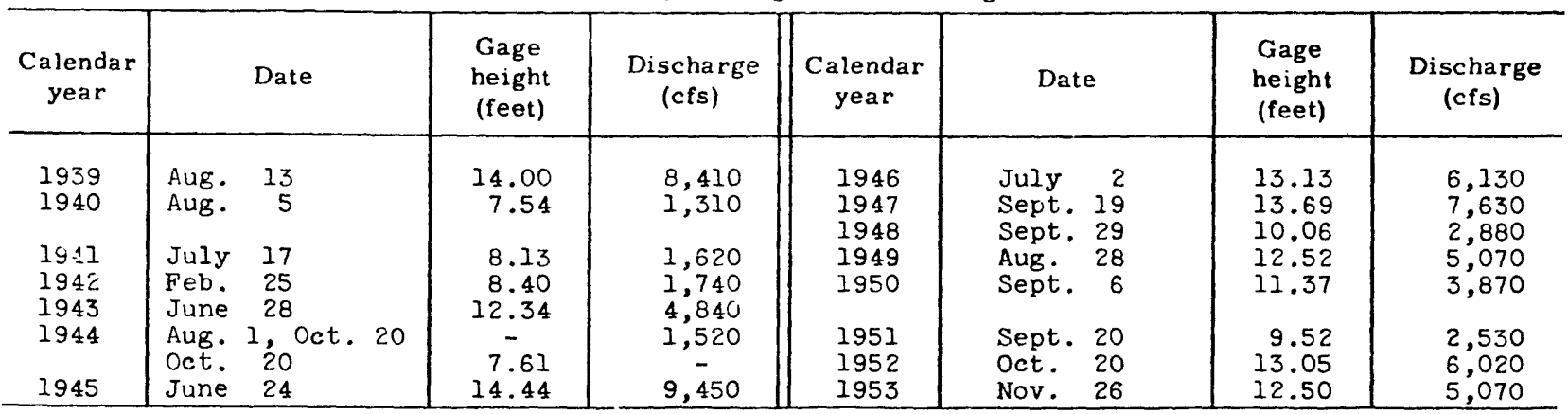




\section{Alafia River Basin}

(94) Alafla River at Lithia, Fla.

Location.--Lat $27^{\circ} 52^{\prime}$, long $82^{\circ} 12^{\prime}$, in sec. 16, T. $30 \mathrm{~S}$., R. $21 \mathrm{E}$., on left bank $11 \mathrm{ft}$ downstream from Marvinia Bridge on highway, I mile northwest of Lithia, and $1 \frac{1}{4} \mathrm{miles}$ downstream from Little Alafia River.

Drainage area.--335 sq $\mathrm{ml}$, approximately.

Records avallable.--U. S. Geologlcal Survey: January 1933 to December 1953, da1ly gage he1ghts and discharges.

Gage.--Water-stage recorder. Datum of gage is $9.86 \mathrm{ft}$ above mean sea level, datum of 1929. Prior to Aug. 8, 1939 , staff gage at site $250 \mathrm{ft}$ upstream at same datum.

Stage-discharge relation.--Defined by current-meter measurements below 10,000 cfs. Considerable shifting in lower limits of rating curve.

Annual peak stages and discharges

\begin{tabular}{|c|c|c|c|c|c|c|c|}
\hline $\begin{array}{c}\text { Calendar } \\
\text { year }\end{array}$ & Date & $\begin{array}{l}\text { Gage } \\
\text { height } \\
\text { (feet) }\end{array}$ & $\begin{array}{l}\text { Discharge } \\
\text { (cfs) }\end{array}$ & $\begin{array}{l}\text { Calendar } \\
\text { year }\end{array}$ & Date & $\begin{array}{l}\text { Gage } \\
\text { height } \\
\text { (feet) }\end{array}$ & $\begin{array}{l}\text { Discharge } \\
\text { (cfs) }\end{array}$ \\
\hline $\begin{array}{l}1933 \\
1934 \\
1935\end{array}$ & $\begin{array}{lr}\text { Sept. } & 7 \\
\text { June } & 16 \\
\text { Sept. } & 6\end{array}$ & $\begin{array}{l}25.6 \\
16.40 \\
18.08\end{array}$ & $\begin{array}{r}19,300 \\
6,410 \\
8,300\end{array}$ & $\begin{array}{l}1944 \\
1945\end{array}$ & $\begin{array}{ll}\text { Oct. } & 20 \\
\text { July } & 26\end{array}$ & 11.45 & $\begin{array}{l}2,540 \\
6,850\end{array}$ \\
\hline $\begin{array}{l}1936 \\
1937 \\
1938 \\
1939 \\
1940\end{array}$ & $\begin{array}{lr}\text { Feb. } & 11 \\
\text { Apr. } & 7 \\
\text { Oct. } & 17 \\
\text { June } & 17 \\
\text { Feb. } & 19\end{array}$ & $\begin{array}{r}11.80 \\
9.60 \\
16.85 \\
13.90 \\
6.84\end{array}$ & $\begin{array}{l}2,420 \\
1,710 \\
6,870 \\
3,720 \\
1,140\end{array}$ & $\begin{array}{l}1946 \\
1947 \\
1948 \\
1949 \\
1950\end{array}$ & $\begin{array}{lr}\text { July } & 31 \\
\text { Sept. } & 20 \\
\text { Sept. } & 30 \\
\text { Aug. } & 29 \\
\text { Sept. } & 7\end{array}$ & $\begin{array}{l}10.60 \\
20.38 \\
15.59 \\
19.28 \\
15.04\end{array}$ & $\begin{array}{r}2,220 \\
11,100 \\
5,590 \\
9,740 \\
5,030\end{array}$ \\
\hline $\begin{array}{l}1941 \\
1942 \\
1943\end{array}$ & $\begin{array}{ll}\text { July } & 16 \\
\text { Mar. } & 20 \\
\text { June } & 28\end{array}$ & $\begin{array}{r}13.66 \\
9.23 \\
16.14\end{array}$ & $\begin{array}{l}4,050 \\
1,760 \\
6,390\end{array}$ & $\begin{array}{l}1951 \\
1952 \\
1953\end{array}$ & $\begin{array}{ll}\text { Oct. } & 2 \\
\text { Oct. } 21 & \\
\text { Nov. } & 25 \text { or } 26\end{array}$ & $\begin{array}{r}8.41 \\
16.27 \\
14.38\end{array}$ & $\begin{array}{l}1,550 \\
6,280 \\
4,420\end{array}$ \\
\hline
\end{tabular}

Hillsborough River Basin

(95) H1llsborough River near Zephyrhilis, Fla.

Location.--Lat $28^{\circ} \mathrm{Og} \mathrm{\prime}$, long $82^{\circ} 14^{\prime}$, in sec. 8 , T. $27 \mathrm{~S}$. , R. $21 \mathrm{E}$, on left bank $10 \mathrm{ft}$ downstream from footbridge in Hillsborough River State Park, 2 miles downstream from Blackwater Creek, and 7 miles southwest of Zephyrhilis.

Drainage area.--220 sq $\mathrm{ml}$, approximately.

Records ava1lable.--U. S. Geological Survey: November 1939 to December 1953, da1ly gage helghts and discharges.

Gage.--Staff gage read once daily. Datum of gage is $33.28 \mathrm{ft}$ above mean sea level (Corps of Engineers benchmark).

Stage-discharge relation.--Defined by current-meter measurements. 
H11lsborough R1ver Basin

(95) H1llsborough R1ver near Zephyrh111s, Fla.--Cont1nued

Annual peak stages and discharges

\begin{tabular}{|c|c|c|c|c|c|c|c|}
\hline $\begin{array}{c}\text { Calendar } \\
\text { year }\end{array}$ & Date & $\begin{array}{c}\text { Gage } \\
\text { height } \\
\text { (feet) }\end{array}$ & $\begin{array}{c}\text { Discharge } \\
\text { (cfs) }\end{array}$ & $\begin{array}{c}\text { Calendar } \\
\text { year }\end{array}$ & Date & $\begin{array}{l}\text { Gage } \\
\text { height } \\
\text { (feet) }\end{array}$ & $\begin{array}{c}\text { Dischargo } \\
\text { (cfa) }\end{array}$ \\
\hline $\begin{array}{l}1940 \\
1941 \\
1942 \\
1943 \\
1944 \\
1945 \\
1946\end{array}$ & $\begin{array}{l}\text { Feb. } 18 \\
\text { Apr. } 4 \\
\text { Mar. } 3 \\
\text { Aug. } 31 \\
\text { Oct. } 20 \\
\text { July } 26 \\
\text { Aug. } 2\end{array}$ & $\begin{array}{r}4.84 \\
12.65 \\
8.72 \\
10.23 \\
8.20 \\
13.30 \\
9.40\end{array}$ & $\begin{array}{r}724 \\
4,230 \\
1,760 \\
2,350 \\
1,590 \\
5,330 \\
2,010 \\
\end{array}$ & $\begin{array}{l}1947 \\
1948 \\
1949 \\
1950 \\
1951 \\
1952 \\
1953\end{array}$ & $\begin{array}{lr}\text { Sept. } & 19 \\
\text { Jan. } & 25 \\
\text { Aug. } & 28 \\
\text { Sept. } & 7 \\
\text { Sept. } & 19 \\
\text { Mar. } & 27 \\
\text { Sept. } & 28\end{array}$ & $\begin{array}{r}13.71 \\
12.02 \\
12.90 \\
13.80 \\
6.18 \\
7.60 \\
12.66\end{array}$ & $\begin{array}{l}5,920 \\
3,600 \\
4,620 \\
5,890 \\
1,060 \\
1,420 \\
4,310\end{array}$ \\
\hline
\end{tabular}

(96) H1llsborough River near Tampa, Fla.

Location.--Lat $28^{\circ} 01^{\prime} 25^{\prime \prime}$, long $82^{\circ} 25^{\prime} 40^{\prime \prime}$, In sec. 29, T. $28 \mathrm{~S} ., \mathrm{R}, 19 \mathrm{E}$. , on left bank just upstream from splilway of Tampa reservolr dam, at Thirtieth street, $5 \frac{1}{2}$ miles northeast or Tampa.

Drainage area.--650 8q $\mathrm{ml}$, approximately.

Records avallable.--U. S. Qeolog10al Survey: Ootober 1933 to December 1939, da1ly gage he1ghts and discharges at former station upstream published as "H1llsborough RIver near Harney". October 1938 to Deoember 1953, da1ly gage he1ghts and discharges.

Gage.--Water-stage reoorder. Datum of gage 1s at mean sea level, datum of 1929 (01ty or Tampa benchmark). Dot. 1, 1938, to Sept. 30, 1945, at Fortleth Street Bridge 1.4 miles upetream at datum 0.66 st higher.

oot. 17 1933, to Deo. 31, 1939, starf gage at Fowler Street Bridge, 2t miles north or Harney, and about 81 m1les upstream from present site. Datum of th16 gage was 19.14 ft above mean sea level (levels by HIl sborough County Engineering Department).

Stage-d1soharge relat1on.--At s1tos prior to 1945 when the reservolr dam was oomploted, stagedisoharge relation were der Ined by ourrent-meter measurements below 16,400 of. Since 1045 , hoad-disoharge relation and rating of taintor gates and flashboards on orest of dam have been defined by ourrent-meter measurements.

H1storioal data.--Maximum stage known, about $15.5 \mathrm{ft}$ sept, 9,1933 , at ite and datum or Harney

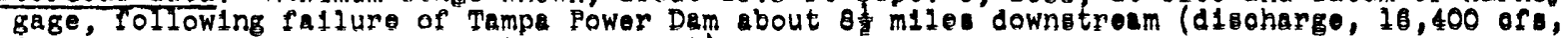
from current-meter melsurement near orest).

Remarks.--Flow regulated by Tampa reservolr dam inae oot. $1,1945$.

Annual peak atageg and discharges

\begin{tabular}{|c|c|c|c|c|c|c|c|}
\hline $\begin{array}{c}\text { Calondar } \\
\text { year }\end{array}$ & Date & $\begin{array}{c}\text { Gage } \\
\text { height } \\
\text { (faet) }\end{array}$ & $\begin{array}{l}\text { Digoharge } \\
\text { (ofe) }\end{array}$ & $\begin{array}{c}\text { Calendar } \\
\text { year }\end{array}$ & Dato & $\begin{array}{l}\text { Gage } \\
\text { height } \\
\text { (fe日t) }\end{array}$ & $\begin{array}{l}\text { Diseharge } \\
\text { (eff) }\end{array}$ \\
\hline $\begin{array}{l}1933 \\
1934 \\
1935\end{array}$ & $\begin{array}{l}\text { Sept. } 8 \\
\text { June } 20 \\
\text { Sept. } 8\end{array}$ & $\begin{array}{l}15.5 \\
13.42 \\
10.49\end{array}$ & $\begin{array}{r}16,400 \\
11,700 \\
3,550\end{array}$ & $\begin{array}{r}1844 \\
1845 \\
\end{array}$ & $\begin{array}{ll}\text { Oet, } & 20 \\
\text { JuIy } & 28,20 \\
\end{array}$ & $\begin{array}{r}1.83 \\
20.85 \\
\end{array}$ & $\begin{array}{r}1,780 \\
9,890 \\
\end{array}$ \\
\hline $\begin{array}{l}1936 \\
1937 \\
1938 \\
1939 \\
1940\end{array}$ & $\begin{array}{l}\text { Fob, } 19 \\
\text { Sept. } 3,4 \\
\text { oot. } 19 \\
\text { JuIy } \frac{12}{\text { Fob. } 22}\end{array}$ & $\begin{array}{r}7.32 \\
3.12 \\
6.82 \\
71.74 \\
0.46\end{array}$ & $\begin{array}{r}3,530 \\
1,960 \\
2,740 \\
2,900 \\
1,300\end{array}$ & $\begin{array}{l}1946 \\
1947 \\
1948 \\
7949 \\
1950\end{array}$ & 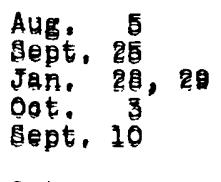 & $=$ & 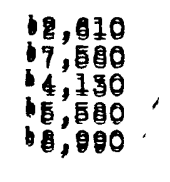 \\
\hline $\begin{array}{l}1941 \\
1942 \\
\end{array}$ & $\begin{array}{ll}\text { Apr. } & \\
\text { Mar. } & 8-10 \\
\end{array}$ & $\begin{array}{r}12.81 \\
9.08 \\
\end{array}$ & $\begin{array}{r}4,500 \\
2,230 \\
\end{array}$ & $\begin{array}{l}1951 \\
1962 \\
1853 \\
\end{array}$ & $\begin{array}{l}\text { Oet, } \quad 4 \\
\text { Apr: } \\
\text { 3ept. } 30 \\
\end{array}$ & $=$ & $\begin{array}{r}1,550 \\
1,000 \\
18,030 \\
\end{array}$ \\
\hline
\end{tabular}

- Following rallure of power dem on sept. 7 .

- Maximum dafiy disoharga (reguiated). 


\section{Lake Tarpon Bas1n}

(97) Brooker Creek near Odessa, Fla.

Location.--Lat $28^{\circ} 08^{\prime} 05^{\prime \prime}$, long $82^{\circ} 35^{\prime} 40^{\prime \prime}$, in sec. 10, T. $27 \mathrm{~S} ., \mathrm{R} .17 \mathrm{E}$, on left bank $20 \mathrm{ft}$ upstream from bridge on State H1ghway $232,30 \mathrm{ft}$ downstream from outlet of Keystone Lake, and 3.2 miles south of Odessa.

Drainage area.--10 sq $\mathrm{ml}$, approximately.

Records available.--U. S. Geological Survey: Apr1l 1946 to December 1953, da1ly gage helghts and discharges.

Gage.--Water-stage recorder. Datum of gage $1 \mathrm{~s} 30.00 \mathrm{ft}$ above mean sea level, datum of 1929. Stage-discharge relation.--Poorly defined by current-meter measurements below 124 cfs. Considerable shlfting at all stages.

Annual peak stages and discharges

\begin{tabular}{|c|c|c|c|c|c|c|c|}
\hline $\begin{array}{c}\text { Calendar } \\
\text { year }\end{array}$ & Date & $\begin{array}{l}\text { Gage } \\
\text { height } \\
\text { (feet) }\end{array}$ & $\begin{array}{c}\text { Discharge } \\
\text { (cfs) }\end{array}$ & $\begin{array}{c}\text { Calendar } \\
\text { year }\end{array}$ & Date & $\begin{array}{l}\text { Gage } \\
\text { height } \\
\text { (feet) }\end{array}$ & $\begin{array}{l}\text { Discharge } \\
\text { (cfs) }\end{array}$ \\
\hline $\begin{array}{l}1946 \\
1947 \\
1948 \\
1949 \\
1950\end{array}$ & $\begin{array}{ll}\text { Oct. } & 11,12 \\
\text { Aug. } & 21 \\
\text { Feb. } & 1 \\
\text { Aug. } & 23 \\
\text { Sept. } 8 & \end{array}$ & $\begin{array}{l}11.88 \\
12.62 \\
11.61 \\
13.20 \\
12.87\end{array}$ & $\begin{array}{r}33 \\
65 \\
23 \\
180 \\
130 \\
\end{array}$ & $\begin{array}{l}1951 \\
1952 \\
1953\end{array}$ & $\begin{array}{l}\text { Oct. } 2,3 \\
\text { Apr. } 1 \\
\text { Oct. } 26 \\
\text { Oct. } 1\end{array}$ & $\begin{array}{c}10.73 \\
- \\
11.44 \\
12.60\end{array}$ & $\begin{array}{l}5.8 \\
23 \\
73\end{array}$ \\
\hline
\end{tabular}

\section{Anclote River Basin}

(98) Anclote Rlver near Elfers, Fla.

Location.--Lat $28^{\circ} 12^{\prime} 50^{\prime \prime}$, long $82^{\circ} 40^{\prime} 00^{\prime \prime}$, in sec. 23 T. 26 S., R. 16 E., on left bank $40 \mathrm{ft}$ downstream from bridge on State Highway 54 , and $3 \frac{1}{2}$ miles southeast of Elfers.

Dra1nage area. $--67 \mathrm{sq} \mathrm{m} 1$, approximately.

Records avallable.--U. S. Geological Survey: May 1946 to December 1953, da1ly gage heights and discharges.

Gage.--Water-stage recorder. Datum of gage is at mean sea level, datum of 1929.

Stage-discharge relation.--Defined by current-meter measurements below 3,200 cfs. Slight shift in stage-discharge relation following channel changes and construction of new bridge in 1951.

Mistorlcal data.-Flood of Aug. 8 or 9 , 1945, reached a stage of 27.7 ft, from information by local residents and high-water marks (discharge, 5,000 cfs, from rating curve extended above $3,200 \mathrm{cfs})$.

\begin{tabular}{|c|c|c|c|c|c|c|c|}
\hline $\begin{array}{c}\text { Calendar } \\
\text { year }\end{array}$ & Date & $\begin{array}{l}\text { Gage } \\
\text { height } \\
\text { (feet) }\end{array}$ & $\begin{array}{c}\text { Discharge } \\
\text { (cfs) }\end{array}$ & $\begin{array}{c}\text { Calendar } \\
\text { year }\end{array}$ & Date & $\begin{array}{c}\text { Gage } \\
\text { height } \\
\text { (feet) }\end{array}$ & $\begin{array}{l}\text { Discharge } \\
\text { (cfs) }\end{array}$ \\
\hline 1945 & Aug. 8 or 9 & 27.7 & 5,000 & 1950 & Sept. 6 & 26.02 & 3,500 \\
\hline $\begin{array}{r}1946 \\
1947 \\
1948 \\
1949 \\
\end{array}$ & $\begin{array}{ll}\text { Oct. } & 10 \\
\text { Sept. } & 20 \\
\text { Jan. } & 26 \\
\text { Aug. } & 22\end{array}$ & $\begin{array}{l}19.55 \\
21.76 \\
20.54 \\
23.02\end{array}$ & $\begin{array}{r}866 \\
1,160 \\
974 \\
1,550\end{array}$ & $\begin{array}{l}1951 \\
1952 \\
1953\end{array}$ & $\begin{array}{ll}\text { July } & 30 \\
\text { Mar. } & 28 \\
\text { Apr. } & 14\end{array}$ & $\begin{array}{l}14.81 \\
19.36 \\
24.37\end{array}$ & $\begin{array}{r}457 \\
881 \\
2,340\end{array}$ \\
\hline
\end{tabular}




\section{W1thlacoochee R1ver Basin}

(99) Withlacoochee River at Trilby, Fla.

Location. - Lat $28^{\circ} 29^{\prime}$, long $82^{\circ} 11^{\prime}$, on line between secs. 14 and 23, T. 23 S., R. 21 E.,

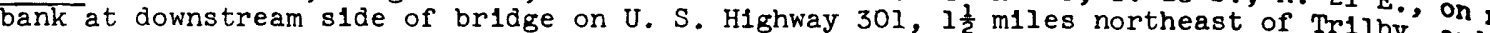
10 miles upstream from Little Withlacoochee R1ver.

Drainage area.--650 sq $\mathrm{m} 1$, approximately. Records avallable.--U. S. Geologlcal Survey: August 1928 to February 1929, February 1930 to
December 1953, da1iy gage helghts and discharges.

Gage.--Water-stage recorder. Datum of gage is $49.27 \mathrm{ft}$ above mean sea level (Corps of Englneer benchmark). Prior to 0ct. 1, 1938, staff gage at site $1 \frac{1}{2}$ miles downstream at datum 0.12 ft lower.

Stage-discharge relation.--Defined by current-meter measurements .

H1storical data.--See information in regard to 1933 and 1934 floods for station at Croom.

Annual peak stages and discharges

\begin{tabular}{|c|c|c|c|c|c|c|c|}
\hline $\begin{array}{c}\text { Calendar } \\
\text { year }\end{array}$ & Date & $\begin{array}{l}\text { Gage } \\
\text { height } \\
\text { (feet) }\end{array}$ & $\begin{array}{l}\text { Discharge } \\
\text { (cfs) }\end{array}$ & $\begin{array}{c}\text { Calendar } \\
\text { year }\end{array}$ & Date & $\begin{array}{l}\text { Gage } \\
\text { height } \\
\text { (feet) }\end{array}$ & $\begin{array}{l}\text { Discharge } \\
\text { (cfs) }\end{array}$ \\
\hline 1928 & Oct. 3,4 & 15.35 & 3,340 & 1941 & July 26,27 & 11.93 & 1,790 \\
\hline 1930 & Apr. 10 & 12.22 & 2,070 & $\begin{array}{l}1942 \\
1943 \\
1944\end{array}$ & $\begin{array}{ll}\text { Mar. } & 12 \\
\text { Aug. } & 23,24 \\
\text { Oct. } & 29\end{array}$ & $\begin{array}{r}10.04 \\
9.93 \\
11.10\end{array}$ & $\begin{array}{l}1,190 \\
1,070 \\
1,510\end{array}$ \\
\hline $\begin{array}{l}1931 \\
1932\end{array}$ & $\begin{array}{l}\text { Apr. } 19 \\
\text { Sept. } 17\end{array}$ & $\begin{array}{r}10.75 \\
4.04\end{array}$ & $\begin{array}{r}1,590 \\
354\end{array}$ & 1945 & July 31 & 14.20 & 2,830 \\
\hline $\begin{array}{l}1933 \\
1934\end{array}$ & & $\begin{array}{l}20.18 \\
20.5\end{array}$ & $\begin{array}{l}8,300 \\
8,840\end{array}$ & $\begin{array}{l}1946 \\
1947\end{array}$ & $\begin{array}{l}\text { Aug. } 6 \\
\text { Sept. } 30\end{array}$ & $\begin{array}{r}8.93 \\
12.86\end{array}$ & $\begin{array}{r}853 \\
2.050\end{array}$ \\
\hline 1935 & Sept. 16 & 12.58 & 2,270 & 1948 & Aug. 23 & 13.38 & 2,250 \\
\hline $\begin{array}{l}1936 \\
1937\end{array}$ & $\begin{array}{l}\text { Feb. } 29 \\
\text { Sept. } \quad 3\end{array}$ & $\begin{array}{l}10.30 \\
11.55\end{array}$ & $\begin{array}{l}1,360 \\
1,760\end{array}$ & 1950 & Sept. I1 & 17.93 & 4,790 \\
\hline $\begin{array}{l}1938 \\
1939 \\
1940\end{array}$ & $\begin{array}{lr}\text { Oct. } & 27 \\
\text { Sept. } & 6 \\
\text { July } & 7\end{array}$ & $\begin{array}{r}8.67 \\
12.59 \\
9.28\end{array}$ & $\begin{array}{r}764 \\
1,970 \\
902\end{array}$ & $\begin{array}{l}1951 \\
1952 \\
1953\end{array}$ & $\begin{array}{ll}\text { Oct. } & 9,10 \\
\text { Apr. } & 7 \\
\text { Sept. } & 19\end{array}$ & $\begin{array}{r}10.88 \\
9.15 \\
13.79\end{array}$ & $\begin{array}{r}1,400 \\
900 \\
2.420\end{array}$ \\
\hline
\end{tabular}

(100) W1thlacoochee River at Croom, Fla.

Location.--Lat $28^{\circ} 36^{\prime}$, long $82^{\circ} 13^{\prime}$, in sec. 8, T. 22 S., R. 21 E., on left bank at upstream side of old county highway bridge at'Croom, 2 miles downstream from Little Withlacoochee River.

Drainage area.--900 sq $\mathrm{ml}$, approximately.

Records avallable.--U. S. Geologlcal Survey: November 1939 to December 1953, da1ly gage he1ghts and discharges.

Gage.-Water-stage recorder. Datum of gage 1s $38.94 \mathrm{ft}$ above mean sea level (Corps of Eng1neers benchmark). Prior to Feb. 2, 1940, staff gage at ra1lroad bridge $500 \mathrm{ft}$ upstream at same datum.

Stage-discharge relation.--Defined by current-meter measurements.

Historical data.--A former observer at this station obtalned the information from long-time local residents that the flood of september 1933 reached the bridge floor and the flood of June 1934 was it had been at least 75 years since a stage even approaching the 1933 and 1934 stages had occurred. 
Withlacoochee River Basin

(100) Withlacoochee River at Croom, Fla.--Continued

Annual peak stages and discharges

\begin{tabular}{c|c|c|c|c|c|c|c}
\hline $\begin{array}{c}\text { Calendar } \\
\text { year }\end{array}$ & Date & $\begin{array}{c}\text { Gage } \\
\text { height } \\
\text { (feet) }\end{array}$ & $\begin{array}{c}\text { Discharge } \\
\text { (cfs) }\end{array}$ & $\begin{array}{c}\text { Calendar } \\
\text { year }\end{array}$ & Date & $\begin{array}{c}\text { Gage } \\
\text { height } \\
\text { (feet) }\end{array}$ & $\begin{array}{c}\text { Discharge } \\
\text { (cfs) }\end{array}$ \\
\hline 1940 & July 12 & 7.45 & 1,080 & 1947 & Sept. 28, 29 & 9.67 & 3,160 \\
1941 & Apr. 12 & 8.62 & 2,060 & 1948 & Aug. 25 & 9.62 & 3,100 \\
1942 & Mar. 18 & 7.92 & 1,400 & 1949 & Sept. 3 & 9.97 & 3,330 \\
1943 & Aug. 25 & 7.90 & 1,400 & 1,700 & 12.71 & 8,450 \\
1944 & Oct. 29,30 & 8.56 & 1,800 & 1951 & Oct. 11 & 8.00 & 1,390 \\
1945 & July 29 & 10.44 & 4,290 & 1952 & Apr. 7 & 7.39 & 1,070 \\
1946 & Aug. 6 & 7.58 & 1,180 & 1953 & Sept. 22, 23 & 10.12 & 3,560 \\
\hline
\end{tabular}

(101) Withlacoochee River near Holder, Fla.

Location.--Lat $28^{\circ} 59^{\prime} 15^{\prime \prime}$, long $82^{\circ} 20^{\prime} 50^{\prime \prime}$, in sec. 19, T. $17 \mathrm{~S} ., \mathrm{R}, 20 \mathrm{E}$, on right bank, $100 \mathrm{ft}$ downstream from bridge on State Highway $200,4 \frac{1}{2}$ miles northeast of Holder.

Drainage area. $--1,710 \mathrm{sq} \mathrm{mi}$, approximațely.

Records ava1lable.--U. S. Geological Survey: August 1928 to February 1929, August 1931 to December 1953, da1ly gage helghts and discharges.

Gage.--Water-stage recorder. Datum of gage $1 \mathrm{~s} 27.52 \mathrm{ft}$ above mean sea level (levels by Corps of Englneers). Aug. 14, 1928, to Feb. 15, 1929, staff gage at bridge $100 \mathrm{ft}$ upstream at datum $2.00 \mathrm{ft}$ higher.

Stage-discharge relation.--Defined by current-meter measurements.

Annual peak stages and discharges

\begin{tabular}{|c|c|c|c|c|c|c|c|}
\hline $\begin{array}{c}\text { Calendar } \\
\text { year }\end{array}$ & Date & $\begin{array}{c}\text { Gage } \\
\text { height } \\
\text { (feet) }\end{array}$ & $\begin{array}{l}\text { Discharge } \\
\quad \text { (cfs) }\end{array}$ & $\begin{array}{c}\text { Calendar } \\
\text { year }\end{array}$ & Date & $\begin{array}{l}\text { Gage } \\
\text { height } \\
\text { (feet) }\end{array}$ & $\begin{array}{l}\text { Discharge } \\
\quad \text { (cfs) }\end{array}$ \\
\hline $\begin{array}{l}1928 \\
1931 \\
1932 \\
1933 \\
1934 \\
1935 \\
1936 \\
1937 \\
1938 \\
1939 \\
1940 \\
1941 \\
\end{array}$ & $\begin{array}{l}\text { Oct. } 16 \\
\text { Sept. } 19 \\
\text { Sept. } 9 \\
\text { Sept. } 26 \\
\text { July } 9,10 \\
\text { Sept. } 30 \\
\text { Mar. } 17 \\
\text { Aug. } 31 \\
\text { Nov. } 6 \\
\text { Sept. } 25 \\
\text { Aug. } 8,9 \\
\text { Apr. } 22\end{array}$ & $\begin{array}{r}a 11.26 \\
4.36 \\
- \\
11.17 \\
11.63 \\
8.86 \\
7.66 \\
8.46 \\
5.48 \\
7.94 \\
4.76 \\
5.90\end{array}$ & $\begin{array}{l}5,830 \\
1,210 \\
555 \\
5,860 \\
6,740 \\
3,290 \\
2,800 \\
3,090 \\
1,560 \\
2,720 \\
1,400 \\
1,890\end{array}$ & $\begin{array}{l}1942 \\
1943 \\
1944 \\
1945 \\
1946 \\
1947 \\
1948 \\
1949 \\
1950 \\
1951 \\
1952 \\
1953\end{array}$ & $\begin{array}{l}\text { Mar. } 28 \\
\text { Sept. } 2 \\
\text { Nov. } 10 \\
\text { Aug. } 20 \\
\text { Oct. } 12 \\
\text { Oct. } 16,17 \\
\text { Sept. } 2 \\
\text { Oct. } 2 \\
\text { Sept. } 28 \\
\text { Oct. } 23 \\
\text { Mar. } 30,31 \\
\text { Oct. } 9\end{array}$ & $\begin{array}{r}6.13 \\
7.86 \\
6.36 \\
10.54 \\
7.55 \\
8.73 \\
9.49 \\
10.36 \\
10.40 \\
4.10 \\
5.25 \\
10.46\end{array}$ & $\begin{array}{l}1,900 \\
2,780 \\
2,060 \\
5,330 \\
2,700 \\
3,320 \\
3,970 \\
4,930 \\
4,980 \\
1,250 \\
1,550 \\
5,050\end{array}$ \\
\hline
\end{tabular}

\footnotetext{
Present datum.
} 


\section{Waccasassa River Basin}

(102) Waccasassa R1ver near Otter Creek, Fla.

Location.--Lat $29^{\circ} 22^{\prime}$, long $82^{\circ} 44^{\prime}$, in sec. 17, T. $13 \mathrm{~S} ., \mathrm{R}$. $16 \mathrm{E}$, near right bank at upstres s1de of bridge on State H1ghway $24,2.8$ miles northeast of village of otter Creek, and 11 mlles upstream from Otter creek. Records ava1lable.--U. S. Geological Survey: May 1945 to November 1953, da1ly gage heights and
discharges for main channel only.

Gage.--Water-stage recorder. Datum of gage $1 \mathrm{~s} 23.52 \mathrm{ft}$ above mean sea level, datum of 1929.

Stage-discharge relation.--Defined by current-meter measurements below 1,200 cfs for main channel only. Considerable shifting at all stages.

Remarks.--Waccasassa R1ver and Otter Creek are connected above State Highway 24 by swamps and numerous cross channels.

Annual peak stages and discharges

\begin{tabular}{c|c|c|c|c|c|c|c}
\hline $\begin{array}{c}\text { Calendar } \\
\text { year }\end{array}$ & Date & $\begin{array}{c}\text { Gage } \\
\text { height } \\
\text { (feet) }\end{array}$ & $\begin{array}{c}\text { Discharge } \\
\text { (cfs) }\end{array}$ & $\begin{array}{c}\text { Calendar } \\
\text { year }\end{array}$ & Date & $\begin{array}{c}\text { Gage } \\
\text { height } \\
\text { (feet) }\end{array}$ & $\begin{array}{c}\text { Discharge } \\
\text { (cfs) }\end{array}$ \\
\hline 1945 & Aug. 20 & 5.57 & 506 & 1950 & Sept. 7 & 7.16 & 1,210 \\
1946 & July 30 & 4.72 & 255 & 1951 & Nov. 17 & 5.00 & 354 \\
1947 & Sept. 25, 26 & 5.21 & 399 & 1952 & Feb. 26 & 5.10 & 376 \\
1948 & Mar. 12 & 6.39 & 902 & 1953 & Aug. 25 & 5.60 & 577 \\
1949 & Aug. 13 & 5.48 & 490 & & & & \\
\hline
\end{tabular}

(103) Otter Creek at Otter Creek, Fla.

Location.--Lat $29^{\circ} 19^{\prime}$, long $82^{\circ} 46^{\prime}$, in sec. 26, T. $13 \mathrm{~S} .$, R. 15 E., near right bank at upstream side of bridge on State Highway $24,0.5$ mile southwest of village of otter Creek and about 7 miles upstream from mouth.

Records ava1lable.--U. S. Geological Survey: May 1945 to November 1953, da1ly gage heights and discharges.

Gage.--Water-stage recorder. Datum of gage $1 \mathrm{~s} 13.35 \mathrm{ft}$ above mean sea level, datum of 1929. Stage-discharge relation.--Defined by current-meter measurements below 3,000 cfs.

Remarks.--Records include flow in main channel and a relief channel 0.4 mile east of gage. Waccasassa R1ver and otter Creek are connected above State H1ghway 24 by swamps and numerous cross channels.

Annual peak stages and discharges

\begin{tabular}{|c|c|c|c|c|c|c|c|}
\hline $\begin{array}{c}\text { Calendar } \\
\text { year }\end{array}$ & Date & $\begin{array}{c}\text { Gage } \\
\text { height } \\
\text { (feet) }\end{array}$ & $\begin{array}{c}\text { Discharge } \\
\text { (cfs) }\end{array}$ & $\begin{array}{c}\text { Calendar } \\
\text { year }\end{array}$ & Date & $\begin{array}{c}\text { Gage } \\
\text { height } \\
\text { (feet) }\end{array}$ & $\begin{array}{l}\text { Discharge } \\
\text { (cis) }\end{array}$ \\
\hline 1945 & Aug. 20 & 5.91 & 560 & 1950 & Sept. 6 & 7.93 & 3,000 \\
\hline $\begin{array}{l}1946 \\
1947 \\
1948 \\
1949\end{array}$ & $\begin{array}{l}\text { July } 31 \\
\text { Oct. } 17 \\
\text { Mar. } 12 \\
\text { Aug. } 14\end{array}$ & $\begin{array}{l}4.99 \\
5.38 \\
6.18 \\
5.60\end{array}$ & $\begin{array}{r}322 \\
542 \\
1,350 \\
788\end{array}$ & $\begin{array}{l}1951 \\
1952 \\
1953\end{array}$ & $\begin{array}{lr}\text { Nov. } & 17 \\
\text { Feb. } & 17 \\
\text { Aug. } & 9\end{array}$ & $\begin{array}{l}5.08 \\
5.05 \\
5.00\end{array}$ & $\begin{array}{l}509 \\
496 \\
474\end{array}$ \\
\hline
\end{tabular}




\section{Suwannee R1ver Basin}

(104) Suwannee River at Fargo, Ga.

Location.--Lat $30^{\circ} 41^{\prime}$, long $82^{\circ} 34^{\prime}$, on downstream side of Southern Ra1lway bridge at Fargo, Clinch County, 4 miles upstream from Suwanoochee Creek and 12 miles downstream from Mixons Ferry dam site.

Drainage area.--About 1,260 $\mathrm{sq} \mathrm{m} 1$ (1ncludes part of watershed in Okefenokee Swamp wh1ch 1s Indeterminate).

Records ava1lable.--U. S. Geological Survey: January 1927 to December 1931, Apr11 1937 to December 1953, da1ly gage helghts and discharges.

Gage.--Water-stage recorder. Datum of gage is $91.90 \mathrm{ft}$ above mean sea level, datum of 1929, supplementary adjustment of 1936. January 1927 to December 1931 (1ncomplete) and April 1937 to June 10, 1938, staff gage at site 1,000 ft upstream at datum $1.00 \mathrm{ft}$ higher. June 11 , 1938 , to Nov. 26, 1952, staff gage at site 1,000 $\mathrm{ft}^{\prime}$ upstream at present datum. (All gage readings listed below have been reduced to present datum).

Stage-discharge relation.--Defined by current-meter measurements below 12,000 cfs; affected by backwater.

Annual peak stages and discharges

\begin{tabular}{|c|c|c|c|c|c|c|c|}
\hline $\begin{array}{c}\text { Calendar } \\
\text { year }\end{array}$ & Date & $\begin{array}{l}\text { Gage } \\
\text { height } \\
\text { (feet) }\end{array}$ & $\begin{array}{l}\text { Discharge } \\
\text { (cfs) }\end{array}$ & $\begin{array}{l}\text { Calendar } \\
\text { year }\end{array}$ & Date & $\begin{array}{l}\text { Gage } \\
\text { height } \\
\text { (feet) }\end{array}$ & $\begin{array}{l}\text { Discharge } \\
\text { (cfs) }\end{array}$ \\
\hline $\begin{array}{l}1928 \\
1929\end{array}$ & $\begin{array}{ll}\text { Oct. } & 1 \\
\text { Oct. } & 3\end{array}$ & $\begin{array}{l}19.5 \\
19.6\end{array}$ & $\begin{array}{l}13,800 \\
11,900\end{array}$ & 1945 & Aug. 22 & 17.3 & 9,690 \\
\hline 1930 & oct. 3 & 11.4 & 2,460 & $\begin{array}{l}1946 \\
1947\end{array}$ & $\begin{array}{l}\text { Aug. } \\
\text { Oct. }\end{array}$ & $\begin{array}{l}14.2 \\
19.6\end{array}$ & $\begin{array}{r}5,920 \\
11,900\end{array}$ \\
\hline $\begin{array}{l}1938 \\
1939 \\
1940\end{array}$ & $\begin{array}{l}\text { Aug. } 8,9 \\
\text { Aug. } 29 \\
\text { Feb. } 21\end{array}$ & $\begin{array}{r}8.2 \\
11.6 \\
11.0\end{array}$ & $\begin{array}{l}1,300 \\
2,490 \\
2,060\end{array}$ & $\begin{array}{l}1948 \\
1949 \\
1950\end{array}$ & $\begin{array}{l}\text { Apr. } 4,5 \\
\text { Sept. } 10 \\
\text { Oct. } 26-30\end{array}$ & $\begin{array}{l}19.1 \\
14.3 \\
11.9\end{array}$ & $\begin{array}{r}11,000 \\
5,900 \\
3,260\end{array}$ \\
\hline $\begin{array}{l}1941 \\
1942 \\
1943 \\
1944 \\
\end{array}$ & $\begin{array}{l}\text { Nov. }{ }^{8-12} \\
\text { Jan. } 11 \\
\text { Mar. } 7 \\
\text { Aug. } 14-18\end{array}$ & $\begin{array}{r}7.8 \\
15.9 \\
3.8 \\
12.8\end{array}$ & $\begin{array}{r}870 \\
7,960 \\
258 \\
4,280\end{array}$ & $\begin{array}{l}1951 \\
1952 \\
1953\end{array}$ & $\begin{array}{lr}\text { Dec. } & 31 \\
\text { Mar. } & 4 \\
\text { Oct. } & 6\end{array}$ & $\begin{array}{l}12.8 \\
11.0 \\
14.6\end{array}$ & $\begin{array}{l}4,300 \\
2,390 \\
5,640\end{array}$ \\
\hline
\end{tabular}

(105) Suwannee R1ver at White Springs, Fla.

Location.--Lat $30^{\circ} 20^{\prime}$, long $82^{\circ} 44^{\prime}$, in sec. 8, T. 2 S., R. 16 E., on left bank at downstream s1de of bridge on U. S. H1ghway 4l, 1 mile southeast of White Springs.

Drainage area.--1,990 sq $\mathrm{ml}$ (Includes part of watershed in Okefenokee Swamp which 1s Indeterminate).

Records avallable.--U. S. Geological Survey: May 1906 to December 1908, February 1927 to December 1953, daliy gage helghts and discharges.

Gage.--Water-stage recorder. Datum of gage is $48.54 \mathrm{ft}$ above mean sea level (Corps of Engineers benchmark). May 28, 1906, to Dec. 31, 1908, cha1n gage, and Feb. 8, 1927, to July 31, 1932, staff gage at site 1 mile downstream at same datum.

Stage-discharge relation.--Defined by current-meter measurements below 28,000 cfs.

Historical data.--The flood of Apr. 5, 1948 was reported to be the highest known to residents of the area (since 1862). Flood of 1928 was reported to be highest known at that time. 
Suwannee R1ver Basin

(105) Suwannee River at White Springs, Fla.--Continued

Annual peak stages and discharges

\begin{tabular}{|c|c|c|c|c|c|c|c|}
\hline $\begin{array}{c}\text { Calendar } \\
\text { year }\end{array}$ & Date & $\begin{array}{l}\text { Gage } \\
\text { height } \\
\text { (feet) }\end{array}$ & $\begin{array}{c}\text { Discharge } \\
\text { (cfs) }\end{array}$ & $\begin{array}{c}\text { Calendar } \\
\text { year }\end{array}$ & Date & $\begin{array}{l}\text { Gage } \\
\text { height } \\
\text { (feet) }\end{array}$ & $\begin{array}{c}\text { Discharge } \\
\text { (cis) }\end{array}$ \\
\hline $\begin{array}{l}1906 \\
1907 \\
1908\end{array}$ & $\begin{array}{ll}\text { July } & 16 \\
\text { Oct. } & 4 \\
\text { Jan. } & 1,2\end{array}$ & $\begin{array}{l}23.2 \\
13.0 \\
20.9\end{array}$ & $\begin{array}{l}7,400 \\
3,540 \\
6,520\end{array}$ & $\begin{array}{l}1938 \\
1939 \\
1940\end{array}$ & $\begin{array}{lr}\text { Aug. } & 8 \\
\text { Aug. } & 28 \\
\text { Feb. } & 20\end{array}$ & $\begin{array}{l}15.23 \\
19.01 \\
17.75\end{array}$ & $\begin{array}{l}4,150 \\
5,640 \\
5,180\end{array}$ \\
\hline $\begin{array}{l}1927 \\
1928 \\
1929 \\
1930\end{array}$ & $\begin{array}{ll}\text { July } & 27 \\
\text { Sept. } & 30, \\
\text { Oct. } & 1 \\
\text { Oct. } & 5 \\
\text { Mar. } & 30,31, \\
\text { Apr. } & 1\end{array}$ & $\begin{array}{l}9.8 \\
33.9 \\
33.5 \\
30.4\end{array}$ & $\begin{array}{r}2,610 \\
20,600 \\
19,600 \\
12,600\end{array}$ & $\begin{array}{l}1941 \\
1942 \\
1943 \\
1944 \\
1945\end{array}$ & $\begin{array}{lr}\text { Dec. } & 29 \\
\text { Jan. } & 15 \\
\text { Mar. } & 8 \\
\text { Aug. } & 11,12 \\
\text { Aug. } & 26\end{array}$ & $\begin{array}{r}14.90 \\
30.22 \\
4.74 \\
24.37 \\
33.20\end{array}$ & $\begin{array}{r}4,080 \\
11,700 \\
594 \\
7,690 \\
16,500\end{array}$ \\
\hline $\begin{array}{l}1931 \\
1932 \\
1933 \\
1934 \\
1935\end{array}$ & $\begin{array}{l}\text { Jan. } \frac{24,25}{22} \\
\text { Sept. } 22 \\
\text { Feb. } 17 \\
\text { Aug. } 13 \\
\text { Sept. } 22\end{array}$ & $\begin{array}{l}15.0 \\
29.26 \\
28.97 \\
10.10 \\
21.07\end{array}$ & $\begin{array}{r}4,280 \\
10,100 \\
10,200 \\
2,220 \\
6,330\end{array}$ & $\begin{array}{l}1946 \\
1947 \\
1948 \\
1949 \\
1950\end{array}$ & $\begin{array}{lr}\text { Aug. } & 11 \\
\text { Oct. } & 29 \\
\text { Apr. } & 5 \\
\text { Sept. } & 12 \\
\text { Oct. } & 30\end{array}$ & $\begin{array}{l}26.11 \\
35.47 \\
36.65 \\
24.91 \\
15.16\end{array}$ & $\begin{array}{r}8,340 \\
23,700 \\
28,500 \\
7,880 \\
4,200\end{array}$ \\
\hline $\begin{array}{l}1936 \\
1937 \\
\end{array}$ & $\begin{array}{ll} & \\
\text { Feb. } & 24 \\
\text { Apr. } & 18 \\
\end{array}$ & $\begin{array}{l}16.00 \\
29.54 \\
\end{array}$ & $\begin{array}{r}4,480 \\
11,100 \\
\end{array}$ & $\begin{array}{l}1951 \\
1952 \\
1953 \\
\end{array}$ & $\begin{array}{lr}\text { Nov. } & 17 \\
\text { Jan. } & 5 \\
\text { Oct. } & 10 \\
\end{array}$ & $\begin{array}{l}19.29 \\
19.34 \\
28.36 \\
\end{array}$ & $\begin{array}{r}5,760 \\
5,780 \\
10,100 \\
\end{array}$ \\
\hline
\end{tabular}

(106) Alapaha R1ver near Alapaha, Ga.

Location.--Lat $31^{\circ} 23^{\prime}$, long $83^{\circ} 10^{\prime}$, on downstream side of bridge on State H1ghway $50,2 \mathrm{~m} 11 \mathrm{es}$ east of Alapaha, Berrien County, and 6 miles upstream from W1llacoochee R1ver.

Drainage area. $--644 \mathrm{sq} \mathrm{ml}$.

Records ava1lable.--U. S. Geological Survey: Apr1l 1937 to December 1953, da1ly gage helghts and discharges.

Gage.--Water-stage recorder. Datum of gage 1 s $209.34 \mathrm{ft}$ above mean sea level, datum of 1929. Prior to Sept. 8, 1943, staff gage at same site and datum.

Stage-discharge relation.--Defined by current-meter measurements below $12,000 \mathrm{cfs}$.

Historical data.--Flood of 1928 reached a stage of $18.0 \mathrm{ft}$ from information by Georgia H1ghway Department.

Annual peak stages and discharges

\begin{tabular}{|c|c|c|c|c|c|c|c|}
\hline $\begin{array}{c}\text { Calendar } \\
\text { year }\end{array}$ & Date & $\begin{array}{c}\text { Gage } \\
\text { height } \\
\text { (feet) }\end{array}$ & $\begin{array}{c}\text { Discharge } \\
\text { (cfs) }\end{array}$ & $\begin{array}{c}\text { Calendar } \\
\text { year }\end{array}$ & Date & $\begin{array}{c}\text { Gage } \\
\text { height } \\
\text { (feet) }\end{array}$ & $\begin{array}{c}\text { Discharge } \\
\text { (cfs) }\end{array}$ \\
\hline 1928 & - & 18.0 & 16,000 & 1946 & Jan. 23 & 11.6 & $\begin{array}{l}3,040 \\
6,160\end{array}$ \\
\hline $\begin{array}{l}1938 \\
1939 \\
1940\end{array}$ & $\begin{array}{l}\text { Apr. } 15 \\
\text { Mar. } 3 \\
\text { Feb. } 25\end{array}$ & $\begin{array}{r}8.6 \\
15.3 \\
10.8\end{array}$ & $\begin{array}{l}1,060 \\
9,220 \\
2,290\end{array}$ & $\begin{array}{l}1948 \\
1949 \\
1950\end{array}$ & $\begin{array}{l}\text { Apr: } 4 \\
\text { Feb. } 12 \\
\text { Mar. } 22\end{array}$ & $\begin{array}{r}16.8 \\
11.5 \\
8.9\end{array}$ & $\begin{array}{r}12,700 \\
2,920 \\
1,130\end{array}$ \\
\hline $\begin{array}{l}1941 \\
1942 \\
1943 \\
1944 \\
1945\end{array}$ & $\begin{array}{l}\text { Mar. } 25,26 \\
\text { Jan. } 9 \\
\text { Mar. } 26,27 \\
\text { Apr. } 21 \\
\text { July } 22\end{array}$ & $\begin{array}{r}9.5 \\
12.9 \\
11.3 \\
15.0 \\
11.5\end{array}$ & $\begin{array}{l}1,450 \\
4,960 \\
2,700 \\
8,620 \\
2,920\end{array}$ & $\begin{array}{l}1951 \\
1952 \\
1953\end{array}$ & $\begin{array}{l}\text { Dec. } 25,26 \\
\text { Feb. } 24 \\
\text { Oct. } 6\end{array}$ & $\begin{array}{l}10.9 \\
10.8 \\
10.9\end{array}$ & $\begin{array}{l}2,300 \\
2,220 \\
2,300\end{array}$ \\
\hline
\end{tabular}


Suwannee River Basin

(107) Alapaha River at Statenville, Ga.

Location.--Lat $30^{\circ} 40^{\prime}$, long $83^{\circ} \mathrm{O} 1^{\prime}$, at downstream side of left bank pier of bridge on State Highway 94 , a quarter of a mile west of Statenville, Echols County.

Dra1nage area. $--1,400 \mathrm{sq} \mathrm{ml}$, approximately.

Records ava1lable.--U. S. Geological Survey: December 1931 to December 1953, da1ly gage helghts and discharges.

Gage.--Water-stage recorder. Datum of gage is $76.77 \mathrm{ft}$ above mean sea level (levels by Georgla State Highway Department). Dec. 10, 1931, to July 9, 1935, chain gage, July 10, 1935, to Nov. 30,1949 , staff gage at site $200 \mathrm{ft}$ upstream, Dec. 1, 1949, to Nov. 22, 1952, w1rewelght gage at present datum.

Stage-discharge relation.--Defined by current-meter measurements throughout range in stage.

Historical data.--Flood of May 1, 1928, reached a stage of $28.5 \mathrm{ft}$, from information furnished by local resident. Flood of April 1948 was the highest since 1862, from information furnished by a local resident.

Remarks.--Records for perlod from October 1928 to September 1931, obtained at Mayday, Georgia, $11 \mathrm{~m} i l e s$ upstream from present site. Drainage area $1,300 \mathrm{sq} \mathrm{m}$, approximately.

Annual peak stages and discharges

\begin{tabular}{|c|c|c|c|c|c|c|c|}
\hline $\begin{array}{c}\text { Calendar } \\
\text { year }\end{array}$ & Date & $\begin{array}{l}\text { Gage } \\
\text { height } \\
\text { (feet) }\end{array}$ & $\begin{array}{l}\text { Discharge } \\
\text { (cfs) }\end{array}$ & $\begin{array}{c}\text { Calendar } \\
\text { year }\end{array}$ & Date & $\begin{array}{c}\text { Gage } \\
\text { height } \\
\text { (feet) }\end{array}$ & $\begin{array}{l}\text { Discharge } \\
\text { (cfs) }\end{array}$ \\
\hline 1928 & May & 28.5 & 17,400 & \multirow{5}{*}{$\begin{array}{l}1941 \\
1942 \\
1943 \\
1944 \\
1945\end{array}$} & \multirow{4}{*}{$\begin{array}{ll}\text { Mar. } & 31 \\
\text { Mar. } & 23 \\
\text { Apr. } & 3,4 \\
\text { Apr. } & 27 \\
\text { Aug. } & 25\end{array}$} & \multirow{4}{*}{$\begin{array}{r}9.4 \\
22.3 \\
12.2 \\
26.8 \\
22.8\end{array}$} & \multirow{4}{*}{$\begin{array}{r}1,910 \\
6,370 \\
2,700 \\
11,100 \\
6,630\end{array}$} \\
\hline 1929 & Mar. 22 & - & 14,200 & & & & \\
\hline 1930 & Feb. 8 & - & 4,730 & & & & \\
\hline 1931 & May & _ & 3,370 & & & & \\
\hline 1932 & Aug. & 11.6 & $\frac{2,740}{2,740}$ & & & & \\
\hline 1933 & Feb. & 21.8 & 6,140 & 1946 & Jan. & 17.2 & 4,160 \\
\hline 1934 & June & 10.8 & 2,420 & 1947 & Apr. & 22.4 & 5,990 \\
\hline 1935 & Sept. 6,8 & 14.6 & 3,440 & 1948 & $\mathrm{Apr}$. & 29.8 & 27,300 \\
\hline 1936 & Feb $\quad 19$ & 185 & 4,900 & $\begin{array}{l}1949 \\
1950\end{array}$ & $\begin{array}{ll}\text { Sept. } & 5 \\
\text { Anr } & \end{array}$ & $\begin{array}{r}18.5 \\
8.7\end{array}$ & 4,620 \\
\hline 1937 & Apr. & 22.8 & 6.560 & & & & 1,140 \\
\hline 1938 & Sept. 30 & 6.4 & 1,130 & 1951 & Apr. & 14.9 & 3,480 \\
\hline 1939 & Mar. & 26.4 & 10,500 & 1952 & Mar. 13 & 14.5 & 3,360 \\
\hline 1940 & Feb. 28,29 & 11.7 & 2,630 & 1953 & Oct. & 16.9 & 4,100 \\
\hline
\end{tabular}

(108) Little River near Adel, Ga.

Location.--Lat $31^{\circ} 09^{\prime}$, long $83^{\circ} 33^{\prime}$, on right bank $500 \mathrm{ft}$ downstream from bridge on State Highway 37, half a mile downstream from Georgla and Florida Rallroad bridge, $5 \frac{1}{2}$ miles upstream from Bear Creek, 6 miles downstream from Warrior Creek, and 7 miles west of Adel, Cook County.

Dra1nage area. $--547 \mathrm{sq} \mathrm{ml}$.

Records avallable.--U. S. Geological Survey: June 1940 to December 1953, da1ly gage helghts and discharges.

Gage.--Water-stage recorder. Datum of gage is $171.08 \mathrm{ft}$ above mean sea level, datum of 1929.

Stage-discharge relation.--Defined by current-meter measurements below 13,000 cfs and by contracted-opening determination for peak flow of Apr. 2, 1948.

H1storical data.--The flood of August 1928 reached a stage of $20.5 \mathrm{ft}$, from information by Georgla H1ghway Department.

The flood of April 1948 was the highest known to local residents. 
Suwannee R1ver Basin

(108) Little River near Adel, Ga.--Continued

Annual peak stages and discharges

\begin{tabular}{|c|c|c|c|c|c|c|c|}
\hline $\begin{array}{c}\text { Calendar } \\
\text { year }\end{array}$ & Date & $\begin{array}{c}\text { Gage } \\
\text { height } \\
\text { (feet) }\end{array}$ & $\begin{array}{c}\text { Discharge } \\
\text { (cfs) }\end{array}$ & $\begin{array}{c}\text { Calendar } \\
\text { year }\end{array}$ & Date & $\begin{array}{c}\text { Gage } \\
\text { height } \\
\text { (feet) }\end{array}$ & $\begin{array}{c}\text { Dischara } \\
\text { (cfs) }\end{array}$ \\
\hline $\begin{array}{l}1928 \\
1941 \\
1942 \\
1943 \\
1944 \\
1945 \\
1946\end{array}$ & $\begin{array}{lr}\text { Aug. }- \\
\text { Mar. } & 24 \\
\text { Jan. } & 6 \\
\text { May } & 26 \\
\text { Mar. } & 8 \\
\text { July } & 22 \\
& \\
\text { Jan. } & 25\end{array}$ & $\begin{array}{l}20.5 \\
11.5 \\
16.7 \\
15.6 \\
18.7 \\
15.3 \\
14.6\end{array}$ & $\begin{array}{r}32,500 \\
1,520 \\
5,900 \\
3,950 \\
16,700 \\
3,670 \\
2,920\end{array}$ & $\begin{array}{l}1947 \\
1948 \\
1949 \\
1950 \\
1951 \\
1952 \\
1953\end{array}$ & $\begin{array}{lr}\text { Mar. } & 10 \\
\text { Apr. } & 2 \\
\text { Apr. } & 14 \\
\text { July } & 15 \\
& \\
\text { Dec. } & 25 \\
\text { Mar. } & 27 \\
\text { Sept. } & 30\end{array}$ & $\begin{array}{l}17.1 \\
21.0 \\
14.8 \\
14.4 \\
15.8 \\
14.7 \\
14.9\end{array}$ & $\begin{array}{r}7,580 \\
38,800 \\
3,210 \\
2,750 \\
4,360 \\
3,010 \\
3,210\end{array}$ \\
\hline
\end{tabular}

(109) W1thlacoochee River near Quitman, Ga.

Location.--Lat $30^{\circ} 47^{\prime}$, long $83^{\circ} 27^{\prime}$, at bridge on U. S. Highway 84 , 500 ft upstream from T1ger Creek, $800 \mathrm{ft}$ downstream from Atlant1c Coast Line Ra1lroad bridge, a quarter of a mile east of Blue Springs, and 6 miles east of Quitman, Brooks County.

Drainage area. $--1,560 \mathrm{sq} \mathrm{m} 1$, approximately.

Records avallable.--U. S. Geological Survey: October 1920 to March 1921, September 1928 to December 1931, June 1937 to September 1948, da1ly gage he1ghts and discharges. S1nce January 1953, annual flood peaks.

Gage.--Non-recording gage. Datum of gage $1884.30 \mathrm{ft}$ above mean sea level, datum of 1929 , supplementary adfustment of 1936. Gage used 1928-31 was at datum 5:0 ft lower. Since September 1948, crest-stage gage (too high to ind1cate annual flood peaks prior to January 1953). All gage helghts tabulated below have been reduced to present datum for this report.

Stage-d1scharge relation.--Defined by current-meter measurements below 50,000 cfs.

H1storical data.--At the time of the August 1928 flood, 1t was reported to be the highest known to the oldest residents of the area.

Annual peak stages and discharges

\begin{tabular}{|c|c|c|c|c|c|c|c|}
\hline $\begin{array}{c}\text { Calendar } \\
\text { year }\end{array}$ & Date & $\begin{array}{l}\text { Gage } \\
\text { height } \\
\text { (feet) }\end{array}$ & $\begin{array}{c}\text { Discharge } \\
\text { (cfs) }\end{array}$ & $\begin{array}{c}\text { Calendar } \\
\text { year }\end{array}$ & Date & $\begin{array}{l}\text { Gage } \\
\text { height } \\
\text { (feet) }\end{array}$ & $\begin{array}{c}\text { Discharge } \\
\text { (cfs) }\end{array}$ \\
\hline $\begin{array}{l}1928 \\
1929 \\
1930 \\
1931\end{array}$ & $\begin{array}{l}\text { Aug. } 19 \\
\text { Mar. } 20 \\
\text { Feb. } 12 \\
\text { Aug. } 21\end{array}$ & $\begin{array}{l}31.3 \\
24.8 \\
21.6 \\
9.6\end{array}$ & $\begin{array}{r}62,000 \\
18,000 \\
6,250 \\
2,760\end{array}$ & $\begin{array}{l}1942 \\
1943 \\
1944 \\
1945\end{array}$ & $\begin{array}{lr}\text { Jan. } & 9 \\
\text { Mar. } 11 \\
\text { Mar. } 11 \\
\text { July } 27\end{array}$ & $\begin{array}{l}22.0 \\
12.5 \\
25.5 \\
21.4\end{array}$ & $\begin{array}{r}11,600 \\
3,730 \\
21,000 \\
10,600\end{array}$ \\
\hline $\begin{array}{l}1938 \\
1939 \\
1940\end{array}$ & $\begin{array}{lr}\text { Apr. } & 17 \\
\text { Mar: } & 5 \\
\text { Feb. } 25\end{array}$ & $\begin{array}{l}5.13 \\
22.6 \\
11.0\end{array}$ & $\begin{array}{r}955 \\
12,800 \\
3,450\end{array}$ & $\begin{array}{l}1946 \\
1947 \\
1948 \\
1949\end{array}$ & $\begin{array}{lr}\text { Mar. } & 21 \\
\text { Dec. } & 17 \\
\text { Apr. } & 4 \\
\text { Apr. } & 19\end{array}$ & $\begin{array}{l}18.2 \\
25.5 \\
31.7 \\
16.6\end{array}$ & $\begin{array}{r}6,860 \\
21,000 \\
66,000 \\
5,660\end{array}$ \\
\hline 1941 & Mar. 29 & 8.3 & 2,320 & 1953 & $\operatorname{Oct} .1$ & 18.0 & 6,700 \\
\hline
\end{tabular}




\section{Suwannee R1ver Bas1n}

(110) Withlacoochee River near Pinetta, Fla.

Location.--Lat $30^{\circ} 36^{\prime}$, long $83^{\circ} 16^{\prime}$, on line between secs. 6 and $7, T$. 2 N., R. 11 E., on right bank $30 \mathrm{ft}$ downstream from highway bridge a quarter of a mile west of Bellville, and $5 \mathrm{miles}$ east of P1netta.

Drainage area.--2,220 sq $\mathrm{ml}$, approximately.

Records avallable.--U. S. Geological Survey: December 1931 to December 1953, da1ly gage helghts and discharges.

Gage.--Water-stage recorder. Datum of gage $1 \mathrm{~s} 47.21 \mathrm{ft}$ above mean sea level (levels by Corps of Englneers). Prior to Jan. 27, 1939, cha1n gage and Jan. 27, 1939, to Dec. 2, 1941, w1rewelght gage at same site and datum.

Stage-discharge relation.--Deflned by current-meter measurements below 58,000 cfs. Sl1ght shifting at medium and low stages.

H1storical data.--Flood of Apr. 5, 1948, is the maximum known. Flood of August 1928 reached a stage of $36.75 \mathrm{ft}$, from floodmarks, and was the h1ghest known at that time, according to local residents.

Annual peak stages and discharges

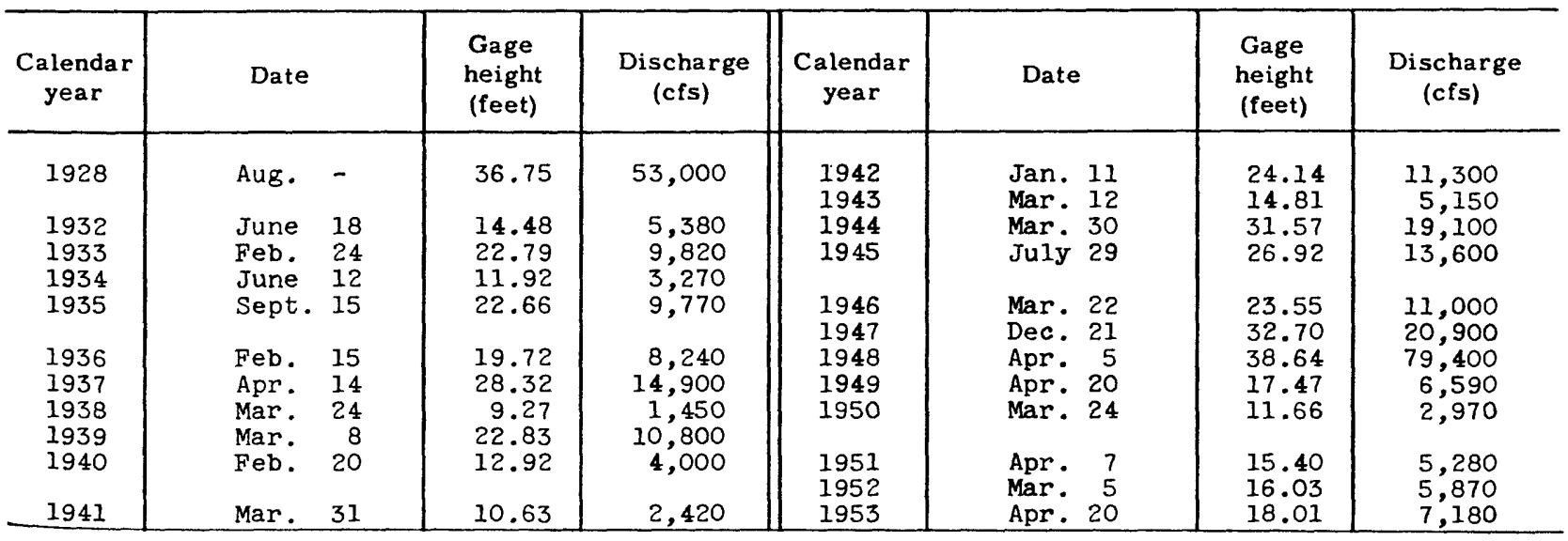

(111) Suwannee River at Ellaville, Fla.

Location.--Lat $30^{\circ} 23^{\prime}$, long $83^{\circ} 10^{\prime}$, in sec. 24, T. I S., R. 11 E., on left bank at Ellaville, $200 \mathrm{ft}$ upstream from Seaboard Air Line Ra1lroad bridge, 200 f'́ downstream from Withlacoochee River, and a quarter of a mile upstream from bridge on U. S. Highway 90 .

Drainage area. $--6,580 \mathrm{sq} \mathrm{ml}$, approximately.

Records ava1lable.--U. S. Geologlcal Survey: January 1927 to December 1953, da1ly gage he1ghts and discharges.

Gage.--Water-stage recorder. Datum of gage $1 \mathrm{~s} 27.82 \mathrm{ft}$ above mean sea level, (levels by Corps of Eng1neers). Prior to June 20, 1932, staff gage at same site and datum.

Stage-discharge relation.--Defined by current-meter measurements.

H1storical data.--Flood of Apr. 7, 8, 1948, is the maximum known. 
Suwannee River Basin

(111) Suwannee River at Ellaville, Fla.--Continued

Annual peak stages and discharges

\begin{tabular}{|c|c|c|c|c|c|c|c|c|}
\hline $\begin{array}{c}\text { Calendar } \\
\text { year }\end{array}$ & Date & & $\begin{array}{l}\text { Gage } \\
\text { height } \\
\text { (feet) }\end{array}$ & $\begin{array}{c}\text { Discharge } \\
\text { (cfs) }\end{array}$ & $\begin{array}{c}\text { Calendar } \\
\text { year }\end{array}$ & Date & $\begin{array}{l}\text { Gage } \\
\text { height } \\
\text { (feet) }\end{array}$ & $\begin{array}{c}\text { Dischara } \\
\text { (cfs) }\end{array}$ \\
\hline $\begin{array}{l}1927 \\
1928 \\
1929 \\
1930\end{array}$ & $\begin{array}{ll}\text { Aug. } & 28 \\
\text { Aug. } & 20 \\
\text { Oct. } & 12, \\
\text { Apr. } & 3,\end{array}$ & $\begin{array}{l}13 \\
4\end{array}$ & $\begin{array}{l}6.0 \\
37.1 \\
26.9 \\
24.28\end{array}$ & $\begin{array}{r}5,900 \\
73,000 \\
34,000 \\
29,200\end{array}$ & $\begin{array}{l}1941 \\
1942 \\
1943 \\
1944 \\
1945\end{array}$ & $\begin{array}{lr}\text { July } & 29 \\
\text { Mar. } & 26 \\
\text { Mar. } & 14 \\
\text { Apr. } & 9 \\
\text { Aug. } & 28\end{array}$ & $\begin{array}{r}6.24 \\
24.90 \\
7.43 \\
23.53 \\
24.26\end{array}$ & $\begin{array}{r}5,710 \\
27,200 \\
6,790 \\
25,000 \\
26,200\end{array}$ \\
\hline $\begin{array}{l}1931 \\
1932 \\
1933 \\
1934 \\
1935\end{array}$ & $\begin{array}{ll}\text { Jan. } 25, \\
\text { Sept. } 27 \\
\text { Feb. } 25, \\
\text { June } 16 \\
\text { Sept. } 18\end{array}$ & $\begin{array}{l}26 \\
26\end{array}$ & $\begin{array}{c}10.70 \\
15.67 \\
24.02 \\
6.6 \\
18.35\end{array}$ & $\begin{array}{r}9,730 \\
15,500 \\
26,400 \\
6,200 \\
18,300\end{array}$ & $\begin{array}{l}1946 \\
1947 \\
1948 \\
1949 \\
1950\end{array}$ & $\begin{array}{ll}\text { Mar. } & 23 \\
\text { Nov. } & 22,23 \\
\text { Apr. } & 7,8 \\
\text { Sept. } & 11 \\
\text { Apr. } & 7\end{array}$ & $\begin{array}{r}17.71 \\
30.57 \\
40.88 \\
16.10 \\
6.82\end{array}$ & $\begin{array}{r}17,500 \\
39,800 \\
95,300 \\
15,700 \\
6,480\end{array}$ \\
\hline $\begin{array}{l}1936 \\
1937 \\
1938 \\
1939 \\
1940\end{array}$ & $\begin{array}{ll}\text { Feb. } & 27 \\
\text { Apr. } & 17 \\
\text { Aug. } & 13 \\
\text { Mar. } & 13 \\
\text { Feb. } & 28\end{array}$ & & $\begin{array}{r}15.16 \\
23.66 \\
5.61 \\
15.10 \\
10.59\end{array}$ & $\begin{array}{r}14,600 \\
25,200 \\
5,120 \\
14,500 \\
9,670 \\
\end{array}$ & $\begin{array}{l}1951 \\
1952 \\
1953\end{array}$ & $\begin{array}{ll}\text { Apr. } & 9 \\
\text { Mar. } & 7 \\
\text { Oct. } & 9\end{array}$ & $\begin{array}{l}10.96 \\
14.76 \\
18.80\end{array}$ & $\begin{array}{l}10,100 \\
14,300 \\
19,200\end{array}$ \\
\hline
\end{tabular}

(112) Suwannee River at Luraville, Fla.

Location.--Lat $30^{\circ} \mathrm{O} 6^{\prime}$, long $83^{\circ} 10^{\prime}$, in sec. 30, T. $4 \mathrm{~S} ., \mathrm{R}$. 12 E., near left bank on upstream side of county highway bridge, three miles upstream from Grants Ferry Shoals, and one mile south of Luravilie.

Drainage area. $--6,900 \mathrm{sq} \mathrm{ml}$, approximately.

Records ava1lable.--U. S. Geological Survey: February 1927 to December 1937, da1ly gage helghts and discharges.

Gage.--Staff gage read once da1ly. Datum of gage was $16.49 \mathrm{ft}$ above mean sea level (Corps of Engineers benchmark).

Stage-discharge relation.--Defined by current-meter measurements below 33,000 cfs.

H1storlcal data.--Maximum known flood of Apr11 1948 reached a stage of about 37.0 ft, from Information furnished by Corps of Engineers.

Annual peak stages and discharges

\begin{tabular}{|c|c|c|c|c|c|c|c|}
\hline $\begin{array}{c}\text { Calendar } \\
\text { year }\end{array}$ & Date & $\begin{array}{c}\text { Gage } \\
\text { height } \\
\text { (feet) }\end{array}$ & $\begin{array}{c}\text { Discharge } \\
\text { (cfs) }\end{array}$ & $\begin{array}{c}\text { Calendar } \\
\text { year }\end{array}$ & Date & $\begin{array}{l}\text { Gage } \\
\text { height } \\
\text { (feet) }\end{array}$ & $\begin{array}{c}\text { Discharge } \\
\text { (cfs) }\end{array}$ \\
\hline $\begin{array}{l}1927 \\
1928 \\
1929 \\
1930 \\
1931 \\
1932 \\
1933 \\
\end{array}$ & $\begin{array}{ll}\text { Aug. } & 29 \\
\text { Aug. } & 24 \\
\text { Mar. } & 29,30 \\
\text { Apr. } & 7-10 \\
& \\
\text { Jan. } & 27 \\
\text { Sept. } & 28-30 \\
\text { Mar. } & 1\end{array}$ & $\begin{array}{r}5.8 \\
33.7 \\
22.5 \\
21.6 \\
9.84 \\
13.80 \\
20.28\end{array}$ & $\begin{array}{r}6,090 \\
66,000 \\
27,200 \\
25,900 \\
9,810 \\
14,800 \\
24,000\end{array}$ & $\begin{array}{l}1934 \\
1935 \\
1936 \\
1937 \\
1948\end{array}$ & $\begin{array}{l}\text { June } 17 \\
\text { Sept. } 22,23 \\
\text { Feb. } 29, \\
\text { Mar. } \quad 1,2 \\
\text { Apr. } 19 \\
\text { Apr. } \quad-\end{array}$ & $\begin{array}{r}6.67 \\
15.80 \\
13.10 \\
19.70 \\
37.0\end{array}$ & $\begin{array}{r}6,560 \\
17,500 \\
13,900 \\
23,300 \\
-\end{array}$ \\
\hline
\end{tabular}


Suwannee River Basin

(113) Suwannee River at Branford, Fla.

Location.--Lat $29^{\circ} 57^{\prime}$, long $82^{\circ} 56^{\prime}$, in sec. 17 or 20 , T. 6 S., R. 14 E., near left bank on upstream side of bridge on U. S. Highways 27 and 129 at Branford, $10 \frac{3}{4}$ miles upstream from Santa Fe River.

Drainage area.--7,090 $\mathrm{sq} \mathrm{mi}$, approximately.

Records available.--U. S. Geological Survey: July 1931 to December 1953, da1ly gage heights and discharges.

Gage.--Wire-welght gage read once daily. Datum of gage is $4.81 \mathrm{ft}$ above mean sea level, datum of 1929. Prior to June 15, 1933, chain gage at same site and datum.

Stage-discharge relation.--Defined by current-meter measurements.

Historlcal data.--Flood of Aug. 26, 1928, reached a stage of $32.0 \mathrm{ft}$, from floodmarks.

Annual peak stages and discharges

\begin{tabular}{|c|c|c|c|c|c|c|c|}
\hline $\begin{array}{c}\text { Calendar } \\
\text { year }\end{array}$ & Date & $\begin{array}{l}\text { Gage } \\
\text { height } \\
\text { (feet) }\end{array}$ & $\begin{array}{l}\text { Discharge } \\
\text { (cfs) }\end{array}$ & $\begin{array}{c}\text { Calendar } \\
\text { year }\end{array}$ & Date & $\begin{array}{l}\text { Gage } \\
\text { height } \\
\text { (feet) }\end{array}$ & $\begin{array}{c}\text { Discharge } \\
\text { (cfs) }\end{array}$ \\
\hline 1928 & Aug. 26 & 32.0 & 61,100 & $\begin{array}{l}1942 \\
1943\end{array}$ & $\begin{array}{ll}\text { Mar. } & 28 \\
\text { Mar. } & 17\end{array}$ & $\begin{array}{r}23.70 \\
9.20 \\
97\end{array}$ & $\begin{array}{r}24,900 \\
6,730\end{array}$ \\
\hline $\begin{array}{l}1932 \\
1933 \\
1934\end{array}$ & $\begin{array}{lr}\text { Sept. } & 30 \\
\text { Mar. } & 1 \\
\text { June } & 20\end{array}$ & $\begin{array}{l}16.66 \\
21.96 \\
12.76\end{array}$ & $\begin{array}{r}14,800 \\
24,100 \\
8,350\end{array}$ & $\begin{array}{l}1944 \\
1945\end{array}$ & $\begin{array}{ll}\text { Apr. } & 11 \\
\text { Aug. } & 31\end{array}$ & $\begin{array}{l}21.42 \\
22.44\end{array}$ & $\begin{array}{l}21,700 \\
22,500\end{array}$ \\
\hline 1935 & Sept. 24 & 19.08 & 16,200 & $\begin{array}{l}1946 \\
1947\end{array}$ & $\begin{array}{ll}\text { Aug. } & 13 \\
\text { Nov. } & 24-26\end{array}$ & $\begin{array}{l}17.77 \\
26.95\end{array}$ & $\begin{array}{l}14,700 \\
32,500\end{array}$ \\
\hline $\begin{array}{l}1936 \\
1937 \\
1938 \\
1939\end{array}$ & $\begin{array}{ll}\text { Mar. } & 1 \\
\text { Apr: } & 20 \\
\text { Aug. } & 14,15 \\
\text { Mar. } & 15\end{array}$ & $\begin{array}{r}16.18 \\
21.65 \\
8.31 \\
14.48\end{array}$ & $\begin{array}{r}14,200 \\
22,700 \\
5,530 \\
13,300\end{array}$ & $\begin{array}{l}1948 \\
1949 \\
1950\end{array}$ & $\begin{array}{ll}\text { Apr. } & 11 \\
\text { Sept. } & 13 \\
\text { Oct. } & 28\end{array}$ & $\begin{array}{l}34.07 \\
18.11 \\
10.75\end{array}$ & $\begin{array}{r}83,900 \\
15,600 \\
7,180\end{array}$ \\
\hline 1940 & Feb. $\quad 29$ & 12.08 & & $\begin{array}{l}1951 \\
1952\end{array}$ & $\begin{array}{ll}\text { Apr. } & 11 \\
\text { Mar. } & 16-19\end{array}$ & $\begin{array}{l}12.19 \\
16.40\end{array}$ & $\begin{array}{r}9,480 \\
13,700\end{array}$ \\
\hline 1941 & $\begin{array}{ll}\text { Apr. } & 1,3 \\
\text { Aug. } & 1\end{array}$ & 8.86 & $\begin{array}{c}5,790 \\
- \\
\end{array}$ & 1953 & oct. 11 & 18.96 & 16,700 \\
\hline
\end{tabular}

(114) Santa Fe River at Worthington, Fla.

Location.--Lat $29^{\circ} 55^{\prime}$, long $82^{\circ} 26^{\prime}$, on line between secs. 32 and 33, T. 6 S., R. 19 E., near left bank on downstream side of bridge on State Highway 23, half a mile south of Worthington, and three-quarters of a mile downstream from New River.

Drainage area. $--630 \mathrm{sq} \mathrm{mi}$, approximately.

Records avallable.--U. S. Geological Survey: November 1931 to December 1953, da1ly gage helghts and discharges.

Gage.-Water-stage recorder. Datum of gage is $42.74 \mathrm{ft}$ above mean sea level (levels by Corps of Engineers). Prior to Jan. 16, 1939, staff gage at site a quarter of a mile downstream at same datum. Jan. 17, 1939, to July 23, 1953, w1re-welght gage read once dally at same site and datum.

Stage-discharge relation.--Defined by current-meter measurements; considerable shifting at all. stages. 
Suwannee River Basin

(114) Santa Fe River at Worthington, Fla.--Continued

Annual peak stages and discharges

\begin{tabular}{|c|c|c|c|c|c|c|c|}
\hline $\begin{array}{c}\text { Calendar } \\
\text { year }\end{array}$ & Date & $\begin{array}{l}\text { Gage } \\
\text { height } \\
\text { (feet) }\end{array}$ & $\begin{array}{c}\text { Discharge } \\
\text { (cfs) }\end{array}$ & $\begin{array}{c}\text { Calendar } \\
\text { year }\end{array}$ & Date & $\begin{array}{l}\text { Gage } \\
\text { height } \\
\text { (feet) }\end{array}$ & $\begin{array}{l}\text { Discharse } \\
\quad(c / s)\end{array}$ \\
\hline $\begin{array}{l}1932 \\
1933 \\
1934 \\
1935\end{array}$ & $\begin{array}{lr}\text { Sept. } & 17 \\
\text { Sept. } & 8 \\
\text { June } & 17 \\
\text { Sept. } \quad 8\end{array}$ & $\begin{array}{l}16.46 \\
18.20 \\
24.83 \\
22.37\end{array}$ & $\begin{array}{r}1,540 \\
3,120 \\
17,500 \\
11,200\end{array}$ & \multirow{3}{*}{$\begin{array}{l}1943 \\
1944 \\
1945 \\
1946 \\
1947 \\
1948 \\
1949 \\
1950\end{array}$} & $\begin{array}{ll}\text { Aug. } & 18 \\
\text { Oct. } & 21 \\
\text { Aug. } & 22\end{array}$ & \multirow{3}{*}{$\begin{array}{l}17.05 \\
24.94 \\
18.74 \\
19.1 \\
24.58 \\
24.60 \\
21.25 \\
24.03\end{array}$} & \multirow{3}{*}{$\begin{array}{r}2,160 \\
15,700 \\
4,000 \\
4,500 \\
14,900 \\
14,900 \\
7,570 \\
12,400\end{array}$} \\
\hline $\begin{array}{l}1936 \\
1937 \\
1938 \\
\end{array}$ & $\begin{array}{l}\text { Apr. } \\
\text { Sept. } 1 \\
\text { Oct. } 26 \\
\end{array}$ & $\begin{array}{l}16.90 \\
21.36 \\
21.07 \\
\end{array}$ & $\begin{array}{l}1,780 \\
8,780 \\
8,120 \\
\end{array}$ & & $\begin{array}{lr}\text { Aug. } & 2 \\
\text { Sept. } & 26 \\
\text { Mar. } & 12 \\
\text { Sept. } & 3\end{array}$ & & \\
\hline $\begin{array}{l}1939 \\
1940\end{array}$ & $\begin{array}{l}\text { Aug. } 10 \\
\text { Feb. } 22\end{array}$ & $\begin{array}{l}16.61 \\
16.62\end{array}$ & $\begin{array}{l}2,140 \\
1,950\end{array}$ & & Sept. & & \\
\hline $\begin{array}{l}1941 \\
1942 \\
\end{array}$ & $\begin{array}{l} \\
\text { Oct. } 22 \\
\text { Jan. } \quad 6\end{array}$ & $\begin{array}{l}24.61 \\
18.66 \\
\end{array}$ & $\begin{array}{r}14,900 \\
4,000 \\
\end{array}$ & $\begin{array}{l}1951 \\
1952 \\
1953 \\
\end{array}$ & $\begin{array}{ll}\text { Nov. } & 19 \\
\text { Feb. } & 20 \\
\text { Aug. } & 29 \\
\end{array}$ & $\begin{array}{l}15.14 \\
15.90 \\
20.44 \\
\end{array}$ & $\begin{array}{l}1,110 \\
1,450 \\
6,120\end{array}$ \\
\hline
\end{tabular}

(115) Santa Fe River near High Springs, Fla.

Location.--Lat $29^{\circ} 51^{\prime}$, long $82^{\circ} 38^{\prime}$, in sec. 29, T, 7 S., R. 17 E., near right bank at upstream s1de of bridge on U. S. Highway $27,150 \mathrm{ft}$ upstream from Atlant1c Coast L1ne Ra1lroad bridge, and 2 miles northwest of High Springs.

Drainage area. $--950 \mathrm{sq} \mathrm{ml}$, approximately.

Records avallable.--U. S. Geologlcal Survey: January 1931 to December 1953, da1ly gage helghts and discharges.

Gage.--Water-stage recorder. Datum of gage is $26.36 \mathrm{ft}$ above mean sea level, datum of 1929 (ievels by Florida State Road Department). Prior to Jan. 9, 1933, staff gage at same site and datum.

Stage-discharge relation.--Defined by current-meter measurements, considerable shifting at medium and low stages. Extremely high stages of the Suwannee River causes backwater at the station.

Annual peak stages and discharges

\begin{tabular}{|c|c|c|c|c|c|c|c|}
\hline $\begin{array}{c}\text { Calendar } \\
\text { year }\end{array}$ & Date & $\begin{array}{l}\text { Gage } \\
\text { height } \\
\text { (feet) }\end{array}$ & $\begin{array}{l}\text { Discharge } \\
\text { (cfs) }\end{array}$ & $\begin{array}{c}\text { Calendar } \\
\text { year }\end{array}$ & Date & $\begin{array}{c}\text { Gage } \\
\text { height } \\
\text { (feet) }\end{array}$ & $\begin{array}{l}\text { Discharge } \\
\text { (cfs) }\end{array}$ \\
\hline $\begin{array}{l}1931 \\
1932 \\
1933 \\
1934 \\
1935\end{array}$ & $\begin{array}{ll}\text { Apr. } & 10 \\
\text { Sept. } & 19 \\
\text { Sept. } 11 \\
\text { June } 18 \\
\text { Sept. } 10\end{array}$ & $\begin{array}{r}3.84 \\
3.44 \\
6.10 \\
14.90 \\
11.10\end{array}$ & $\begin{array}{r}1,480 \\
1,230 \\
2,760 \\
11,800 \\
6,830\end{array}$ & $\begin{array}{l}1943 \\
1944 \\
1945 \\
1946\end{array}$ & $\begin{array}{ll}\text { Aug. } & 22,23 \\
\text { Oct. } 23 & \\
\text { Aug. } & 25\end{array}$ & $\begin{array}{r}3.57 \\
13.23 \\
6.36\end{array}$ & $\begin{array}{l}1,180 \\
9,560 \\
2,940\end{array}$ \\
\hline 1935 & Sept. 10 & 11.10 & 6,830 & $\begin{array}{l}1946 \\
1947\end{array}$ & $\begin{array}{l}\text { Aug. } 4,5 \\
\text { Sept. } 28^{5}\end{array}$ & $\begin{array}{r}6.73 \\
12.62\end{array}$ & $\begin{array}{l}3,140 \\
8,800\end{array}$ \\
\hline $\begin{array}{l}1936 \\
1937 \\
1938 \\
1939\end{array}$ & $\begin{array}{lr}\text { Apr. } & 8 \\
\text { Oct. } & 7 \\
\text { Oct. } & 29 \\
\text { Aug. } & 12\end{array}$ & $\begin{array}{l}3.84 \\
8.85 \\
8.41 \\
4.65\end{array}$ & $\begin{array}{l}1,490 \\
4,610 \\
4,280 \\
1,890\end{array}$ & $\begin{array}{l}1948 \\
1949 \\
1950\end{array}$ & $\begin{array}{lr}\text { Mar. } & 14 \\
\text { Sept. } & 5 \\
\text { Sept. } & 11\end{array}$ & $\begin{array}{r}15.71 \\
9.34 \\
11.64\end{array}$ & $\begin{array}{r}12,700 \\
5,080 \\
7,600\end{array}$ \\
\hline 1940 & Aug. $\quad 28$ & 3.99 & 1,470 & $\begin{array}{l}1951 \\
1952\end{array}$ & $\begin{array}{l}\text { Nov. } 21 \\
\text { Mar. } 1\end{array}$ & $\begin{array}{l}3.21 \\
3.77\end{array}$ & $\begin{array}{l}1,060 \\
1,220\end{array}$ \\
\hline $\begin{array}{l}1941 \\
1942 \\
\end{array}$ & $\begin{array}{ll}\text { Oct. } & 25 \\
\text { Mar. } & 11\end{array}$ & $\begin{array}{r}11.90 \\
7.93 \\
\end{array}$ & $\begin{array}{l}7,960 \\
3,930 \\
\end{array}$ & 1953 & Dec. & 8.10 & 4,040 \\
\hline
\end{tabular}


Suwannee River Basin

(116) Santa Fe River near Fort White, Fla.

Location.--tat $29^{\circ} 51^{\prime}$, long $82^{\circ} 43^{\prime}$, in sec. 28 , T. 7 S., R. 16 E., on left bank 2 miles upstream from bridge on State Highway 47, 5 miles south of Fort White, and 15 miles upstream from mouth.

Drainage area.--1,080 sq $\mathrm{mi}$, approximately.

Records avallable.--Mees and Mees, Inc.: July 1925 to September 1927, daily gage helghts and discharges. U. S. Geological Survey: October 1927 to January 1930 and June 1932 to December 1953, dally gage heights and discharges.

Gage.--Water-stage recorder. Datum of gage is $20.8 \dot{6} \mathrm{ft}$ above mean sea level (levels by Corps of Engineers). Prior to June 4, 1932, staff gage at several sites within 200 ft of present site and at various datums.

Oct. 1, 1947, to Feb. 10, 1949, auxillary wire-weight gage and since Feb. 11, 1949, waterstage recorder at bridge on State Highway 49, 13.1 miles downstream. Datum of auxiliary gage is $3.5 \mathrm{ft}$ above mean sea level, datum of 1929 .

Stage-discharge relation.--Defined by current-meter measurements; affected by backwater from Suwannee River. D1scharge is computed using fall as a factor.

Annual peak stages and discharges

\begin{tabular}{|c|c|c|c|c|c|c|c|}
\hline $\begin{array}{c}\text { Calendar } \\
\text { year }\end{array}$ & Date & $\begin{array}{c}\text { Gage } \\
\text { height } \\
\text { (feet) }\end{array}$ & $\begin{array}{c}\text { Discharge } \\
\text { (cfs) }\end{array}$ & $\begin{array}{l}\text { Calendar } \\
\text { year }\end{array}$ & Date & $\begin{array}{l}\text { Gage } \\
\text { height } \\
\text { (feet) }\end{array}$ & $\begin{array}{c}\text { Discharge } \\
\text { (cfs) }\end{array}$ \\
\hline $\begin{array}{l}1926 \\
1927 \\
1928 \\
1929 \\
\end{array}$ & $\begin{array}{lc}\text { Aug. } & 3-6 \\
\text { Mar. } & 1-7 \\
\text { Aug. } & 27-29 \\
\text { Oct. } & 3-6 \\
\end{array}$ & $\begin{array}{l}4.8 \\
3.0 \\
9.5 \\
6.9 \\
\end{array}$ & $\begin{array}{l}4,120 \\
2,370 \\
4,750 \\
4,180 \\
\end{array}$ & $\begin{array}{l}1942 \\
1943 \\
1944\end{array}$ & $\begin{array}{ll}\text { Mar: } & 11-13 \\
\text { Mar: } & 23 \\
\text { Aug. } & 23 \\
\text { Oct. } & 24\end{array}$ & $\begin{array}{l}- \\
6.58 \\
1.95 \\
9.48\end{array}$ & $\begin{array}{l}4,750 \\
1,760 \\
9,300\end{array}$ \\
\hline $\begin{array}{l}1932 \\
1933 \\
1934 \\
1935\end{array}$ & $\begin{array}{ll}\text { Sept. } 20 \\
\text { Apr. } 20 \\
\text { June } 20 \\
\text { Sept. } 10\end{array}$ & $\begin{array}{r}- \\
4.19 \\
11.04 \\
8.26\end{array}$ & $\begin{array}{r}1,740 \\
2,810 \\
11,400 \\
6,680\end{array}$ & $\begin{array}{l}1940 \\
1946 \\
1947 \\
1948\end{array}$ & $\begin{array}{lr}\text { Aug. } & \text { C6 } \\
\text { Aug. } & 6 \\
\text { Sept. } & 29 \\
\text { Mar. } & 14 \\
\text { Apr. } & 12\end{array}$ & $\begin{array}{c}4.82 \\
4.79 \\
8.73 \\
-7 \\
13.70\end{array}$ & $\begin{array}{r}3,650 \\
3,820 \\
8,110 \\
12,300\end{array}$ \\
\hline $\begin{array}{l}1936 \\
1937 \\
1938 \\
1939 \\
1940 \\
1941\end{array}$ & $\begin{array}{ll}\text { Feb. } & 13,14 \\
\text { Oct. } & 7,8 \\
\text { Oct. } & 30 \\
\text { Aug. } & 12 \\
\text { Aug. } & 28,29 \\
\text { Oct. } & 26\end{array}$ & $\begin{array}{l}2.46 \\
6.00 \\
5.51 \\
2.84 \\
2.16 \\
8.14\end{array}$ & $\begin{array}{l}2,140 \\
4,730 \\
4,250 \\
2,480 \\
2,000 \\
7,410\end{array}$ & $\begin{array}{l}1949 \\
1950 \\
1951 \\
1952 \\
1953\end{array}$ & $\begin{array}{lr}\text { Sept. } & 6 \\
\text { Oct. } & 26 \\
& \\
\text { Nov. } & 23 \\
\text { Mar. } & 4 \\
\text { Dec. } & 31\end{array}$ & $\begin{array}{l}6.76 \\
8.16 \\
1.64 \\
2.29 \\
5.85\end{array}$ & $\begin{array}{l}5,510 \\
7,520 \\
1,670 \\
2,020 \\
5,080\end{array}$ \\
\hline
\end{tabular}

(117) Suwannee River near Bell, Fla.

Location.--Lat $29^{\circ} 48^{\prime}$, long $82^{\circ} 55^{\prime}$, in sec. 16 or 17, T. 8 S., R. 14 E., on left bank at Rock Bluff Ferry, $4 \frac{1}{2}$ miles northwest of Bell and 10 miles downstream from Santa Fe River.

Dra1nage area.--9,260 sq $\mathrm{ml}$, approximately.

Records avallable.--U. S. Geological Survey: June 1932 to December 1953, daily gage helghts and discharges.

Gage.-Water-stage recorder. Datum of gage is $3.60 \mathrm{ft}$ above mean sea level (levels by Corps of Engineers).

Stage-discharge relation.--Defined by current-meter measurements.

迎storical data.-Flood of Aug. 28, 1928, reached stage of about $25.9 \mathrm{ft}$, from information by a local resldent. 
Suwannee R1ver Basin

(117) Suwannee River near Bell, Fla.--Cont1nued

Annual peak stages and discharges

\begin{tabular}{|c|c|c|c|c|c|c|c|}
\hline $\begin{array}{c}\text { Calendar } \\
\text { year }\end{array}$ & Date & $\begin{array}{c}\text { Gage } \\
\text { height } \\
\text { (feet) }\end{array}$ & $\begin{array}{c}\text { Discharge } \\
\text { (cfs) }\end{array}$ & $\begin{array}{c}\text { Calendar } \\
\text { year }\end{array}$ & Date & $\begin{array}{l}\text { Gage } \\
\text { height } \\
\text { (feet) }\end{array}$ & $\begin{array}{c}\text { Discharge } \\
\text { (cfs) }\end{array}$ \\
\hline 1928 & Aug. 28 & 25.9 & - & 1942 & Mar. 28 & 17.28 & 30,000 \\
\hline $\begin{array}{l}1932 \\
1933 \\
1934\end{array}$ & $\begin{array}{lr}\text { Sept. } & 30 \\
\text { Mar. } & 2 \\
\text { June } & 21\end{array}$ & $\begin{array}{l}12.21 \\
15.36 \\
11.30\end{array}$ & $\begin{array}{l}16,100 \\
24,500 \\
14,800\end{array}$ & $\begin{array}{l}1944 \\
1945\end{array}$ & $\begin{array}{l}\text { Apr. } \\
\text { Sept. } 3\end{array}$ & $\begin{array}{l}15.26 \\
15.98\end{array}$ & $\begin{array}{r}8,270 \\
24,000 \\
25,300\end{array}$ \\
\hline 1935 & Sept. 25 & 14.63 & 22,100 & $\begin{array}{l}1946 \\
1947\end{array}$ & $\begin{array}{ll}\text { Aug. } & 19 \\
& \end{array}$ & $\begin{array}{l}13.59 \\
19.52\end{array}$ & 18,800 \\
\hline $\begin{array}{l}1936 \\
1937 \\
1938 \\
1939\end{array}$ & $\begin{array}{lr}\text { Mar. } & 2 \\
\text { Apr. } & 22 \\
\text { Aug. } & 15 \\
\text { Mar. } & 16\end{array}$ & $\begin{array}{r}12.09 \\
15.53 \\
6.40 \\
10.28\end{array}$ & $\begin{array}{r}16,200 \\
24,800 \\
8,130 \\
13,600\end{array}$ & $\begin{array}{l}1948 \\
1949 \\
1950\end{array}$ & $\begin{array}{l}\text { Apr. } 13 \\
\text { Sept. } 12 \\
\text { Oct. } 28\end{array}$ & $\begin{array}{r}27.43 \\
14.00 \\
9.56\end{array}$ & $\begin{array}{l}82,300 \\
18,800 \\
11,700\end{array}$ \\
\hline 1940 & Feb. 28-Mar. 1 & 8.88 & 11,700 & $\begin{array}{l}1951 \\
1952\end{array}$ & $\begin{array}{ll}\text { Apr. } & 12 \\
\text { Mar. } & 11\end{array}$ & $\begin{array}{r}8.85 \\
12.25\end{array}$ & 10,700 \\
\hline 1941 & Aug. & 7.14 & 9,110 & 1953 & oct. 12 & 14.33 & 20,700 \\
\hline
\end{tabular}

(118) Suwannee R1ver near W1lcox, Fla.

Location.--Lat $29^{\circ} 36^{\prime}$, long $82^{\circ} 56^{\prime}$, in sec. 29, T. $10 \mathrm{~S}$, , R. $14 \mathrm{E}$., on left bank about $400 \mathrm{ft}$ downstream from Fort Fannin bridge on U. S. Highway 19, and 2 miles southwest of W1lcox.

Drainage area.--About $9,500 \mathrm{sq} \mathrm{ml}$.

Records avallable.--U. S. Geological Survey: September 1930 to September 1931 and March 1942 to December 1953, gage heights and discharges (fragimentary March 1942 to January 1951).

Gage.--Water-stage recorder. Datum of gage 1s at mean sea level, datum of 1929. Prior to July 4 1931, staff gage at s1te $400 \mathrm{ft}$ upstream at same datum. July 4 to Sept. 30,1931 , and Mar. 26 to May 14, 1942, water-stage recorder at present s1te and datum. May 15, 1942, to Jan. 24, 1951, staff gage at present s1te and datum. Staff gage read once da1ly when stage exceeded $6.0 \mathrm{ft}$. S1nce Feb. 1, 1951, aux1l1ary water-stage recorder about 9 m1les downstream.

Stage-discharge relation.--Defined by current-meter measurements. Flow affected by tide for discharges less than $12,000 \mathrm{cfs}$. Discharges computed using fall as a factor below $12,000 \mathrm{cfs}$.

Historlcal data.--Flood of August 1928 reached stage of about $20.0 \mathrm{ft}$ (source of information unknown).

Annual peak stages and discharges

\begin{tabular}{|c|c|c|c|c|c|c|c|}
\hline $\begin{array}{c}\text { Calendar } \\
\text { year }\end{array}$ & Date & $\begin{array}{l}\text { Gage } \\
\text { height } \\
\text { (feet) }\end{array}$ & $\begin{array}{c}\text { Discharge } \\
\text { (cfs) }\end{array}$ & $\begin{array}{c}\text { Calendar } \\
\text { year }\end{array}$ & Date & $\begin{array}{l}\text { Gage } \\
\text { height } \\
\text { (feet) }\end{array}$ & $\begin{array}{c}\text { Discharge } \\
\text { (cfs) }\end{array}$ \\
\hline 1928 & $\begin{array}{l}\text { Aug. }- \\
\text { Feb. } 1\end{array}$ & $\begin{array}{l}20.0 \\
6.60\end{array}$ & 14,500 & $\begin{array}{l}1947 \\
1948 \\
1949 \\
1950\end{array}$ & $\begin{array}{ll}\text { Nov. } & 26-29 \\
\text { Apr: } & 14 \\
\text { Sept. } & 13-15 \\
\text { Oct. } & 29\end{array}$ & $\begin{array}{r}15.15 \\
22.32 \\
9.90 \\
6.40\end{array}$ & $\begin{array}{l}37,800 \\
84,700 \\
20,100 \\
12,400\end{array}$ \\
\hline $\begin{array}{l}1942 \\
1944 \\
1945\end{array}$ & $\begin{array}{l}\text { Mar. } 28-31 \\
\text { Apr. } 13 \\
\text { Sept. } 3,4\end{array}$ & $\begin{array}{l}12.96 \\
10.68 \\
11.40\end{array}$ & $\begin{array}{l}29,200 \\
22,300 \\
24,300\end{array}$ & $\begin{array}{l}1951 \\
1952 \\
1953\end{array}$ & $\begin{array}{ll}\text { Apr. } & 14 \\
\text { Mar. } & 11 \\
\text { Oct. } & 13,14\end{array}$ & $\begin{array}{l}- \\
8.43 \\
9.87\end{array}$ & $\begin{array}{l}12,000 \\
16,700 \\
20,000\end{array}$ \\
\hline 1946 & Aug. 19 & 9.27 & 18,600 & & & & 1 \\
\hline
\end{tabular}




\section{Fenhol loway River Basin}

(119) Fenholloway R1ver at Foley, Fla.

Location.--Lat $30^{\circ} 04^{\prime}$, long $83^{\circ} 32^{\prime}$, in sec. 9, T. 5 S., R. 8 E., on right bank at upstream side of highway bridge in Foley.

Dra1nage area.--180 sq $\mathrm{m} 1$, approximately.

Records avallable.--U. S. Geological Survey: September 1946 to December 1953, da1ly gage helghts and discharges.

Gage.--Water-stage recorder. Datum of gage is $29.36 \mathrm{ft}$ above mean sea level, datum of 1929. Prior to June 24,1947 , staff gage at same site and datum.

Stage-discharge relation.--Defined by current-meter measurements. Considerable shifting at medium and low stages.

Annual peak stages and discharges

\begin{tabular}{|c|c|c|c|c|c|c|c|}
\hline $\begin{array}{c}\text { Calendar } \\
\text { year }\end{array}$ & Date & $\begin{array}{l}\text { Gage } \\
\text { height } \\
\text { (feet) }\end{array}$ & $\begin{array}{c}\text { Discharge } \\
\text { (cfs) }\end{array}$ & $\begin{array}{c}\text { Calendar } \\
\text { year }\end{array}$ & Date & $\begin{array}{l}\text { Gage } \\
\text { height } \\
\text { (feet) }\end{array}$ & $\begin{array}{c}\text { Discharge } \\
\text { (cfs) }\end{array}$ \\
\hline $\begin{array}{l}1947 \\
1948 \\
1949 \\
1950\end{array}$ & $\begin{array}{lr}\text { Nov. } 12 \\
\text { Mar. } 10 \\
\text { Feb. } 8 \\
\text { July } 10\end{array}$ & $\begin{array}{l}14.46 \\
16.03 \\
13.80 \\
11.37\end{array}$ & $\begin{array}{r}1,140 \\
2,640 \\
826 \\
216\end{array}$ & $\begin{array}{l}1951 \\
1952 \\
1953\end{array}$ & $\begin{array}{ll}\text { Nov. } & 18 \\
\text { Feb. } & 18 \\
\text { Sept. } & 28\end{array}$ & $\begin{array}{l}13.92 \\
13.66 \\
15.32\end{array}$ & $\begin{array}{r}842 \\
725 \\
1,860\end{array}$ \\
\hline
\end{tabular}

Ochlockonee River Basin

(120) Ochlockonee River near Thomasville, Ga.

Location.--Lat $30^{\circ} 52^{\prime}$, long $84^{\circ} 03^{\prime}$, on downstream side of left bank pier of bridge on U. S. H1ghway 84,2 miles upstream from Atlantic Coast Line Railroad bridge, 4 miles upstream from Barnetts Creek, 5 miles northwest of Thomasville, Thomas County, and 6 miles downstream from Little Ochlockonee Rlver.

Drainage area.--550 sq $\mathrm{ml}$, approximately.

Records ava1lable.--U. S. Geological Survey: August 1937 to December 1953, da1ly gage heights and discharges.

Gage.-Water-stage recorder. Datum of gage is $133.6 \mathrm{ft}$ above mean sea level, datum of 1929 , supplementary adjustment of 1936. Prior to Jan. 7, 1947, wire-weight gage at same site and datum.

Stage-discharge relation.--Defined by current-meter measurements below 25,000 cfs and extended above by logarithmic plotting.

Historical data.--Fiood of Apr1l 1948 was reported to be the highest in the memory of residents of the area. 
Ochlockonee River Basin

(120) Ochlockonee River near Thomasville, Ga.--Continued

Annual peak stages and discharges

\begin{tabular}{|c|c|c|c|c|c|c|c|}
\hline $\begin{array}{c}\text { Calendar } \\
\text { year }\end{array}$ & Date & $\begin{array}{c}\text { Gage } \\
\text { height } \\
\text { (feet) }\end{array}$ & $\begin{array}{c}\text { Discharge } \\
\text { (cfs) }\end{array}$ & $\begin{array}{c}\text { Calendar } \\
\text { year }\end{array}$ & Date & $\begin{array}{l}\text { Gage } \\
\text { height } \\
\text { (feet) }\end{array}$ & $\begin{array}{c}\text { Discharge } \\
\text { (cfs) }\end{array}$ \\
\hline $\begin{array}{l}1937 \\
1938 \\
1939 \\
1940 \\
1941 \\
1942 \\
1943 \\
1944 \\
1945 \\
\end{array}$ & $\begin{array}{lr}\text { Sept. } & 21 \\
\text { Mar. } & 22 \\
\text { Mar. } & 3 \\
\text { Feb. } & 21 \\
& \\
\text { Mar. } & 10 \\
\text { Jan. } & 5 \\
\text { Mar. } & 9 \\
\text { Mar. } & 8 \\
\text { July } & 12 \\
\end{array}$ & $\begin{array}{r}17.6 \\
8.8 \\
14.0 \\
10.7 \\
10.4 \\
17.8 \\
14.8 \\
17.8 \\
15.3 \\
\end{array}$ & $\begin{array}{l}9,090 \\
1,310 \\
3,240 \\
1,820 \\
1,730 \\
9,900 \\
4,040 \\
9,900 \\
4,680 \\
\end{array}$ & $\begin{array}{l}1946 \\
1947 \\
1948 \\
1949 \\
1950 \\
1951 \\
1952 \\
1953\end{array}$ & $\begin{array}{lr}\text { May } & 22 \\
\text { Apr. } & 17 \\
\text { Apr. } & 2 \\
\text { Apr. } & 15 \\
\text { Mar. } 17 \\
\\
\text { Apr. } \\
\text { Mar. } 27 \\
\text { Apr. } 14\end{array}$ & $\begin{array}{l}15.8 \\
17.0 \\
29.1 \\
\overline{11.9} \\
13.2 \\
13.8 \\
15.6\end{array}$ & $\begin{array}{r}5,480 \\
7,920 \\
72,000 \\
3,900 \\
2,090 \\
2,660 \\
3,000 \\
5,140\end{array}$ \\
\hline
\end{tabular}

(121) T1red Creek near Ca1ro, Ga.

Location.--Lat $30^{\circ} 54^{\prime}$, long $84^{\circ} 16^{\prime}$, on left bank $140 \mathrm{ft}$ upstream from h1ghway bridge, a quarter of a mile downstream from Wolf Creek, l mile downstream from Atlant1c Coast Line Ra1lroad bridge, and 3 miles west of Calro, Grady County.

Dra1nage area. $--55 \mathrm{sq} \mathrm{m}$, approximately.

Records ava1lable.--U. S. Geologlcal Survey: July 1943 to December 1953, da1ly gage he1ghts and discharges.

Gage.--Water-stage recorder. Datum of gage is $159.0 \mathrm{ft}$ above mean sea level, datum of 1929 , supplementary adjustment of 1936.

Stage-discharge relation.--Defined by current-meter measurements below 3,000 cfs and by slopearea measurement at 28,000 cfs.

H1storical data.--Flood of Apr11 1948 was reported to be the highest in memory of residents of the area.

Annual peak stages and discharges

\begin{tabular}{c|c|c|c||c|c|c|c}
\hline $\begin{array}{c}\text { Calendar } \\
\text { year }\end{array}$ & Date & $\begin{array}{c}\text { Gage } \\
\text { height } \\
\text { (feet) }\end{array}$ & $\begin{array}{c}\text { Discharge } \\
\text { (cfs) }\end{array}$ & $\begin{array}{c}\text { Calendar } \\
\text { year }\end{array}$ & Date & $\begin{array}{c}\text { Gage } \\
\text { height } \\
\text { (feet) }\end{array}$ & $\begin{array}{c}\text { Discharge } \\
\text { (cfs) }\end{array}$ \\
\hline 1944 & Mar. 7 & 9.2 & 4,640 & 1949 & Apr. 12 & 7.38 & 1,850 \\
1945 & Apr. 23 & 8.3 & 3,100 & 1950 & July 7 & 7.30 & 1,730 \\
1946 & Mar. 17 & 10.5 & 7,300 & 1951 & Mar. 19 & 7.27 & 1,670 \\
1947 & Apr. 16 & 8.2 & 2,940 & 1952 & Sept. 22 & 6.55 & 955 \\
1948 & Apr. 1 & 16.3 & 28,100 & 1953 & Apr. 7 & 7.53 & 1,940 \\
\hline
\end{tabular}




\section{Ochlockonee R1ver Bas1n}

(122) Ochlockonee River near Havana, Fla.

Location.--Lat $30^{\circ} 33^{\prime}$, long $84^{\circ} 23^{\prime}$, in sec. 24 , T. 2 N., R. 2 W., on upstream side near center of span of bridge on U. S. Highway 90, three-quarters of a mile upstream from Seaboard Air Line Rallroad bridge, 4 miles downstream from Mill Creek, and 5 miles southeast of Havana.

Dralnage area. $--1,020 \mathrm{sq} \mathrm{ml}$, approximately.

Records avallable.--West Florida Power Co.: September 1921 to December 1928, daily gage helghts. U. S. Geolog1cal Survey: December 1928 to December 1953, da1ly gage helghts and discharges.

Gage.--Wire-welght gage read once dally. Datum of gage 1s $59.16 \mathrm{ft}$ above mean sea level, datum of 1929. Dec. 13, 1928, to Aug. 11, 1934, cha1n gage at same site and datum. Prior to Dec. 13, 1928, staff gage at datum $60.00 \mathrm{ft}$ lower.

Stage-discharge relation.--Defined by current-meter measurements. Annual peak discharges prior to 1929 computed from rating developed during period 1926 to 1953.

Historical data.--Flood of April 1948 was reported to be highest in the memory of residents of the area.

Annual peak stages and discharges

\begin{tabular}{|c|c|c|c|c|c|c|c|}
\hline $\begin{array}{c}\text { Calendar } \\
\text { year }\end{array}$ & Date & $\begin{array}{l}\text { Gage } \\
\text { height } \\
\text { (feet) }\end{array}$ & $\begin{array}{c}\text { Discharge } \\
\text { (cfs) }\end{array}$ & $\begin{array}{c}\text { Calendar } \\
\text { year }\end{array}$ & Date & $\begin{array}{l}\text { Gage } \\
\text { height } \\
\text { (feet) }\end{array}$ & $\begin{array}{c}\text { Discharge } \\
\text { (cfs) }\end{array}$ \\
\hline $\begin{array}{l}1922 \\
1923 \\
1924 \\
1925\end{array}$ & $\begin{array}{lr}\text { June } & 4 \\
\text { Aug. } & 30 \\
\text { Oct. } & 3 \\
\text { Jan. } & 23\end{array}$ & $\begin{array}{r}28.1 \\
27.9 \\
27.6 \\
a 28.1\end{array}$ & $\begin{array}{l}9,400 \\
8,910 \\
8,210 \\
9,400\end{array}$ & $\begin{array}{l}1938 \\
1939 \\
1940\end{array}$ & $\begin{array}{lr}\text { Mar: } & 25 \\
\text { Mar: } & 8 \\
\text { Feb: } & 23\end{array}$ & $\begin{array}{l}19.76 \\
22.76 \\
22.35\end{array}$ & $\begin{array}{l}1,760 \\
3,150 \\
2,950\end{array}$ \\
\hline $\begin{array}{l}1926 \\
1927 \\
1928 \\
1929 \\
1930\end{array}$ & $\begin{array}{ll}\text { Sept. } 25 & \\
\text { Feb. } 25 \\
\text { Aug. } 17 \text { or } 18 \\
\text { Mar. } 19 \\
\text { Apr. } 10\end{array}$ & $\begin{array}{l}27.4 \\
19.3 \\
31.7 \\
30.3 \\
27.07\end{array}$ & $\begin{array}{r}7,770 \\
1,520 \\
21,300 \\
15,300 \\
7,160\end{array}$ & $\begin{array}{l}1941 \\
1942 \\
1943 \\
1944 \\
1945\end{array}$ & $\begin{array}{lr}\text { Mar. } & 13 \\
\text { Jan. } & 7 \\
\text { Mar. } & 9 \\
\text { Mar. } & 12 \\
\text { May } & 1\end{array}$ & $\begin{array}{l}21.59 \\
29.58 \\
27.28 \\
28.38 \\
26.74\end{array}$ & $\begin{array}{r}2,610 \\
14,100 \\
7,570 \\
10,300 \\
6,460\end{array}$ \\
\hline $\begin{array}{l}1931 \\
1932 \\
1933\end{array}$ & $\begin{array}{lr}\text { May } & 9 \\
\text { Sept. } & 21 \\
\text { Nov. } & 5 \\
\text { Apr. } & 17\end{array}$ & $\begin{array}{c}23.10 \\
- \\
26.31 \\
27.83\end{array}$ & $\begin{array}{c}3,190 \\
5,680 \\
8,670\end{array}$ & $\begin{array}{l}1946 \\
1947 \\
1948 \\
1949 \\
1950\end{array}$ & $\begin{array}{lr}\text { Mar. } & 21 \\
\text { Dec. } & 18 \\
\text { Apr. } & 4 \\
\text { Apr. } & 17 \\
\text { Sept. } & 1\end{array}$ & $\begin{array}{l}27.05 \\
28.16 \\
35.08 \\
26.07 \\
22.64\end{array}$ & $\begin{array}{r}6,990 \\
9,420 \\
55,900 \\
5,130 \\
2,560\end{array}$ \\
\hline $\begin{array}{l}1934 \\
1935\end{array}$ & $\begin{array}{ll}\text { June } & 10 \\
\text { Sept. } & 10\end{array}$ & $\begin{array}{l}17.44 \\
27.96\end{array}$ & $\begin{array}{l}1,230 \\
9,150\end{array}$ & $\begin{array}{l}1951 \\
1952\end{array}$ & $\begin{array}{lr}\text { Apr. } & 5 \\
\text { Feb. } & 27\end{array}$ & $\begin{array}{l}23.64 \\
25.36\end{array}$ & $\begin{array}{l}3,040 \\
4,620\end{array}$ \\
\hline $\begin{array}{l}1936 \\
1937\end{array}$ & $\begin{array}{ll}\text { Apr. } & 16 \\
\text { Sept. } 24\end{array}$ & $\begin{array}{l}26.20 \\
28.42\end{array}$ & $\begin{array}{r}6,280 \\
10,100 \\
\end{array}$ & 1953 & Apr. $\quad 18$ & 26.35 & 5,920 \\
\hline
\end{tabular}

a May have been higher during period of no gage-helght record.

(123) Ochlockonee River near Bloxham, Fla.


left pler of bridge on State Highway 20,3,000 ft downstream from powerplant and dam, and $1 \frac{1}{2}$ miles southwest of Bloxham.

Dra1nage area. - - 1,660 sq $\mathrm{m1}$, approximately.

Records available.--U. S. Geological Survey: June 1926 to December 1953, daily gage helghts and discharge.

Gage.--Water-stage recorder. Datum of gage is $29.69 \mathrm{ft}$ above mean sea level, datum of 1929. Prior to Apr.9, 1930, staff gage at site 2,700 ft upstream at same datum. Apr. 9, 1930, to Jan. 19, 1939, water-stage recorder at site 2,000 $\mathrm{ft}$ upstream at same datum.

Stage-discharge relation.--Defined by current-meter measurements below 38,800 cfs. Since 1929 flow regulated by powerplant above station and storage in Lake Talquin. 
Ochlockonee R1ver Bas1n

(123) Ochlockonee River near Bloxham, Fla.--Cont1nued

Annual peak stages and discharges

\begin{tabular}{|c|c|c|c|c|c|c|c|}
\hline $\begin{array}{c}\text { Calendar } \\
\text { year }\end{array}$ & Date & $\begin{array}{l}\text { Gage } \\
\text { height } \\
\text { (feet) }\end{array}$ & $\begin{array}{c}\text { Discharge } \\
\text { (cfs) }\end{array}$ & $\begin{array}{l}\text { Calendar } \\
\text { year }\end{array}$ & Date & $\begin{array}{l}\text { Gage } \\
\text { height } \\
\text { (feet) }\end{array}$ & $\begin{array}{l}\text { Discharge } \\
\text { (cfs) }\end{array}$ \\
\hline $\begin{array}{l}1926 \\
1927 \\
1928 \\
1929 \\
1930\end{array}$ & $\begin{array}{ll}\text { Sept. } & 27 \\
\text { Feb. } & 27 \\
\text { Aug. } & 19 \\
\text { Mar. } & 22 \\
\text { Apr. } & 12\end{array}$ & $\begin{array}{l}15.5 \\
7.15 \\
21.4 \\
17.61 \\
-\end{array}$ & $\begin{array}{r}8,050 \\
2,380 \\
19,900 \\
10,400 \\
a_{7}, 840\end{array}$ & $\begin{array}{l}1941 \\
1942 \\
1943 \\
1944 \\
1945\end{array}$ & $\begin{array}{l}\text { Mar. } 11 \\
\text { Jan. } 8 \\
\text { Mar. } 7 \\
\text { Mar. } 13,30 \\
\text { May } 4\end{array}$ & $\begin{array}{l}- \\
- \\
-\end{array}$ & $\begin{array}{r}a 3,120 \\
a 13,300 \\
a 12,600 \\
a 10,200 \\
a 6,600\end{array}$ \\
\hline $\begin{array}{l}1931 \\
1932 \\
1933 \\
1934 \\
1935\end{array}$ & $\begin{array}{lr}\text { Jan. } & 23 \\
\text { Nov. } & 6 \\
\text { Apr. } & 15 \\
\text { July } & 21 \\
\text { Sept. } & 13\end{array}$ & - & $\begin{array}{r}a_{2}, 800 \\
a_{8}, 050 \\
a_{10,500} \\
a 1,830 \\
a 9,020\end{array}$ & $\begin{array}{l}1946 \\
1947 \\
1948 \\
1949 \\
1950\end{array}$ & $\begin{array}{lr}\text { Mar. } & 18 \\
\text { Apr. } 17 \\
\text { Apr. } 5 \\
\text { Apr. } 13 \\
\text { Apr. } 5\end{array}$ & $\begin{array}{c}- \\
23.50 \\
- \\
-\end{array}$ & $\begin{array}{r}a_{12}, 500 \\
a_{12}, 500 \\
50,200 \\
a 9,450 \\
a_{4,260}\end{array}$ \\
\hline $\begin{array}{l}1936 \\
1937 \\
1938 \\
1939 \\
1940 \\
\end{array}$ & $\begin{array}{lr}\text { Feb. } & 14 \\
\text { Sept. } & 2 \\
\text { Mar. } & 9 \\
\text { June } & 15 \\
\text { Feb. } & 23-26 \\
\end{array}$ & $\begin{array}{l}- \\
- \\
-\end{array}$ & $\begin{array}{r}a 6,390 \\
a 16,100 \\
a 2,510 \\
a 7,980 \\
a 3,470 \\
\end{array}$ & $\begin{array}{l}1951 \\
1952 \\
1953\end{array}$ & $\begin{array}{lr}\text { Apr. } & 2 \\
\text { Feb. } 24 \\
\text { Apr. } 19\end{array}$ & $\begin{array}{l}- \\
-\end{array}$ & $\begin{array}{l}a 3,850 \\
a 6,250 \\
a 5,670\end{array}$ \\
\hline
\end{tabular}

Apalachicola River Basin

(124) Spring Creek near Iron C1ty, Ga.

Location.--Lat $31^{\circ} 03^{\prime}$, long $84^{\circ} 43^{\prime}$, on right bank $125 \mathrm{ft}$ downstream from highway bridge, $1 \frac{1}{2} \mathrm{mlles}$ downstream from Aycock Creek, $1 \frac{1}{2}$ m1les upstream from Dry Creek, 5 miles north of Brinson, and $5 \frac{1}{2}$ miles northeast of Iron City, Seminole County.

Drainage area.--520 sq $\mathrm{ml}$, approximately.

Records avallable.--U. S. Geological Survey: October 1920 to June 1921, June 1937 to December 1953 , dally gage helghts and discharges.

Gage.--Water-stage recorder. Datum of gage is $85.7 \mathrm{ft}$ above mean sea level, datum of 1929 , supplementary adjustment of 1936. Prior to Oct. 18, 1952, staff gage at s1te 125 ft upstream at same datum. Oct. 21 , 1920, to June 30,1921 , at different datum.

Stage-d1scharge relation.--Defined by current-meter measurements below 8,000 cfs and extended above by logarithmic plotting.

Annual peak stages and discharges

\begin{tabular}{|c|c|c|c|c|c|c|c|}
\hline $\begin{array}{c}\text { Calendar } \\
\text { year }\end{array}$ & Date & $\begin{array}{l}\text { Gage } \\
\text { height } \\
\text { (feet) }\end{array}$ & $\begin{array}{l}\text { Discharge } \\
\text { (cfs) }\end{array}$ & $\begin{array}{c}\text { Calendar } \\
\text { year }\end{array}$ & Date & $\begin{array}{l}\text { Gage } \\
\text { height } \\
\text { (feet) }\end{array}$ & $\begin{array}{c}\text { Discharge } \\
\text { (cfs) }\end{array}$ \\
\hline $\begin{array}{l}1937 \\
1938 \\
1939 \\
1940 \\
1941\end{array}$ & $\begin{array}{l}\text { Nov. } 16 \\
\text { Apr. } 11 \\
\text { Mar. } 2 \\
\text { Feb. } 20 \\
\text { Mar. } 26\end{array}$ & $\begin{array}{c}13.4 \\
10.9 \\
16.0 \\
17.3 \\
7.7\end{array}$ & $\begin{array}{r}3,160 \\
1,780 \\
5,300 \\
7,080 \\
812\end{array}$ & $\begin{array}{l}1946 \\
1947 \\
1948 \\
1949 \\
1950\end{array}$ & $\begin{array}{lr}\text { Mar. } & 30 \\
\text { Mar. } 10 \\
\text { Apr. } 1 \\
\text { Feb. } 10 \\
\text { Apr. } 2\end{array}$ & $\begin{array}{r}16.5 \\
17.6 \\
19.9 \\
13.4 \\
9.3\end{array}$ & $\begin{array}{r}5,900 \\
7,900 \\
12,600 \\
2,860 \\
1,120\end{array}$ \\
\hline $\begin{array}{l}1941 \\
1942 \\
1943 \\
1944 \\
1945\end{array}$ & $\begin{array}{lr}\text { Mar. } & 26 \\
\text { Mar. } & 24 \\
\text { Mar. } 10 \\
\text { Mar. } 9 \\
\text { Apr. } 30 \\
\end{array}$ & $\begin{array}{r}7.7 \\
14.4 \\
13.0 \\
17.4 \\
11.0\end{array}$ & $\begin{array}{r}812 \\
3,780 \\
2,810 \\
7,240 \\
1,840 \\
\end{array}$ & $\begin{array}{l}1951 \\
1952 \\
1953\end{array}$ & $\begin{array}{ll}\text { Dec. } 29 \\
\text { Feb. } 19 \\
\text { Dec. } 27\end{array}$ & $\begin{array}{r}8.5 \\
12.4 \\
12.7\end{array}$ & $\begin{array}{r}925 \\
2,310 \\
2,460\end{array}$ \\
\hline
\end{tabular}




\section{Apalachicola River Basin}

(125) Apalachicola River at Chattahoochee, Fla.

'Location.--Lat $30^{\circ} 42103^{\prime \prime}$, long $84^{\circ} 51^{\prime} 33^{\prime \prime}$, in sec. $32, \mathrm{~T}, 4 \mathrm{~N}$, , R. $6 \mathrm{~W}$. , near center of span on downstream slde of bridge on U. S. Highway $90,0.8$ mile downstream from confluence of Flint and Chattahoochee RIvers, and $I$ mile west of Chattahoochee.

Dra1nage area.--17,100 sq $\mathrm{ml}$, approximately.

Records avallable.--U. S. Weather Bureau: October 1919 to December 1928, dally gage helghts. U. S. Geological Survey: December 1928 to December 1953, dally gage helghts and discharges. Records prior to October 1939, publ1shed as "near River Junction".

Gage.--Wire-weight gage read twlce da1ly. Datum of gage 1 s $45.58 \mathrm{ft}$ above mean sea level, datum of 1929 (U. S. Weather Bureau benchmark). Prior to Dec. 11, 1928, U. S. Weather Bureau staff gage read once da1ly and Dec. 11, 1928, to Dec. 15, 1939, water-stage recorder at s1te sevenelghths of a m1le downstream, at datum $0.73 \mathrm{ft}$ lower. Dec. 16, 1939, to June 25, 1952, waterstage recorder at present site and datum.

Stage-discharge relation.--Defined by current-meter measurements below 200,000 cfs. Maximum annual discharges prior to 1929 computed from average rating curve used during per1od 1929-39.

Annual peak stages and discharges

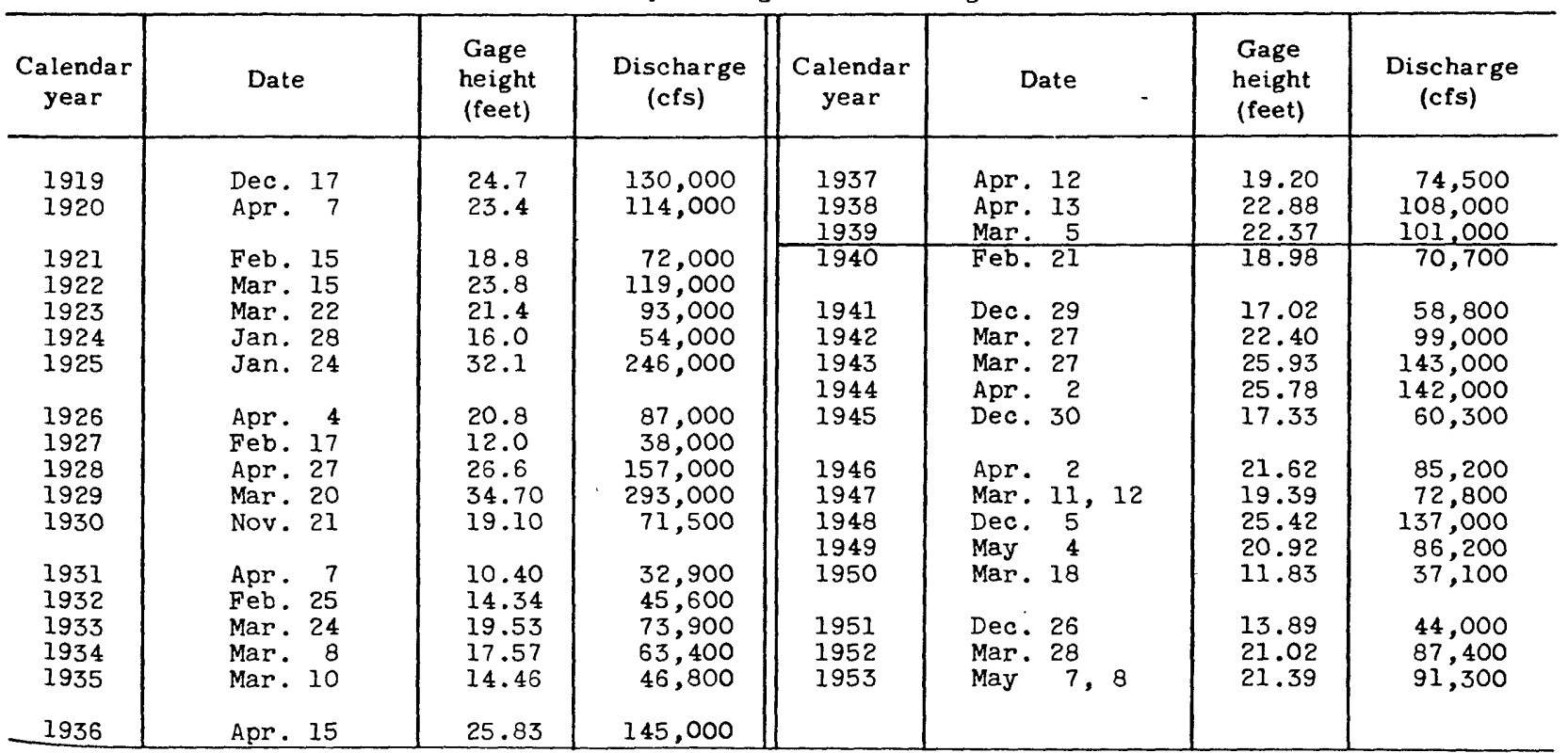

(126) Mosqu1to Creek at Chattahoochee, Fla.

Location.--Lat $30^{\circ} 43^{\prime}$, long $84^{\circ} 49^{\prime}$, in sec. 34, T. 4 N., R. 6 W., at Chattahoochee, $500 \mathrm{ft}$ upstream from bridge on U. S. Highway $90,600 \mathrm{ft}$ downstream from pumping plant and dam, and 3 miles upstream from mouth.

Drainage area.--60 sq $\mathrm{ml}$, approximately.

Records ava1lable.--U. S. Geologlcal Survey: March 1936 to August 1942, da1ly gage helghts and discharges.

Gage.-Water-stage recorder. Datum of gage was not determined.

Stage-discharge relation.--Defined by current-meter measurements below 600 cfs.

Remarks.--About 3 cfs diverted by pumping from reservolr for Florida State Hospital. Some reguiation from changes in reservolr pool. 
Apalachicola R1ver Basin

(126) Mosqu1to Creek at Chattahoochee, Fla.--Continued

Annual peak stages and discharges

\begin{tabular}{|c|c|c|c|c|c|c|c|}
\hline $\begin{array}{c}\text { Calendar } \\
\text { year }\end{array}$ & Date & $\begin{array}{l}\text { Gage } \\
\text { height } \\
\text { (feet) }\end{array}$ & $\begin{array}{c}\text { Discharge } \\
\text { (cfs) }\end{array}$ & $\begin{array}{c}\text { Calendar } \\
\text { year }\end{array}$ & Date & $\begin{array}{c}\text { Gage } \\
\text { height } \\
\text { (feet) }\end{array}$ & $\begin{array}{c}\text { Dischargo } \\
\text { (cfs) }\end{array}$ \\
\hline $\begin{array}{l}1937 \\
1938 \\
1939 \\
\end{array}$ & $\begin{array}{l}\text { Sept. } 1 \\
\text { Mar. } 18 \\
\text { June } 1\end{array}$ & $\begin{array}{r}11.54 \\
5.33 \\
6.82 \\
\end{array}$ & $\begin{array}{r}1,310 \\
296 \\
492 \\
\end{array}$ & $\begin{array}{l}1940 \\
1941 \\
1942 \\
\end{array}$ & $\begin{array}{lr}\text { Feb. } 18 \\
\text { Mar. } 7 \\
\text { Jan. } \quad 4 \\
\end{array}$ & $\begin{array}{r}8.48 \\
5.47 \\
10.99 \\
\end{array}$ & $\begin{array}{r}765 \\
320 \\
1,200 \\
\end{array}$ \\
\hline
\end{tabular}

(127) Apalachicola R1ver near Blountstown, Fla.

Location.--Lat $30^{\circ} 25^{\prime} 25^{\prime \prime}$, long $85^{\circ} 01$ '55", in sec. 3, T. I S., R. 8 W., on right bank at old ferry landing near office and m1ll of Neal Lumber Co. at McNeal, half a m1le upstream from old R1ver Cutoff, and about $1 \frac{1}{2}$ miles southeast of Blountstown.

Drainage area. $--17,300 \mathrm{sq} \mathrm{m1}$, approx1mately.

Records ava1lable.--U. S. Weather Bureau: January 1920 to December 1953, da1ly gage he1ghts. Gage.--Staff gage read once da1ly. Datum of gage $1826.96 \mathrm{ft}$ above mean sea level, datum of 1929.

Stage-d1scharge relation.--Not defined.

H1stor1cal data.--Flood of July 1916 reached a stage of $23.8 \mathrm{ft}$, from Weather Bureau records.

Annual peak stages

\begin{tabular}{|c|c|c|c|c|c|}
\hline Calendar year & Date & $\begin{array}{l}\text { Gage he1ght } \\
\text { (feet) }\end{array}$ & Calendar year & Date & $\begin{array}{l}\text { Gage he1ght } \\
\text { (feet) }\end{array}$ \\
\hline $\begin{array}{l}1916 \\
1920 \\
1921 \\
1922 \\
1923 \\
1924 \\
1925 \\
1926 \\
1927 \\
1928 \\
1929 \\
1930 \\
1931 \\
1932 \\
1933 \\
1934 \\
1935 \\
\end{array}$ & $\begin{array}{l}\text { July }- \\
\text { Apr. } 8 \\
\text { Feb. } 15,16 \\
\text { Mar: } 16 \\
\text { Mar: } 22 \\
\text { Jan. } 29 \\
\text { Jan. } 27 \\
\text { Apr. } 5 \\
\text { Feb: } 19,20 \\
\text { Apr. } 28 \\
\text { Mar: } 21 \\
\text { Nov. } 22 \\
\text { Apr. } 8 \\
\text { Feb: } 26 \\
\text { Mar. } 25 \\
\text { Mar. } 9,10 \\
\text { Mar. } 11,17 \\
\end{array}$ & $\begin{array}{l}23.8 \\
17.6 \\
15.5 \\
22.2 \\
21.0 \\
19.2 \\
27.9 \\
21.7 \\
16.5 \\
24.6 \\
28.6 \\
20.9 \\
14.9 \\
17.8 \\
21.0 \\
20.0 \\
17.8 \\
\end{array}$ & $\begin{array}{l}1936 \\
1937 \\
1938 \\
1939 \\
1940 \\
1941 \\
1942 \\
1943 \\
1944 \\
1945 \\
1946 \\
1947 \\
1948 \\
1949 \\
1950 \\
1951 \\
1952 \\
1953 \\
\end{array}$ & $\begin{array}{l}\text { Apr. } 15 \\
\text { Apr: } 13 \\
\text { Apr: } 14 \\
\text { Mar: } 6 \\
\text { Feb. } 22 \\
\text { Dec. } 30 \\
\text { Mar: } 27,28 \\
\text { Mar. } 27 \\
\text { Apr. } 3 \\
\text { Dec. } 31 \\
\text { Apr. } 3 \\
\text { Mar. } 13,18 \\
\text { Dec. } 6 \\
\text { May } 5 \\
\text { Mar. } 19 \\
\text { Dec. } 28 \\
\text { Mar. } 29 \\
\text { May } 8 \\
\end{array}$ & $\begin{array}{l}23.6 \\
20.7 \\
22.2 \\
22.1 \\
20.9 \\
19.4 \\
22.1 \\
23.5 \\
23.6 \\
20.0 \\
21.7 \\
20.7 \\
23.5 \\
21.5 \\
16.3 \\
17.8 \\
21.5 \\
21.9 \\
\end{array}$ \\
\hline
\end{tabular}


Apalachicola River Basin

(128) Chipola River near Altha, Fla.

Location.--Lat $30^{\circ} 32^{\prime} 02^{\prime \prime}$, long $85^{\circ} 09^{\prime} 55^{\prime \prime}$, in NW $\frac{1}{4} \mathrm{sec} .32$, T. 2 N., R. 9 W., on right bank on downstream side of bridge on State Highway $274,0.9$ mile downstream from Holliman Branch, and $3 \frac{1}{2}$ mlles southwest of Altha.

Dra1nage area. $--781 \mathrm{sq} \mathrm{m}$.

Records ava1lable.--U.S. Geologlcal Survey: November 1912 to December 1913, September 1921 to September 1927, August 1929 to September 1931, March 1943 to December 1953, da1ly gage helghts and discharges.

Gage.-Water-stage recorder. Datum of gage is $19.95 \mathrm{ft}$ above mean sea level (levels by Corps of Englneers). Prior to Jan. 13, 1950, staff, cha1n, or w1re-weight gage at same s1te and datum.

Stage-discharge relation.--Defined by current-meter measurements below 10,000 cfs and by slopearea measurement at 25,000 cfs.

Remarks.--D1scharges for 1913, 1922-25 rev1sed for th1s report.

Annual peak stages and discharges

\begin{tabular}{|c|c|c|c|c|c|c|c|}
\hline $\begin{array}{c}\text { Calendar } \\
\text { year }\end{array}$ & Date & $\begin{array}{c}\text { Gage } \\
\text { height } \\
\text { (feet) }\end{array}$ & $\begin{array}{l}\text { Discharge } \\
\text { (cfs) }\end{array}$ & $\begin{array}{c}\text { Calendar } \\
\text { year }\end{array}$ & Date & $\begin{array}{c}\text { Gage } \\
\text { height } \\
\text { (feet) }\end{array}$ & $\begin{array}{l}\text { Discharge } \\
\text { (cfs) }\end{array}$ \\
\hline 1913 & Mar. 22 & 21.1 & 5,650 & $\begin{array}{l}1943 \\
1944\end{array}$ & $\begin{array}{ll}\text { Mar. } & 12 \\
\text { Mar. } & 28\end{array}$ & $\begin{array}{l}14.58 \\
19.18\end{array}$ & $\begin{array}{l}2,920 \\
4,780\end{array}$ \\
\hline $\begin{array}{l}1922 \\
1923\end{array}$ & $\begin{array}{lr}\text { June } & 2 \\
\text { June } & 29\end{array}$ & $\begin{array}{l}15.98 \\
20.7\end{array}$ & $\begin{array}{l}3,620 \\
5,460\end{array}$ & 1945 & Dec: 31 & 17.55 & 4,040 \\
\hline $\begin{array}{l}1924 \\
1925\end{array}$ & $\begin{array}{l}\text { Sept. } 16 \\
\text { Jan. } 24\end{array}$ & $\begin{array}{l}17.6 \\
23.7\end{array}$ & $\begin{array}{l}4,200 \\
7,010\end{array}$ & $\begin{array}{l}1946 \\
1947\end{array}$ & $\begin{array}{ll}\text { May } & 22 \\
\text { Mar. } & 13\end{array}$ & $\begin{array}{l}23.85 \\
26.42\end{array}$ & $\begin{array}{l}7,070 \\
9,080\end{array}$ \\
\hline 1926 & Sept. 20 & 33.55 & 25,000 & $\begin{array}{l}1948 \\
1949 \\
1950\end{array}$ & $\begin{array}{ll}\text { Apr. } & 4,5 \\
\text { Feb } & 7 \\
\text { Sept. } & 1\end{array}$ & $\begin{array}{l}32.2 \\
17.02 \\
22.5\end{array}$ & $\begin{array}{r}19,100 \\
3,810 \\
6,350\end{array}$ \\
\hline 1929 & oct. & 25.20 & 7,980 & & & & \\
\hline 1930 & Nov. & 19.44 & 4,880 & $\begin{array}{l}1951 \\
1952\end{array}$ & $\begin{array}{ll}\text { Dec. } & 27 \\
\text { Feb. } & 21\end{array}$ & $\begin{array}{l}12.20 \\
17.93\end{array}$ & $\begin{array}{l}2,060 \\
4,180\end{array}$ \\
\hline 1931 & Aug. $\quad 17$ & 17.27 & 3,950 & 1953 & Apr.. 13 & 19.12 & 4,720 \\
\hline
\end{tabular}




\section{Bear Creek Basin}

(129) Econfina Creek near Bennett, Fla.

Location.--Lat $30^{\circ} 23^{\prime} 04^{\prime \prime}$, long $85^{\circ} 33^{\prime} 24^{\prime \prime}$, in sec. 20 , T. I S., R. 13 W., near left bank on downstream slde of bridge on State Highway $388,0.5$ mile downstream from Old Mill Branch, 1.6 miles southwest of Bennett.

Drainage area.--182 sq $\mathrm{ml}$.

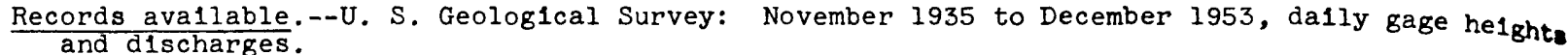

Gage.--Staff gage read once dally. Datum of gage is $1.03 \mathrm{ft}$ above mean sea level, datum of 1929. Stage-discharge relation.--Defined by current-meter measurements below 2,200 cfs.

H1storlcal data.--Maximum stage known, $15.0 \mathrm{ft}$, from floodmarks, e1ther in September 1926 or April 1928 (based on a study of rainfail records).

Annual peak stages and discharges

\begin{tabular}{|c|c|c|c|c|c|c|c|}
\hline $\begin{array}{c}\text { Calendar } \\
\text { year }\end{array}$ & Date & $\begin{array}{l}\text { Gage } \\
\text { height } \\
\text { (feet) }\end{array}$ & $\begin{array}{c}\text { Discharge } \\
\text { (cfs) }\end{array}$ & $\begin{array}{c}\text { Calendar } \\
\text { year }\end{array}$ & Date & $\begin{array}{l}\text { Gage } \\
\text { height } \\
\text { (feet) }\end{array}$ & $\begin{array}{c}\text { Discharge } \\
\text { (cfs) }\end{array}$ \\
\hline $\begin{array}{l}1936 \\
1937 \\
1938 \\
1939 \\
1940\end{array}$ & $\begin{array}{lr}\text { Aug. } & 2 \\
\text { Sept. } & 2 \\
\text { July } & 28 \\
\text { Aug. } & 15 \\
\text { Feb. } & 18\end{array}$ & $\begin{array}{r}9.60 \\
11.00 \\
6.84 \\
10.50 \\
8.46\end{array}$ & $\begin{array}{r}1,310 \\
2,670 \\
708 \\
2,200 \\
1,210\end{array}$ & $\begin{array}{l}1946 \\
1947 \\
1948 \\
1949 \\
1950\end{array}$ & $\begin{array}{lr}\text { May } & 18 \\
\text { Apr. } & 16 \\
\text { Apr. } & 2 \\
\text { Aug. } & 15 \\
\text { Sept. } & 1\end{array}$ & $\begin{array}{r}9.96 \\
9.44 \\
12.46 \\
10.30 \\
10.34\end{array}$ & $\begin{array}{l}1,820 \\
1,530 \\
4,860 \\
2,050 \\
2,050\end{array}$ \\
\hline $\begin{array}{l}1941 \\
1942 \\
1943 \\
1944 \\
1945\end{array}$ & $\begin{array}{lr}\text { Mar. } & 9 \\
\text { Jan. } & 5 \\
\text { May } & 26 \\
\text { Sept. } & 12 \\
\text { Sept. } & 15\end{array}$ & $\begin{array}{r}8.29 \\
8.16 \\
7.80 \\
10.60 \\
9.22\end{array}$ & $\begin{array}{l}1,150 \\
1,120 \\
1,000 \\
2,280 \\
1,440\end{array}$ & $\begin{array}{l}1951 \\
1952 \\
1953\end{array}$ & $\begin{array}{ll}\text { Mar. } 20 \\
\text { Feb. } 18 \\
\text { Sept. } 22,23 \\
\text { Sept. } 26\end{array}$ & $\begin{array}{c}6.90 \\
7.44 \\
- \\
9.47\end{array}$ & $\begin{array}{r}850 \\
\overline{9} \\
943 \\
1,560\end{array}$ \\
\hline
\end{tabular}

\section{Choctawhatchee R1ver Basin}

(130) Choctawhatchee River near Newton, Ala.

Location.--Lat $31^{\circ} 21^{\prime}$, long $85^{\circ} 37^{\prime}$, in SE $\frac{1}{4}$ sec. 2, T. 4 N., R. 24 E., on left bank at downstream s1de of bridge on U. S. H1ghway 231 , $200 \mathrm{ft}$ downstream from mill dam, 1,500 ft upstream from Hurricane Creek, 0.8 mile north of Newton, and 1 mile downstream from Atlantic Coast Line Rallroad bridge.

Dra1nage area. $--683 \mathrm{sq} \mathrm{m1}$.

Records avallable.--U. S. Geological Survey: June 1906 to August 1908, October 1911 to August 1912 , da11y gage heights. November 1921 to September 1927, May 1935 to December 1953, da1ly gage heights and discharges. U. S. Weather Bureau: January 1931 to December 1953, da11y gage helghts.

Gage.--Water-stage recorder. Datum of gage is $138.56 \mathrm{ft}$ above mean sea level, datum of 1929 , supplementary adjustment of 1943. Non-recording gage June 1906 to August 1908, October 1911 to August 1912, and recording gage from November 1921 to Sept. 30, 1927, at s1te $800 \mathrm{ft}$ upstream, at different datum. Non-recording gage at present site from May 10, 1935, to Sept. 8, 1938, and recording gage thereafter.

Stage-d1scharge relation.--Defined by current-meter measurements below $20,000 \mathrm{cfs}$.

H1storlcal data.--Flood of Mar. 15, 1929, reached a stage of $45 \mathrm{ft}$, from information by local residents. (Th1s information 1 s of doubtful accuracy and could not be substantiated in 1954).

Remarks.--Gage-helght records since 1931 are contalned in reports of U. S. Weather Bureau. Peak stage for Nov. 19, 1930, was computed on bas1s of U. S. Weather Bureau gage helghts at Geneva and a stage-relation curve. 
Choctawhatchee R1ver Basin

(130) Choctawhatchee River near Newton, Ala.--Continued

Annual peak stages and discharges

\begin{tabular}{|c|c|c|c|c|c|c|c|}
\hline $\begin{array}{c}\text { Calendar } \\
\text { year }\end{array}$ & Date & $\begin{array}{c}\text { Gage } \\
\text { height } \\
\text { (feet) }\end{array}$ & $\begin{array}{l}\text { Discharge } \\
\text { (cfs) }\end{array}$ & $\begin{array}{c}\text { Calendar } \\
\text { year }\end{array}$ & Date & $\begin{array}{c}\text { Gage } \\
\text { height } \\
\text { (feet) }\end{array}$ & $\begin{array}{c}\text { Discharge } \\
\text { (cfs) }\end{array}$ \\
\hline $\begin{array}{l}1922 \\
1923 \\
1924 \\
1925\end{array}$ & $\begin{array}{ll}\text { June } & \\
\text { Mar. } & 2 \\
\text { Jan. } & 2 \\
\text { Jan. } & 1\end{array}$ & $\begin{array}{r}16.6 \\
18.5 \\
10.6 \\
\times 28.0\end{array}$ & $\begin{array}{r}11,400 \\
13,200 \\
7,340 \\
a 28,000\end{array}$ & $\begin{array}{l}1938 \\
1939 \\
1940\end{array}$ & $\begin{array}{lr}\text { July } 28 \\
\text { Mar. } 1 \\
\text { Feb. } 19\end{array}$ & $\begin{array}{l}18.5 \\
19.7 \\
20.0\end{array}$ & $\begin{array}{l}8,760 \\
9,430 \\
9,600\end{array}$ \\
\hline $\begin{array}{l}1926 \\
1927 \\
\end{array}$ & $\begin{array}{l}\text { Sept. } 2 \\
\text { Feb. } 1\end{array}$ & $\begin{array}{r}23.2 \\
6.4 \\
\end{array}$ & $\begin{array}{r}14,700 \\
3,760 \\
\end{array}$ & $\begin{array}{l}1941 \\
1942 \\
1943\end{array}$ & $\begin{array}{ll}\text { Dec. } 26 \\
\text { Feb. } 17 \\
\text { Jan. } 20\end{array}$ & $\begin{array}{l}10.8 \\
10.0 \\
27.4\end{array}$ & $\begin{array}{r}4,710 \\
4,280 \\
19,300\end{array}$ \\
\hline $\begin{array}{l}1929 \\
1930\end{array}$ & $\begin{array}{l}\text { Mar. } \\
\text { Nov. }\end{array}$ & $\begin{array}{l}45 \\
15.6\end{array}$ & $6, \overline{980}$ & $\begin{array}{l}1944 \\
1945\end{array}$ & $\begin{array}{l}\text { Apr. } 16 \\
\text { Dec. } 25\end{array}$ & $\begin{array}{l}23.4 \\
13.1\end{array}$ & $\begin{array}{r}12,500 \\
5,000\end{array}$ \\
\hline $\begin{array}{l}1931 \\
1932 \\
1933 \\
1934 \\
1935\end{array}$ & $\begin{array}{l}\text { Jan. } \\
\text { May } \\
\text { Mar. } \\
\text { Mar. } \\
\text { July }\end{array}$ & $\begin{array}{r}7.2 \\
9.8 \\
23.8 \\
12.6 \\
12.4\end{array}$ & $\begin{array}{r}2,430 \\
3,810 \\
12,800 \\
5,330 \\
5,220\end{array}$ & $\begin{array}{l}1946 \\
1947 \\
1948 \\
1949 \\
1950\end{array}$ & $\begin{array}{lr}\text { Mar. } & 29 \\
\text { Mar. } & 9 \\
\text { Mar. } & 8 \\
\text { Jan. } & 7 \\
\text { Apr. } & 5\end{array}$ & $\begin{array}{l}25.0 \\
23.7 \\
21.8 \\
18.5 \\
10.7\end{array}$ & $\begin{array}{r}14,600 \\
12,800 \\
10,700 \\
8,610 \\
4,390\end{array}$ \\
\hline $\begin{array}{l}1936 \\
1937 \\
\end{array}$ & $\begin{array}{l}\text { Jan. } \\
\text { Sept. }\end{array}$ & $\begin{array}{l}29.5 \\
26.4 \\
\end{array}$ & $\begin{array}{l}25,800 \\
16,200 \\
\end{array}$ & $\begin{array}{l}1951 \\
1952 \\
1953 \\
\end{array}$ & $\begin{array}{lr}\text { Mar. } 29 \\
\text { Feb. } 16 \\
\text { May } 4 \\
\end{array}$ & $\begin{array}{l}12.6 \\
12.6 \\
29.6 \\
\end{array}$ & $\begin{array}{r}5,400 \\
5,740 \\
23,900 \\
\end{array}$ \\
\hline
\end{tabular}

${ }^{a}$ May have been higher.

(131) Pea R1ver near Samson, Ala.

Location.--Lat $31^{\circ} 07^{\prime}$, long $86^{\circ} 06^{\prime}$, in sec. 25, T. 2 N., R. 19 E., on right bank at downstream s1de of bridge on State H1ghway $12,500 \mathrm{ft}$ downstream from Boyenton Creek, $1 \frac{3}{4}$ m1les downstream from Loulsvilie and Nashville Ra1lroad bridge, 3 miles west of Samson, and $6 \frac{1}{2}$ m1les upstream from Flat Creek.

Drainage area. $--1,187 \mathrm{sq} \mathrm{m1}$.

Records ava1lable.--U. S. Geological Survey: May 1935 to December 1953, da1ly gage helghts and discharges.

Gage.--Water-stage recorder. Datum of gage $1397.95 \mathrm{ft}$ above mean sea level, datum of 1929 , supplementary adjustment of 1943 (levels by Corps of Englneers). May 9, 1935, to July 24, 1937, w1re-welght gage at present s1te and datum.

Stage-discharge relation.--Defined by current-meter measurements.

Historlcal data.--Flood of Jan. 20, 1925 , reached a stage of $42.0 \mathrm{ft}$, from floodmarks (at s1te $1 \frac{1}{2}$ m1les upstream, at different datum\}. Flood of Mar. 15, 1929, reached a stage of $45.3 \mathrm{ft}$, from floodmarks (present s1te and datum). 
Choctawhatchee River Basin

(131) Pea River near Samson, Ala.--Cont1nued

Annual peak stages and discharges

\begin{tabular}{|c|c|c|c|c|c|c|c|}
\hline $\begin{array}{c}\text { Calendar } \\
\text { year }\end{array}$ & Date & $\begin{array}{l}\text { Gage } \\
\text { height } \\
\text { (feet) }\end{array}$ & $\begin{array}{c}\text { Discharge } \\
\text { (cfs) }\end{array}$ & $\begin{array}{c}\text { Calendar } \\
\text { year }\end{array}$ & Date & $\begin{array}{c}\text { Gage } \\
\text { height } \\
\text { (feet) }\end{array}$ & $\begin{array}{c}\text { Discharge } \\
\text { (cfs) }\end{array}$ \\
\hline 1925 & Jan. 20 & 42.0 & - & \multirow{4}{*}{$\begin{array}{l}1944 \\
1945 \\
1946 \\
1947 \\
1948 \\
1949 \\
1950 \\
1951 \\
1952 \\
1953\end{array}$} & \multirow{3}{*}{$\begin{array}{lr}\text { Mar. } & 26 \\
\text { Dec. } & 26 \\
& \\
\text { May } & 22 \\
\text { Apr. } & 6 \\
\text { Dec. } & 1 \\
\text { May. } & 3 \\
\text { Sept. } & 2\end{array}$} & \multirow{3}{*}{$\begin{array}{l}33.4 \\
22.5 \\
30.5 \\
26.7 \\
29.9 \\
24.0 \\
19.5\end{array}$} & \multirow{2}{*}{$\begin{array}{r}19,500 \\
8,920\end{array}$} \\
\hline 1929 & Mar. 15 & 45.3 & - & & & & \\
\hline $\begin{array}{l}1936 \\
1937 \\
1938 \\
1939 \\
1940\end{array}$ & $\begin{array}{lr}\text { Jan. } & 22 \\
\text { Apr. } & 7 \\
\text { Mar. } & 18 \\
\text { Mar. } & 2 \\
\text { Feb. } & 19\end{array}$ & $\begin{array}{l}37.2 \\
35.9 \\
35.8 \\
28.8 \\
22.8\end{array}$ & $\begin{array}{r}27,800 \\
23,400 \\
23,300 \\
14,200 \\
9,120\end{array}$ & & & & $\begin{array}{r}16,000 \\
12,900 \\
15,700 \\
10,000 \\
7,100\end{array}$ \\
\hline $\begin{array}{l}1941 \\
1942 \\
1943\end{array}$ & $\begin{array}{l}\text { Dec. } 29 \\
\text { Apr. } 11 \\
\text { Jan. } 21\end{array}$ & $\begin{array}{l}15.0 \\
20.2 \\
33.2\end{array}$ & $\begin{array}{r}4,790 \\
7,460 \\
19,200 \\
\end{array}$ & & $\begin{array}{lr}\text { Mar. } & 30 \\
\text { Mar. } & 28 \\
\text { Dec. } & 5\end{array}$ & $\begin{array}{l}19.5 \\
24.7 \\
31.7\end{array}$ & $\begin{array}{r}7,100 \\
10,600 \\
17,400\end{array}$ \\
\hline
\end{tabular}

(132) Choctawhatchee R1ver at Caryville, Fla.

Location.--Lat $30^{\circ} 46^{\prime} 32^{\prime \prime}$, long $85^{\circ} 49^{\prime} 40^{\prime \prime}$, in sec. 10, T. 4 N., R. 16 W., near right bank on downstream side of bridge on U. S. H1ghway $90,300 \mathrm{ft}$ downstream from Loulsville and Nashville Ra1lroad bridge, three-quarters of a mile west of Caryville, and 1.8 mlles downstream from Wrights Creek.

Dra1nage area. $--3,499$ sq $\mathrm{ml}$.

Records ava1lable.--U. S. Weather Bureau: November 1928 to August 1929, da1ly gage helghts. U. S. Geolog1cal Survey: August 1929 to December 1953, da1ly gage helghts and discharges.

Gage.--W1re-we1ght gage read twice dally. Datum of gage is $39.00 \mathrm{ft}$ above mean sea level, datum of 1929. Prior to Oct. 12, 1929, staff gage and Oct. 12, 1929, to Sept. 11, 1951, water-stage recorder at same site and datum.

Stage-discharge relation.--Deflned by current-meter measurements below 50,000 cfs and by slopearea measurement at $206,000 \mathrm{cfs}$.

H1stor1cal data.--Max1mum stage known, $27.1 \mathrm{ft}$ Mar. 17, 1929, from Weather Bureau records and floodmarks. Flood of Apr. 24, 1928, reached a stage of $21.6 \mathrm{ft}$, from Weather Bureau records.

Annual peak stages and discharges

\begin{tabular}{|c|c|c|c|c|c|c|c|}
\hline $\begin{array}{l}\text { Calendar } \\
\text { year }\end{array}$ & Date & $\begin{array}{l}\text { Gage } \\
\text { height } \\
\text { (feet) }\end{array}$ & $\begin{array}{l}\text { Discharge } \\
\text { (cfs) }\end{array}$ & $\begin{array}{c}\text { Calendar } \\
\text { year }\end{array}$ & Date & $\begin{array}{l}\text { Gage } \\
\text { height } \\
\text { (feet) }\end{array}$ & $\begin{array}{l}\text { Discharge } \\
\text { (cfs) }\end{array}$ \\
\hline $\begin{array}{l}1928 \\
1929 \\
1930\end{array}$ & $\begin{array}{ll}\text { Apr: } & 24 \\
\text { Mar: } & 17 \\
\text { Nov. } & 19\end{array}$ & $\begin{array}{l}21.6 \\
27.1 \\
13.17\end{array}$ & $\begin{array}{r}129,000 \\
206,000 \\
31,700\end{array}$ & $\begin{array}{l}1941 \\
1942 \\
1943 \\
1944\end{array}$ & $\begin{array}{lr}\text { Dec. } & 29 \\
\text { Jan. } & 5 \\
\text { Jan. } & 24 \\
\text { Mar. } & 28\end{array}$ & $\begin{array}{l}10.45 \\
11.86 \\
13.42 \\
13.60\end{array}$ & $\begin{array}{l}13,700 \\
21,400 \\
33,800 \\
35,800\end{array}$ \\
\hline $\begin{array}{l}1931 \\
1932\end{array}$ & $\begin{array}{ll}\text { Jan. } & 21 \\
\text { Jan. } & 16\end{array}$ & $\begin{array}{r}9.78 \\
12.19\end{array}$ & $\begin{array}{r}9,780 \\
23,400\end{array}$ & 1945 & Dec. 29 & 12.22 & 23,300 \\
\hline $\begin{array}{l}1933 \\
1934 \\
1935\end{array}$ & $\begin{array}{lr}\text { Mar: } & 24 \\
\text { Mar. } & 8 \\
\text { Mar. } & 12\end{array}$ & $\begin{array}{l}13.58 \\
12.28 \\
10.17\end{array}$ & $\begin{array}{l}35,800 \\
24,000 \\
12,600\end{array}$ & $\begin{array}{l}1946 \\
1947 \\
1948\end{array}$ & $\begin{array}{ll}\text { Mar. } & 31 \\
\text { Mar. } & 10 \\
\text { Mar. } & 10\end{array}$ & $\begin{array}{l}14.03 \\
14.46 \\
14.47\end{array}$ & $\begin{array}{l}39,800 \\
44,800 \\
44,800\end{array}$ \\
\hline $\begin{array}{l}1936 \\
1937\end{array}$ & $\begin{array}{lr}\text { Jan. } & 24 \\
\text { Sept. } & 4\end{array}$ & $\begin{array}{l}14.16 \\
15.55\end{array}$ & $\begin{array}{l}41,800 \\
56,600\end{array}$ & $\begin{array}{l}1949 \\
1950\end{array}$ & $\begin{array}{ll}\text { Jan. } & 9 \\
\text { Sept. } & 3\end{array}$ & $\begin{array}{l}12.46 \\
12.44\end{array}$ & $\begin{array}{l}25,300 \\
25,100\end{array}$ \\
\hline $\begin{array}{l}1938 \\
1939 \\
1940\end{array}$ & $\begin{array}{ll}\text { Mar. } & 22 \\
\text { Aug. } & 18 \\
\text { Feb. } & 21,22\end{array}$ & $\begin{array}{l}13.03 \\
14.84 \\
12.90\end{array}$ & $\begin{array}{l}30,100 \\
45,800 \\
29,200\end{array}$ & $\begin{array}{l}1951 \\
1952 \\
1953\end{array}$ & $\begin{array}{l}\text { Apr. } \\
\text { Feb: } \quad 19,20 \\
\text { Dec. }\end{array}$ & $\begin{array}{l}11.14 \\
11.52 \\
15.50\end{array}$ & $\begin{array}{l}16,500 \\
17,900 \\
54,800\end{array}$ \\
\hline
\end{tabular}


Location.--Lat $30^{\circ} 27^{\prime} 03^{\prime \prime}$, long $85^{\circ} 53^{\prime} 54^{\prime \prime}$, in sec. 36, T. I N., R. 17 W., on downstream fender pile at center swing pier of bridge on State Highway 20 , about 4 miles southeast of Bruce, and 5.8 miles downstream from Holmes Creek.

Drainage area. $--4,384 \mathrm{sq} \mathrm{ml}$.

Records avallable.--U. S. Geological Survey: October 1930 to December 1953, da1ly gage heights and discharges.

Gage.-Water-stage recorder. Datum of gage is $3.94 \mathrm{ft}$ above mean sea level, datum of 1929. Prior to Apr. 6, 1934, staff gage at site 1 mile downstream at datum 0.25 ft lower.

Stage-discharge relation.--Defined by current-meter measurements below 66,000 cfs and extended above on basis of records for station at Caryville.

Historical data.--Maximum stage known about $25.0 \mathrm{ft}$, from floodmarks at former site and datum, in March 1929.

Annual peak stages and discharges

\begin{tabular}{|c|c|c|c|c|c|c|c|}
\hline $\begin{array}{c}\text { Calendar } \\
\text { year }\end{array}$ & Date & $\begin{array}{c}\text { Gage } \\
\text { height } \\
\text { (feet) }\end{array}$ & $\begin{array}{l}\text { Discharge } \\
\text { (cfs) }\end{array}$ & $\begin{array}{c}\text { Calendar } \\
\text { year }\end{array}$ & Date & $\begin{array}{l}\text { Gage } \\
\text { height } \\
\text { (feet) }\end{array}$ & $\begin{array}{l}\text { Discharge } \\
\text { (cfs) }\end{array}$ \\
\hline $\begin{array}{l}1929 \\
1930\end{array}$ & Mar: $\overline{22}$ & $\begin{array}{l}25.0 \\
12.25\end{array}$ & $\begin{array}{r}220,000 \\
39,100\end{array}$ & \multirow{8}{*}{$\begin{array}{l}1942 \\
1943 \\
1944 \\
1945 \\
\\
1946 \\
1947 \\
1948 \\
1949 \\
1950 \\
1951 \\
1952 \\
1953\end{array}$} & \multirow{8}{*}{$\begin{array}{lr}\text { Jan. } & 5 \\
\text { Jan. } & 26 \\
\text { Mar. } & 30 \\
\text { Dec. } & 31 \\
& \\
\text { Aug. } & 10 \\
\text { Mar. } & 12 \\
\text { Apr. } & 4 \\
\text { May } & 6 \\
\text { Sept. } & 5 \\
& \\
\text { Apr. } & 5 \\
\text { Feb. } & 22 \\
\text { Dec. } & 12\end{array}$} & \multirow{8}{*}{$\begin{array}{r}12.45 \\
12.13 \\
12.90 \\
10.81 \\
13.88 \\
14.79 \\
15.22 \\
10.97 \\
12.15 \\
8.93 \\
10.16 \\
13.82\end{array}$} & \multirow{8}{*}{$\begin{array}{l}35,200 \\
33,000 \\
39,200 \\
24,500 \\
47,200 \\
54,400 \\
57,600 \\
25,800 \\
33,400\end{array}$} \\
\hline $\begin{array}{l}1931 \\
1932\end{array}$ & $\begin{array}{ll}\text { Jan. } & 24 \\
\text { Jan. } & 19\end{array}$ & $\begin{array}{r}7.80 \\
10.30\end{array}$ & $\begin{array}{l}12,100 \\
25,100\end{array}$ & & & & \\
\hline $\begin{array}{l}1933 \\
1934\end{array}$ & $\begin{array}{ll}\text { Apr. } & 18 \\
\text { Mar. } & 11\end{array}$ & $\begin{array}{l}12.40 \\
10.58\end{array}$ & $\begin{array}{l}40,700 \\
27,200\end{array}$ & & & & \\
\hline 1935 & Mar. 16 & 8.23 & 13,600 & & & & \\
\hline $\begin{array}{l}1936 \\
1937\end{array}$ & $\begin{array}{l}\text { Jan. } 10 \\
\text { Sept. } 6\end{array}$ & $\begin{array}{l}13.96 \\
16.04\end{array}$ & $\begin{array}{l}49,500 \\
64,000\end{array}$ & & & & \\
\hline 1938 & Mar. 24 & 11.78 & 30,900 & & & & \\
\hline $\begin{array}{l}1939 \\
1940\end{array}$ & $\begin{array}{ll}\text { Aug. } & 19 \\
\text { Feb. } & 23\end{array}$ & $\begin{array}{l}16.68 \\
12.07\end{array}$ & $\begin{array}{l}69,600 \\
33,000\end{array}$ & & & & \\
\hline 1941 & Mar. 27 & 7.78 & 12,400 & & & & \\
\hline
\end{tabular}

\section{Yellow River Basin}

(134) Lightwood Knot Creek at Babble, Ala.

Location.--Lat $31^{\circ} 16^{\prime}$, long $86^{\circ} 19^{\prime}$, in SW $\frac{1}{4}$ sec. 36, T. $4 \mathrm{~N}$. R. $17 \mathrm{E}$., on downstream side of right pier on U. S. Highway 84,1 mile east of Babble, $1 \frac{1}{4}$ miles downstream from Poley Creek, 2 miles upstream from mouth, and $3 \frac{1}{2}$ miles west of Opp.

Dra1nage area. --113 sq $\mathrm{ml}$.

Records ava1lable.--U. S. Geological Survey: February 1944 to Apr11 1953, da1ly gage heights and discharges. Prior to October 1947, published as "Poley Creek".

Gage.--Water-stage recorder. Prior to Dec. 30, 1947, wire-weight gage at same site and datum. Stage-discharge relation.--Defined by current-meter measurements below $9,000 \mathrm{cfs}$. 
Yellow River Basin

(134) Lightwood Knot Creek at Babbie, Ala.--Continued

Annual peak stages and discharges

\begin{tabular}{c|c|c|c||c|c|c|c}
\hline $\begin{array}{c}\text { Calendar } \\
\text { year }\end{array}$ & Date & $\begin{array}{c}\text { Gage } \\
\text { height } \\
\text { (feet) }\end{array}$ & $\begin{array}{c}\text { Discharge } \\
\text { (cfs) }\end{array}$ & $\begin{array}{c}\text { Calendar } \\
\text { year }\end{array}$ & Date & $\begin{array}{c}\text { Gage } \\
\text { height } \\
\text { (feet) }\end{array}$ & $\begin{array}{c}\text { Discharge } \\
\text { (cfs) }\end{array}$ \\
\hline 1944 & Sept. 11 & 11.9 & 12,100 & 1949 & Jan. 6 & 7.5 & 3,260 \\
1945 & Dec. 25 & 7.8 & 3,500 & 1950 & Apr. 5 & 6.8 & 2,000 \\
1946 & May 20 & 8.6 & 5,100 & 1951 & Apr. 20 & 7.1 & 1,660 \\
1947 & Apr. 16 & 7.5 & 3,260 & 1952 & Mar. 24 & 7.4 & 2,100 \\
1948 & Apr. 1 & 7.6 & 3,440 & 1953 & May 6 & 9.4 & 6,700 \\
\hline
\end{tabular}

(135) Yellow R1ver at M1ll1gan, Fla.

Locat1on. --Lat $30^{\circ} 45^{\prime} 10^{\prime \prime}$, long $86^{\circ} 37^{\prime} 45^{\prime \prime}$, in sec. 15, T. $3 \mathrm{~N} ., \mathrm{R} .24 \mathrm{~W}$., on right bank $10 \mathrm{ft}$ downstream from bridge on U. S. H1ghway 90 , half a mile east of M1lilgan, half a m1le upstream from Trammel Creek, and $6 \frac{3}{4}$ miles upstream from Shoal River.

Dra1nage area. $--624 \mathrm{sq} \mathrm{ml}$.

Records avallable.--U. S. Geologlcal Survey: July 1938 to December 1953, da1ly gage helghts and discharges.

Gage.--Water-stage recorder. Datum of gage is $51.55 \mathrm{ft}$ above mean sea level, datum of 1929. Prior to Dec. 6, 1939, staff gage at same site and datum.

Stage-d1scharge relation.--Defined by current-meter measurements below 24,000 cfs. Sl1ght shifting at all stages.

H1storical data.--Floods of 1928 and 1929 reached stages of $20.2 \mathrm{ft}$ and $26.2 \mathrm{ft}$, respectively, from information by local residents.

Annual peak stages and discharges

\begin{tabular}{|c|c|c|c|c|c|c|c|}
\hline $\begin{array}{c}\text { Calendar } \\
\text { year }\end{array}$ & Date & $\begin{array}{l}\text { Gage } \\
\text { height } \\
\text { (feet) }\end{array}$ & $\begin{array}{l}\text { Discharge } \\
\text { (cfs) }\end{array}$ & $\begin{array}{c}\text { Calendar } \\
\text { year }\end{array}$ & Date & $\begin{array}{c}\text { Gage } \\
\text { height } \\
\text { (feet) }\end{array}$ & $\begin{array}{c}\text { Discharge } \\
\text { (cfs) }\end{array}$ \\
\hline $\begin{array}{l}1928 \\
1929 \\
1939 \\
1940\end{array}$ & $\begin{array}{ll} & - \\
\text { Mar. } & - \\
\text { Aug. } & 19 \\
\text { July } & 8\end{array}$ & $\begin{array}{l}20.2 \\
26.2 \\
12.44 \\
12.00\end{array}$ & $\begin{array}{c}- \\
- \\
14,700 \\
13,500\end{array}$ & $\begin{array}{l}1946 \\
1947 \\
1948 \\
1949 \\
1950\end{array}$ & $\begin{array}{lr}\text { Mar. } & 30 \\
\text { Mar: } & 10 \\
\text { Mar: } & 9 \\
\text { May } & 3 \\
\text { Sept. } & 2\end{array}$ & $\begin{array}{r}11.13 \\
11.20 \\
11.23 \\
9.12 \\
9.67\end{array}$ & $\begin{array}{r}11,300 \\
11,800 \\
11,800 \\
5,260 \\
7,000\end{array}$ \\
\hline $\begin{array}{l}1941 \\
1942 \\
1943 \\
1944 \\
1945 \\
\end{array}$ & $\begin{array}{ll}\text { Mar. } 23,24 \\
\text { June } 26 \\
\text { Jan. } 22 \\
\text { Sept. } 13 \\
\text { Apr. } 30\end{array}$ & $\begin{array}{r}7.47 \\
9.10 \\
10.80 \\
13.29 \\
8.84\end{array}$ & $\begin{array}{r}2,430 \\
4,800 \\
9,420 \\
21,200 \\
4,140\end{array}$ & $\begin{array}{l}1951 \\
1952 \\
1953\end{array}$ & $\begin{array}{lr}\text { Mar. } & 22 \\
\text { Mar: } & 28 \\
\text { Dec. } & 6\end{array}$ & $\begin{array}{r}8.03 \\
8.85 \\
15.13\end{array}$ & $\begin{array}{r}2,930 \\
4,490 \\
28,000\end{array}$ \\
\hline
\end{tabular}


Yellow R1ver Basin

(136) Shoal River near Crestview, Fla.

Location.--Lat $30^{\circ} 41^{\prime} 50^{\prime \prime}$, long $86^{\circ} 34^{\prime} 15^{\prime \prime}$, in sec. 5, T. 2 N., R. 23 W., on right bank on downstream side of bridge on State Highway $85,3 \frac{1}{2}$ miles downstream from Tit1 Creek, $4 \frac{1}{4}$ miles south of Crestview, and 7 miles upstream from mouth.

Drainage area. $--474 \mathrm{sq} \mathrm{ml}$.

Records avallable.--U. S. Geological Survey: July 1938 to December 1953, da1ly gage heights and discharges.

Gage.--Water-stage recorder. Datum of gage is $47.21 \mathrm{ft}$ above mean sea level, datum of 1929. Prior to Feb. 12, 1939, staff gage at same site and datum.

Stage-discharge relation.--Defined by current-meter measurements. Silght shifting at medium and low stages.

Historical data.--Flood of March 1929 reached a stage of about $17 \mathrm{ft}$, from floodmarks.

Annual peak stages and discharges

\begin{tabular}{|c|c|c|c|c|c|c|c|}
\hline $\begin{array}{l}\text { Calendar } \\
\text { year }\end{array}$ & Date & $\begin{array}{l}\text { Gage } \\
\text { height } \\
\text { (feet) }\end{array}$ & $\begin{array}{l}\text { Discharge } \\
\text { (cfs) }\end{array}$ & $\begin{array}{l}\text { Calendar } \\
\text { year }\end{array}$ & Date & $\begin{array}{c}\text { Gage } \\
\text { height } \\
\text { (feet) }\end{array}$ & $\begin{array}{l}\text { Discharge } \\
\text { (cfs) }\end{array}$ \\
\hline $\begin{array}{l}1929 \\
1939 \\
1940\end{array}$ & $\begin{array}{lr}\text { Mar. } & - \\
\text { Aug. } & 18 \\
\text { July } & 7\end{array}$ & $\begin{array}{c}\text { about } 17 \\
11.18 \\
14.26\end{array}$ & $\begin{array}{c}- \\
13,400 \\
21,700\end{array}$ & $\begin{array}{l}1946 \\
1947 \\
1948 \\
1949 \\
1950\end{array}$ & $\begin{array}{lr}\text { Mar. } & 29 \\
\text { Mar. } & 9 \\
\text { Apr. } & 3 \\
\text { Apr. } & 14 \\
\text { Sept. } & 2\end{array}$ & $\begin{array}{r}9.23 \\
12.57 \\
9.54 \\
7.68 \\
10.38\end{array}$ & $\begin{array}{r}8,030 \\
17,100 \\
8,870 \\
4,650 \\
11,200\end{array}$ \\
\hline $\begin{array}{l}1941 \\
1942 \\
1943 \\
1944 \\
1945 \\
\end{array}$ & $\begin{array}{lr}\text { July } & 7 \\
\text { Jan. } & 3 \\
\text { Mar. } & 8 \\
\text { Sept. } & 12 \\
\text { Jan. } & 8 \\
\end{array}$ & $\begin{array}{r}7.26 \\
8.33 \\
7.15 \\
11.05 \\
7.67 \\
\end{array}$ & $\begin{array}{r}3,700 \\
5,960 \\
3,520 \\
13,000 \\
4,560 \\
\end{array}$ & $\begin{array}{l}1951 \\
1952 \\
1953\end{array}$ & $\begin{array}{ll}\text { Mar. } 20 \\
\text { Feb. } 16,17 \\
\text { Sept. } 28\end{array}$ & $\begin{array}{r}7.56 \\
6.96 \\
13.93\end{array}$ & $\begin{array}{r}4,380 \\
3,120 \\
20,000\end{array}$ \\
\hline
\end{tabular}

(137) Yellow River near Holt, Fla.

Location.--Lat $30^{\circ} 40^{\prime} 25^{\prime \prime}$, long $86^{\circ} 44^{\prime} 50^{\prime \prime}$, in sec. 16, T. 2 N., R. 25 W., at county highway bridge $2 \frac{1}{2}$ miles south of Holt.

Drainage area.--1,220 sq $\mathrm{ml}$, approximately.

Records avallable.--U. S. Geological Surveyr October 1933 to September 194l, daily gage heights and discharges.

Gage.--Staff gage read once da1ly. Datum of gage is $17.95 \mathrm{ft}$ above mean sea level, datum of 1929 .

Stage-discharge relation.--Defined by current-meter measurements below 18,000 cfs. Considerable shifting at all stages.

Historical data.--Flood of March 1929 reached a stage of $25.4 \mathrm{ft}$, from information by local residents. 
Yellow R1ver Basin

(137) Yellow R1ver near Holt, Fla.--Cont1nued

Annual peak stages and discharges

\begin{tabular}{|c|c|c|c|c|c|c|c|}
\hline $\begin{array}{c}\text { Calendar } \\
\text { year }\end{array}$ & Date & $\begin{array}{c}\text { Gage } \\
\text { height } \\
\text { (feet) }\end{array}$ & $\begin{array}{c}\text { Discharge } \\
\text { (cfs) }\end{array}$ & $\begin{array}{c}\text { Calendar } \\
\text { year }\end{array}$ & Date & $\begin{array}{l}\text { Gage } \\
\text { height } \\
\text { (feet) }\end{array}$ & $\begin{array}{c}\text { Dischare } \\
\text { (cts) }\end{array}$ \\
\hline $\begin{array}{l}1929 \\
1934 \\
1935\end{array}$ & $\begin{array}{lr}\text { Mar. } & - \\
\text { Oct. } & 9 \\
\text { Mar. } & 13\end{array}$ & $\begin{array}{r}25.4 \\
12.80 \\
7.20\end{array}$ & $\begin{array}{c}- \\
25,000 \\
4,850\end{array}$ & $\begin{array}{l}1937 \\
1938 \\
1939 \\
1940\end{array}$ & $\begin{array}{lr}\text { Apr. } & 9 \\
\text { Mar. } & 21 \\
\text { Aug. } & 19 \\
\text { July } & 9\end{array}$ & $\begin{array}{r}11.02 \\
8.50 \\
13.90 \\
15.62\end{array}$ & $\begin{array}{r}16,300 \\
7,900 \\
27,800 \\
35,100\end{array}$ \\
\hline 1936 & Jan. 22 & 11.48 & 16,600 & 1941 & July 8 & 7.76 & 6,030 \\
\hline
\end{tabular}

Blackwater River Basin

(138) Coldwater Creek near M1lton, Fla.

Location.--Lat $30^{\circ} 42^{\prime} 30^{\prime \prime}$, long $86^{\circ} 58^{\prime} 20^{\prime \prime}$, In sec. 5, T. 2 N., R. 27 W., on r1ght bank at downstream slde of bridge on State H1ghway 191, $2 \frac{3}{4}$ miles upstream from mouth and $6 \frac{1}{2} \mathrm{~m} 11 \mathrm{es}$ northeast of Milton.

Dra1nage area. $--237 \mathrm{sq} \mathrm{m} 1$.

Records avallable.--U. S. Geologlcal Survey: November 1938 to December 1953, da1ly gage heighte and discharges.

Gage.-Water-stage recorder. Datum of gage 1s $9.10 \mathrm{ft}$ above mean sea level, datum of 1929. Prior to Dec. 2, 1938, staff gage at same site and datum.

Stage-discharge relation.--Def1ned by current-meter measurements below $18,000 \mathrm{cfs}$.

Annual peak stages and discharges

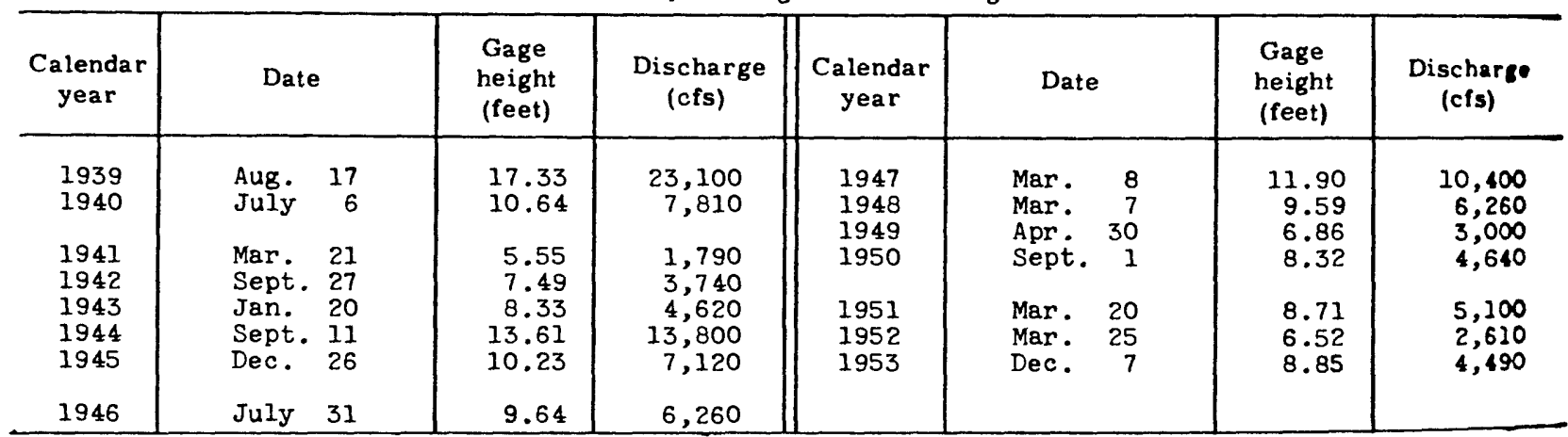


Escambia River Basin

(139) Conecuh River near Andalusia, Ala.

Location.--Lat $31^{\circ} 16^{\prime}$, long $86^{\circ} 36^{\prime}$, in $N E \frac{1}{4} \mathrm{sec} .1, T .3 \mathrm{~N}$., R. $14 \mathrm{E}$, on left bank on downstream slde of Simmons Bridge on State Highway 83, $7 \frac{1}{2} \mathrm{mlles}$ southwest of Andalusia, and $10 \frac{1}{2} \mathrm{mlles}$ downstream from Patsaliga Creek.

Dra1nage area. $--1,343 \mathrm{sq} \mathrm{m} 1$.

Records avallable.--U. S. Geological Survey: August 1904 to December 1919, September 1929 to December 1953, daliy gage helghts and discharges. Records published as "at Beck" 1904-19.

Gage.--Water-stage recorder. Datum of gage is $106.77 \mathrm{ft}$ above mean sea level, datum of 1929 , supplementary adjustment of 1943 (levels by Corps of Engineers). Aug. 21, 1904, to Dec. 31, 1919, chain gage at same site and datum.

Stage-discharge relation.--Defined by current-meter measurements below 37,000 cfs and by slopearea determination at $154,000 \mathrm{cfs}$.

Remarks.--Annual floods listed for 1905-19 are mean da1ly discharges; peak discharges undetermined but probably did not exceed the figures shown by more than io percent.

Annual peak stages and discharges

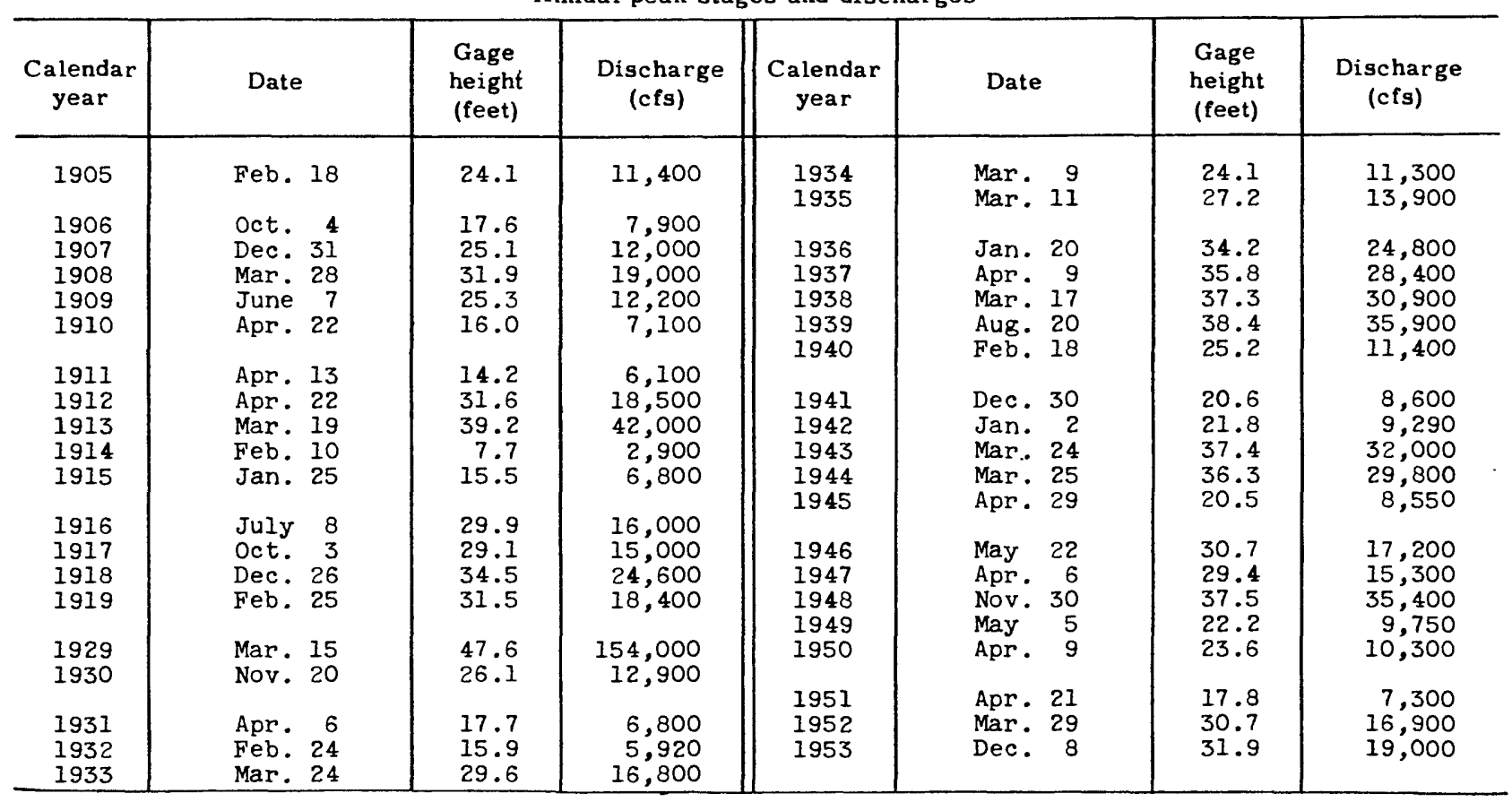


(140) Sepulga River near McKenzle, Ala.

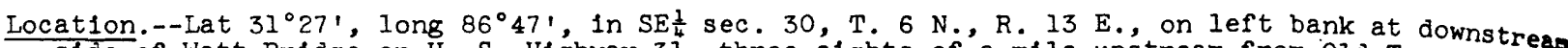
side of Watt Bridge on U. S. Highway 3l, three-elghts of a mile upstream from Old Town Creek.

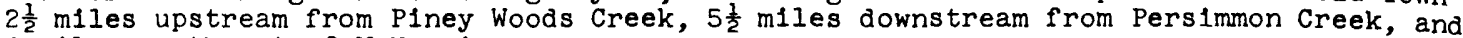

7 miles southwest of McKenzie.

Drainage area. $-464 \mathrm{sq} \mathrm{ml}$.

Records avallable.--U. S. Geological Survey: October 1937 to December 1953, da1ly gage helghts and discharges.

Gage.--Water-stage recorder. Datum of gage is $155.96 \mathrm{ft}$ above mean sea level, unadjusted (levels by Corps of Engineers). Prior to Mar. 25, 1939, wire-weight gage at same site and datum.

Stage-discharge relation.--Defined by current-meter measurements below 25,000 cfs.

H1storical data.--The flood of March 1929 reached a stage of about $33 \mathrm{ft}$, from information by local residents.

Annual peak stages and discharges

\begin{tabular}{|c|c|c|c|c|c|c|c|}
\hline $\begin{array}{c}\text { Calendar } \\
\text { year }\end{array}$ & Date & $\begin{array}{l}\text { Gage } \\
\text { height } \\
\text { (feet) }\end{array}$ & $\begin{array}{l}\text { Discharge } \\
\text { (cfs) }\end{array}$ & $\begin{array}{c}\text { Calendar } \\
\text { year }\end{array}$ & Date & $\begin{array}{c}\text { Gage } \\
\text { height } \\
\text { (feet) }\end{array}$ & $\begin{array}{l}\text { Discharge } \\
\text { (cfs) }\end{array}$ \\
\hline $\begin{array}{l}1929 \\
1938 \\
1939 \\
1940\end{array}$ & $\begin{array}{ll}\text { Mar. } & - \\
& \\
\text { Mar. } 17 \\
\text { Aug. } 18 \\
\text { Feb. } 20\end{array}$ & $\begin{array}{l}33 \\
24.5 \\
19.5 \\
16.0\end{array}$ & $\begin{array}{c}- \\
28,100 \\
13,400 \\
9,000\end{array}$ & $\begin{array}{l}1946 \\
1947 \\
1948 \\
1949 \\
1950\end{array}$ & $\begin{array}{lr}\text { Jan. } & 8 \\
\text { Apr. } & 4 \\
\text { Nov. } & 28 \\
\text { May } & 4 \\
\text { July } & 30\end{array}$ & $\begin{array}{r}21.9 \\
16.5 \\
23.6 \\
11.9 \\
7.7\end{array}$ & $\begin{array}{r}19,400 \\
9,470 \\
21,200 \\
6,120 \\
2,950\end{array}$ \\
\hline $\begin{array}{l}1941 \\
1942 \\
1943 \\
1944 \\
1945\end{array}$ & $\begin{array}{ll}\text { Dec. } & 26 \\
\text { Mar. } 24 \\
\text { Mar. } 23 \\
\text { Mar. } 31 \\
\text { Dec. } 27\end{array}$ & $\begin{array}{r}13.5 \\
11.4 \\
21.8 \\
22.3 \\
8.2\end{array}$ & $\begin{array}{r}7,100 \\
5,750 \\
19,100 \\
20,600 \\
3,290\end{array}$ & $\begin{array}{l}1951 \\
1952 \\
1953\end{array}$ & $\begin{array}{lr}\text { Apr. } & 21 \\
\text { Mar. } & 25 \\
\text { Dec. } & 8\end{array}$ & $\begin{array}{l}12.9 \\
11.6 \\
14.1\end{array}$ & $\begin{array}{l}6,920 \\
5,880 \\
7,940\end{array}$ \\
\hline
\end{tabular}

(141) Pigeon Creek near Thad, Ala.

Location.--Lat $31^{\circ} 29^{\prime}$, long $86^{\circ} 39^{\prime}$, in $\mathrm{N} \frac{1}{2} \mathrm{sec} .21$, T. $6 \mathrm{~N}$., R. 14 E., on left bank near downstream side of bridge on U.'S. H1ghway 84, I li miles upstream from Loulsville and Nashville Rallroad bridge, 2 miles southeast of Thad, 3 miles upstream from Reedy Creek, and $5 \frac{1}{2} \mathrm{miles}$ southeast of McKenzie.

Drainage area. $--296 \mathrm{sq} \mathrm{ml}$.

Records avallable.--U. S. Geological Survey: October 1937 to December 1953, da1ly gage helghts and discharges.

Gage.--Water-stage recorder. Datum of gage is $172.58 \mathrm{ft}$ above mean sea level, datum of 1929 , supplementary adjustment of 1943. Prior to oct. 24, 1938, w1re-welght gage at same site and datum.

Stage-discharge relation.--Defined by current-meter measurements below 14,000 cfs.

Historical data.--The flood of March 1929 reached a stage of about $30 \mathrm{ft}$, from information by local residents. 
Escamb1a R1ver Basin

(141) Pigeon Creek near Thad, Ala.--Continued

Annual peak stages and discharges

\begin{tabular}{|c|c|c|c|c|c|c|c|}
\hline $\begin{array}{c}\text { Calendar } \\
\text { year }\end{array}$ & Date & $\begin{array}{c}\text { Gage } \\
\text { height } \\
\text { (feet) }\end{array}$ & $\begin{array}{c}\text { Discharge } \\
\text { (cfs) }\end{array}$ & $\begin{array}{c}\text { Calendar } \\
\text { year }\end{array}$ & Date & $\begin{array}{l}\text { Gage } \\
\text { height } \\
\text { (feet) }\end{array}$ & $\begin{array}{c}\text { Discharge } \\
\text { (cfs) }\end{array}$ \\
\hline $\begin{array}{l}1929 \\
1938 \\
1939 \\
1940\end{array}$ & $\begin{array}{l}\text { Mar. }- \\
\text { Mar. } 18 \\
\text { Aug. 17, } 18 \\
\text { Feb. } 18\end{array}$ & $\begin{array}{l}30 \\
26.1 \\
25.3 \\
17.6\end{array}$ & $\begin{array}{c}- \\
14,400 \\
12,600 \\
3,740\end{array}$ & $\begin{array}{l}1946 \\
1947 \\
1948 \\
1949 \\
1950\end{array}$ & $\begin{array}{lr}\text { Jan. } & 8 \\
\text { Apr. } & 5 \\
\text { Nov. } & 29 \\
\text { July } & 19 \\
\text { July } & 29\end{array}$ & $\begin{array}{l}25.1 \\
18.5 \\
27.1 \\
16.5 \\
10.1\end{array}$ & $\begin{array}{r}12,600 \\
4,220 \\
17,100 \\
3,270 \\
1,110\end{array}$ \\
\hline $\begin{array}{l}1941 \\
1942 \\
1943 \\
1944 \\
1945\end{array}$ & $\begin{array}{l}\text { Dec. } 27 \\
\text { Mar. } 25 \\
\text { Mar. } 23 \\
\text { Apr. } 28 \\
\text { Dec. } 30\end{array}$ & $\begin{array}{l}20.6 \\
15.9 \\
25.2 \\
25.0 \\
13.1\end{array}$ & $\begin{array}{r}5,730 \\
2,820 \\
13,400 \\
12,800 \\
1,710\end{array}$ & $\begin{array}{l}1951 \\
1952 \\
1953\end{array}$ & $\begin{array}{lr}\text { Apr. } & 20 \\
\text { Mar. } & 25 \\
\text { Dec. } & 7\end{array}$ & $\begin{array}{l}14.9 \\
16.6 \\
20.6\end{array}$ & $\begin{array}{l}2,520 \\
3,320 \\
5,860\end{array}$ \\
\hline
\end{tabular}

(142) Conecuh River near Brooklyn, Ala.

Location.--Lat $31^{\circ} 10^{\prime}$, long $86^{\circ} 48^{\prime}$, in W $\frac{1}{2}$ sec. $6, T .2$ N., R. 13 E., on downstream side of right pler of bridge on U. S. H1ghway 29,3 miles downstream from Sepulga River and 7 miles southwest of Brooklyn.

Dra1nage area. $--2,460 \mathrm{sq} \mathrm{ml}$.

Records avallable.--U. S. Geological Survey: May 1935 to December 1953, da1ly gage helghts and discharges.

Gage.--Water-stage recorder. Datum of gage 1s $76.95 \mathrm{ft}$ above mean sea level, datum of 1929 supplementary adjustment of 1943. Prior to Sept. 5, 1937, wire-weight gage at same site and datum.

Stage-discharge relation.--Defined by current-meter measurements.

Historical data.--The flood of March 1929 reached a stage of about $47 \mathrm{ft}$, from information by Alabama State Highway Department.

Annual peak stages and discharges

\begin{tabular}{|c|c|c|c|c|c|c|c|}
\hline $\begin{array}{c}\text { Calendar } \\
\text { year }\end{array}$ & Date & $\begin{array}{c}\text { Gage } \\
\text { height } \\
\text { (feet) }\end{array}$ & $\begin{array}{l}\text { Discharge } \\
\text { (cfs) }\end{array}$ & $\begin{array}{c}\text { Calendar } \\
\text { year }\end{array}$ & Date & $\begin{array}{l}\text { Gage } \\
\text { height } \\
\text { (feet) }\end{array}$ & $\begin{array}{l}\text { Discharge } \\
\text { (cfs) }\end{array}$ \\
\hline 1929 & Mar. - & 47 & - & 1945 & Apr. 30 & 21.8 & 14,300 \\
\hline $\begin{array}{l}1936 \\
1937 \\
1938 \\
1939 \\
1940\end{array}$ & $\begin{array}{lr}\text { Jan. } & 22 \\
\text { Apr. } & 9 \\
\text { Mar. } & 20 \\
\text { Aug. } & 21 \\
\text { Feb. } 22\end{array}$ & $\begin{array}{l}34.4 \\
36.6 \\
38.0 \\
37.8 \\
28.0\end{array}$ & $\begin{array}{l}39,000 \\
50,100 \\
60,700 \\
59,100 \\
21,300\end{array}$ & $\begin{array}{l}1946 \\
1947 \\
1948 \\
1949 \\
1950\end{array}$ & $\begin{array}{lr}\text { May } & 25 \\
\text { Apr. } & 7 \\
\text { Dec. } & 1 \\
\text { May } & 5 \\
\text { Apr. } & 10\end{array}$ & $\begin{array}{l}31.4 \\
30.1 \\
38.6 \\
23.4 \\
18.8\end{array}$ & $\begin{array}{l}29,900 \\
27,000 \\
67,300 \\
16,400 \\
10,800\end{array}$ \\
\hline $\begin{array}{l}1941 \\
1942 \\
1943 \\
1944\end{array}$ & $\begin{array}{lr}\text { Dec. } & 29 \\
\text { Jan. } & 4 \\
\text { Mar. } 25 \\
\text { Mar. } 27\end{array}$ & $\begin{array}{l}23.3 \\
23.3 \\
37.8 \\
37.1\end{array}$ & $\begin{array}{l}14,400 \\
14,400 \\
59,100 \\
53,300\end{array}$ & $\begin{array}{l}1951 \\
1952 \\
1953\end{array}$ & $\begin{array}{ll}\text { Apr. } & 23 \\
\text { Mar. } 29 \\
\text { Dec. } 10\end{array}$ & $\begin{array}{l}23.7 \\
27.2 \\
30.7\end{array}$ & $\begin{array}{l}16,800 \\
21,900 \\
28,300\end{array}$ \\
\hline
\end{tabular}


(143) Murder Creek near Evergreen, Ala.

Location.--Lat $31^{\circ} 25^{\prime}$, long $87^{\circ} 00^{\prime}$, in $\mathrm{NW} \frac{1}{4} \mathrm{sec} .8, \mathrm{~T} .5 \mathrm{~N} ., \mathrm{R}$. $11 \mathrm{E}$, on left bank near upstream s1de of bridge on U. S. H1ghway 31, 1 mile upstream from Lou1svilie and Nashv1lle Ra1lroad. bridge and $2 \frac{1}{2}$ miles southwest of Evergreen.

Dra1nage area. $--170 \mathrm{sq} \mathrm{ml}$.

Records ava1lable.--U. S. Geological Survey: October 1937 to December 1953, dally gage helghts and discharges

Gage.--Water-stage recorder. Datum of gage is $178.29 \mathrm{ft}$ above mean sea level, datum of 1929 , supplementary adjustment of 1943 (levels by Corps of Engineers). Prior to Mar. 25, 1939, wire-welght gage at same site and datum.

Stage-discharge relation.--Defined by current-meter measurements below 10,000 cfs and extended above on basis of records for Sepulga River near McKenzie.

H1storlcal data.--The flood of March 1929 reached a stage of $26.6 \mathrm{ft}$, from 1nformation by local residents.

Annual peak stages and discharges

\begin{tabular}{|c|c|c|c|c|c|c|c|}
\hline $\begin{array}{c}\text { Calendar } \\
\text { year }\end{array}$ & Date & $\begin{array}{l}\text { Gage } \\
\text { height } \\
\text { (feet) }\end{array}$ & $\begin{array}{c}\text { Discharge } \\
\text { (cfs) }\end{array}$ & $\begin{array}{c}\text { Calendar } \\
\text { year }\end{array}$ & Date & $\begin{array}{l}\text { Gage } \\
\text { height } \\
\text { (feet) }\end{array}$ & $\begin{array}{l}\text { Discharge } \\
\text { (cfs) }\end{array}$ \\
\hline $\begin{array}{l}1929 \\
1938 \\
1939 \\
1940\end{array}$ & $\begin{array}{l}\text { Mar. }- \\
\text { Mar. } 16 \\
\text { Mar. } 30 \\
\text { Dec. } 17\end{array}$ & $\begin{array}{l}26.6 \\
16.6 \\
12.3 \\
11.0\end{array}$ & $\begin{array}{c}- \\
20,000 \\
5,610 \\
3,010\end{array}$ & $\begin{array}{l}1946 \\
1947 \\
1948 \\
1949 \\
1950\end{array}$ & $\begin{array}{lr}\text { Jan. } & 7 \\
\text { Apr. } & 2 \\
\text { Nov. } & 27 \\
\text { May } & 2 \\
\text { Apr. } & 5\end{array}$ & $\begin{array}{c}12.6 \\
11.0 \\
13.5 \\
- \\
8.9\end{array}$ & $\begin{array}{r}6,900 \\
3,180 \\
10,000 \\
a 3,500 \\
1,190\end{array}$ \\
\hline $\begin{array}{l}1941 \\
1942 \\
1943 \\
1944 \\
1945\end{array}$ & $\begin{array}{lr}\text { Dec. } & 24 \\
\text { Apr. } & 10 \\
\text { Nov. } & 8 \\
\text { Mar. } 29 \\
\text { Apr. } 29\end{array}$ & $\begin{array}{l}11.5 \\
10.2 \\
12.8 \\
12.6 \\
10.3\end{array}$ & $\begin{array}{l}3,900 \\
1,870 \\
6,800 \\
6,660 \\
2,090\end{array}$ & $\begin{array}{l}1951 \\
1952 \\
1953\end{array}$ & $\begin{array}{lr}\text { Mar. } & 29 \\
\text { Mar. } & 24 \\
\text { Dec. } & 4\end{array}$ & $\begin{array}{l}10.0 \\
10.2 \\
10.9\end{array}$ & $\begin{array}{l}2,220 \\
2,400 \\
2,840\end{array}$ \\
\hline
\end{tabular}

${ }^{a}$ No gage-he1ght record; peak discharge est1mated on basis of records for Escamb1a $\mathrm{R} 1 \mathrm{ver}$ at Flomaton and Sepulga River near McKenzie.

(144) Escambla Creek at Flomaton, Ala.

Location.--Lat $31^{\circ} \mathrm{O} 1^{\prime}$, long $87^{\circ} 15^{\prime}$, in NE $\frac{1}{4}$ sec. 33, T. I N., R. 8 E., on downstream side of left p1er of bridge on U. S. H1ghway' 31 at north edge of Flomaton, $1 \frac{1}{4}$ miles upstream from Loulsville and Nashville Railroad bridge, $1 \frac{1}{2}$ miles upstream from Alabama-Florida state line, and 4 miles upstream from mouth.

Dra1nage area. $--323 \mathrm{sq} \mathrm{m}$.

Records ava1lable.--U. S. Geological Survey: January 1939 to December 1951, da1ly gage heights and discharges. 1952, 1953, annual flood peaks. Prior to October 1949 publ1shed as

"Escambia River".

Gage.--Water-stage recorder. Datum of gage $1 \mathrm{~s} 52.40 \mathrm{ft}$ above mean sea level, datum of 1929. Prior to June 1, 1942, at site $400 \mathrm{ft}$ upstream at same datum. Since January 1952, crest-stage gage..

Stage-discharge relation.--Defined by current-meter measurements below 10,000 cfs and by slopearea determination at $41,400 \mathrm{cfs}$.

H1storical data.--The flood of March 1929 reached a stage of $25.9 \mathrm{ft}$, from information furn1shed by Alabama State H1ghway Department. Flood of Mar. 17, 1938, reached a stage of $18.0 \mathrm{ft}$, from floodmarks. 
Escamb1a River Basin

(144) Escambia Creek at Flomaton, Ala.--Continued

Annual peak stages and discharges

\begin{tabular}{|c|c|c|c|c|c|c|c|}
\hline $\begin{array}{c}\text { Calendar } \\
\text { year }\end{array}$ & Date & $\begin{array}{c}\text { Gage } \\
\text { height } \\
\text { (feet) }\end{array}$ & $\begin{array}{c}\text { Discharge } \\
\text { (cfs) }\end{array}$ & $\begin{array}{c}\text { Calendar } \\
\text { year }\end{array}$ & Date & $\begin{array}{c}\text { Gage } \\
\text { height } \\
\text { (feet) }\end{array}$ & $\begin{array}{l}\text { Discharge } \\
\text { (cfs) }\end{array}$ \\
\hline 1929 & Mar. - & 25.9 & - & 1946 & Mar. 29 & 10.6 & 7,060 \\
\hline $\begin{array}{l}1938 \\
1939 \\
1940\end{array}$ & $\begin{array}{lr}\text { Mar. } & 17 \\
\text { Sept. } & 27 \\
\text { May } & 1\end{array}$ & $\begin{array}{l}18.0 \\
19.3 \\
10.4\end{array}$ & $\begin{array}{r}33,000 \\
41,400 \\
6,250\end{array}$ & $\begin{array}{l}1947 \\
1948 \\
1949 \\
1950\end{array}$ & $\begin{array}{lr}\text { Mar. } & 8 \\
\text { Nov. } & 28 \\
\text { May } & 2 \\
\text { Apr. } & 5\end{array}$ & $\begin{array}{r}11.1 \\
14.5 \\
10.6 \\
8.9\end{array}$ & $\begin{array}{r}7,880 \\
15,400 \\
6,810 \\
4,570\end{array}$ \\
\hline $\begin{array}{l}1941 \\
1942 \\
1943 \\
1944 \\
1945\end{array}$ & $\begin{array}{lr}\text { Dec. } & 24 \\
\text { Jan. } & 2 \\
\text { Nov. } & 9 \\
\text { Mar. } & 23 \\
\text { Apr. } & 29\end{array}$ & $\begin{array}{r}8.0 \\
11.9 \\
10.8 \\
12.2 \\
9.6\end{array}$ & $\begin{array}{l}3,800 \\
8,250 \\
7,430 \\
9,990 \\
5,850\end{array}$ & $\begin{array}{l}1951 \\
1952 \\
1953\end{array}$ & $\begin{array}{lr}\text { Mar. } & 19 \\
\text { Mar. } & 24 \\
\text { Dec. } & 6\end{array}$ & $\begin{array}{r}10.3 \\
9.0 \\
11.8\end{array}$ & $\begin{array}{l}6,330 \\
4,670 \\
8,870\end{array}$ \\
\hline
\end{tabular}

(145) Escambia River near Century, Fla.

Location.--Lat $30^{\circ} 57^{\prime} 25^{\prime \prime}$, long $87^{\circ} 14^{\prime} 00^{\prime \prime}$, in sec. 10, T. $5 \mathrm{~N}$. , R. $30 \mathrm{~W}$, on left bank $16 \mathrm{ft}$ downstream from bridge on State Highway 4, 1.2 miles downstream from Escambla Creek, and $1 \frac{3}{4}$ miles east of Century.

Drainage area. $--3,817 \mathrm{sq} \mathrm{m1}$.

Records ava1lable.--U. S. Geological Survey: October 1934 to December 1953, da1ly gage he1ghts and discharges.

Gage.--Water-stage recorder. Datum of gage $1 \mathrm{~s} 28.34 \mathrm{ft}$ above mean sea level (State Road Department benchmark). Prior to Jan. 13, 1940, w1re-we1ght gage at same site and datum.

Stage-d1scharge relation.--Defined by current-meter measurements below $72,000 \mathrm{cfs}$.

Historical data.--Maximum stage known, $37.8 \mathrm{ft}$ in March 1929, from information by local residents (affected by fallure of a dam upstream).

Annual peak stages and discharges

\begin{tabular}{c|c|c|c||c|c|c|c}
\hline $\begin{array}{c}\text { Calendar } \\
\text { year }\end{array}$ & Date & $\begin{array}{c}\text { Gage } \\
\text { height } \\
\text { (feet) }\end{array}$ & $\begin{array}{c}\text { Discharge } \\
\text { (cfs) }\end{array}$ & $\begin{array}{c}\text { Calendar } \\
\text { year }\end{array}$ & Date & $\begin{array}{c}\text { Gage } \\
\text { height } \\
\text { (feet) }\end{array}$ & $\begin{array}{c}\text { Discharge } \\
\text { (cfs) }\end{array}$ \\
\hline 1929 & Mar. - & 37.8 & 315,000 & 1944 & Mar. 30 & 20.00 & 64,400 \\
1935 & Mar. 9 & 17.72 & 33,300 & 1945 & May 1 & 16.57 & 22,700 \\
1936 & Jan. 3 & 19.40 & 57,100 & 1946 & Mar. 29 & 17.69 & 33,900 \\
1937 & Apr. 11 & 20.05 & 64,400 & 1947 & Apr. 17 & 17.68 & 33,900 \\
1938 & Mar. 22 & 20.66 & 73,900 & 1948 & Dec. 2 & 20.43 & 70,200 \\
1939 & Aug. 22 & 20.10 & 69,800 & 1949 & May 3 & 17.13 & 27,700 \\
1940 & July 11 & 17.56 & 32,700 & & Apr. 6 & 15.38 & 17,400 \\
1941 & Mar. 10 & 13.11 & 11,000 & 1951 & Apr. 24 & 16.49 & 23,100 \\
1942 & Jan. 3 & 17.41 & 30,300 & 1952 & Mar. 26 & 17.25 & 28,800 \\
1943 & Mar. 26, 27 & 20.57 & 72,600 & & Dec. 7 & 18.59 & 45,300 \\
\hline
\end{tabular}




\section{Perd1do R1ver Basin}

(146) Perdido River at Barrineau Park, Fla.

Location.--Lat $30^{\circ} 41^{\prime} 25^{\prime \prime}$, long $87^{\circ} 26^{\prime} 25^{\prime \prime}$, in sec. 15, T. 2 N., R. 32 W., on right bank $25 \mathrm{ft}$ downstream from'h1ghway bridge, $1,000^{\prime} \mathrm{ft}$ downstream from Alilgator creek, and half a mile southwest of Barrineau Park.

Dra1nage area. $--394 \mathrm{sq} \mathrm{m}$.

Records ava1lable.--U. S. Geological Survey: June 1941 to December 1953, da1ly gage helghts and discharges.

Gage.--Water-stage recorder. Datum of gage is $25.71 \mathrm{ft}$ above mean sea level, datum of 1929. Prior to Aug. 22, 1949, staff gage at same site and datum.

Stage-discharge relation.--Defined by current-meter measurements below 5,500 cfs. Sight shifting at all stages.

H1storical data.--Maximum stage known, $25.7 \mathrm{ft}$ Mar. 15, 1929, from 1nformat1on by local residents.

Annual peak stages and discharges



${ }^{a}$ Result of discharge measurement. 